\title{
Hybrids of SNARE Transmembrane Domains and Aritificial Recognition Motifs as Membrane Fusion Inducing Model Peptides
}

\author{
Dissertation \\ for the award of the degree \\ “Doctor rerum naturalium” (Dr.rer.nat.) \\ of the Georg-August University Göttingen \\ Within the doctoral program chemistry \\ of the Georg-August University School of Science (GAUSS) \\ Submitted by \\ Jan-Dirk Wehland
}

From Verden/Aller

Göttingen, 2017 
Thesis Committee

\section{Prof. Dr. Ulf Diederichsen}

Institute of Organic and Biomolecular Chemistry, Georg-August University Göttingen

\section{Prof. Dr. Reinhard Jahn}

Max Planck Institute for Biophysical Chemistry, Göttingen

\section{Members of the Examination Board}

Reviewer: $\quad$ Prof. Dr. Ulf Diederichsen

Institute of Organic and Biomolecular Chemistry, Georg-August University Göttingen

Second Reviewer: Prof. Dr. Reinhard Jahn

Max Planck Institute for Biophysical Chemistry, Göttingen

Further members of the examination board:

\section{Prof. Dr. Kai Tittmann}

Department of Molecular Enzymology,

Georg-August University Göttingen

\section{Prof. Dr. Ivo Feussner}

Department of Plant Biochemistry,

Georg-August University Göttingen

\section{Dr. Shoubhik Das}

Institute of Organic and Biomolecular Chemistry, Georg-August University Göttingen

\section{Dr. Franziska Thomas}

Institute of Organic and Biomolecular Chemistry, Georg-August University Göttingen 

This work was supported by the Deutsche Forschungsgemeinschaft in the context of the collaborative research center 803 (SFB 803, Functionality controlled by organization in and between membranes). 

The work described in this doctoral thesis has been accomplished under the guidance and supervision of Prof. Dr. Ulf Diederichsen at the Institute of Organic and Biomolecular Chemistry (IOBC) of the Georg-August University Göttingen between August 2012 and October 2017.

I thank Prof. Dr. Ulf Diederichsen for the versatile research topic, his encouraging and friendly guidance as well as his practical and theoretical advice. Furthermore, I am grateful for the freedom of research and generous support during my PhD time. 

Für meine Mutter Elisabeth 



\section{Table of Contents}

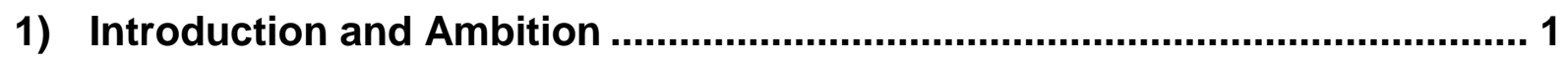

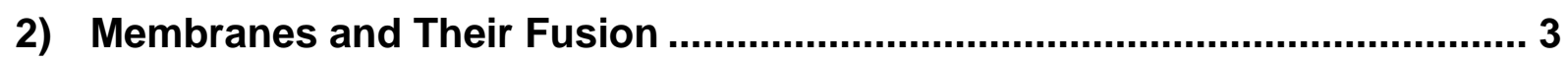

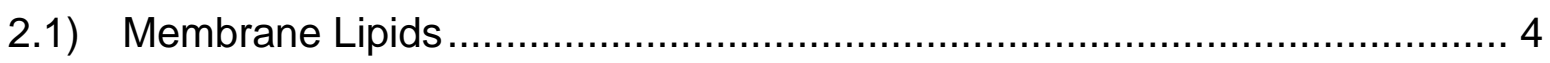

2.1.1) Effective Molecular Shape of Lipids ............................................... 6

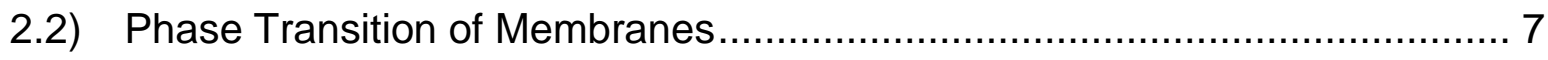

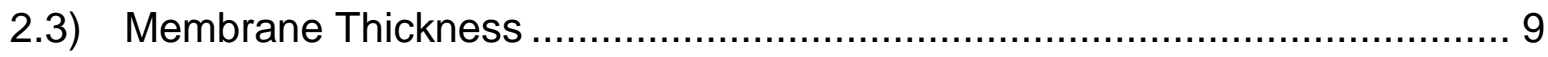

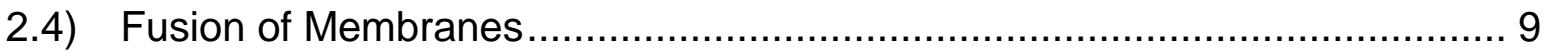

2.4.1) Influence of Lipids on Membrane Fusion .......................................... 12

2.4.2) Contribution of Proteins to Membrane Fusion.................................... 12

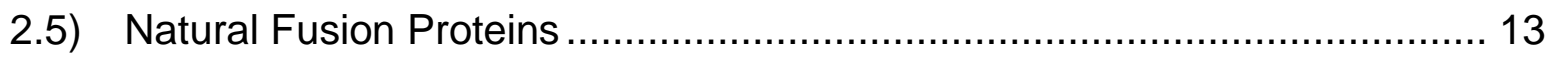

2.5.1) Viral Fusion Proteins .................................................................... 14

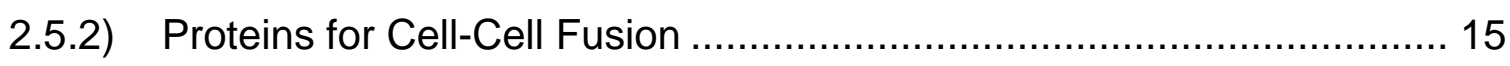

2.5.3) Endoplasmic Fusion Proteins: Atlastins and Mitosins ......................... 16

2.5.4) SNARE Protein Mediated Membrane Fusion...................................... 19

2.6) Model Systems for Membrane Fusion ..................................................... 22

2.6.1) Model Membranes …................................................................... 23

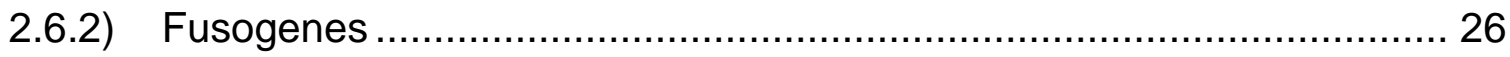

2.6.2.1) PNA-LR/TMD Peptide Hybrids as SNARE Mimetics ..................... 28

2.6.2.2) E3/K3-LR/TMD Peptide Hybrids as SNARE Mimetics.................... 30

2.6.3) Detection Methods for Membrane Characteristics ............................... 34

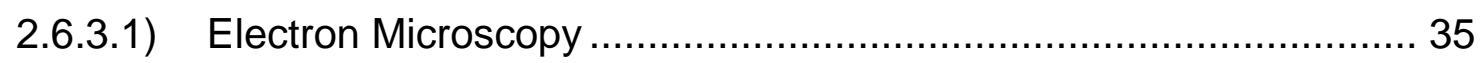

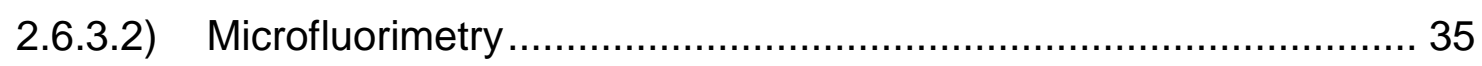

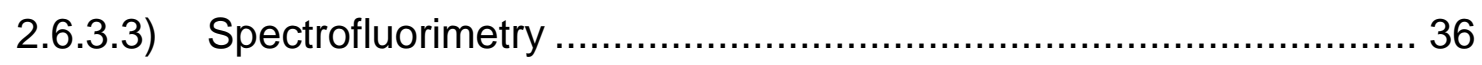

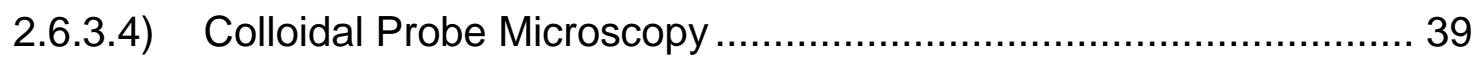

2.7) Applied Membrane Fusion System .......................................................... 41 
3.1) Influence of the Recognition Units of SNARE Mimetics on Leaflet Mixing ... 46

3.1.1) Recognition Orientation of PNA-LR/TMD Peptide Hybrids .................. 49

3.1.2) Recognition Orientation of E3/K3-LR/TMD Peptide Hybrids ................ 54

3.2) Influence of $C$-Terminal Carboxylic Acids and Amides of SNARE Mimetics on

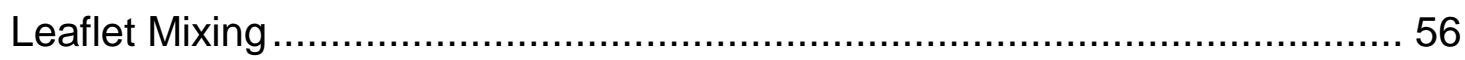

3.3) Significant Differences in Leaflet Mixing Due to $C$-Terminal Modifications .. 59

3.3.1) PNA-LR/TMD Peptide Hybrids with C-Terminal Carboxylic Acids ........ 59

3.3.2) PNA-LR/TMD Peptide Hybrids with C-Terminal Amides...................... 64

3.3.3) E3/K3-LR/TMD Peptide Hybrids with C-Terminal Carboxylic Acids ...... 68

3.3.4) E3/K3-LR/TMD Peptide Hybrids with C-Terminal Amides ................... 70

3.3.5) Conclusion of the Results Concerning C-Terminal Modifications.......... 72

3.4) Evaluation of Leaflet Mixing Assays .................................................... 73

3.4.1) Evaluation of Inner Leaflet Mixing Assays ........................................ 74

3.4.2) Evaluation of Liposome Stability ................................................... 76

3.4.3) Loss of Lipid Material During Proteoliposome Preparation ................... 84

3.4.4) Evaluation of the Insertion Degree of SNARE Mimetics in Model

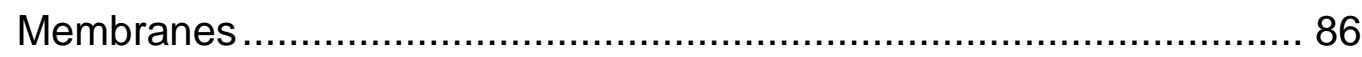

3.4.4.1) Evaluation by Density-Gradient Centrifugation ............................. 87

3.4.4.2) Evaluation by Fluorescent Probes .............................................. 89

3.4.5) Influence of Peptide Purity on Leaflet Mixing Experiments ................... 91

3.4.6) Size of Proteoliposomes and Its Influence on Fusion .......................... 93

3.4.6.1) Determination of Proteoliposome Size …………….................... 93

3.4.6.2) Influence of Proteoliposome Size on Membrane Fusion ................ 98

3.5) Results of Atomic Force Microscopy ……….................................... 100

3.5.1) Detection of Membrane Fusion by Colloidal Probe Microscopy .......... 100

3.5.2) Energy Barrier for $C$-Terminal Peptide Immersion into Membranes.... 103

3.6) Trigger for Peptide Mediated Membrane Fusion ................................... 106 


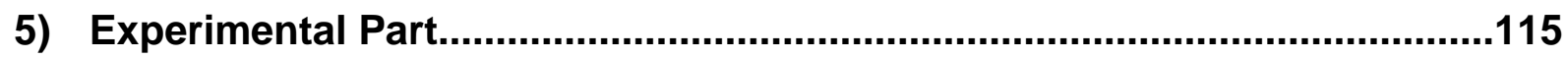

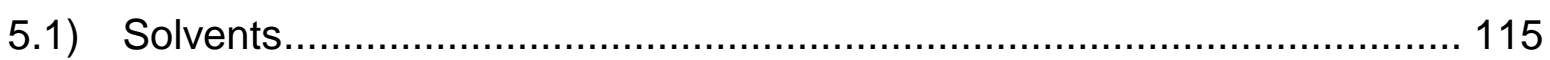

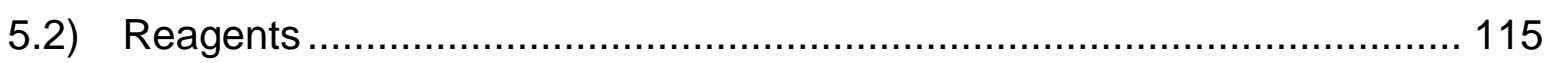

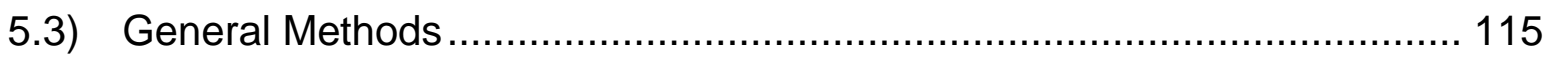

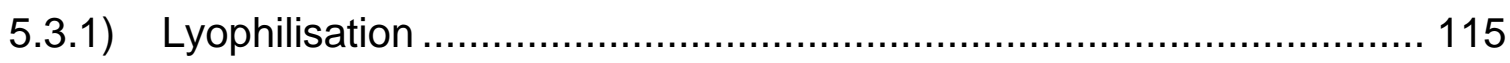

5.3.2) Reverse Phase Liquid Chromatography ........................................ 116

5.3.3) Size Exclusion Chromatography ……………............................... 116

5.4) Solid Phase Peptide Synthesis .......................................................... 116

5.4.1) Loading of the First Amino Acid ................................................. 116

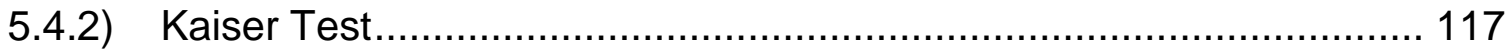

5.4.3) Estimation of the Loading Density............................................... 117

5.4.4) Synthesis of PNA-LR/TMD Peptide Hybrids ................................... 118

5.4.5) Synthesis of E3/K3-LR/TMD Peptide Hybrids ................................. 120

5.4.6) Acetylation of K3 and iE3 Peptides ............................................... 121

5.4.7) NBD Labelling of K3 Peptides...................................................... 121

5.4.8) Cleavage from the Solid Support ................................................. 121

5.5) Preparation of Model Membranes ……………................................... 122

5.5.1) Large Unilamellar Vesicles ......................................................... 122

5.5.2) Preparation of Pore Spanning Membranes and Proteoliposomes of

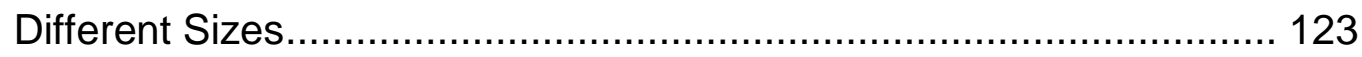

5.5.3) Preparation of Solid Supported Membranes and Colloidal Probe Supported Membranes ................................................................. 124

5.6) Modification of AFM tips and Peptide Attachment ................................. 124

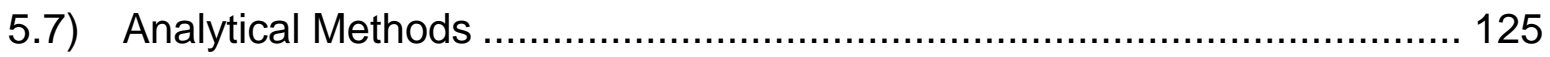

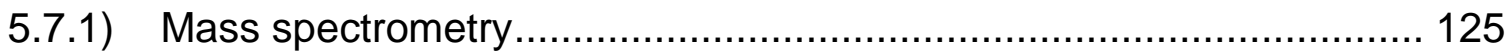

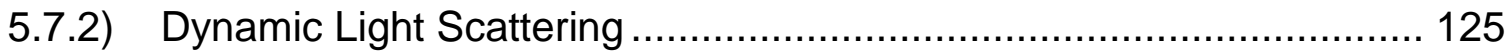

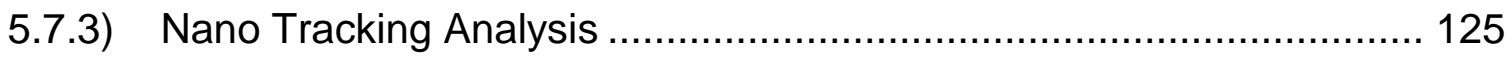




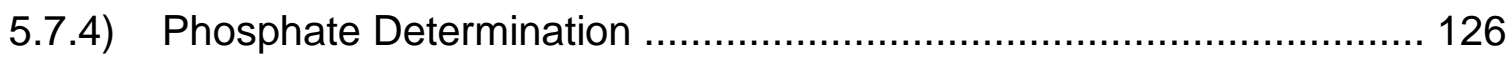

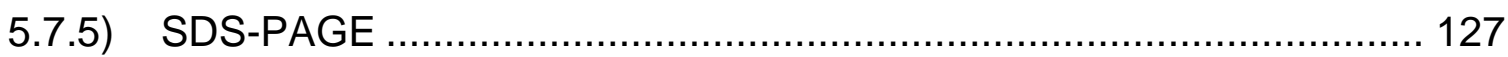

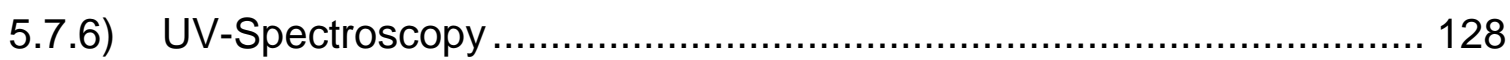

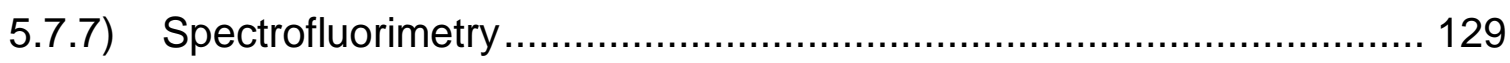

5.7.7.1) Total Leaflet Mixing (TLM) Assay .............................................. 129

5.7.7.2) Inner Leaflet Mixing (ILM) Assay ............................................. 130

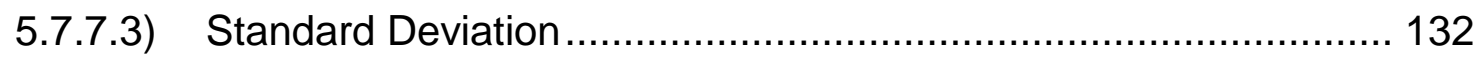

5.7.7.4) Trigger of Membrane Fusion by Soluble Peptidic Components.... 132

5.7.7.5) Determination of Lipid Loss Through Detection of the Fluorescence

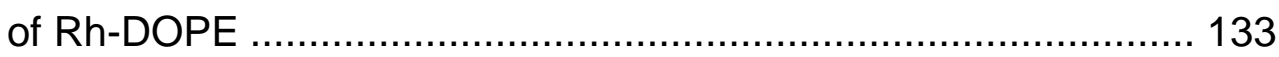

5.7.7.6) Proteoliposome Stability Test ................................................... 133

5.7.7.7) Peptide Insertion Test Based on Fluorescent Probes ................. 134

5.7.8) Peptide Insertion Test Based on Ultra-Centrifugation ......................... 134

5.8) Calculation of the Rate of Winding of $\alpha$-Helical Coiled Coils .................... 135

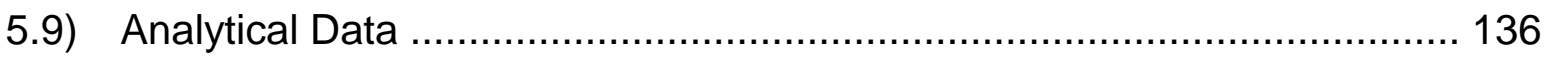

5.9.1) Transmembrane Domains ........................................................ 136

5.9.2) Acetylated iE3 and K3 Peptides................................................... 137

5.9.3) NBD Labelled K3 Peptide ......................................................... 138

5.9.4) PNA-LR/TMD Peptide Hybrids ...................................................... 139

5.9.4.1) PNA1/SybX(a) Peptides ......................................................... 139

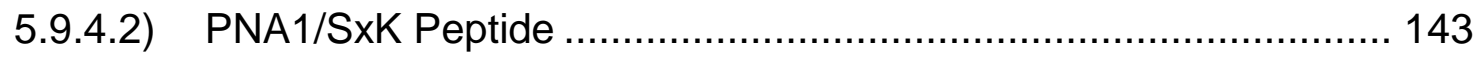

5.9.4.3) PNA2/SxY(a) Peptides .......................................................... 144

5.9.4.4) PNA3/SxY(a) Peptides ....................................................... 148

5.9.5) E3/K3-LR/TMD Peptide Hybrids ..................................................... 152

5.9.5.1) (i)E3/SybX(a) Peptides and Cys-E3/SybT ................................ 152

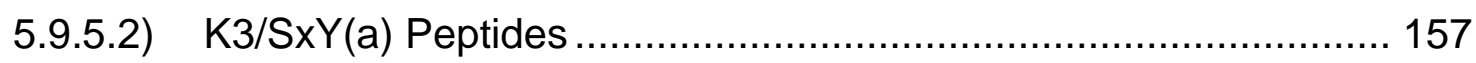

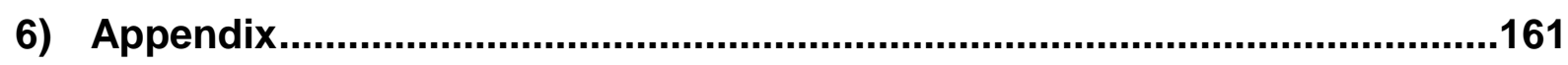




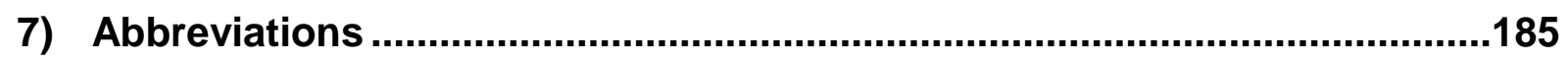

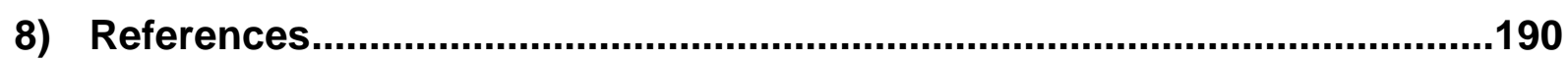




\section{1) Introduction and Ambition}

The merger of biological membranes is a crucial process of life. The fertilisation of an egg cell by a sperm and the subsequent development of tissues and organs of the embryo imply membrane fusion processes. The maintenance of physiology of the adult organism and the threat of vital functions by viruses are also dependent on the merger of membranes. ${ }^{[1-4]}$ Lipids represent the basis of membranes which serve as stable barriers between cells and compartments within the cell. They enable the establishment of different milieus which are of great importance for physiological functions. However, the controlled exchange of substances between cells and compartments are not less important. The communication between membrane separated spaces is enabled by membrane proteins. These proteins form ion channels or transfer signals through conformational changes from one side of the membrane to the other. Endo- and exocytosis are further processes for the exchange of substances. Vesicles loaded with substances merge with cell membranes and thereby release their cargo. ${ }^{[5]}$ Due to the fact that membranes are not prone to fuse spontaneously, proteins are necessary to mediate this process. Specialised proteins have been evolved and accompany life since its beginnings by mediating membrane fusion. One of this evolutionary conserved protein families are SNARE (soluble $N$-ethylmaleimide-sensitive factor (NSF) attachment protein receptor) proteins which are required for endocytic and secretory pathways in multiple organisms. ${ }^{[6]}$ The key actors of neuronal exocytosis of rattus norvegicus are the synaptic vesicle (SV) associated synaptobrevin 2 (Syb2), as well as syntaxin $1 \mathrm{~A}(\mathrm{~S} \times 1 \mathrm{~A})$ and the $25 \mathrm{kDa}$ synaptosome-associated protein (SNAP 25) which reside at the plasma membrane of the neuron. Syb2 and Sx1A own transmembrane domains (TMDs) connecting the proteins to their membranes. SNAP 25 is anchored at the membrane by palmitoylated cysteines in the linkage sequence of the two cytosolic domains. Syb2 and Sx1A have one cytosolic domain, respectively, which is linked to the TMD via a linker region (LR). These a-helical SNARE motifs form parallel intertwined four-membered coiled coils. However, it has also been demonstrated that SNARE motifs can congregate as antiparallel coiled coils. ${ }^{[3,7]}$ The formation of parallel four-helix bundles brings the SV membrane in close proximity to the plasma membrane of the synapse which is a basic requirement for membrane fu- 
sion. ${ }^{[8]}$ It is thought, that the recognition interaction starts $\mathrm{N}$-terminal and proceeds towards the $C$-termini including the LRs and TMDs of Syb2 and Sx1A. This is known as zippering hypothesis. ${ }^{[9,10]}$ Moreover, it has been supposed that during the recognition process resulting in membrane fusion, LRs and TMDs do not serve only as linkage and membrane anchors, but have also active roles. It is assumed, that the LRs/TMDs condense lipids which are suitable for the formation of fusion intermediates, deform membranes, perturb lipid packing, interact in a cooperative way during the last steps of fusion and relax curvature stress of the initial fusion pore. ${ }^{[11-14]}$

In the present study, SNARE mimetics were used to investigate the active role of the TMDs of Syb2 and Sx1A. Therefore, natural SNARE motifs were replaced by artificial recognition units. Either PNA oligomers or coiled-coil forming E3 and K3 peptides were linked to LRs/TMDs of Syb2 and Sx1A. ${ }^{[15,16]}$ PNA oligomers are well controllable concerning parallel or antiparallel recognition, but might not be able to induce zippering into the LRs/TMDs due to topological differences. However, the E3/K3 coiled coil system is comparable to native SNARE coiled coils and thus $C$-terminal zippering is conceivable. Both SNARE mimetics were modified concerning their $C$-terminal amino acids (aas). The natural aas threonine (Syb2) and glycine (Sx1A) were exchanged against glutamate and lysine as carboxylic acids or amides. These modifications change the $C$-terminal charge of the TMDs which are known to be crucial for fusion efficiency. ${ }^{[17,18]}$ The model peptides (PNA-LR/TMD and E3/K3-LR/TMD peptide hybrids) were used to investigate the influence of parallel and antiparallel recognition on the $C$-terminal modifications and to evaluate charge modifications concerning the last steps of fusion which are assumed to be mediated by cooperative interactions of the $C$-terminal parts of Syb2 and Sx1A. ${ }^{[13]}$ FRET based leaflet mixing assays with liposomes and pore-spanning membrane assays were used to determine the fusion efficiency of SNARE mimetics concerning membrane curvature. Furthermore, colloidal probe measurements were applied to examine single fusion events and to compare SNARE mimetics with the minimal fusion machinery of native Syb2, Sx1A and SNAP-25.[19] 


\section{2) Membranes and Their Fusion}

The basic structural motif of membranes is a bilayer consisting of two layers of lipids. The hydrophilic headgroups face the aqueous environment and the acyl chains forming a hydrophobic phase between the headgroups. ${ }^{[20]}$ As a key feature of life, lipid bilayers separate the interior of cells where vital processes occur from their environment and membranes are additionally present within the interior of cells enveloping multiple compartments which can be simplified seen as different reaction vessels for vital biochemical reactions. Beside the purpose of cell protection and compartmentation, membranes are involved in a variety of physiological functions like the controlled transport of substances (e.g. exo- and endocytosis), signal transduction, the production of energy and cell-cell adhesion as some examples. ${ }^{[21-23]}$ Therefore, membranes are not only structural, but also functional units. The enormous structural variety of membranes underlines the intriguing functional variety.

The fluid mosaic model of the structure of cell membranes by SINGER and NICOLSON was one of the first steps in the direction to evaluate biomembranes as functional units. They defined biomembranes as two-dimensional fluid structures containing peripheral and integral proteins laterally diffusing through the lipids and form functional complexes. ${ }^{[24]}$ However, this concept does not consider sufficiently the complex interactions between membrane components with cytosolic structures (e.g. cytoskeleton), extracellular receptor-ligand interactions and the interplay between membrane components like lipid-lipid, lipid-protein and protein-protein interactions. ${ }^{[22]}$ Moreover, lipid compositions of exoplasmic and cytoplasmic leaflets are not identical. Lipids and proteins are asymmetric distributed between the two leaflets, e.g. phosphatidylcholines (PC) and glycoproteins are mainly located in the exoplasmic leaflets. Whereas phosphatidylethanolamines (PE) can in principle be found in cytoplasmic leaflets. ${ }^{[23]}$ In addition to the transverse lipid distribution, lipids are also heterogeneously distributed in lateral dimensions. SIMONS and IKONEN described tightly packed membrane domains which are enriched in sphingolipids and cholesterol. They suggested that these lipid rafts diffuse with the otherwise fluid membrane and are involved in protein sorting processes. ${ }^{[25]}$ In conclusion, membranes are remarkable complex supramolecular structures and its functionality depends highly on the organisation of its components. 


\section{1) Membrane Lipids}

Membranes are built up by an astonishing number of different lipids. For instance, the membrane of erythrocytes consists of more than 100 lipids. ${ }^{[26]}$ The crucial feature of lipids is their amphiphilicity (polar headgroup and apolar acyl chains) which enables them to form membranes due to the hydrophobic effect. ${ }^{[27]}$ Membrane lipids consist mainly of phospholipids. An illustration of the common phospholipid structure is depicted in Figure 2.1-1. Frequently occurring polar headgroup residues are choline, ethanolamine, serine, glycerol or inositol. Latter one can also be phosphorylated. In addition to the variations of the headgroup regions one finds also many variations of the acyl chains. Typical acyl chains encompass 14 to 24 carbon atoms (e. g. myristate with 14 , palmitate with 16 or stearate residues with 18 carbon atoms). The chains are saturated or unsaturated (up to four double bonds). ${ }^{[28]}$ Furthermore, phosphoglycerolipids can carry different acyl chains (mixed acid lipids). [29]

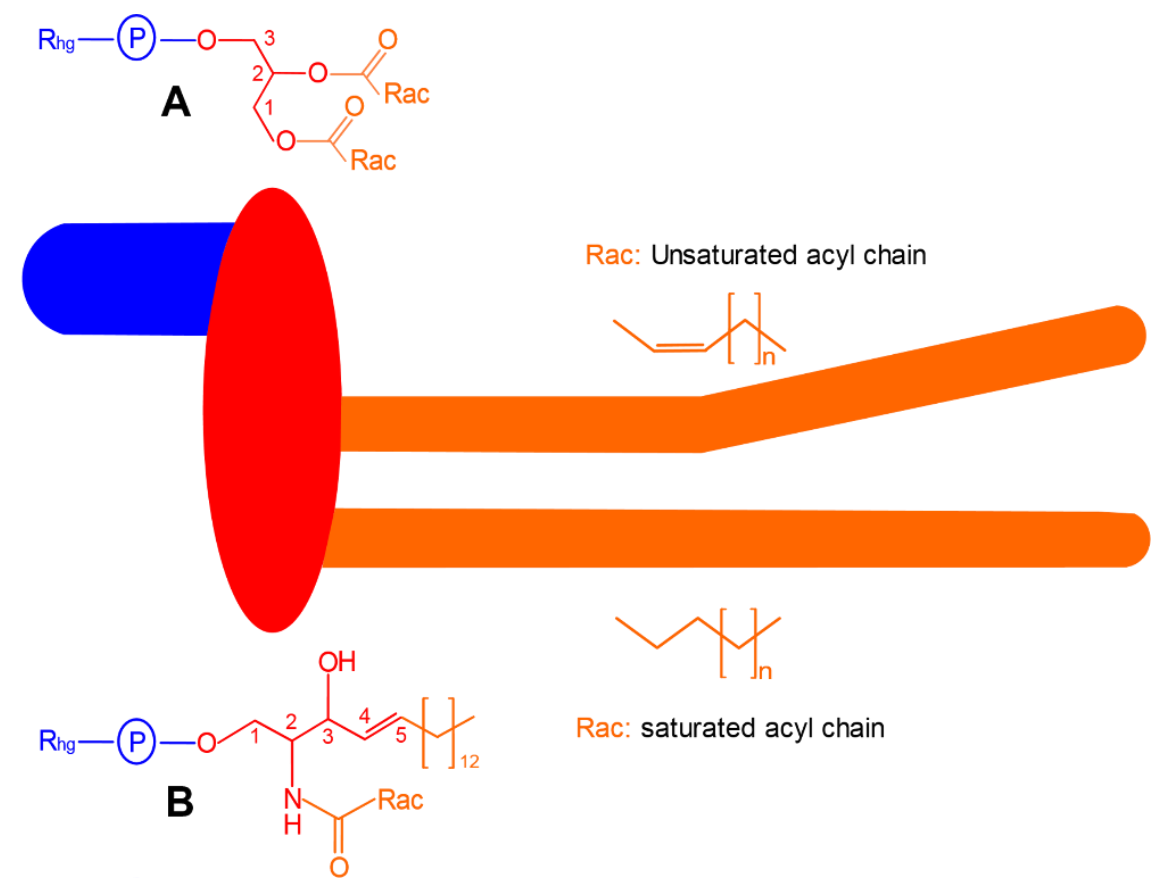

Figure 2.1-1 Illustration of the common structure of phospholipids with chemical structures of A) phosphoglycerolipids and B) sphingophospholipids (sphingomyelins): The phosphodiester (circled P, blue) is connected to the glycerol or sphingosine unit (red) and carry different headgroup residues ( $R_{h g}$, blue). In the case of phosphoglycerolipids, the glycerol unit is linked to two acyl chain residues ( $R_{a c}$, orange), which are saturated or unsaturated. For sphingophospholipids only one acyl chain is connected to the second carbon atom of the sphingosine unit via an amide bond (based on ref. 30). 
Beside phospholipids also other lipid groups exist which differ slightly in structure:

- Ceramides: The hydroxyl group in position one of the sphingosine unit is free.

- Phosphatidic acid: The phosphate group at the glycerine unit carry no further headgroup residues $(\mathrm{Rhg})$.

- Glycoglycerolipids and glycosphingolipids: The glycerol and sphingosine units are bound to sugar residues without phosphodiester bonds.

Another crucial group of lipidic components are sterols like cholesterol, ergosterol and fucosterol. ${ }^{[21]}$ Among sterols, cholesterol is the most common sterol in biological membranes with amounts of 4-40 mol-\% cholesterol in eukaryotic cell membranes. ${ }^{[28]}$ Synaptic vesicles (SVs) include 40 mol-\% cholesterol making them to one of the most cholesterol-rich organelles in nature. ${ }^{[31]}$ The hydroxyl groups of cholesterol form hydrogen bonds to the carbonyl carbons of the acyl chains of phosphoglycerolipids or to the hydroxy groups in position three of the sphingosine units in case of sphingolipids. ${ }^{[25]}$ The hydrophobic ring system is oriented towards the hydrophobic center of the bilayer (corresponds to the length of approximately 10 carbons of saturated hydrocarbon chains, Figure 2.1-2).[23]

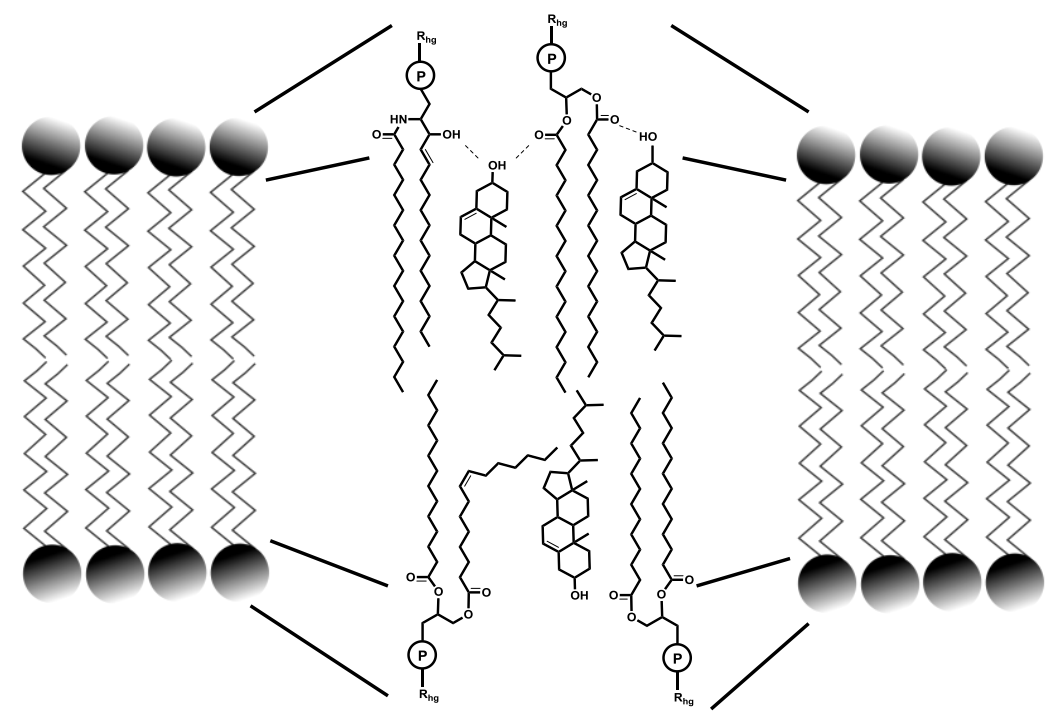

Figure 2.1-2 Illustration of the arrangement of cholesterol within a phospholipid bilayer: Dashed lines in the upper leaflet indicates hydrogen bonds between two cholesterols and one phosphoglycerolipid (right phospholipid). Furthermore, the hydrogen bond between one cholesterol and one phosphosphingolipid (left phospholipid) is depicted. In the bottom leaflet, cholesterol fills a gap in the hydrophobic interior of the membrane caused by a cis-double bond (based on ref. 25). 
In the fluid phase cholesterol induces higher amounts of trans-isomers of acyl chains and thus leads to thicker membranes. Moreover, cholesterol suppress the gel state and induces higher ordering of acyl chains in the fluid phase without significant loss of lateral mobility of lipids. ${ }^{[28]}$ Around one third of cholesterol in lipid compositions decreases permeability of phospholipid bilayers for small polar molecules and cholesterol reduces protein penetrations of the membrane. ${ }^{[32]}$ In conclusion, lipids constitute an enormous diverse group of substances and determine together with proteins the shape (e.g. thickness, curvature) and physical properties like permeability and thermotropic phase behaviour of membranes.

\subsection{1) Effective Molecular Shape of Lipids}

The molecular structure of single lipids mainly determines the structure of supramolecular structures above the critical micelle concentration (CMC).

A

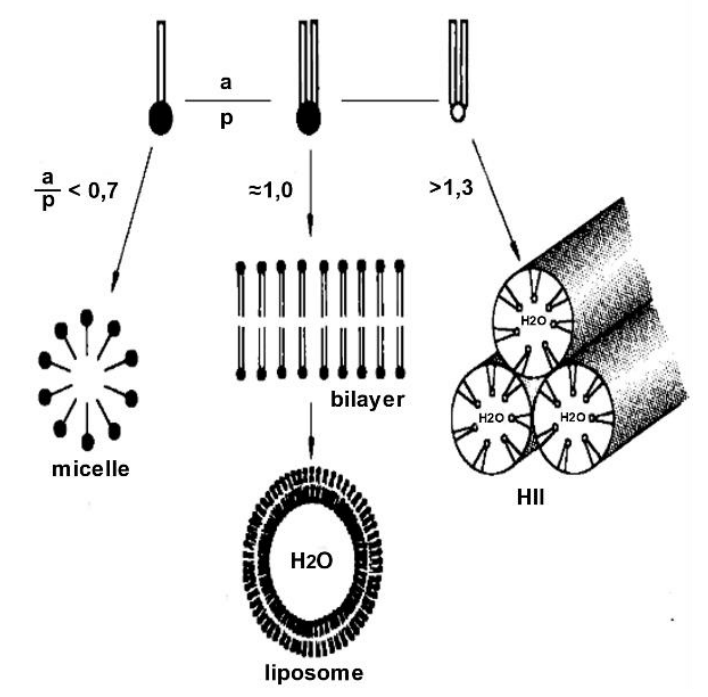

B

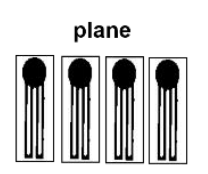

neg. curvature
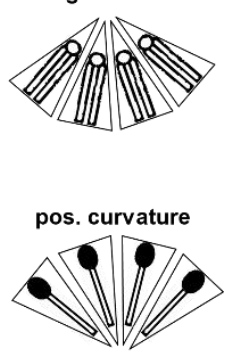

Figure 2.1.1-1 Effect of individual lipid shapes on the structure of lipid aggregates: A) Possible aggregate structures of lipids above the CMC in aqueous solutions depend on the ratio $(a / p)$ between the space requirements of apolar acyl chains (a) and the polar headgroups ( $p$ ) ( $H_{\|}$: hexagonal phase). B) Spontaneous curvature of lipid monolayers depends on the shape of single lipids (based on ref. 33 and 8). 
Like already mentioned, lipids consist of apolar acyl chains (a) and polar headgroups (p). The ratio of the demand of space of these two parts $(a / p)$ is a useful tool to predict the structure of supramolecular lipid aggregates (Figure 2.1.1-1, A). Lipids with an a/p ratio of approximately one (i.e. rod-like shaped lamellar lipids) form lamellar bilayers. Phosphatidylcholines like dioleoylphosphatidylcholine (DOPC) are an example for this group of lipids. If the ratio is smaller than 0.7 (i.e. inverted cone), lipids (e.g. lysophosphatidylcholine $(\mathrm{LPC}))$ form micelles. Lipids with a cone-like shape $(a / p=1.3)$ like phosphatidylethanolamines (e.g. dioleoylphosphatidylethanolamine (DOPE)) aggregate as inverted micelles (common designation: hexagonal phase $\mathrm{H}_{\|}$) with polar headgroups residing in the interior. ${ }^{[33,34]}$ However, lipids with a shape of a cone or inverted cone (non-lamellar lipids) participate also in lamellar structures. Thus, pure monolayers consisting of cone-like or inverted cone-like lipids exhibit so-called spontaneous curvatures, which are a crucial feature concerning the shape of membranes, especially during rearrangements like in the process of membrane fusion (Figure 2.1.1-1, B).

\section{2) Phase Transition of Membranes}

Like other substances, lipid bilayers occur depending on temperature as different phases with different physical properties. In the case of two-dimensional membranes crucial physical properties are:

- Area per lipid molecule and lateral diffusion of lipids.

- Lipid packing that refers to the average conformation of each carbon atom along the acyl chains.

- Tilt angle of lipids to the membrane normal.

- Heat uptake during phase transition (important for determination of phase transitions through differential scanning calorimetry). ${ }^{[21]}$

At low temperatures lipid bilayer are usually in the gel phase. At elevated temperatures bilayers change to the liquid phase $L_{\alpha}$ (thermotropic phase transition, Figure 2.2-1). In the gel phase, single lipids are tightly packed (all-trans conformation of acyl chains) and there is no lateral diffusion of the lipids at all. Above the main transition tempera- 
ture $\left(T_{\mathrm{m}}\right)$ contraction of acyl chains takes place and therefore the bilayer expands laterally and becomes approximately $15 \%$ thinner than in the gel phase. ${ }^{[23]}$ Due to reduced VAN-DER-WAALS interactions between acyl chains, the lateral mobility of lipids is increased. Typical diffusion coefficient of lipids in the liquid phase are in the range of $10^{-8}-10^{-7} \mathrm{~cm}^{2} / \mathrm{s} .{ }^{[21]} \mathrm{T}$ is influenced by polar headgroup structures (surface charge, state of protonation) and acyl chains (length, degree of saturation). Partly protonated headgroups form hydrogen bonds between the protonated and unprotonated states. This results in higher main transition temperatures. The same tendency is observed with increasing chain lengths and lower degrees of saturation. Trans-unsaturated chains results in higher $T_{\mathrm{m}}$ values than cis-unsaturated chains do. Therefore, cis-unsaturated acyl chains support the fluid state of membranes. The liquid phase $L_{\alpha}$ is a basic prerequisite for the physiological functionality of biomembranes. ${ }^{[28,33]}$

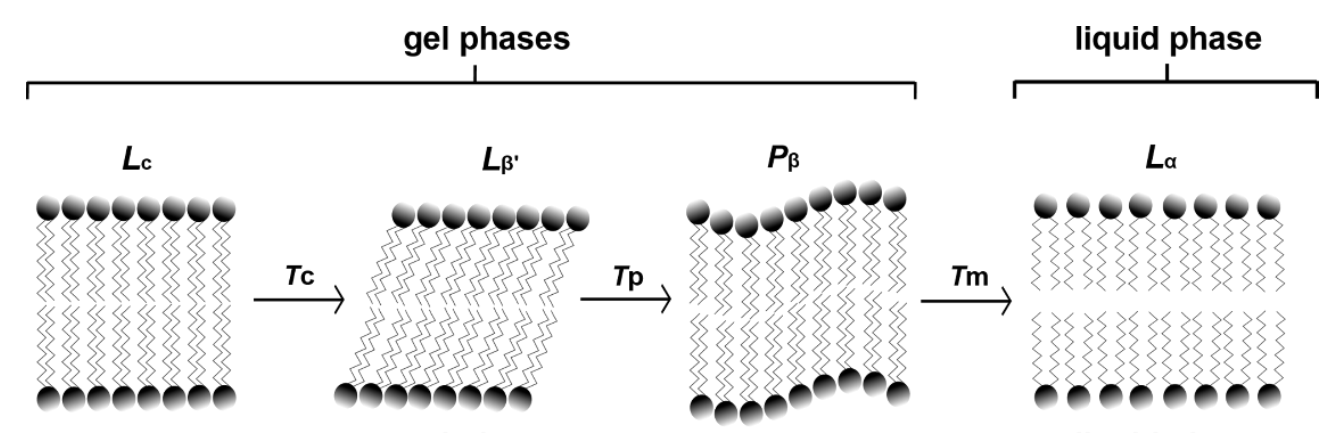

Figure 2.2-1 Illustration of thermotropic phase transitions of lipid bilayers. In each phase lipids differ concerning conformation, packing and lateral diffusion. Abbreviations: $L_{c}$ : sub-gel phase, $T_{\mathrm{c}}$ : sub transition temperature, $L_{\beta}:$ gel phase $\mathrm{II}, T_{\mathrm{p}}$ : pre-transition temperature, $P_{\beta}$ : ripple phase, $T_{\mathrm{m}}$ : main transition temperature and $L_{a}$ : liquid phase (based on ref. 28).

However, it is possible that two different phases coexist at the same temperature. This coexistence of lipid phases as nanoscopic domains is important for physiological functions like signalling, recruitment of proteins (preferred binding regions for more than 200 proteins) endo- and exocytosis.. ${ }^{[21,35]}$ Lipid domains consisting of sphingomyelins, glycosphingolipids, saturated phospholipids and cholesterol (or mixtures that include some of these lipids) exhibit resistance to detergent disruption and are of higher order than domains of other lipid mixtures. For instance, mixtures of glycosphingolipids and cholesterol have high packing densities due to interactions between headgroup carbohydrate residues of the sphingolipids and the hydroxyl groups of cholesterols. Furthermore, cholesterol acts as a stopgap for gaps emerged under the headgroup regions 
(unsaturated acyl chains or different lengths of acyl chains, Figure 2.1-1). ${ }^{[25]}$ These lipid domains are designated as lipid rafts, because a cluster of lipids diffuses through the fluid membrane environment. Lipid rafts in one monolayer might induce lipid rafts in the underlying monolayer, i.e. rafts span the whole membrane. ${ }^{[36]}$

\section{3) Membrane Thickness}

The thickness of membranes is an important feature concerning the insertion, orientation, activity and distribution of integral membrane proteins. For instance, due to a hydrophobic mismatch (thickness of membrane does not match the length of the hydrophobic region of an integral protein) proteins can arrange in clusters. ${ }^{[37]}$ Membrane thickness is extremely variable and reach from $3 \mathrm{~nm}$ to $10 \mathrm{~nm}$. One must distinguish between the thickness of the pure bilayer, the thickness of bilayers with hydration layers at the surfaces and the thickness of biomembranes with proteins. Pure PC membranes with two identical acyl chains of 14 carbon atoms (myristoleoyl residues) are $3.2 \mathrm{~nm}$ thick, by including the hydration layers a thickness of $4.7 \mathrm{~nm}$ was determined. Pure PC membranes with acyl chains of 22 carbon atoms and one double bond (erucoyl residues) have a thickness of $4.6 \mathrm{~nm}(6.1 \mathrm{~nm}$ with hydration layers). Obviously, one membrane thickness determinant is the length of the acyl chains. Cholesterol increases the thickness of PC membranes by $\sim 0.5 \mathrm{~nm}$. ${ }^{[38]}$ Typical biomembranes with various proteins have thicknesses of 7.5-10.0 nm. ${ }^{[39]}$ For instance, the erythrocyte membrane has a thickness of $8.5 \mathrm{~nm} .{ }^{[24]}$

\section{4) Fusion of Membranes}

The fusion process of pure lipid bilayers and biological membranes including proteins follow a comparable mechanism. ${ }^{40]}$ Therefore, first the mechanism of membrane fusion is described excluding the role of proteins. Afterwards, influences and contributions of proteins during the fusion process of biological membranes are elucidated. The initial step of the fusion process is a close intermembrane distance resulting in the so called hemifusion stalk (Figure 2.4-1, A-D). This structure is a widely accepted fusion 
intermediate firstly supposed by PALADE et al. in 1968, theoretically described by KOzLOV et al. in 1983 (later refined) and experimentally proved by YANG et al. in 2002 (X-ray diffraction). ${ }^{[41-44]}$ The stalk intermediate might expand to a hemifusion diaphragm, a structure with distal leaflets in hydrophobic contact (Figure 2.4-1, E). In the last step of fusion an aqueous pore (fusion pore) between the membrane encapsulated interiors is formed (Figure 2.4-1, F). It is uncertain, whether the fusion pore opening occurs directly from the hemifusion stalk (Figure 2.4-1, D) or whether the stalk expands to a hemifusion diaphragm and the fusion pore originates from this intermediate or both. ${ }^{[8]}$

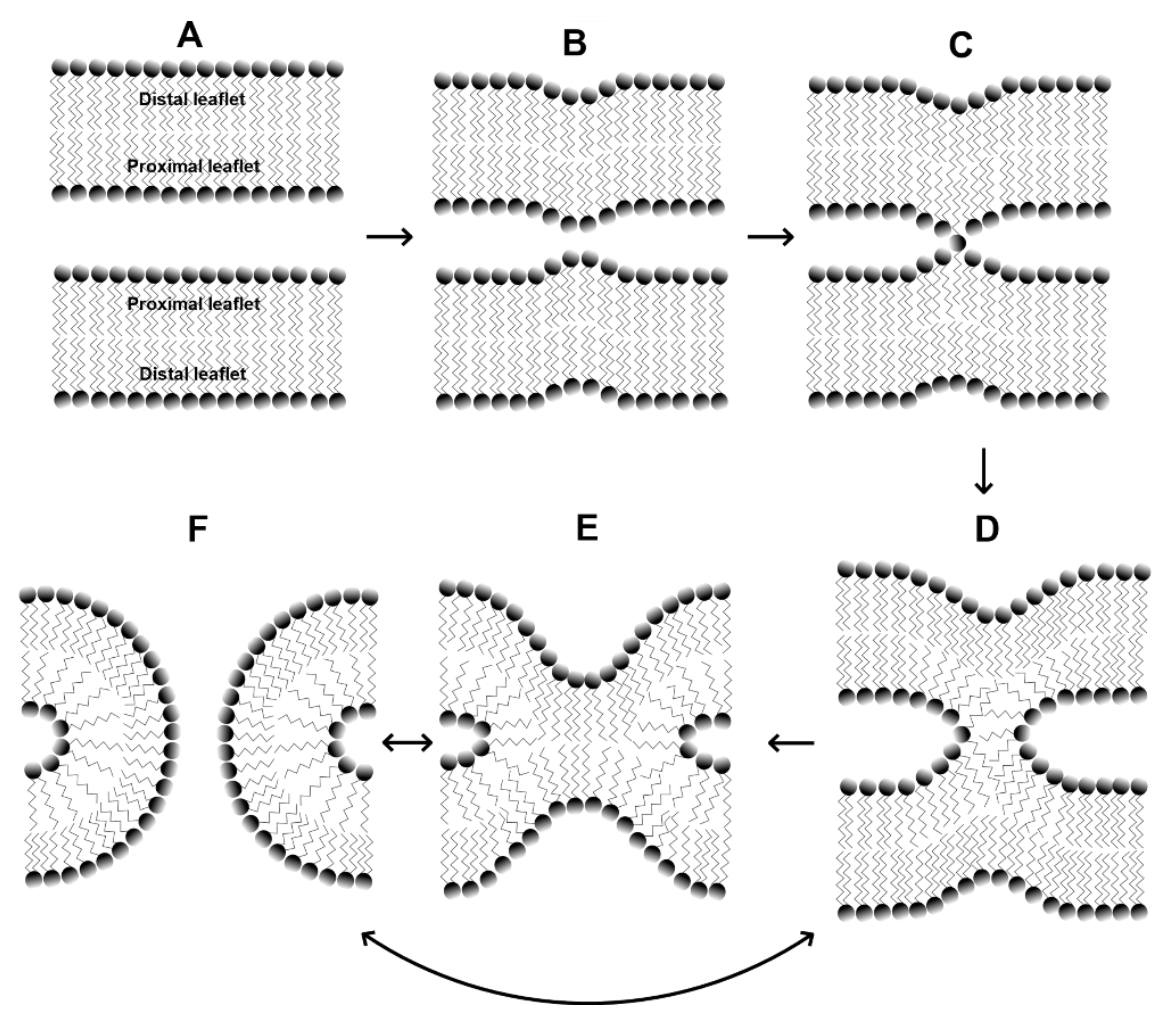

Figure 2.4-1: Supposed mechanism of membrane fusion: A) Apposed membranes separated by a water layer of 1-3 nm. B) Closer proximity between membranes, perhaps supported by point-like protrusions which minimise hydration forces. C) First hydrophobic contact through a splayed lipid. D) Extension of the hydrophobic contact area results in a hemifusion stalk. E) Possible extension of the stalk structure to a hemifusion diaphragm. F) Fusion pore evolves directly from stalk or transit diaphragm. Furthermore, the pore might close after formation and open again (pore flickering) (based on ref. 8 and 45).

Pure neutral lipid bilayers are separated by water layers of $1-3 \mathrm{~nm}$ (determined by gravimetric and osmotic stress methods). For instance, apposed DOPC and DOPE bilayers exhibit distances of $2.4 \mathrm{~nm}$ and $1.5 \mathrm{~nm}$, respectively. ${ }^{[46]}$ At a separating water 
layer of $0.9 \mathrm{~nm}$, the formation of the hemifusion stalk results in the relaxation of the hydration energy. ${ }^{[45]}$ At this close contact and degree of dehydration, it is possible, that one acyl chain of a single lipid is transferred from one membrane to the other. This constitutes the first hydrophobic contact between the membranes (Figure 2.4-1, C). Other lipids join this connection and proximal leaflets merge completely, while distal leaflets are still separated (hemifusion). ${ }^{[47]}$ To achieve close contact between membranes, it is necessary to dehydrate the aqueous interstice. It is suggested, that the energy for dehydration is the crucial barrier concerning the introduction of the fusion stalk. For pure DOPC membranes, the dehydration energy is $173 \pm 47 \mathrm{kB}_{\mathrm{B}} T$ and for an equimolar mixture of DOPC and DOPE it is $89 \pm 26 k_{\mathrm{B}} T$ (dehydration energies for areas of $40-50 \mathrm{~nm}^{2}$ at two flat apposed membranes). The energy of dehydrations depends on the area per lipid headgroup, which in turn depends on the headgroup residues and the degree of unsaturation of the acyl chain. Furthermore, the area per lipid is increased through lateral tension (e.g. in case of highly curved SUV membranes) or through the addition of cholesterol. For instance, the addition of $30 \mathrm{~mol}-\%$ cholesterol to DOPC membranes results in a dehydration energy of $\sim 105 \mathrm{kB} T$, which is much lower than for pure DOPC membranes (see above). During stalk formation, the thickness of the separating water layer increases above $1 \mathrm{~nm}$ indicating for favoured hydration conditions. Thus, rather the maximisation of hydration of the stalk intermediate is crucial for stalk formation than the minimisation of the intermembrane contact between the apposed membranes like it was proposed up to 2012. It was thought, that through a highly curved point-like protrusions of the apposed membranes, the contact zone is minimised and thus also the hydration repulsion between the membranes. ${ }^{[48]}$ Therefore, the calculated stalk energy of $40 \mathrm{kB}_{\mathrm{B}} T$ includes only bending and tilt deformations and excludes dehydration energy, which constitutes the main energy barrier for stalk formation. ${ }^{[44]}$ This current view on stalk formation is underlined by the observation, that the extent of stalk formation is not decreased with increasing acyl chain lengths and accompanied bending rigidity but exactly vice versa. ${ }^{[45]}$

The stalk intermediate expands and might create a contact zone between the distal leaflets. However, energetics for the diaphragm formation are high and therefore its formation is unrealistic. Therefore, it is suggested, that radial expansion of the stalk structure results in opening of the fusion pore. ${ }^{[49,50]}$ The initial fusion pore is not stable. There is an equilibrium between the hemifused membrane and an aqueous pore, 
which is designated as pore flickering. The fusion pore becomes stable by its expansion. ${ }^{[51,52]}$

\subsection{1) Influence of Lipids on Membrane Fusion}

It must be noted, that the effective molecular lipid shape and therefore the intrinsic curvature of lipids is beside dehydration energy another crucial factor for the stalk formation and the ongoing process of membrane fusion, because of the highly curved areas of the fusion intermediates. It has been demonstrated by GÜNTHER-AUSBORN et al., that the exogenous addition of lipids with a positive spontaneous curvature like LPC to the proximal leaflets inhibits stalk formation. This is explainable by the strongly negative curved areas of the proximal leaflets in the stalk structure. ${ }^{[53]}$ However, when LPC is specifically added to distal monolayers, the fusion pore formation is promoted, because the bending stress at the pore rims is reduced. On the other hand, lipids with a spontaneous negative curvature like arachidonic acid or DOPE promote fusion stalk formation when added to proximal leaflets, but inhibits/reduces fusion pore opening when added to distal leaflets. ${ }^{[54]}$ If cholesterol with an intrinsic negative curvature is present in both leaflets, it supports the permanent openness of the fusion pore by lowering curvature stress. ${ }^{[52,55]}$ In conclusion, specific lipids facilitate the formation of fusion intermediates due to their molecular shapes which are suitable for these intermediates. Furthermore, for membrane fusion crucial dehydration energies are affected by headgroup structures (Section 2.4).

\subsection{2) Contribution of Proteins to Membrane Fusion}

The surface of biological membranes is charged and densely mounted with integral and peripheral proteins, which are occasionally linked to sugar oligomers (glycoproteins). Such sugar residues are also found in lipidic structures (glycolipids). Thus, the actual lipid membrane is covered by a tight layer of biooligomers, which cause additional steric hindrance and repulsive forces compared to pure lipid bilayers. The distance between biomembranes approaching each other is usually $10-20 \mathrm{~nm} \cdot{ }^{[40,56]}$ 
Therefore, a protein-free contact zone must be established. Fusion proteins like SNAREs overcome this problem by pulling membranes in close proximity $(2-3 \mathrm{~nm})$ through complex formation and thereby displace sterically hindering proteins. ${ }^{[57]} \mathrm{An}-$ other strategy is the formation of $\mathrm{S} \times 1 \mathrm{~A}$ and $\mathrm{PI}(4,5) \mathrm{P}$ rich domains at the fusion sites which exclude hindering proteins. ${ }^{[58]}$ Sec/Munc (SM) proteins are thought to introduce lipid patches at the vicinity of Syb and Sx TMDs and membrane fusion might be facilitated at these patches. ${ }^{[59]}$ Additionally, integral proteins disturb the packing order of bilayers and transfer mechanical force on the membrane through conformational changes. ${ }^{[60]}$ Furthermore, integral proteins shape membranes, i.e. they induce negative or positive curvatures depending on the positions of sterically demanding aas like tryptophan within the proteins. ${ }^{[61]}$ Peripheral or slightly inserted membrane proteins also shape membranes against the intrinsic curvature which originate from the lipid composition. During the process of fusion, the cytoskeletal actin (in cooperation with other proteins) and dynamin GTPase support the extension of the fusion pore by maintaining membrane tension. ${ }^{[62,63]}$ Another possibilty making biomembranes prone to fuse are proteins like acyltransferases which catalyse lipid modifications and thereby establish a fusion-suitable lipid composition. Other proteins recruit suitable lipids. Flippases transfer lipids from one leaflet to the other and create asymmetric membranes which are necessary for the formation of fusion intermediates and finally for the fusion pore opening (Section 2.4.1). ${ }^{[21,40]}$

\section{5) Natural Fusion Proteins}

Natural fusion proteins are subdivided into two major groups: i) Exoplasmic fusion proteins act in the exterior of cells. For example, viral and cell-cell fusion proteins are involved in these types of membrane fusion. ii) Endoplasmic fusion proteins act in the interior of cells: atlastins (fusion of ER membranes), mitosins (fusion of mitochondrial membranes) and SNARE proteins (endocytic and secretory pathway fusion) are examples for these proteins. Within the following sections the different proteins are presented concerning their mechanisms of membrane fusion. 


\subsection{1) Viral Fusion Proteins}

There are four classes of viral fusion proteins which differ in their structural features and conformational changes during the process of fusion. However, all of them have intermediate hairpin structures in common. ${ }^{[2]}$ Below, the supposed mechanism of class 1 viral proteins is briefly presented by means of the well-known glycoprotein hemagglutinin $(\mathrm{HA})$ of the influenza virus: $\mathrm{HA}$ is a trimeric protein (each monomer include two disulfide-linked subunits: HA1 and HA2) and is anchored in the membrane of viral particles by $C$-terminal hydrophobic TMDs of the HA2 subunits. In the metastable prefusion state of HA, $N$-terminal fusion peptides (FP) of the HA2 subunits (kinked amphiphilic domains of $\sim 24$ aa) are shielded from the aqueous environment by the surrounding receptor-binding HA1 units. Latter units bind to sialic acid residues of the cellular membranes and evoke the uptake of the viral particles by endocytosis. In early endosomes, the HA proteins are exposed to low $\mathrm{pH}$ values $(\mathrm{pH}=5.3)$. Protonation induces conformational changes. Thereby, the FP is relieved and inserted into the endosomal membrane (Figure 2.5.1-1, A). Whereby, the first connection between both membranes is established. The FP causes defects in lipid packing of the target membrane and makes the membrane prone for early fusion stages. ${ }^{[64]}$ In the postfusion state a trimeric coiled coil (length of $13.5 \mathrm{~nm}$ ) has been formed and the TMDs and FPs are localised at adjacent positions within the fused membrane. ${ }^{[65]}$

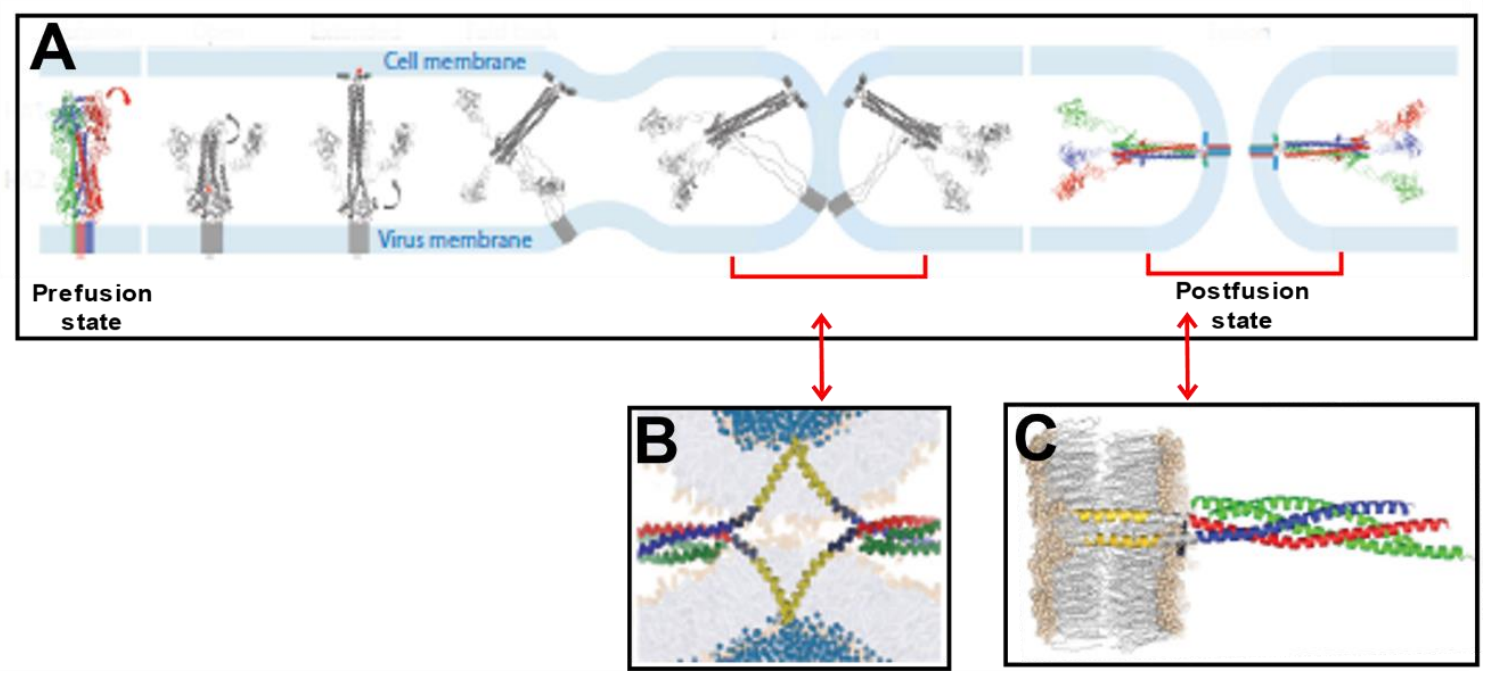

Figure 2.5.1-1 A) Supposed mechanism of HA mediated membrane fusion based on crystal structures of the metastable prefusion and stable postfusion state (coloured structures). Assumed intermediate structures of HA are depicted in grey. B) Two SNARE complexes with zippered SNARE motifs. C) Totally zippered SNARE cis-complex (based on ref. 10, 66 and 67). 
During this not fully elucidated process opposing membranes are pulled into close proximity allowing membranes to fuse via a hemifusion stalk (Figure 2.5.1-1, A). ${ }^{[4]}$ The proposed intermediate hairpin structure of the HA complex reminds one of the hairpin structure of the $N$-terminally zippered SNARE complex (Figure 2.5.1-1, A and B). Furthermore, the postfusion state of the HA complex resembles also the totally zippered SNARE cis-complex (Figure 2.5.1-1, A and C). This might be a hint that both mechanisms have similarities concerning structural and dynamic features and underlines the evolutionary relationship between the various types of fusion proteins. ${ }^{[40,66]}$

\subsection{2) Proteins for Cell-Cell Fusion}

Cell-cell fusion promoting proteins like syncytins (class I cell-cell fusion proteins) and epithelial fusion failure 1 protein (EFF-1 cell-cell fusion protein of class II) are the second important group of exoplasmic fusion proteins. They are involved in crucial processes like the formation of the placenta and the generation of the shape of organs including its tissues. Both classes of proteins share considerable structural similarities to the corresponding classes of viral fusion proteins. $10 \%$ of the human genome originates from retroviruses explaining the structural relationship between both groups of fusion proteins. ${ }^{[66]}$ However, in the case of viral infection, fusion proteins reside only in the viral membrane (heterotypic fusion), whereas cell-cell fusion proteins reside partly in membranes of both cells (homotypic fusion, Figure 2.5.2-1). ${ }^{[68]}$

A

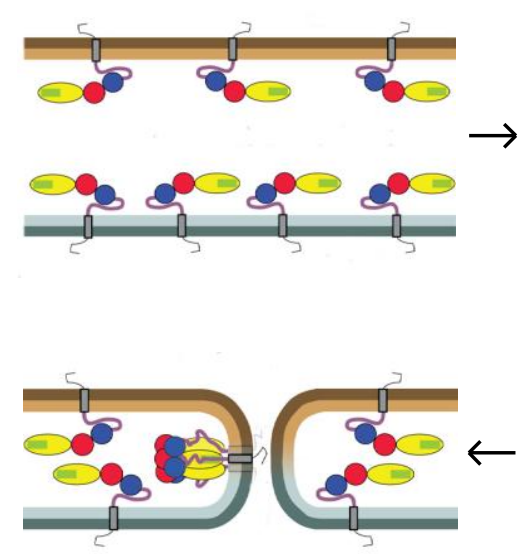

$\mathbf{F}$
B
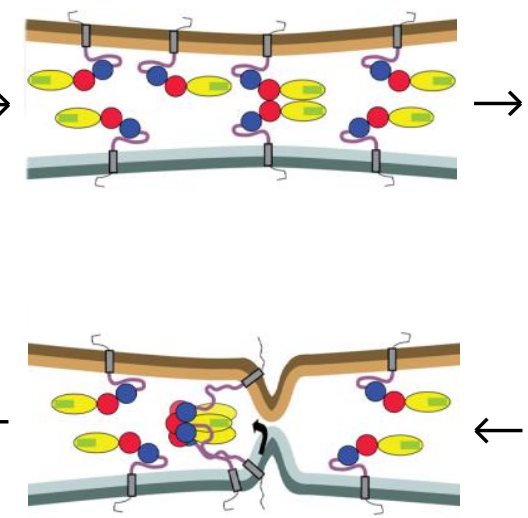

E
C

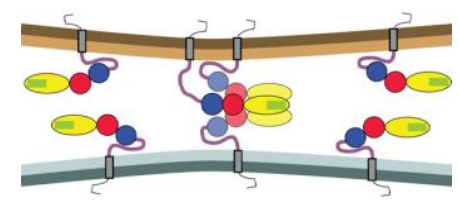

$\downarrow$

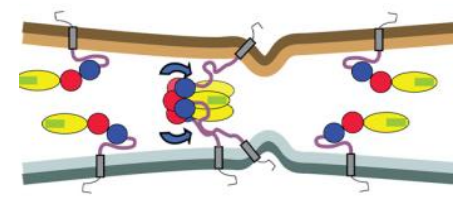

D

Figure 2.5.2-1 EFF-1 mediated membrane fusion: A) EFF-1 monomers cluster in the region of fusion. B) Dimerisation of monomers creates the first contact between the apposed membranes. C) An 
additional monomer binds to the already existing dimer. D) Domain III (DIII, blue circles) binds at the interface of domain I (DI, red circles) and domain II (DII, yellow ellipses), whereby cell membranes are pulled towards each other. E, F) $\mathrm{N}$-terminal parts of the TMDs (grey rods) interact with $\mathrm{N}$-terminal regions of DII. Afterwards, TMDs might zipper in a SNARE like fashion and thereby open the fusion pore (based on ref. 2).

Moreover, it is reasonable to assume that cell-cell fusion takes place directly after receptor binding without endocytosis. Class II proteins (EFF-1) have no FP and therefore another mechanism is expected which resembles more the SNARE mediated fusion process (Section 2.5.4):[2] It is supposed that after dimerisation of the extracellular domains, trimerisation of the dimers with single domains takes place moving the apposed membranes into spatial proximity (Figure 2.5.2-1, A-C). Conformational changes similar to viral fusion processes induce membrane curvature at the fusion site (Figure 2.5.2-1, D-E). ${ }^{[66]}$ The main difference between this mechanism and the SNARE mediated mechanism is the orientation of the cytosolic domains after fusion. During the supposed EFF-1-mediated mechanism, the recognising domains are directed into the centre of the fusion site where they might induce membrane curvature. Concerning the SNARE mediated fusion process, the domains are directed apart from the fusion site moving membranes into close proximity. The corresponding model for homotypic fusion in the case of EFF-1 suggests that after trimerisation of the $\mathrm{N}$-termini, the $\mathrm{C}$-terminal parts of the proteins bind on the surface of the $\mathrm{N}$-terminal parts pulling the membranes in closer contact. Moreover, the TMDs of the three proteins might interact with the tips of domain II and zipper towards their $C$-termini. Last conformational changes might be involved in fusion pore opening (Figure 2.5.2-1, E-F). ${ }^{[2]}$

\subsection{3) Endoplasmic Fusion Proteins: Atlastins and Mi- tosins}

Beside the SNARE proteins, atlastins and mitofusins are two other important endoplasmic fusion proteins. Both proteins are members of the dynamin superfamily of GTPases. Atlastins are involved in the structural organisation of the endoplasmic reticulum (ER). They are responsible for the maintenance of the ER architecture by pro- 
moting GTP dependent homotypic fusion between ER membranes. ${ }^{\left[{ }^{[6]}\right]}$ This is an important process beside fission. Mammalian atlastins are integral proteins with $\mathrm{N}$-terminal cytosolic GTPases domains $(G)$, juxtamembrane helical bundles (HB), two closely spaced TMDs and $C$-terminal amphiphilic tails (CT) which extend into the cytosol. Atlastins can form homodimers within the same ER membrane (cis-homodimers) or between two opposing ER membranes (trans-homodimers).
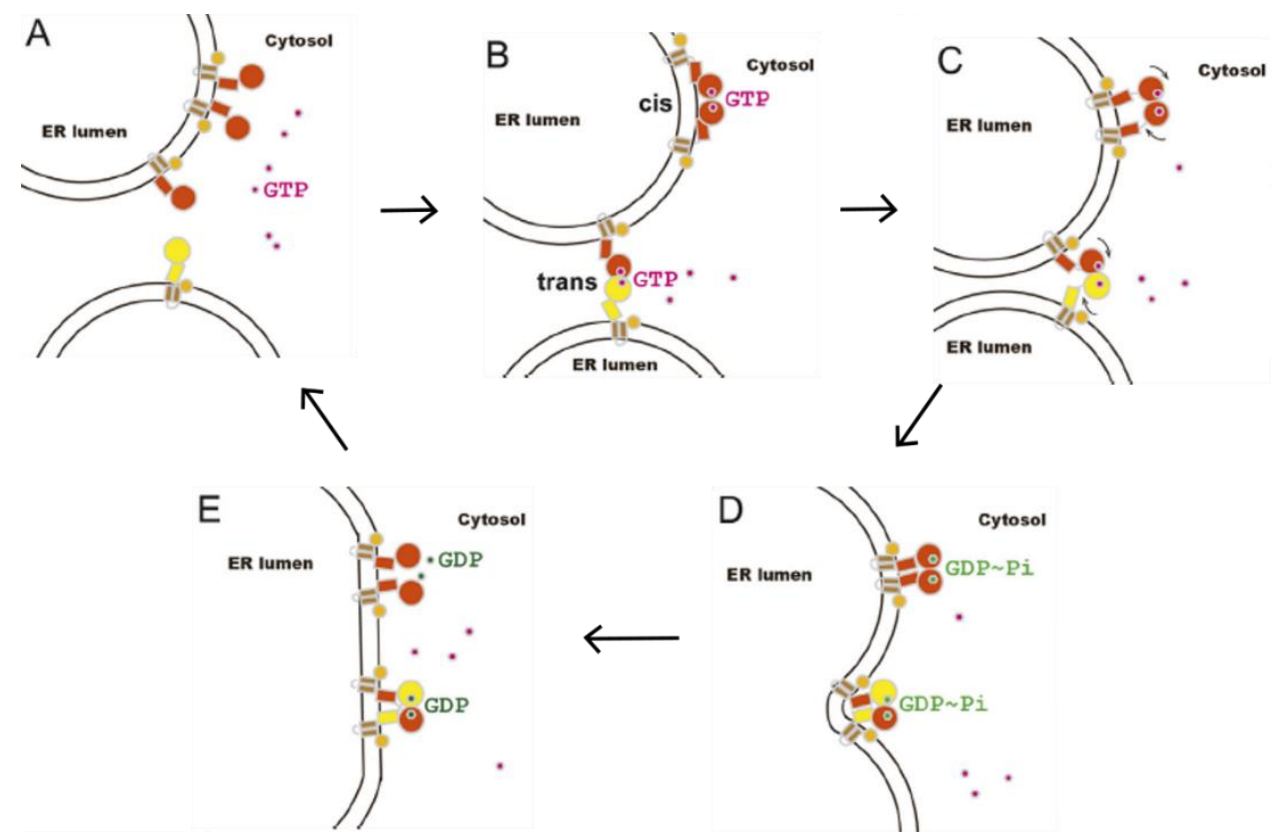

Figure 2.5.3-1 Illustration of the proposed fusion mechanism mediated by atlastins: A) Monomeric atlastins (brown and yellow) and free GTP (magenta). B) Dimerisation by binding GTP nucleotides within the same membrane (brown) and between apposed membranes (brown and yellow). C) G domains (big circles in brown and yellow) are disconnected from the HB domains (big rods in brown and yellow) resulting in conformational freedom (indicated by curved black arrows). D) Merged membranes probably induced by HB domain dimerisation and supported by membrane perturbing effects of TMDs (small brown rods) and CT (small orange circles). E) Dissociation of atlastin dimers (based on ref. 70).

GTP binds to the atlastin monomers and induces rapid dimer formation with the nucleotides residing in the interface of the $G$ domains (Figure 2.5.3-1, A-B). The dimers may adopt several conformations, but the exact relationship between membrane fusion and the sequence of conformational changes is only rarely understood. Dimers bound to GTP are relatively unstable, but hydrolysation of the nucleotides allows the association of the HB domains resulting in a stable dimer probably accompanied by merger of the membranes (Figure 2.5.3-1, C-D). Dissociation of the GDP bound or unbound state of 
the dimers releases atlastin monomers for further fusion cycles (Figure 2.5.3-1, E). The observation of cis-dimers is probably not only a consequence of fusion, because cis-interactions take also place before fusion. Cis-dimers might lower the energy barrier of fusion by destabilising membranes at the fusion site. The hairpin structure of the closely spaced TMDs in addition to perturbing effects of the amphiphilic CT might support the creation of fusion-prone membrane sites which collapse when HB domains form tight dimers. No other regulatory proteins seem to be involved in the atlastin mediated membrane fusion mechanism making it to a relative simple fusion system. However, SNAREs are found in the ER as well and might constitute another parallel fusion pathway which is eventually related to atlastin-mediated fusion. ${ }^{[70]}$

Like ER membranes mitochondrial membranes continually divide and fuse. The balance between these two processes play an important role for the structural and functional maintenance of mitochondria. Mitochondria possess outer and inner membranes and therefore it is reasonable that both membranes fuse in a coordinated fashion. Only in yeast, a potential candidate for coordination has been found, whereas a mammalian counterpart has not yet been identified. Nevertheless, it is known that sometimes only the outer membranes fuse, but the physiological relevance has not been explored. The human fusion protein of the inner membrane is the Opa1 (Optic atrophy defect) protein and mitofusins (Mfn1 and Mfn2) are the fusion proteins of the outer membrane. ${ }^{[71,72]}$ Within the $\mathrm{N}$-terminal region human mitofusins have a cytosolic GTPase domain followed by coiled-coil forming domains which are separated by two closely spaced TMDs. The $C$-terminal coiled-coil forming domains of mitofusins residing in apposed membranes associate into an antiparallel coiled-coil complex tethering the membranes with a gap of at least $9.5 \mathrm{~nm}$ (length of antiparallel coiled-coil complex). ${ }^{[73]}$ At this distance, fusion of membranes is unlikely without further interactions bringing the membranes in closer contact. The GTPases domain might be involved in this step, because it is known that nucleotide binding and hydrolysis induce trans-domain dimerisation and conformational changes of this domain, respectively. Both processes might lead to closer membrane contact. ${ }^{[74]}$ However, an exact structural mechanism linking tethering action and conformational changes of mitofusins with membrane merger is currently missing. 


\subsection{4) SNARE Protein Mediated Membrane Fusion}

Membrane fusion events of the secretory pathway are mediated by SNARE proteins. The key elements for protein mediated neuronal exocytosis are Sx1A, SNAP 25 and Syb2 and constitute the minimal fusion machinery, i.e. the reconstitution of these proteins in model membranes is sufficient to observe fusion in vitro. ${ }^{19]}$ Sx1A and SNAP 25 reside in the plasma membrane of neuronal synapses and form a binary prefusion acceptor complex (SNAP 25 contributes two $\alpha$-helices). Syb2 resides in the membrane of the synaptic vesicle (SV). Through complexation of the membrane distal $N$-terminal halves of Syb2 and the acceptor complex a partial zippered four-helix bundle (SNARE complex zippers up to the -1-layer) is formed which docks liposomes to the plasma membrane. ${ }^{[5]}$ Further complexation into the second halves of the SNARE motifs leads to closer intermembrane contact (zipper hypothesis). ${ }^{[9]}$ However, the formation of the cytosolic trans-SNARE complex approaches opposing membranes only to distances of 2-3 nm (LRs and TMDs are not associated). ${ }^{[57,76]}$ For the establishment of a lipidic connection as starting point of the actual fusion process the intermembrane distance must be smaller than $1 \mathrm{~nm}$ (Section 2.4). ${ }^{[45]}$ Therefore, the zippering process progresses into the LRs and TMDs of Syb2 and Sx1A accompanied by local perturbations of the membranes at the fusion site through the exerted mechanical force. ${ }^{[10,14]}$ The velocity of leaflet mixing of reconstitution experiments $(8.0 \mathrm{~ms})$ is far away from physiological neurotransmitter release after electrical stimulation $(<0.5 \mathrm{~ms})$ and it is suggested that other proteins than the above mentioned accelerate the merger of membranes. ${ }^{[57,77]}$ Moreover, the process of native membrane fusion is temporal and spatial controlled. Thus, control mechanisms for fusion priming, targeted trigger and fast release of neurotransmitter are present in natural systems.

The starting point of the SNARE mediated fusion process might be the binding of the cytosolic Sec/Munc (SM) protein Munc18-1 to the closed conformation of Sx1A ( $N$-terminal $\mathrm{H}_{\mathrm{abc}}$ domain interacts with the SNARE motif of $\left.\mathrm{S} \times 1 \mathrm{~A}\right)$ and inhibits the formation of the Sx1A/SNAP 25 acceptor complex (Figure 2.5.4-1, A). Sx1A forms clusters with acidic lipids like $\mathrm{PIP}_{2}$ or $\mathrm{PIP}_{3}$ under the contribution of calcium ions. Thereby, proteins enrich at the fusion site (spatial control). ${ }^{[58,78]}$ Munc13, another cytosolic SM protein, mediates the transition of the closed conformation of $\mathrm{S} \times 1 \mathrm{~A}$ to the open conformation. 
At that point, SNAP 25 might bind to Sx1A forming the acceptor complex (Figure 2.5.4-1, B). Afterwards, both SM proteins support the formation of the partial zippered SNARE complex under contribution of Syb2. ${ }^{[79]}$ For instance, it is known that Munc18-1 interacts with the membrane proximal region of Syb2 and thus supports the SNARE complex assembly. Cytosolic complexin (Cpx) binds to the Sx1A/SNAP 25 complex (or partial zippered SNARE complex). ${ }^{[80,81]}$ The $\mathrm{N}$-terminal accessory helix might serve as a placeholder for the $C$-terminal part of Syb2 and inhibit $C$-terminal complexation when Syb2 binds. ${ }^{[81]}$ Consequently, it has a clamping function and holds the tethered SV in a fusion-ready (primed) state. The connection between the SV and the plasma membrane is also supported by the calcium sensor synaptotagmin (Syt) which resides in the SV membrane and binds to the plasma membrane (Figure 2.5.4-1, C). Upon influx of calcium (temporal control), the C2 domains of Syt interact stronger with negatively charged lipids of the plasma membrane and induce membrane curvature as well as closer intermembrane contact. ${ }^{[82,83]}$ Furthermore, Cpx is released by the calcium influx (Figure 2.5.4-1, D). Now, zippering proceeds into the LRs and TMDs of Syb2 and Sx1A. Mechanical force is exerted on the membranes which induces the lipidic fusion process. ${ }^{[84]}$ Zippering into the $C$-terminal regions of Syb2 and $S \times 1 A$ is accompanied by TMD motions pulling the membranes together. Thereby, the $C$-termini of both proteins remain initially attached to the membrane surfaces. When the tilting forces become too strong, $C$-termini detach from polar headgroups and migrate into the hydrophobic core of the membranes causing a rearrangement of lipids. As a consequence of lipid rearrangement the fusion pore opens. ${ }^{[14]}$ Initial fusion pores flicker rapidly $(4000 \mathrm{~Hz})$ between opened and closed stage before permanently close or open. ${ }^{[52]}$ Stabilisation of the open pore is achieved by its expansion. Membrane tension might be a crucial factor for pore extension and it is known that dynamin in cooperation with cytoskeletal components induces such tension. ${ }^{[62,63]}$ Recently, it was suggested that the fusion pore is neither lipidic nor proteinous but is mainly lined by lipids and to some degree by the $C$-termini of Syb2 and Sx1 A (Figure 2.5.4-1, E). ${ }^{[85]}$ 


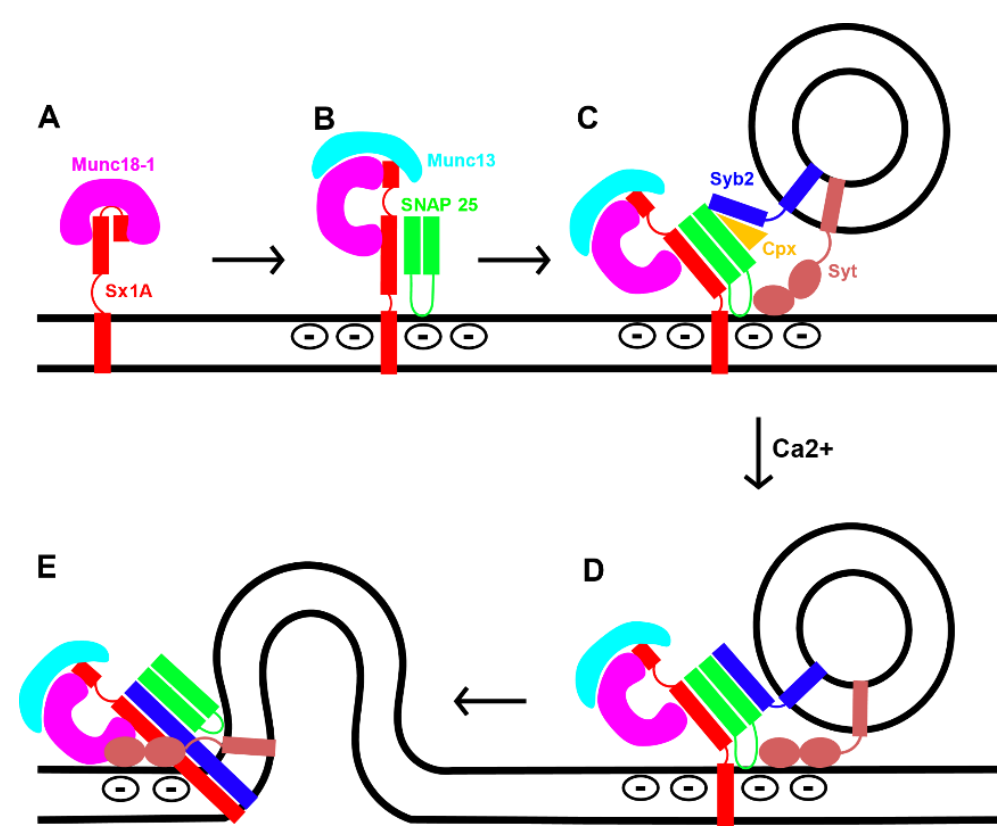

Figure 2.5.4-1 Supposed fusion mechanism mediated by the key SNARE proteins Syb2, Sx1A and SNAP 25. Regulatory proteins Munc18-1, Munc13, Cpx and Syt are involved in spatial and temporal control of the fusion process beside other proteins (not shown) (based on ref. 79 and 84).

In conclusion, seven proteins crucial for neuronal exocytosis were presented. The represented interplay of these proteins during SNARE mediated fusion is partly speculative, because several - sometimes contradictory - interactions for one protein are described in literature. Thus, one protein might act at different stages of the fusion process or in a combined synergetic manner with other proteins. It is obvious that the process is highly complex. Moreover, it is conceivable that there is not the one and only mechanism of fusion but several mechanisms might lead to membrane merger. 


\section{6) Model Systems for Membrane Fusion}

Due to the importance of biological membranes for life and their complexity concerning organisation and function, model systems were introduced. The overall goal of model systems is the reduction of complexity and thus the facilitation for scientists to assess the individual roles of membrane components. Through model membranes, it is possible to investigate physical properties like phase behaviour, lateral mobility and flip-flop mobility (asymmetric membranes) of lipids. Furthermore, membranes can be examined concerning their dynamics like protein clustering within the membrane and protein recognition between membranes as a crucial factor for membrane fusion. ${ }^{[86]}$ Fusion is defined as merging of two distinct membranes into one continuous membrane accompanied by the leakage-free formation of aqueous connections (fusion pores) between membrane encapsulated interiors. This directed process is mediated by fusogenes, i.e. substances which interact with membranes to fuse them. The exchange of lipids between merged membranes, the exchange of contents between the membrane encapsulated interiors and the alteration in membrane size (in regard to liposomes) are crucial features of membrane fusion which are exploited for its detection. ${ }^{[56]}$ However, leaflet mixing is also conceivable without fusion in the form described in Section 2.4, e.g. through the diffusion of lipids. ${ }^{[87]}$ Leakage during the fusion process can falsify the results of content mixing assays and the detection of increased particle sizes by indirect physical methods (e.g. DLS) after fusion is not necessarily the result of membrane fusion, because liposome aggregation lead to size increase as well. [56] In order to evaluate a study concerning membrane fusion, it is very important to understand how the fusion process is observed and how the observation is interpreted. For the detection of membrane features and fusion processes many methods like nuclear magnetic resonance (NMR) spectroscopy, electron paramagnetic resonance (EPR), mass spectrometry (e.g. NanoSIMS), differential calorimetry scanning (DSC), electron and fluorescence microscopy as experimental approaches are available. Additionally, computer simulations like full-atomistic and coarse-grained models serve as theoretical approaches. [21,86]

The combination of model membrane, fusogene and detection method is the actual model system which is used to elaborate membrane related topics. Within the following sections the three pillares for studying membrane fusion are presented. 


\subsection{1) Model Membranes}

Over the last decades several model membranes have been developed. They differ concerning the arrangement of the membrane. Liposomes are spherical particles which diffuse in bulk suspension or are immobilised through tethering at solid supports. ${ }^{[86]}$ Other model membrane systems are presented by free-standing black lipid or planar supported membranes which correspond more to the planar geometry of plasma membranes than to native vesicles. A combination of the latter bilayer systems constitute pore spanning membranes. ${ }^{[88]}$ In this section, four model membranes are presented concerning their structure, preparation and application (Figure 2.6.1-1, A-D).

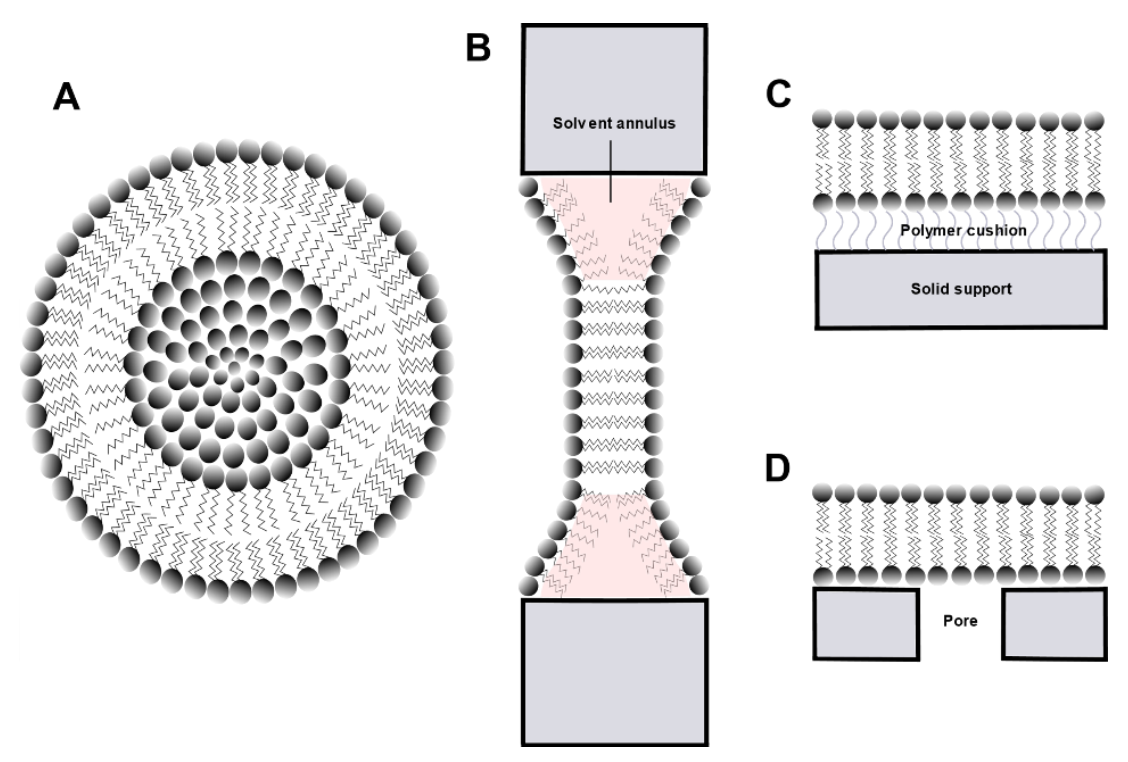

Figure 2.6.1-1 Selection of model membranes: A) Cross section of a liposome, B) black lipid membrane, C) planar supported membrane (freely supported) and D) pore-spanning membrane (based on ref. 21 and 88).

Liposomes: In 1966 PAPADADJOPOULOS and BANGHAM observed that temperature and divalent cations like calcium ions influence the permeability for univalent ions (e.g. sodium) of liquid crystals composed of phosphatidylserine (PS) and phosphatidylcholine (PC) lipids, respectively. Liquid crystals were prepared by sonication of aqueous lipid dispersions and it is mentioned that the crystals were able to capture ions. ${ }^{\left[{ }^{[9]}\right.}$ Although it is not directly mentioned, this was one of the first studies using liposome-like structures as model system for biological membranes. Depending on the preparation technique different structured and sized liposomes can be created: Multilamellar vesicles 
(MLVs) are onion shaped liposomes with several bilayers formed by hydration of lipid films stuck to glass walls. The films are prepared by evaporation of organic solvents containing the dissolved lipids. The hydration time influences the liposome size and the amount of entrapped aqueous content. MLV formation requires mechanical agitation of the hydrated films. ${ }^{[32,90]}$ Small unilamellar vesicles (SUVs) with a diameter of 30 to $50 \mathrm{~nm}$ are mainly prepared by ultrasound sonication of MLVs dispersions, whereas liposome size homogeneity depends on time of treatment and used lipid composition. Another preparation technique is the French press. ${ }^{[91]}$ The strong curvature of SUVs may lead to asymmetric membranes with an appropriate mixture of lipids. Furthermore, SUVs are relatively unstable due to bending stress which leads to spontaneous fusion events. ${ }^{[21,32]}$ Large unilamellar vesicles (LUVs) have mean diameters between 100 and $400 \mathrm{~nm}$ and are usually formed by extrusion techniques. ${ }^{[92,93]}$ Giant unilamellar vesicles (GUVs) with diameters of $15 \mu \mathrm{m}$ or more are obtained by electroformation. ${ }^{\text {[94] In }}$ general, liposome preparations are rapidly performed without much effort. Liposomes are mostly stable structures which can be stored at least over several days. However, oxidation processes lead to scission of the acyl chains of lipids at double bonds. Hydrolysis processes cleave acyl chains at the ester boundary. Such chemical modifications lead to changes of bilayer permeability, phase transition temperature and liposome size. Therefore, protective measures must be considered, like degassed water for buffer solutions, avoidance of strong irradiation, usage of inert gases and low temperatures for lipid/liposome storage. Further, the avoidance of strong acidic or basic $\mathrm{pH}$ values in liposome suspensions is important. ${ }^{[90]}$ Examples for the application of liposomes are the examination of membrane fusion with reconstituted native SNARE proteins or the transfer of encapsulated cargos (e.g. DNA) to living cells as a potential drug delivery system. ${ }^{[19,95]}$

Black lipid membranes (BLMs, Figure 2.6.1-1, B) are prepared by spreading lipids dissolved in an organic solvent over a small hole (e.g. $\sim 1 \mathrm{~mm}$ in diameter) in the separating wall of a container filled with an aqueous solution. The lipids form a bilayer which spans the hole. ${ }^{[96]}$ BLMs are fragile structures which tend to rupture and the reconstitution of native proteins might be problematic because of organic solvents which are

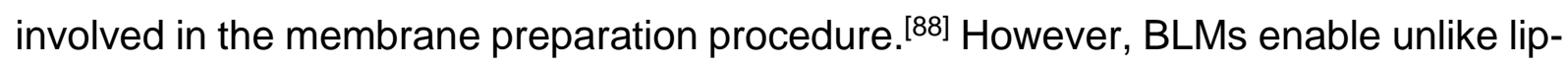
osomes easy access to both sides of the bilayer. Single ion channels can be examined 
by conductance measurements. Furthermore, fusion events and effects of proteins adsorbed to the membrane surface can be investigated using fluorescence microscopy techniques. ${ }^{[97]}$

Planar supported membranes (Figure 2.6.1-1, C) are fixed lipid bilayers (covalently or through ion bridges) via the inner leaflet to substrates like silicon, glass or gold and are named integrated bilayers. The fixation of the inner leaflet restricts the lateral mobility of lipids and reconstituted proteins, whereby bigger molecules are stronger restricted. The introduction of a super-thin water layer $(10 \AA)$ between substrate and bilayer creates freely supported bilayer which allows lipids and proteins to move laterally. The introduction of a polymer cushion into the interspace of membrane and substrate increases additionally the distance between substrate and membrane and facilitates lateral mobility. ${ }^{[21,98]}$ Bilayers are deposited by liposome spreading on pure quartz slides or on lipid monolayers which are transferred to slides from the water-air interface of a LANGMUIR-BLODGETT trough. If liposomes with integrated proteins (proteoliposomes) are used, protein containing supported membranes are created. Moreover, the preparation method for planar lipid bilayers using liposomes and preformed monolayers allows the generation of asymmetric membranes. ${ }^{[99]}$ Planar supported bilayers are suitable for the determination of fusion intermediates and kinetics of single fusion events by fluorescence microscopy. ${ }^{[77]}$

Pore-spanning membranes (Figure 2.6.1-1, D): A combination of BLMs and planar supported membranes are pore-spanning membranes. Silicon-nitride grids are covered with layers of titanium and gold. Latter is treated with an amphiphilic thiol making the surface prone for vesicle spreading. GUVs are spread on the porous surface creating a planar membrane which covers pores of $5 \mu \mathrm{m}$ in diameter and rims of the grid. ${ }^{[100]}$ Pore-spanning membranes combine the advantages of BLM (accessibility of both membrane sides) and planar supported membranes (high stability, avoidance of organic solvents during preparation). By laser scanning confocal microscopy it is possible to observe single fusion events and to distinguish between docking, hemifusion and full fusion during the fusion event. ${ }^{[88]}$ 


\subsection{2) Fusogenes}

In the 1970's calcium ions were used to investigate membrane fusion of SUVs. It was suggested that calcium ions interact with the polar headgroups of PS and reduce repulsive forces between membranes. Thus, liposomes can approach each other. The formation of a nearly water-free trans-complexes by polar headgroups of the apposed membranes and the calcium ions reduces the inter-membrane spacing further (Figure 2.6.2-1, A). Moreover, calcium ions induce phase transition of the outer leaflets (fluid $\rightarrow$ crystalline) resulting in an unstable state making membranes susceptible to

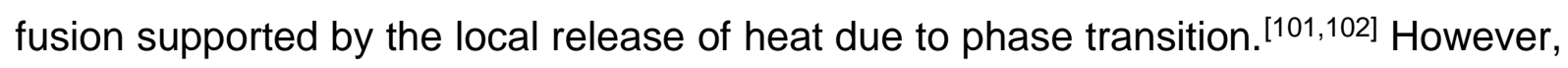
the product of fusion was not necessarily a bigger intact liposome. Other lipidic structures like flattened bilayer dices were observed. ${ }^{[101]}$ Additionally, liposomes can just be deformed or ruptured by calcium ions and membrane interactions are nonspecific. Consequently, the calcium-liposome system is confined concerning the investigation of natural membrane fusion processes. ${ }^{[103]}$ Therefore, other fusogenes were created which mimic the ability of integral fusion proteins to fuse membranes.
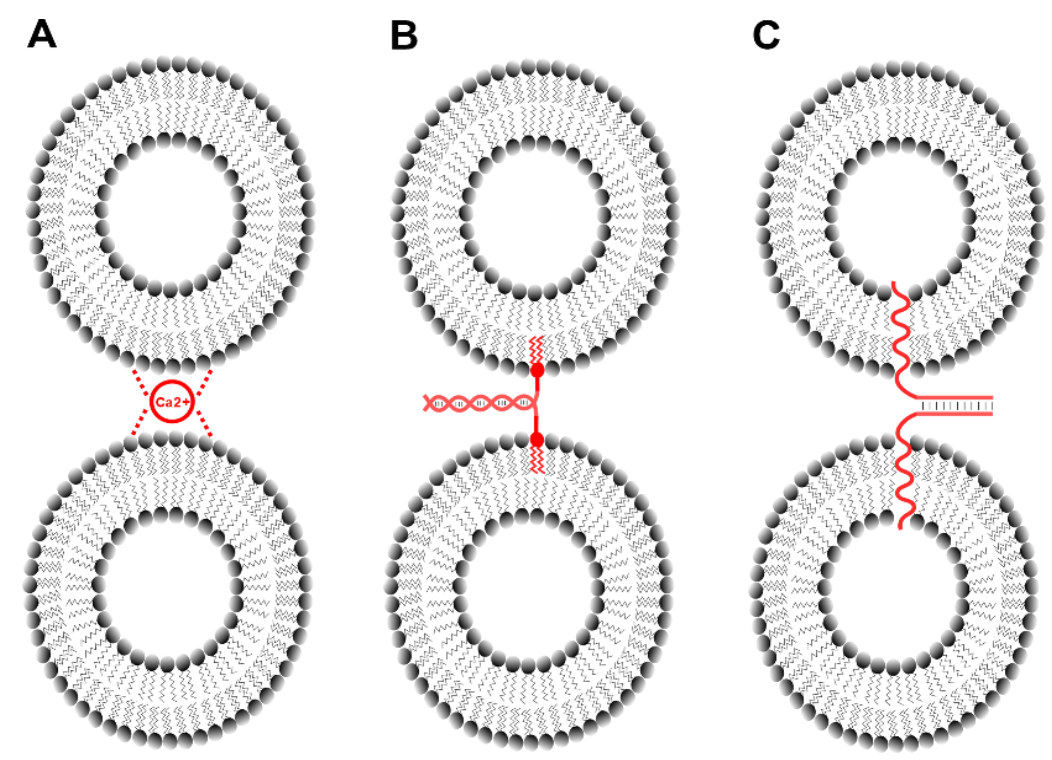

Figure 2.6.2-1 Illustration of docked liposomes mediated by different fusogenes (red): A) Calcium ions form a tethering complex with the lipid headgroups. B) Headgroups of lipids are linked to DNA recognition motifs. C) Integral membrane proteins are linked to recognition motifs like PNA or coiled-coil forming peptides (based on ref. 56). 
For instance, a construct consisting of a lipid anchor and a DNA based recognition motif was synthesised to mimic the minimal fusion machinery of SNARE proteins (Figure 2.6.2-1, B). Dioctadecyl-glycerol anchored complementary DNA sequences of 24 nucleobases or partially hybridised DNA sequences of $27 \mathrm{bp}$ anchored by two cholesterol anchors (12 membrane proximal base pairs to ensure double cholesterol anchoring and 15 free nucleobases) fuse SUVs and LUVs. By the induction of non-complementary DNA sequences close to the lipid anchor, it is possible to vary the inter-membrane distance between the liposomes and thus to investigate the role of distances concerning fusion efficiency. In general, long DNA spacer increases docking rates and reduces fusion efficiency. This is in agreement with the requirement of close proximity for membrane fusion (Section 2.4). ${ }^{[104-107]}$ Another artificial fusion system also bases on lipid anchors. However, in this case the anchors are linked via flexible polyethylenglycol (PEG) spacers to peptidic coiled-coil forming recognition units. This construct resembles the minimal SNARE machinery concerning the coiled-coil forming recognition units of three heptads as the shortest known hetero coiled coil and fuse liposomes of different sizes without leakage. The application of LUVs and pore-spanning membranes for these model peptides leads only to docking. ${ }^{[88,108]}$ This is understandable, because the lipid anchor does not span the whole membrane and does not affect membrane continuity significantly. The force of molecular recognition might not be sufficiently transferred to the membranes via the lipid anchors to induce fusion of membranes with less curvature stress. Lipids presenting three melamine and three cyanuric acid residues in opposing membrane lead to liposome docking through hydrogen bonds but not to fusion. Latter is induced by addition of antimicrobial membrane surface-active magainin. ${ }^{[109]}$ This amphiphilic peptide forms an a-helix with a hydrophobic and a positively charged surface on opposite sides. Magainin binds to negatively charged lipid headgroups and disrupts membrane continuity. ${ }^{[110]}$ Concerning the SNARE proteins Syb2 and Sx1A it is suggested that the TMDs do not only function as membrane anchors but have further functions like the perturbation of lipid continuity or the induction of bending stress making membranes prone to fuse. ${ }^{[11,111]}$ Especially, in the late stages of the fusion process (fusion pore opening) TMDs are probably crucial factors. ${ }^{[14]}$ To shed more light on the functions of TMDs during the fusion process, 
fusogenes were developed which are combined constructs of natural LR/TMD sequences of Syb2 or Sx1A and artificial recognition motifs based on peptide nucleic acids (PNA) or coiled-coil forming peptides (Figure 2.6.2-1, C). ${ }^{[15,16]}$

\subsubsection{1) PNA-LR/TMD Peptide Hybrids as SNARE Mimetics}

The linkage of the LRs/TMDs of Syb2 and Sx1A (SybLR/TMD and SxLR/TMD) as native membrane anchors and aminoethylglycine peptide nucleic acid (aeg-PNA) decamers yielding SNARE mimetics which combine natural features of SNARE proteins with well controllable artificial recognition motifs. Unlike lipid based membrane anchors, TMDs are thought to play an active role during membrane fusion. After docking of the membranes is induced by molecular recognition, $N$-terminal regions including the LRs might perturb membrane packing and lead to the first hydrophobic contact of the opposing membranes through splayed lipids (Section 2.4). Furthermore, TMDs might induce electrostatic condensation of lipids and thus create a suitable lipidic environment for membrane fusion. Through $C$-terminal zippering mechanical force is transferred to the membrane allowing the formation of a fusion pore. Afterwards, TMDs reduce membrane curvature stress and thereby stabilising the pore. ${ }^{[12,67]}$ The aeg-PNA consists of a polyamide backbone of $\mathrm{N}$-(2-aminoethyl)glycine (aeg) units and nucleobases which are attached through methylenecarbonyl linkers to the backbone. ${ }^{[112]}$ The sequences for PNA decamers are chosen concerning hybridisation (WATSON-CRICK base pairing) in parallel and antiparallel orientation (Figure 2.6.2.1-1). The PNA strands form thermally very stable helical duplexes with melting temperatures of $46^{\circ} \mathrm{C}$ for parallel (PNA1/PNA3) and $66^{\circ} \mathrm{C}$ for antiparallel (PNA1/PNA2, released energy upon association is $31 \mathrm{kB} T$ ) duplexes. The hybridisation is independent of the prevailing salt concentration. ${ }^{[15,113-115]}$ The aeg-backbone is neutral and has no negative charges like RNA and DNA backbones. Furthermore, it is achiral and thus synthesis problems of PNA monomers concerning enantiomeric purity is avoided. PNA is highly biostable, i.e. it is not degraded by nucleases or proteases. ${ }^{[16]}$ In vitro experiments demonstrated that $4-5 \%$ of the SNARE complexes exhibit an antiparallel orientation. ${ }^{[117]}$ In addition to the ternary Syb2/Sx1A/SNAP 25 complex several other weakly interacting binary complexes like a Syb2/Sx1A-complex were identified. ${ }^{[7]}$ The PNA recognition system provides the possibility to investigate the relationship between 
recognition orientation and membrane fusion. Another important aspect of PNA-LR/TMD peptide hybrids is the transition region between the PNA recognition units and the LRs of Syb2 and Sx1A. The LRs are probably random-coils which adapt a-helical structures not before the zippering interactions reach these regions. ${ }^{[76]}$ It is doubtful, if PNA recognition is convenient to induce $C$-terminal zippering because the topology of the PNA helix is different compared to the coiled-coil four-helix bundle of the native SNARE motifs (compare Figure 2.6.2.1-1 with Figure 2.6.2.2-1).
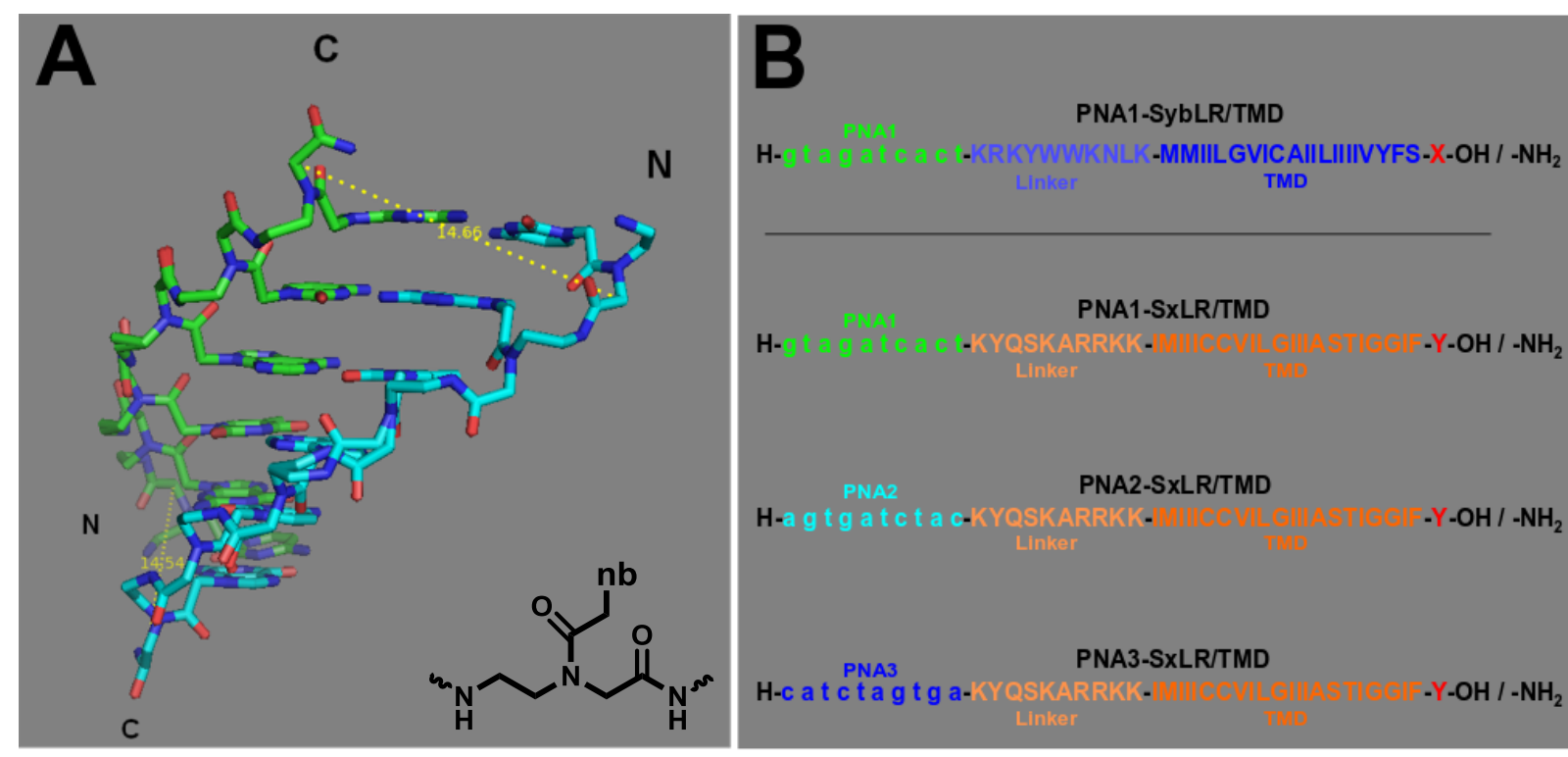

Figure 2.6.2.1-1 SNARE analogues based on the LRs/TMDs of Syb2 and Sx1A. The SNARE motifs are exchanged by decamers of aeg-PNA: A) Crystal structure of an antiparallel double helix of hexameric PNA strands. Green, red and blue sticks represent carbon, oxygen and nitrogen atoms, respectively. Letters $\mathrm{N}$ and $\mathrm{C}$ mark $\mathrm{C}$ - and $\mathrm{N}$-termini of the single strands. The distances between the terminal $\mathrm{C} \alpha$-atoms are $14.7 \AA$ (yellow dashed lines). The insert represents the basic structure of an aeg-PNA monomer (nb: nucleobase). B) Sequences of the used PNA-LR/TMD peptide hybrids: The LR/TMD of Syb2 (light and dark blue) is linked to the PNA1 sequence (light green). The LR/TMD of Sx1A is connected to the PNA1 (non-complementary, control), the PNA2 (cyan, antiparallel recognition) or the PNA3 sequence (dark blue, parallel recognition). Red $X$ and $Y$ mark the $C$-terminal aa positions of Syb2 and $S \times 1 A$ which were systematically mutated in the present study. The crystal structure is taken from ref. 113 (PDB code: 1pup) and was processed by using the PyMOL 0.99rc6 software.

For instance, the distance between the backbones of the PNA double helix is $\sim 15 \AA$ (Figure 2.6.2.1-1, A). The diameter of the Syb2/Sx1A-coil of the SNARE complex is $\sim 6 \AA$ in the transition region of the SNARE motifs and LRs (distance of the last $C$-ter- 


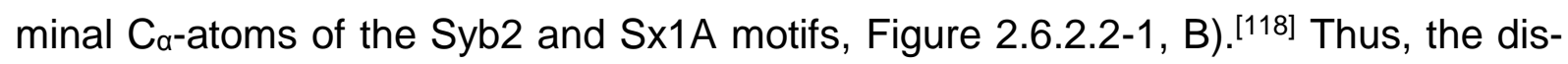
tance between the $C$-terminal aas of the Syb2 and Sx1A motifs is significantly smaller than the distance between the $C$-terminal PNA monomers of the artificial PNA recognition system. Moreover, the rotation per bp $\left(20^{\circ}\right)$ within the PNA-double helix differs from the twist of the Syb2 and Sx1A helices per aa within the Syb2/Sx1A-coil $\left(2.9^{\circ}\right.$, for calculation see Section 5.8).[119] These structural differences of the PNA recognition system lead to a different positioning of the $N$-terminal aas in the LRs of Syb2 and Sx1A. Consequently, the artificial PNA recognition units might impair $C$-terminal zippering interactions within the LRs and TMDs. Nevertheless, PNA-LR/TMD peptide hybrids are able to bring membranes in spatial proximity and to induce fusion. ${ }^{[15]}$ In the case of lacking $C$-terminal zippering, the transfer of mechanical force to the TMDs like in the case of native SNARE recognition is probably reduced, because the LRs of Syb2 and Sx1A do not form stiff $\alpha$-helices. Thus, the flexibility of the unstructured LRs impair effective force transfer and membrane fusion. ${ }^{[13]}$ The transferred force might nonetheless perturb membrane continuity by a motion of polar $C$-terminal ends of the TMDs from the membrane-water interface into the hydrophobic centre of the membrane and open a fusion pore. ${ }^{[14]}$ Thus, the required energy for this motion might be a crucial factor and depends on the $C$-terminal charges of the TMDs of Syb2 and Sx1A. ${ }^{[17]}$ The usage of native LR/TMD sequences offers the possibility to modify the LRs/TMDs of Syb2 and Sx1A: Mutation studies exchanging particular aas like in this work or a batch of aas can be performed as well as the introduction of tags and chemical modified aas as further possible applications. The advantage of these fusogenes is that modifications - whatever they look like - are in the natural peptidic environment. Thus, conclusions of studies using PNA-LR/TMD peptide hybrids are suitable to clarify the role of specific regions of the natural SNARE-TMDs right up to single atoms (precision depends also on the applied detection method).

\subsubsection{2) E3/K3-LR/TMD Peptide Hybrids as SNARE Mimetics}

MEYENBERG et al. developed artificial fusogenes which are similar to the already presented PNA-LR/TMD peptide hybrids. Again, the LR/TMD sequences of Syb2 and Sx1A are used, but they are coupled to coiled-coil forming peptides (E3 and K3) instead of PNA. ${ }^{[16]} \alpha$-helical coiled coils are well studied quaternary protein structures of 
right-handed $\alpha$-helices which form left-handed superstructures. ${ }^{[120]}$ The relationship between the aa sequence and the resulting quaternary structures like dimers, trimers or tetramers is well understood. Empirically found sequence-to-structure rules are used to create new artificial supermolecular structures (de novo design). Coiled coils are built up by heptad repeats with a typical pattern of hydrophobic ( $h$ ) and polar ( $p$ ) aa residues (hpphppp, usually denoted as abcdefg). Consequently, a coiled-coil sequence presents its hydrophobic residues (3.5-residue spacing) nearly concerted with one helical turn (3.6 residues per turn), i.e. the hydrophobic aa residues are on the same side of the $\alpha$-helix. However, the result of the slight difference in aa arrangement is the typical superhelical structure of coiled coils. Positions $\boldsymbol{a}$ and $\boldsymbol{d}$ are located the interface of the coiled-coil strands and connect the monomers by hydrophobic interactions (knob-into-holes $(\mathrm{KIH})$ interactions). ${ }^{[121]}$ These aas are important for the stability, oligomeric state and the orientation (parallel or antiparallel) of the coiled coils. For instance, if in positions $\boldsymbol{a}$ and $\boldsymbol{d}$ an isoleucine and a leucine residue are present, respectively, a parallel dimer is the preferred superstructure. The dimer formation can be supported by the introduction of asparagine in position a (accompanied by reduced stability). ${ }^{[122]}$ If the positions of isoleucine and leucine are exchanged against each other, a tetramer is preferred. [123] Positions $\boldsymbol{e}$ and $\boldsymbol{g}$ harbour typically charged aas like lysine, glutamate, aspartate or arginine and contribute to the stability and specificity of the coiled-coil structure beside the hydrophobic core interactions. The usage of oppositely charged aas in different peptide sequences for the positions $\boldsymbol{e}$ and $\boldsymbol{g}$ induces complementary and supports the formation of heterodimers. Amino acids in position $\boldsymbol{b}, \boldsymbol{c}$ and $\boldsymbol{f}$ are suitable to improve the water solubility of coiled-coil structures because of their peripheral location within the complex. However, these positions influence also the formation of higher oligomers (> trimers), if they are occupied by small hydrophobic aas (e.g. alanine). Furthermore, helix favouring aas like alanine are more frequent at these positions of coiled-coil sequences than helix breakers like glycine or proline, because helicity is the basic requirement of coiled-coil formation. In general, positions $\boldsymbol{b}$, $\boldsymbol{c}, \boldsymbol{e}, \boldsymbol{f}$ and $\boldsymbol{g}$ are more permissive than positions $\boldsymbol{a}$ and $\boldsymbol{d}$ for modifications. ${ }^{[24,125]}$ Following these relationships between sequence and structure, HODGE et al. developed a short and stable coiled-coil recognition system (Figure 2.6.2.2-1, C-E). The sequences E3 ((EIAALEK $\left.)_{3}\right)$ and K3 ((KIAALKE) $\left.)_{3}\right)$ form heterodimeric parallel coiled coils. Positions $\boldsymbol{a}$ and $\boldsymbol{d}$ are occupied by isoleucine and leucine which support dimerisation. In 
the case of isoleucine, the hydrophobicity at the interaction site and therefore dimer stability is increased. In positions $\boldsymbol{b}$ and $\boldsymbol{c}$ two alanines reside which enhance helicity. Oppositely charged aas are localised in $\boldsymbol{e}$ and $\boldsymbol{g}$ (glutamate in E3 and lysine in K3), which improves heterodimerisation. Three heptad repeats are sufficient to form stable duplexes with a $K_{d}$ value of $70 \mathrm{nM}$ (released energy upon association is $16 \mathrm{kB} T$ ). ${ }^{[126]}$ Due to the coiled-coil structure, the E3/K3 recognition system corresponds more to the native SNARE recognition than the PNA system. The diameter of the E3/K3-coiled coil is $\sim 1.4 \mathrm{~nm}(14 \AA)$ and match the SNARE complex diameter. ${ }^{[118]}$ However, the diameter of the Syb2/Sx1A-coil is with $\sim 0.6 \mathrm{~nm}(6 \AA)$ much smaller (Figure 2.6.2.2-1, B-C).
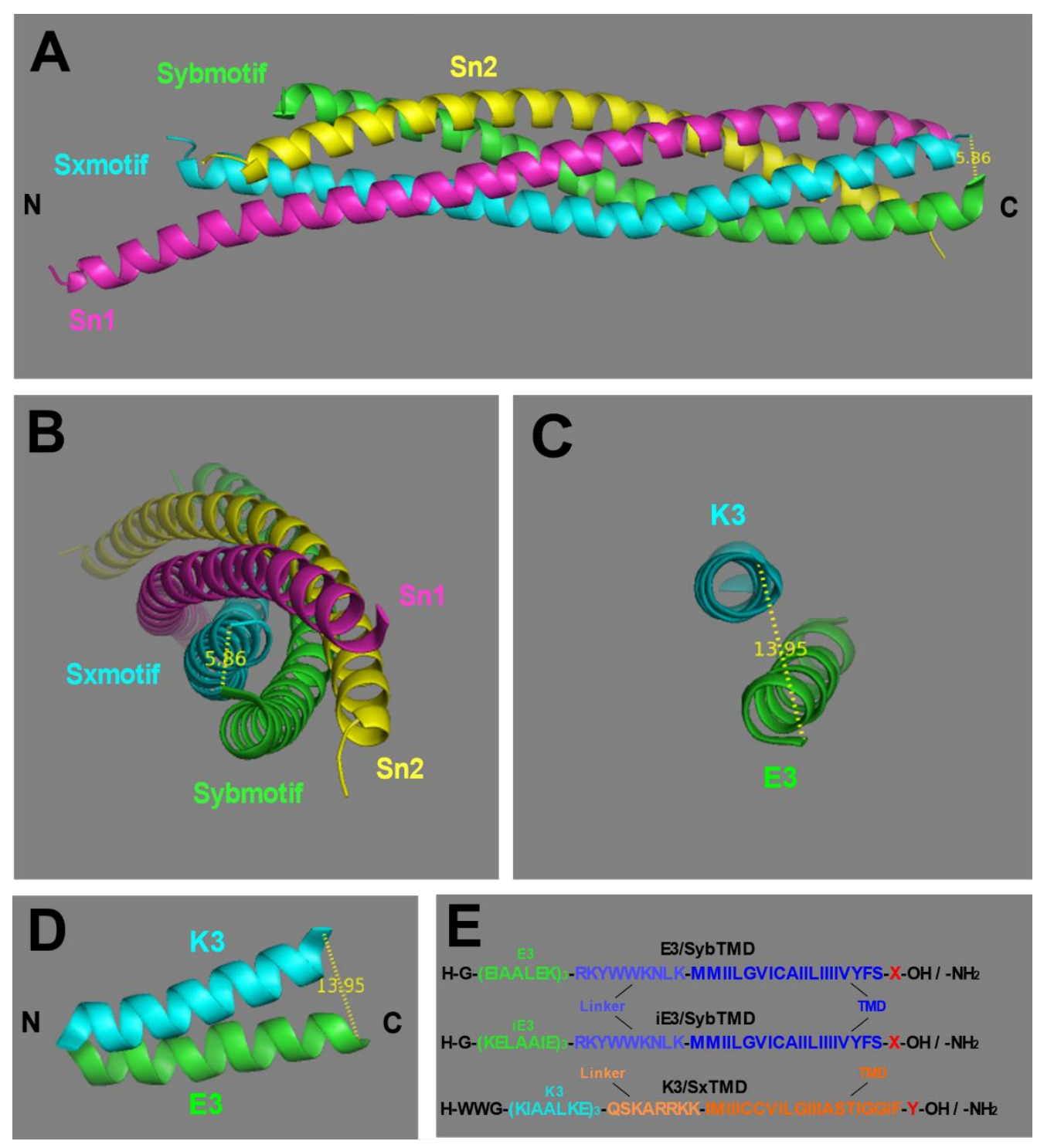

Figure 2.6.2.2-1 Comparison of structural features of the natural SNARE complex and the E3/K3 complex: A) Lateral view on the SNARE complex with the four $\alpha$-helices of Syb2 (Sybmotif, green), Sx1A (Sxmotif, cyan) and the two SNAP 25 domains (Sn1, magenta and Sn2, yellow). $\mathrm{N}$ - and $C$-terminus of 
the complex are tagged by the letters $\mathrm{N}$ and $\mathrm{C}$, respectively. B) Frontal view on the $C$-terminal end of the SNARE complex. (colour code as in A). The distance between the last $C$-terminal Ca-atoms is $5.9 \AA$ (yellow dashed line). C) Frontal view on the $C$-terminal end of the $E 3 / K 3$ complex (E3, green and K3, cyan). The distance between the last $C$-terminal Ca-atoms is $14.0 \AA$ (yellow dashed line). D) Lateral view on the E3/K3 complex (colour code as in C). E) Amino acid sequences of the used SNARE analogues: The LR/TMD (light and dark blue) of Syb2 is connected to the E3 recognition unit (green). In the case of the inverted E3 peptide (iE3), the aa sequence is reverse. The LR/TMD (light and dark orange) of $\mathrm{S} \times 1 \mathrm{~A}$ is connected to the $\mathrm{K} 3$ recognition unit (cyan). Red $\mathrm{X}$ and $\mathrm{Y}$ mark $C$-terminal aa positions of the TMDs which were systematically mutated in the present study: Crystal and NMR solution structures are taken from ref. ${ }^{[127]}$ (SNARE complex, PDB code: 1n7s) and ref. 120 (E3/K3 complex, PDB code: 1u0i). Structures were processed by the PyMOL 0.99rc6 software.

The twists per aa of the E3/K3 and Syb2/Sx1A coiled coils coincide (both $2.9^{\circ}$, for calculation see Section 5.8). Therefore, the structural features of E3/K3-LR/TMD peptide hybrids match those of native Syb2 and Sx1A better compared to the PNA-LR/TMD peptide hybrids and the situation for $C$-terminal zippering is more convenient. However, a direct evidence (e.g. due to crystal structures) for zippering up to the $C$-termini is lacking. In addition, differences in the heptad register are also present. The $C$-terminal heptads of Syb2 and Sx1A reach into the LRs and this is not the case for the artificial E3/K3 based model peptides. $C$-terminal zippering requires spatial proximity of the aas in the LRs/TMDs which depends on the aa arrangement induced by the recognition units (see the Appendix, Comparison of Native and Artificial Sequences, Figure A-8). Modifications of the $C$-termini of E3-SybLR/TMD and K3-SxLR/TMD model peptides are identical to those of the PNA-LR/TMD peptide hybrids (for a more detailed description of modifications see Section 2.7). 


\subsection{3) Detection Methods for Membrane Characteristics}

The following section describes detection techniques for the determination of membrane characteristics. The focus is on the detection of membrane adhesion (docking), the mixing of lipids between membranes (fusion of membrane leaflets), the exchange of contents between membrane encapsulated compartments through an aqueous connection (fusion pore) and the increase of membrane size. These are the key characteristics for membrane fusion. ${ }^{[108]}$ Beside the key characteristics of fusion, there are other important membrane features which should be known for the evaluation of membrane fusion. For instance, the lamellarity of liposomes, the orientation of proteins within the membrane, the lateral mobility of membrane components and the phase behaviour of the membrane are important features with regard to membrane fusion.

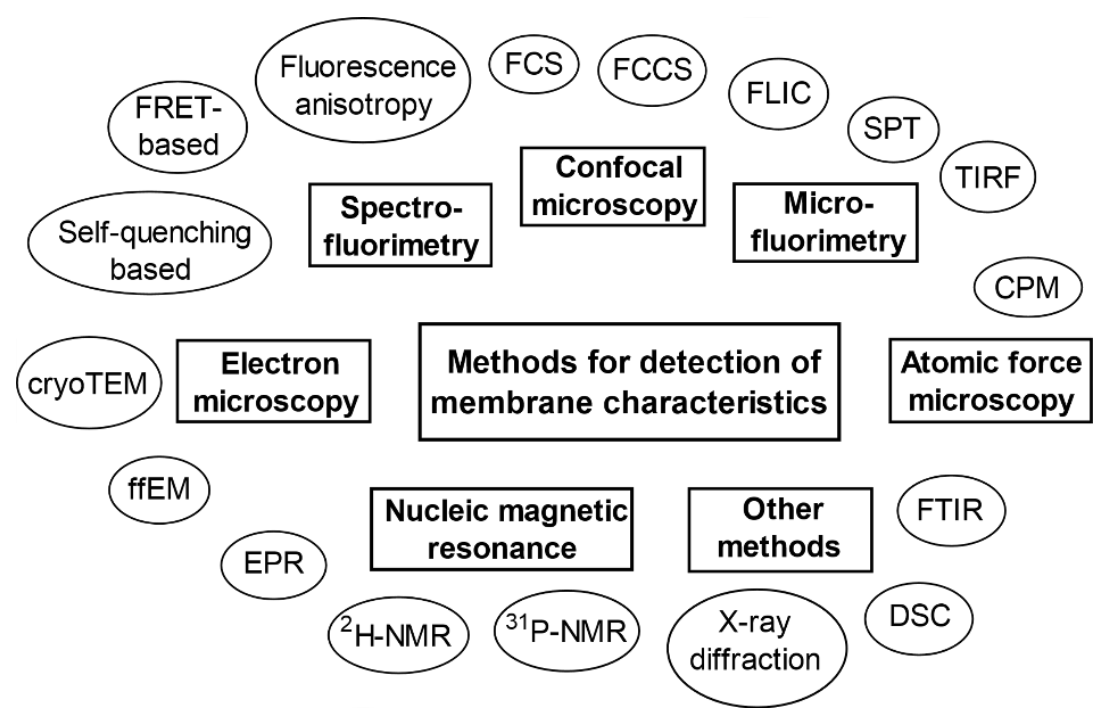

Figure 2.6.3-1 Overview of the variety of methods applied to determine membrane characteristics. Electron paramagnetic resonance (EPR): Orientation of spin-labelled proteins within a membrane; phase separation of membranes. ${ }^{[128,129]}$ Nuclear magnetic resonance spectroscopy $\left({ }^{31} \mathrm{P}-,{ }^{2} \mathrm{H}-\mathrm{NMR}\right)$ : Lamellarity of liposomes; phospholipid ratio between inner and outer leaflet; orientation of cholesterol within a phospholipid bilayer. ${ }^{[130,131]} X$-ray diffraction: Structural rearrangements of lipids during phase transition; detection of membrane fusion intermediates. ${ }^{[45,132]}$ Differential scanning calorimetry (DSC): Influence of lipids on membrane phase transition. ${ }^{[133]}$ Fourier transform infrared spectroscopy (FTIR): Conformations in acyl chains of lipids in different phases (lipid packing). ${ }^{[134]}$ The present collection of methods does not claim completeness. Furthermore, it is not possible to differentiate methods clearly from each other, because they are often applied in combination. 


\subsubsection{1) Electron Microscopy}

Electron microscopy (EM) is a very useful detection tool, because it delivers images of liposomes. EM images deliver important structural information within the nanometer range regarding size and membrane integrity. Moreover, it is possible to observe directly membrane fusion intermediates. Two examples are freeze fracture EM (ffEM) which yields three-dimensional images of liposomes and cryogenic transmission EM (cryoTEM) by which a through-vision-perspective is obtained. ${ }^{[21,135,136]}$ However, a serious drawback of ffEM concerning heterogeneous liposome populations is that fracture planes pass through randomly distributed liposomes. Thus, the planes do not represent the midplane of liposomes in any case. Consequently, diameters obtained by ffEM are partly smaller than the actual diameters. ${ }^{[32]}$ The drawback can be overcome by using cryoTEM. In this method, a liquid film of $\sim 300 \mathrm{~nm}$ thickness is frozen and irradiated in its entirety. Liposomes within the film can be recorded. Due to ice crystal formation and elevated osmotic pressure in both EM methods, morphological shifts are conceivable. Therefore, the application of cryoprotectants like DMSO are important. ${ }^{[136]}$

\subsubsection{2) Microfluorimetry}

Microfluorimetry (MF) represents an important tool for the detection of membrane characteristics in the sub-micron range. This detection method relies on confocal microscopy and its technical advancement confocal laser scanning microscopy (CLSM). In both techniques small areas of fluorophore labelled membranes are excited. The emission signal is observed via an optical microscope supported by a photomultiplier. ${ }^{[137]}$ Total internal reflection fluorescence (TIRF) microscopy is one widely used MF technique. When a laser beam illuminates the interface of two layers with different refractive indices (glass and water), total reflection occurs. However, some of the light energy permeates the interface of the two layers and creates an electric field parallel to the layers (evanescent wave). This field is capable of exciting fluorophores which are close to the layer with the higher refractive index (glass). Consequently, only fluorescent molecules within distances of $100 \mathrm{~nm}$ to the surface are excited. The emission is detected in good spatial and temporal resolution by a microscope equipped with a CCD camera. ${ }^{[138]}$ For instance, it is possible to discern individual labelled lipids during the 
fusion process and to distinguish between different intermediates of single fusion events. ${ }^{[52]}$ In general, by using TIRF one can obtain kinetic binding curves or binding isotherms of membrane-protein interactions. ${ }^{[7]}$ For fluorescence recovery after photobleaching (FRAP) microscopy, the membrane is labelled with a fluorophore and a defined area of the membrane is irradiated by a laser beam which induces photobleaching of the fluorophores. After bleaching, the recovery of the fluorescence in the irradiated region is monitored. FRAP enables the determination of lateral diffusion coefficients of lipids and proteins within the membrane. ${ }^{[139]}$ Single particle tracking (SPT) is also used to detect diffusion processes. Using this method, labelled molecules are not bleached but tracked by cameras with suitable resolution. Fluorescence interference contrast (FLIC) microscopy exploits the interference of direct and reflected light from fluorophores in front of a mirror. It is possible to determine protein orientation within lipid bilayers with a resolution of 1-2 nm, if different positions of the protein are labelled. ${ }^{[77]}$

\subsubsection{3) Spectrofluorimetry}

Spectrofluorimetry (SF) is closely related to MF. Instead of images or videos, fluorescence spectra are recorded and analysed. Fluorescence correlation spectroscopy (FCS) is a method for the detection of molecular binding events and lateral diffusion. Through the usage of two dyes of a FRET (Förster resonance energy transfer) pair it is possible to discriminate between docking and fusion of two liposomes with temporal resolution in the millisecond range (fluorescence cross correlation spectroscopy (FCCS)). Two populations of liposomes labelled with different dyes diffuse freely in a confocal microscope. If liposomes interact with each other, equal intensive fluorescence signals are temporal correlated but no FRET effect is observed. When leaflet mixing between the two differently labelled liposomes occurs, the intensity of one signal is enhanced (acceptor fluorophore) and the other signal (donor fluorophore) decreases. ${ }^{[140-142]}$ The effect of fluorescence anisotropy is exploited to detect binding events between proteins, e.g. between membrane bound and cytosolic proteins or between proteins which tend to aggregate at membranes. ${ }^{[9,143]}$ Fluorophores are attached to one binding partner. The fluorophores are excited by polarised light and the generated emission depends on the rotational time of the protein bound fluorophore. The 
rotational time is slowed down by binding partners and this causes less depolarisation of the emitted light compared to the polarisation of the exciting light. The reduction of depolarisation indicates binding events. ${ }^{[144]}$

Spectrofluorimetry in bulk assays based on FRET: The detection and analyses of FRET between headgroup labelled lipids in membranes is exploited to determine leaflet mixing between separated membranes which is induced by membrane fusion (Figure 2.6.3.3-1, B). FRET is a physical process of electronic energy transfer between a donor and an acceptor fluorophore. The donor is excited by light irradiation. Thereby, it transfers energy to the acceptor, which emits light at another wavelength. At the same time emission of the donor is inhibited. FRET is independent of direct contact or emitted light of the donor. Rather the transfer requires dipol-dipol interactions of the fluorophores and depends strongly on the distance between the donor and acceptor fluorophore (Figure 2.6.3.3-1, A). ${ }^{[145,146]}$ Typical Förster radii (distance between donor and acceptor with a FRET efficiency of $50 \%$ ) are in the range of $1.5-6.0 \mathrm{~nm} \cdot{ }^{[147]}$

A

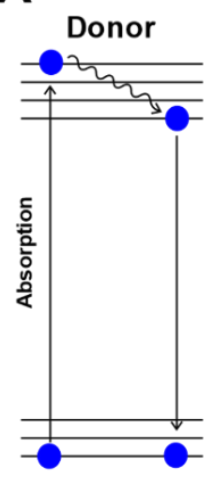

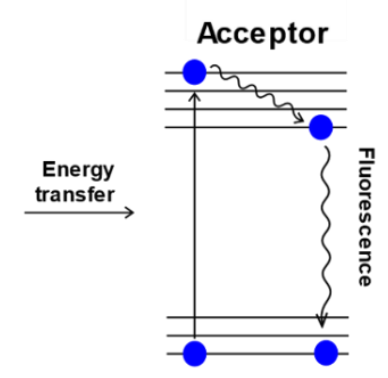

B

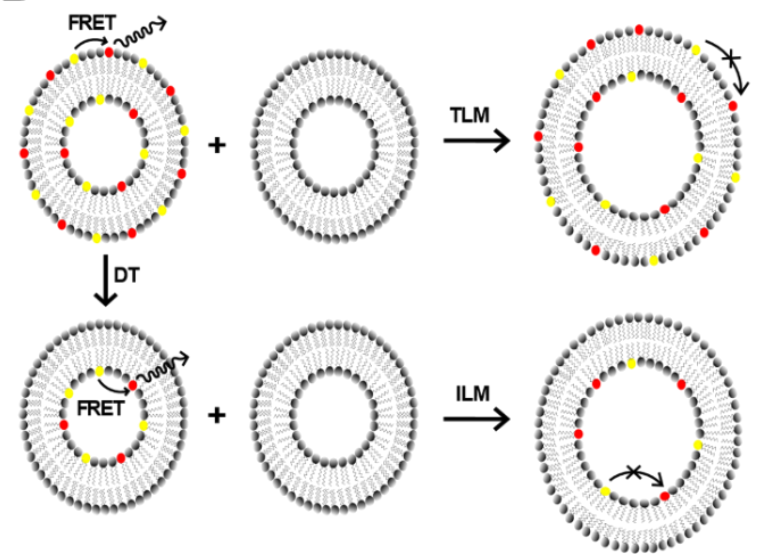

Figure 2.6.3.3-1 FRET based leaflet mixing assays: A) FRET effect illustrated by a Jablonski diagram (taken from ref.146). B) Total leaflet mixing (TLM, top) and inner leaflet mixing (ILM, bottom) after treatment of labelled liposomes (NBD as donor (yellow) and Rh as acceptor (red)) with sodium dithionite (DT).

There are two methods for detecting leaflet mixing: One membrane is labelled with a FRET pair (e.g. nitrobenzoxadiazol (NBD) as donor and lissamine rhodamine B (Rh) as acceptor). If these membranes are in contact with unlabelled membranes, leaflet mixing might occur, i.e. leaflets of apposed membranes merge. Thus, labelled lipids 
migrate into the unlabelled region of the newly formed membrane and vice versa. Consequently, labelled lipids are diluted and thus the FRET efficiency is reduced. Usually while exciting the donor fluorophore, the emission of the donor fluorophore is detected and increases when the FRET efficiency is reduced by dilution of labelled lipids (dequenching of donor). ${ }^{[148]}$ By treating liposomes with dithionite ions (membrane impermeable substance), NBD of the outer leaflets is deactivated. As result, the reduction of the FRET effect by dilution of labelled lipids during membrane fusion is restricted to the inner leaflets (Figure 2.6.3.3-1, B). In conclusion, hemifusion (merger of outer leaflets) and full fusion (merger of both leaflets) is distinguishable by total and inner leaflet mixing assays exploiting the increase of NBD fluorescence due to FRET reduction (Figure 2.6.3.3-2). ${ }^{[149,150]}$

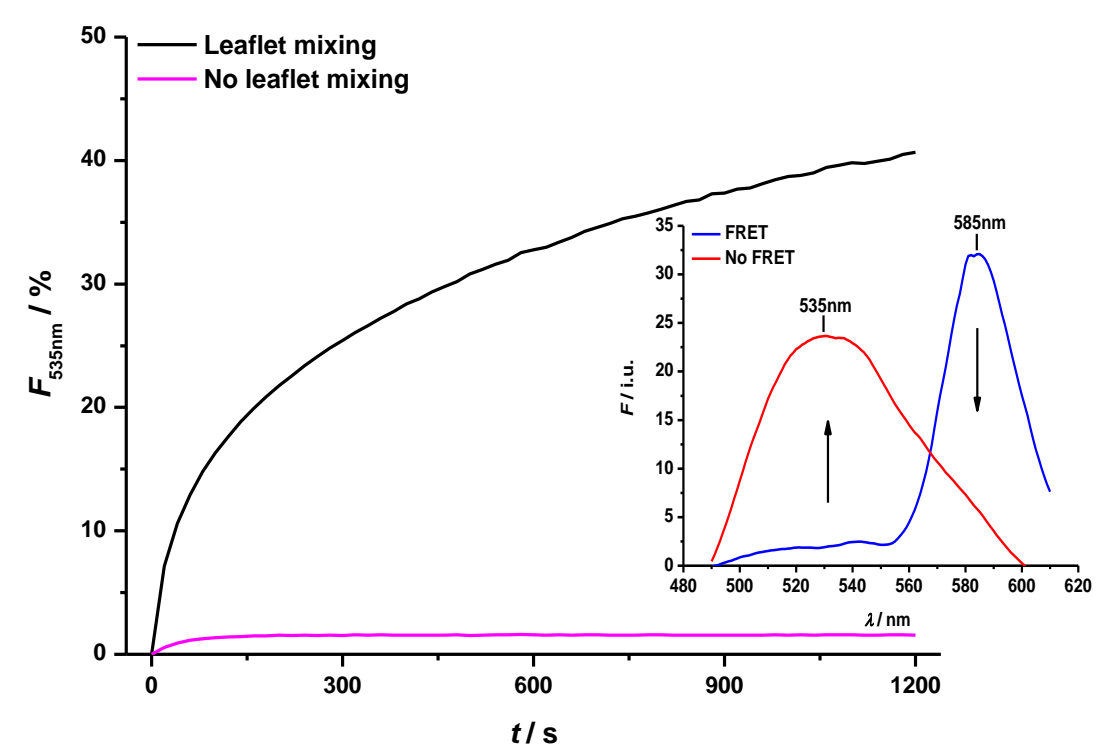

Figure 2.6.3.3-2 Fluorescence spectra of a typical leaflet mixing experiment with FRET fluorophores in one liposome population. Fusion with unlabelled liposomes results in an increase of the donor emission (NBD at $535 \mathrm{~nm}$, indicated by the arrow below the red curve) and in a decrease of the acceptor emission ( $R h$ at $585 \mathrm{~nm}$, indicated by the arrow below the blue curve).

The second FRET based assay for the detection of leaflet mixing relies on the labelling of both membranes with only one fluorophore, respectively. (e.g. Texas Red and Oregon Green). If leaflet mixing takes place, both dyes approach each other and an increase of the FRET effect is observed (not depicted). ${ }^{[151]}$ Through this experimental arrangement failed results due to liposome rupture processes are avoided, because FRET occurs only when the membranes merge. In the case of two fluorophores in one liposome population (Figure 2.6.3.3-1, B), the rupture of liposomes leads to FRET pair 
dilution, i.e. the detected reduction of FRET efficiency is not necessarily related to membrane fusion.

Spectrofluorimetry in bulk assays based on self-quenching and fluorescent complexes: Further indication for full fusion is gained by content mixing assays. Water-soluble self-quenching fluorophores (e.g. sulforhodamine B (SRB) or calcein) or fluorescent complex forming substances (terbium cations and dipicolinic acid (DPA)) are used for this purpose. ${ }^{[152-154]}$ When liposomes loaded with SRB in self-quenching concentrations are mixed with liposomes without SRB, the concentration of the dye is reduced and an increase in emission is detected. This implies an aqueous connection between the liposomes. However, an increase in emission can also occur due to leakage processes. ${ }^{[155]}$ Therefore, a control experiment must be performed using liposomes which are filled with a content dye, but do not carry fusogenes in their membranes. In another experimental setup, one liposome population carries terbium ions and the other population DPA. Upon aqueous contact between both populations, a fluorescent complex is formed and its emission can be detected. Leakage during the process of fusion is also a problematic issue for this content mixing assay and must be reviewed by an control experiment. If the membrane-impermeable strong chelator ethylendiaminetetraacetate (EDTA) is added to liposomes after starting the fusion reaction with sufficient time delay, terbium ions are chelated by EDTA and a decrease in emission is detected when leakage occurs. ${ }^{[154]}$

\subsubsection{4) Colloidal Probe Microscopy}

Membrane-membrane interactions can be investigated by modified atomic force microscopy (AFM). The standard tip of a cantilever has a sharp pyramidal shape inappropriate for the coverage with a lipid bilayer. Therefore, in colloidal probe microscopy (CPM) the cantilever is equipped with a colloidal probe made of borosilicate glass with diameters of 1-20 $\mu \mathrm{m}$. The spherical probe is covered by a lipid bilayer with integral peptides. An opposing solid supported membrane on a silicon wafer is also doped with peptides. The integrity of membranes on the sphere and the solid support is controlled by means of fluorescence microscopy. Homogeneous coverage and lateral mobility of the membrane are ascertained by CLSM based FRAP experiments. The cantilever 
with the spherical probe approaches the solid supported membrane. Through deflections of the cantilever acting forces are recorded. The approach curve contains information about breakthrough and fusion events. Afterwards, the cantilever is pulled away from the solid supported membrane. The resulting retraction curve gives information about adhesion forces induced by interacting peptides and merged leaflets of the membranes (Figure 2.6.3.4-1).

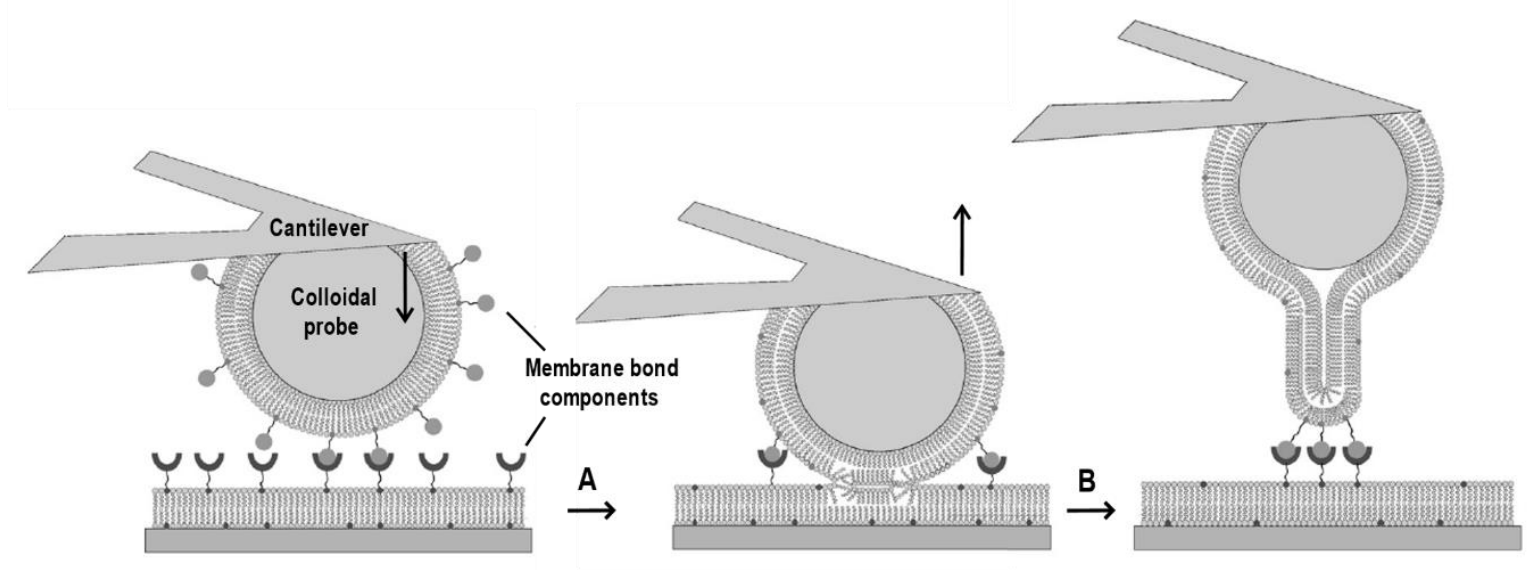

Figure 2.6.3.4-1 Illustration of a CPM experiment: A) Colloidal probe covered by a membrane approaches a solid supported membrane. B) Spherical probe retracts from the solid supported membrane. Interactions between membrane bound components (e.g. integral peptides) and membrane fusion can be detected (only hemifusion is depicted) (based on ref. 156).

The area of contact between both membranes can be estimated by the applied external force during the approach, the size of the probe and YounG's modulus $E$ of the corresponding materials. With knowledge of the cross sectional area of lipids it is possible to calculate how many lipids are in close contact. ${ }^{[156]}$ The estimation of the number of interacting peptides is more sophisticated because of possible peptide clustering. However, if one assumes a homogeneous distribution of peptides with known cross sectional area and molar peptide to lipid ratio, it is in principle possible to estimate the number of potentially interacting peptides. Thus, forces delivered by CPM can be assigned to single molecules. 


\section{7) Applied Membrane Fusion System}

In the present study SNARE mimetics consisting of natural LR/TMD sequences of the SNARE actors Syb2 and Sx1A are used. The native recognition motifs are replaced by artificial recognition units based on aeg-PNA or coiled-coil forming peptides (E3 and K3) (Sections 2.6.2.1 and 2.6.2.2). The peptides are synthesised by means of standard solid phase peptide synthesis (SPPS) using the base labile Fmoc-protecting group for $\mathrm{N}$-terminal amino groups and acid labile groups for the aa residues and the nucleobases of the PNA recognition units (Figure 2.7-1). ${ }^{157,158]}$ The synthesis principle is identical for all synthesised peptides, but differs slightly concerning the used reagents for the PNA-LR/TMD and E3/K3-LR/TMD peptide hybrids.

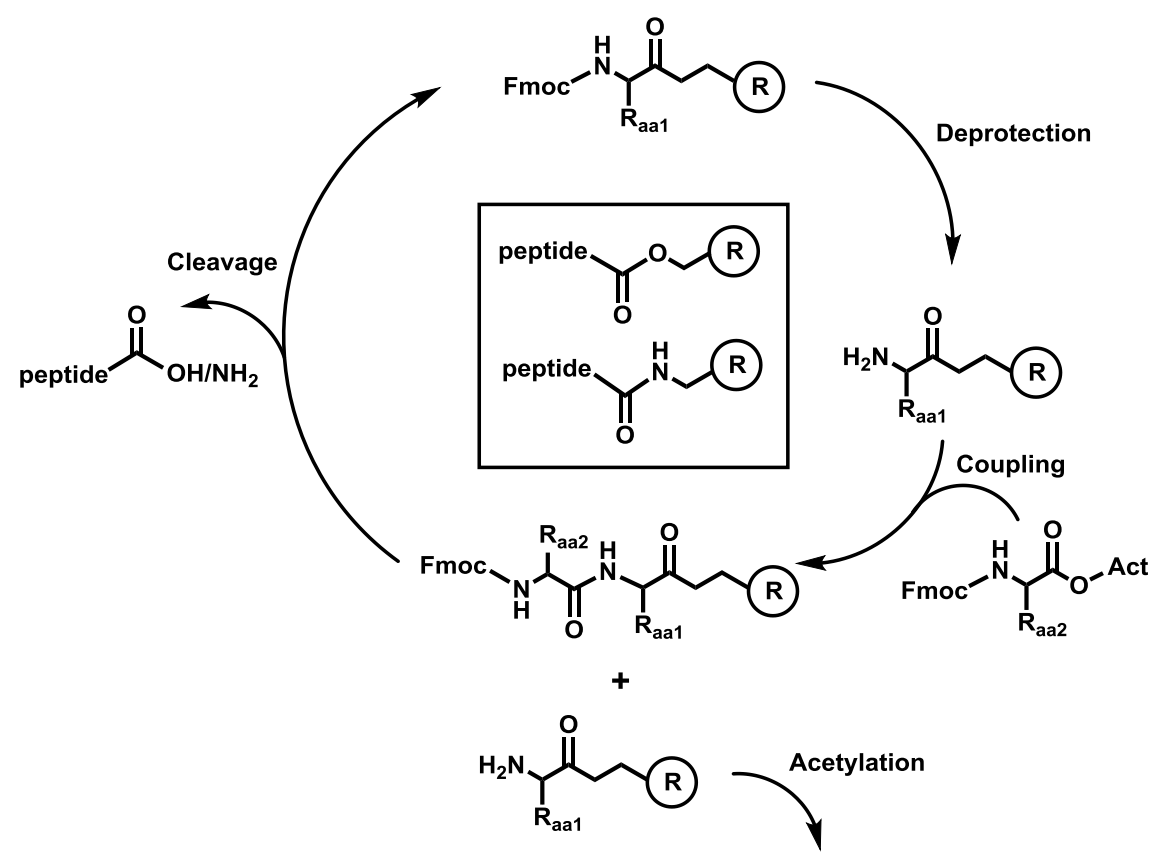

Figure 2.7-1 General synthesis scheme of Fmoc based SPPS: Framed structures emphasise the usage of different resins for $C$-terminal carboxy acid or amide groups, respectively. Deprotection of the $\mathrm{N}$-terminal Fmoc group is performed under basic conditions. For the formation of an amide bond (coupling) the introduced aa is activated through an active ester. By $N$-terminal acetylation (capping) of unreacted peptides, the misincorporation of aas during subsequent SPPS is avoided. Cleavage of the ester or amide bond between the peptide and resin is performed under acidic conditions releasing the respective $C$-terminal group and the aa side chains. Detailed presentations of deprotection, active ester formation, acetylation, cleavage with scavengers, aspartimide formation and racemisation reactions are available in the Appendix, Solid Phase Peptide Synthesis. Abbreviations: R: resin, Raa: aa residue and Act: active ester (based on ref. 159 and 160). 
The LR/TMD of Syb2 is throughout linked to PNA1 in the case of PNA-LR/TMD peptide hybrids and to $\mathrm{iE3}$ or $\mathrm{E} 3$ sequences in regard to E3/K3-LR/TMD peptide hybrids. On the other side, the LR/TMD of SX1A is connected to the sequences PNA1, PNA2 or PNA3 concerning PNA-LR/TMD peptide hybrids and to $K 3$ in the case of E3/K3-LR/TMD peptide hybrids (Figures 2.6.2.1-1 and 2.6.2.2-1). Interactions between PNA1-SybLR/TMD and PNA2-SxLR/TMD leads to antiparallel PNA duplex formation ( $C$ - and $N$-termini are positioned at opposite sides of the duplex). Parallel recognition ( $C$ - and $N$-termini are positioned on the same side of the duplex) is achieved by PNA1-SybLR/TMD and PNA3-SxLR/TMD and the combination of PNA1-SybLR/TMD and PNA1-SxLR/TMD serves as control with non-complementary recognition units. If E3-SybLR/TMD is combined with K3-SxLR/TMD parallel coiled-coil formation is expected. For mixtures of iE3-SybLR/TMD and K3-SxLR/TMD antiparallel interactions are assumed. ${ }^{[161]}$ The native $C$-terminal aas of SybLR/TMD and SxLR/TMD are threonine and glycine, respectively. These aas are exchanged for glutamate or lysine as carboxylic acids or amides (Table 2.7-1). The mutations result in different charges at the $C$-termini. The charges are suggested to influence the fusion behaviour with regard to membrane perturbation through the immersion of the $C$-terminal regions of the SybTMD and SxTMD into the hydrophobic core of the membrane. It is assumed that immersion of the $C$-terminal parts occurs during the recognition process of native SNARE proteins. ${ }^{[17,18,67]}$ Herein applied model peptides are used to gain a closer insight on this process. 
Table 2.7-1 Molecular structures of aas used for $C$-terminal modifications of the SybTMDs and SxTMDs. Inserted tables indicate charges $(Q)$ at the $C$-termini and applied abbreviations of $C$-terminally modified SNARE TMDs.

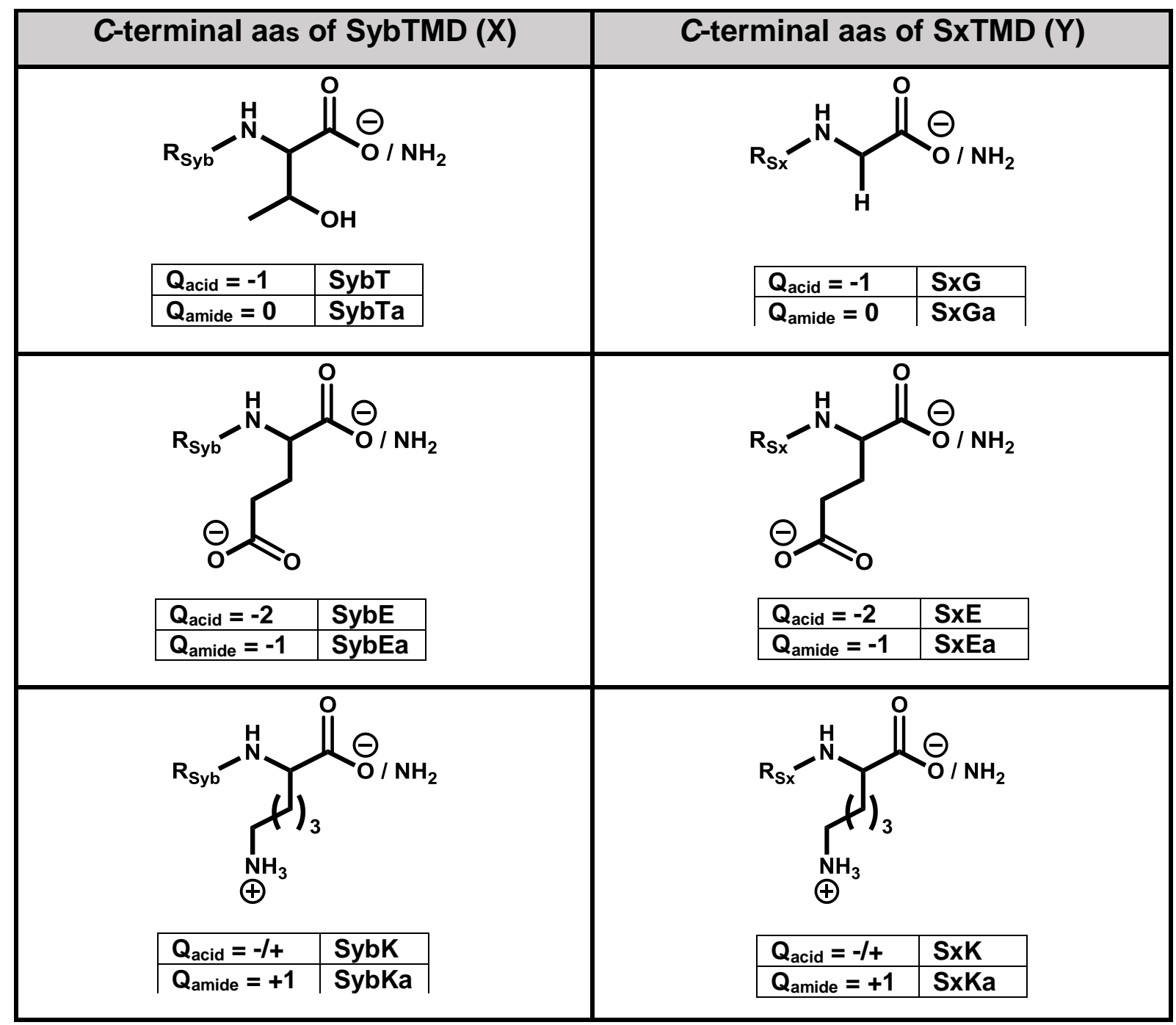

In addition to $C$-terminal charges, the orientation (parallel or antiparallel) of the SNARE mimetics during recognition plays a crucial role, because only the parallel orientation leads to $C$-terminal zippering. ${ }^{[15]}$ Molecular simulations suggested a thinning-widening mechanism for native SNARE proteins during the stalk-pore transition. $C$-terminal attractive forces between the TMDs of Syb2 and Sx1A as well as interactions between the LRs of the same peptides and membrane surfaces might combine thinning with widening motions of the stalk intermediate driven by $C$-terminal zippering. Those interactions might also inhibit the propagation of a metastable hemifusion diaphragm intermediate by clamping the stalk intermediate (Figure 2.7-2). ${ }^{[12,13]}$ 
A
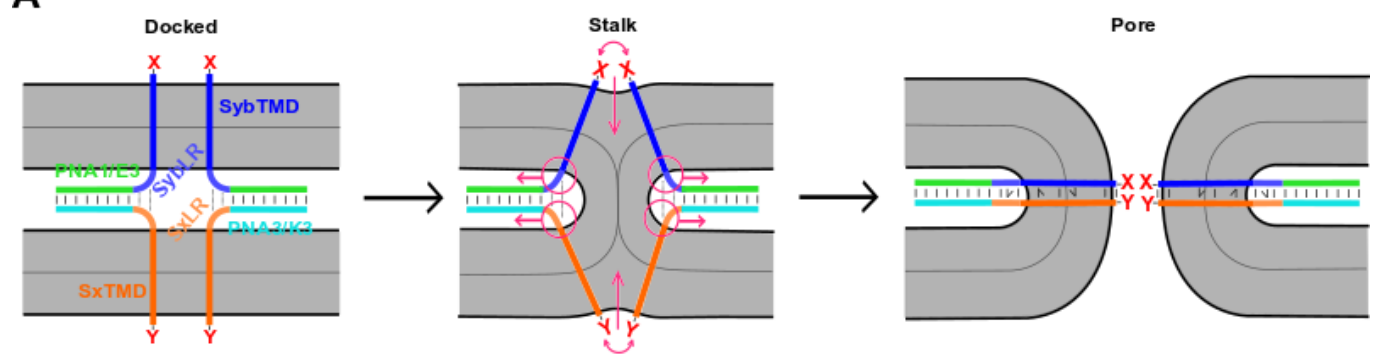

B
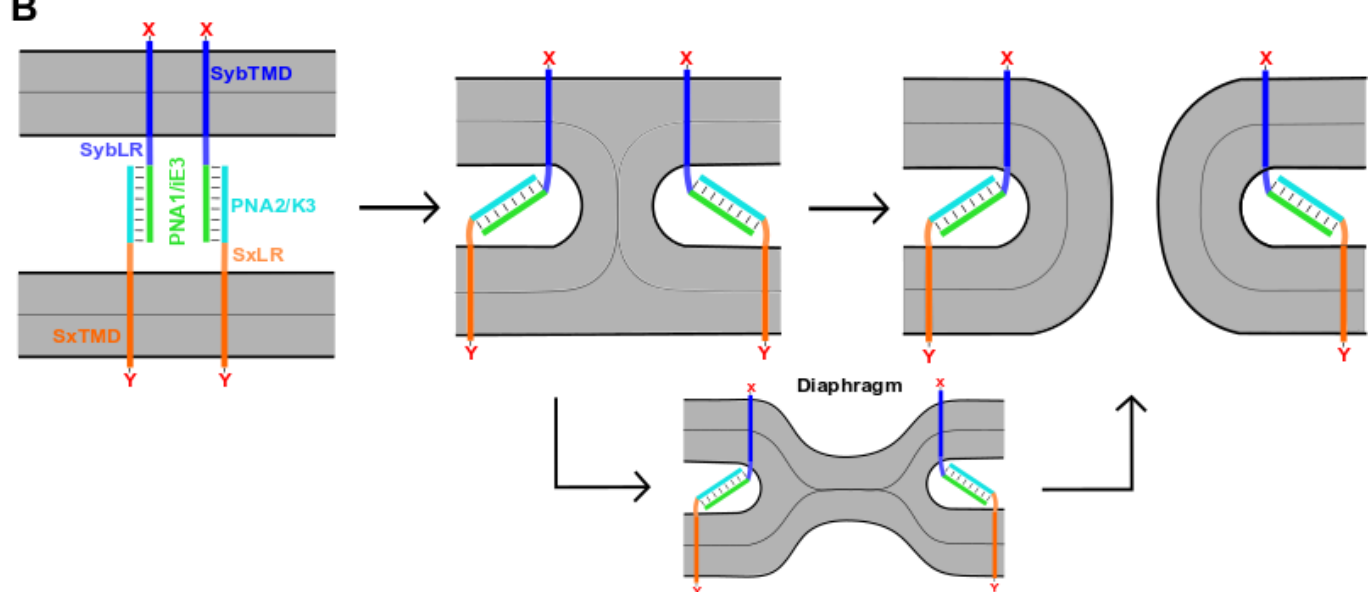

Figure 2.7-2 Illustration of the applied membrane fusion system with supposed fusion pathways: A) Parallel recognition between PNA1-SybLR/TMD and PNA3-SxLR/TMD or E3-SybLR/TMD and K3-SxLR/TMD corresponds to the native recognition orientation. Through $C$-terminal attractive forces between the duplexes (bent magenta arrows) and interactions between the LRs and membranes (magenta circles), the stalk intermediate thins down and widens simultaneously (magenta arrows). Thereby, fusion pore formation is achieved. Red $X$ and $Y$ indicate the positions of aa exchanges (Table 2.7-1). B) Antiparallel recognition between PNA1-SybLR/TMD and PNA2-Sx/LR/TMD or iE3Syb-LR/TMD and K3-SxLR/TMD leads to docking, but neither $C$-terminal zippering nor a thinning-widening mechanism is conceivable. The stalk intermediate might transit a diaphragm intermediate or emerge directly into a fusion pore.

Described SNARE mimetics are incorporated into LUVs, which are prepared by the extrusion technique. ${ }^{[93]}$ Thereby, peptide/lipid films are prepared from organic solutions. The films are hydrated before extrusion. The loss of lipid material during liposome preparation is controlled by determination of the amount of phosphate. Therefore, the lipids must be digested. The released phosphate is treated with molybdate under reductive conditions. The concentration of the resulting phosphatemolybdate complex is determined photometrically. ${ }^{[162,163]}$ Another easier and faster possibility to determine the lipid loss is to measure the decrease of fluorescence of labelled lipids 
before and after liposome preparation. However, this can only be performed for labelled liposomes. The insertion of peptides is controlled by a density-gradient centrifugation based assay. Thereby, the liposomes with peptides (proteoliposomes) are ultra-centrifuged using a density gradient. The equilibrium of gravity force and Brownian motion leads to separation of particles with high density (peptides) and those with low density (proteoliposomes). The separation is controlled by sodium dodecyl sulfate polyacrylamide gel electrophoresis (SDS-PAGE) and silver staining. The fusion behaviour of liposomes, which carry SNARE mimetics is examined by recording the course of FRET efficiency. For that, one proteoliposome population is labelled with the FRET pair NBD/Rh. If the labelled lipids are present in sufficient concentrations, FRET occurs due to close contact between the dyes. Leaflet mixing with unlabelled proteoliposomes lead to reduction of the FRET efficiency indicating for membrane fusion (Section 2.6.3.3). Moreover, SNARE mimetics were incorporated into pore spanning and solid supported membranes to investigate the fusion behaviour by CLSM and CPM (Sections 2.6.1 and 2.6.3.4). For experimental details see the experimental part (Chapter 5). 


\section{3) Results and Discussion}

\section{1) Influence of the Recognition Units of SNARE Mi- metics on Leaflet Mixing}

Both PNA-LR/TMD and E3/K3-LR/TMD peptide hybrids with modified $C$-termini were tested by FRET based bulk leaflet mixing assays regarding their ability to induce membrane fusion. Independent of the $C$-terminal modifications, E3/K3-LR/TMD peptide hybrids exhibit consistently stronger leaflet mixing than the corresponding PNA based model peptides (Figure 3.1-1, A-B).

A

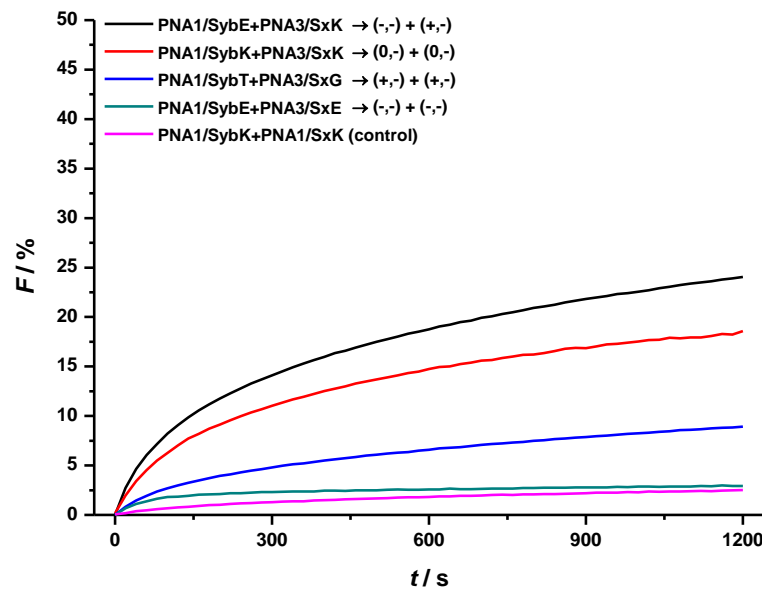

B

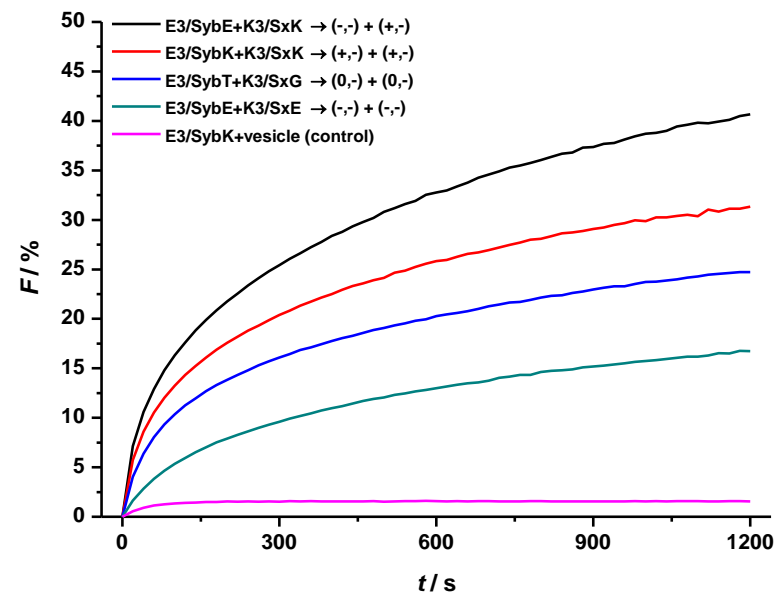

Figure 3.1-1 Total leaflet mixing assays of liposomes decorated with SNARE mimetics carrying carboxylic acid termini (SybX-and SxY-TMDs). The increase of donor emission (NBD) occurs due to mixing of lipids of the outer and inner leaflets: Parallel oriented recognition of $\mathbf{A}$ ) the PNA1/PNA3 duplex and $\mathbf{B}$ ) the E3/K3 coiled-coil formation. Native $C$-terminal threonine of the SybT-TMD and glycine of the SxGTMD were exchanged either by lysines (SybK- and SxK-TMDs), by glutamates (SybE- and SxE-TMDs) or by glutamates for the SybT-TMD and lysines for the SxG-TMD (SybE- and SxK-TMDs). Symbols in brackets indicate the charges of $C$-terminal aas at $\mathrm{pH}=7.4$.

The general reason for higher fusion efficiencies of the E3/K3-LR/TMD peptide hybrids compared to PNA-LR/TMD peptide hybrids are the recognition motifs. E3/K3 coiled coils bring the LRs/TMDs of Syb2 and Sx1A in closer contact than the PNA1/PNA3 duplexes (Figures 2.6.2.1-1 and 2.6.2.2-1). Therefore, the mechanical 
force exerted on membranes and accompanied perturbations caused by TMD immersion are stronger for E3/K3 based model peptides. Moreover, the thermal stability for E3/K3 coiled coils $\left(T_{\text {melt }}=57^{\circ} \mathrm{C}\right)$ is higher than for PNA1/PNA3 duplexes ( $T_{\text {melt }}=46^{\circ} \mathrm{C}$ ) and thus, higher amounts of energy are released during coiled-coil formation. This might enhance the process of membrane fusion. ${ }^{[15,164]}$ Higher inner leaflet mixing rates due to full fusion underlines the stronger fusion efficiency of E3/K3-LR/TMD peptide hybrids (Figure 3.1-2, A-B).

A

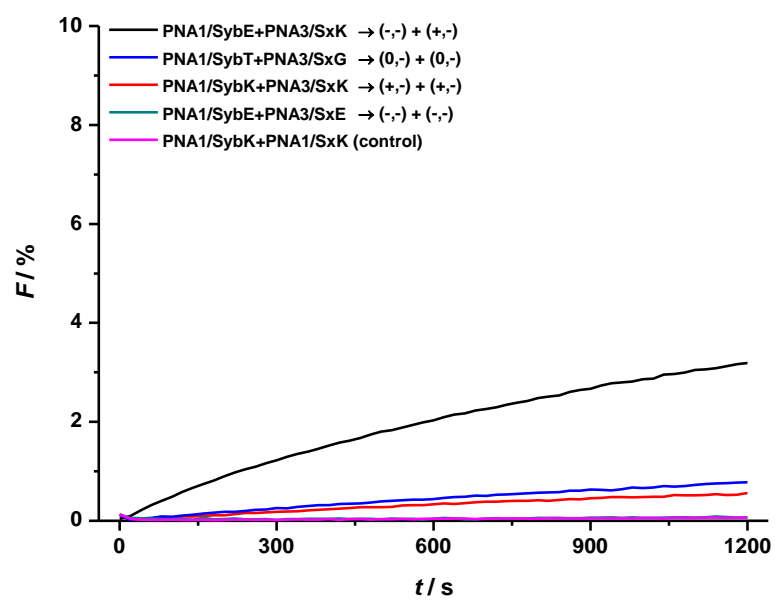

B

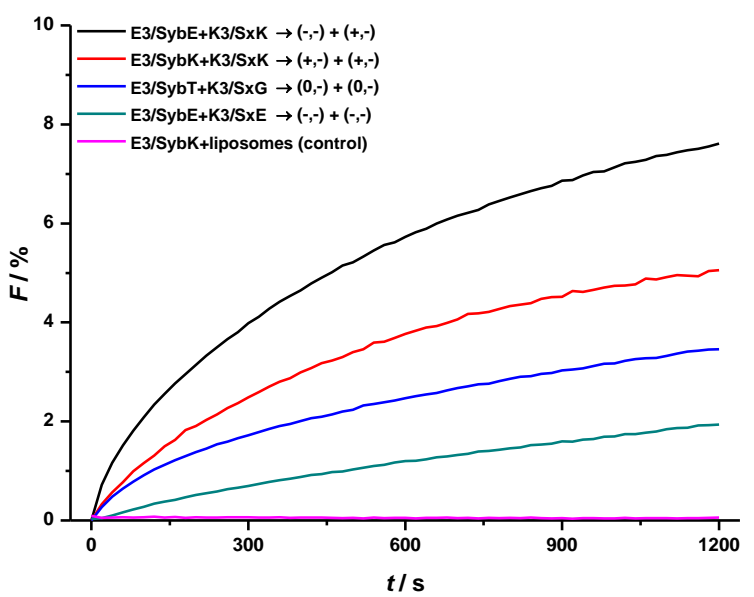

Figure 3.1-2 Inner leaflet mixing of liposomes decorated with SNARE mimetics carrying carboxylic acid termini (SybX- and SxY-TMDs). The increase of donor emission (NBD) occurs due to dilution of labelled lipids of the inner leaflets. Emission intensities are normalised by using the $100 \%$ intensity value after Triton $X$ treatment with fluorescent NBD in outer and inner leaflets (same $100 \%$ value as for the normalisation of TLM measurements): Parallel oriented recognition of A) PNA1/PNA3 duplex and B) E3/K3 coiled-coil formation. Native $C$-terminal threonine of the SybT-TMD and glycine of the SxG-TMD were exchanged either by lysines (SybK- and SxK-TMDs), by glutamates (SybE- and SxE-TMDs) or by glutamates for the SybT-TMD and lysines for the SxG-TMD (SybE- and SxK-TMDs). Symbols in brackets indicate charges of $C$-terminal aas at $\mathrm{pH}=7.4$.

During ILM measurements of proteoliposomes with PNA based model peptides, only the combination PNA1/SybE + PNA3/SxK exhibits a significant increase of NBD emission ( 3\%) indicative for the merger of inner leaflets. For the E3/K3-LR/TMD peptide hybrids, all $C$-terminally modified peptides exhibit certain degrees of inner leaflet mixing. This observation supports the assumption that $C$-terminal zippering is especially important for the transition of hemifusion intermediates (stalk and/or diaphragm intermediates, Figure 2.7-1) to full fusion. ${ }^{[14]} \mathrm{E} 3 / \mathrm{K} 3$ recognition corresponds rather to native 
SNARE complex formation than PNA1/PNA3 interactions, because the twists of the E3/K3-coiled coil and the Syb2/Sx1A-coil coincide with 2.9\%/aa. In the case of a PNA helix, the twist per bp is $20^{\circ}$. This difference might result in an unfavourable positioning of the $\mathrm{N}$-terminal aas of the LRs of Syb2 and Sx1A for interactions which might be important for $C$-terminal zippering. ${ }^{[10]}$ However, the distance of $1.4 \mathrm{~nm}$ between the facing helices in the $C$-terminal region of the E3/K3-coiled coil corresponds to the diameter of an aeg-PNA helix. The distance in the $C$-terminal region of the Syb2/Sx1A-coil is with $0.6 \mathrm{~nm}$ much smaller. Concerning these structural features both E3/K3 and PNA based recognition are inappropriate to mimic native SNARE recognition (Sections 2.6.2.1 and 2.6.2.2). Furthermore, aas allocated to the coiled-coil formation of the native SNARE motif reach into the LRs of Syb2 and Sx1A.[127] However, this does not correspond to the coiled-coil register of the used E3/K3 model peptides (Appendix, Comparison of Native and Artificial Sequences). Consequences of these mismatches are discussed later (Section 3.4.6.2). 


\subsection{1) Recognition Orientation of PNA-LR/TMD Peptide Hy- brids}

Parallel interactions of SNARE mimetics with PNA recognition units lead mainly to stronger leaflet mixing than those with antiparallel recognition (Figures 3.1.1-1 and 3.1.1-3). This observation is in line with the prevailing parallel mode of recognition of native SNARE proteins and underlines the assumption that parallel interacting model peptides can exert more force on the membranes during the process of recognition via their TMDs (Figure 2.7-2). ${ }^{[3]}$

A

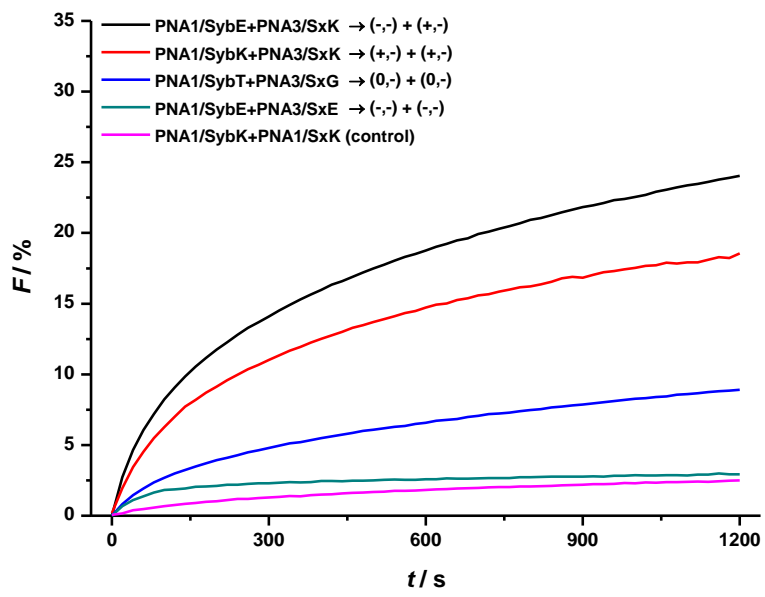

C

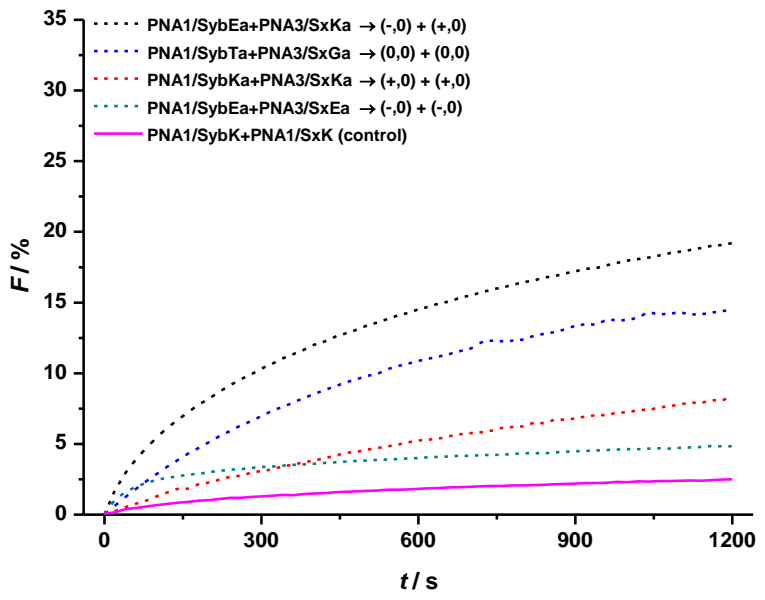

B

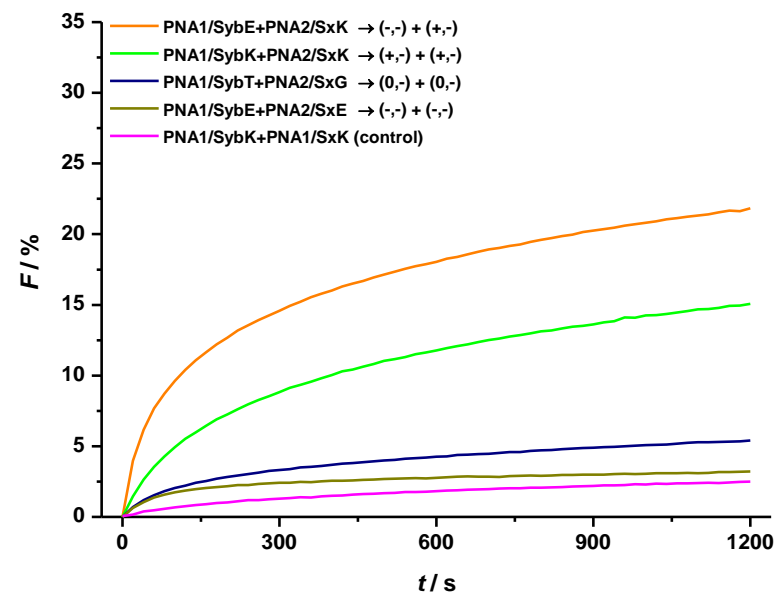

D

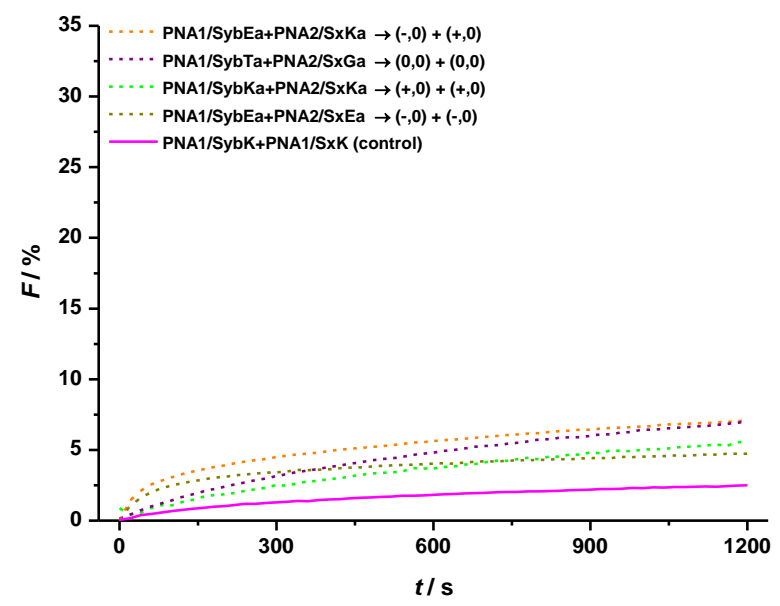

Figure 3.1.1-1 Total leaflet mixing of liposomes decorated with SNARE mimetics carrying carboxylic acid (SybX- and SxY-TMDs, A-B) and amide termini (SybXa-and SxYa-TMDs, C-D). The increase of donor emission (NBD) occurs due to the mixing of lipids of the outer and inner leaflets. Peptide combinations presented in $\mathbf{A}$ ) and $\mathbf{C}$ ) follow the parallel recognition mode. Combinations in $\mathbf{B}$ ) and $\mathbf{D}$ ) follow the antiparallel recognition mode. Native $C$-terminal threonines of the SybT(a)-TMDs and glycines of the 
SxG(a)-TMDs were exchanged either by lysines (SybK(a)- and SxK(a)-TMDs), by glutamates (SybE(a)and SxE(a)-TMDs) or by glutamates for the SybT(a)-TMDs and lysines for the SxG(a)-TMDs (SybE(a)- and SxK(a)-TMDs). Symbols in brackets indicate charges of $C$-terminal aas at $\mathrm{pH}=7.4$.

Interestingly, the drop of fusion efficiency in TLM assays due to antiparallel recognition is more distinct for PNA-LR/TMD peptide hybrids with $C$-terminal amides (Figure 3.1.1-1, C-D) than for model peptides with carboxylic acids (Figure 3.1.1-1, A-B). Amide like $C$-termini are less charged and therefore their immersion into the membranes during parallel recognition and accompanied membrane perturbation is favoured due to a lower energy barrier for TMD motions compared to $C$-terminal carboxylic acids, which are stronger charged (Section 3.2). If model peptides interact in the antiparallel mode, immersion of the $C$-termini into the membranes is not possible due to an inappropriate arrangement of the TMDs (Figure 2.7-2, A-B). In conclusion, less membrane perturbation through the $C$-termini during antiparallel recognition has stronger effects on leaflet mixing of liposomes with model peptides carrying $C$-terminal amides than on liposomes with peptides carrying carboxylic acids, because the membrane perturbation is less distinct for stronger charged $C$-termini during parallel recognition. Nevertheless, TLM measurements using antiparallel interacting model peptides with $C$-terminal carboxylic acids and amides - even when slight - was detected (Figure 3.1.1-1, $B$ and D). Thus, the antiparallel recognition of PNA-LR/TMD peptide hybrids probably act in form of peptidic bridges between the membranes bringing them in spatial proximity. ${ }^{[165]}$ The length of the antiparallel PNA duplex with $0.32 \mathrm{~nm}$ rise/bp and the LRs of Syb2 and Sx1A as stretched $\alpha$-helices with $0.15 \mathrm{~nm}$ rise/aa is estimated to be $\sim 6 \mathrm{~nm} .{ }^{[113]}$ At a distance of $6 \mathrm{~nm}$, no membrane fusion is conceivable. Therefore, other reasons might play a role. Leaflet mixing induced by antiparallel interacting PNA-LR/TMD peptide hybrids with $C$-terminal carboxylic acids is weak except for the combinations PNA1/SybE + PNA2/SxK and PNA1/SybK + PNA2/SxK which exhibit strong TLM (Figure 3.1.1-1, B). The $C$-terminal lysines of these model peptide combinations might enhance membrane perturbation through electrostatic interactions of the positively charged amino groups of the side chains with the headgroups of the phospholipids and hydrophobic interactions of the residual hydrocarbon chains with the lipid tails (snorkelling interactions). ${ }^{[166]}$ The combination of peptidic connection and membrane perturbation through $C$-terminal lysines seem to be sufficient to induce TLM. For the combination PNA1/SybE + PNA2/SxK, lysines support leaflet mixing only through 
the lysine modified SxTMDs. However, the combinations of PNA1/SybE + PNA3/SxK (parallel) and PNA1/SybE + PNA2/SxK (antiparallel) exhibit similar extents of leaflet mixing (Figure 3.1.1-2). The increase in NBD emission is even higher in the first $450 \mathrm{~s}$ of the measurement of antiparallel recognition. Furthermore, $C$-terminal lysines as amides should have similar perturbing effects like lysines as carboxylic acids, but for the combination PNA1/SybKa + PNA2/SxKa (Figure 3.1.1-1, D) only an emission increase of $\sim 5 \%$ (corresponding carboxylic acid combination: $13 \%$ ) is detected. These contradictory results are rather explainable by the instability of proteoliposomes, differences in added amounts of liposomes during measurements and/or by differences in the degree of peptide insertion than by $C$-terminal modifications and the mode of recognition. These aspects are discussed in more detail in Sections 3.4.2, 3.4.3 and 3.4.4.

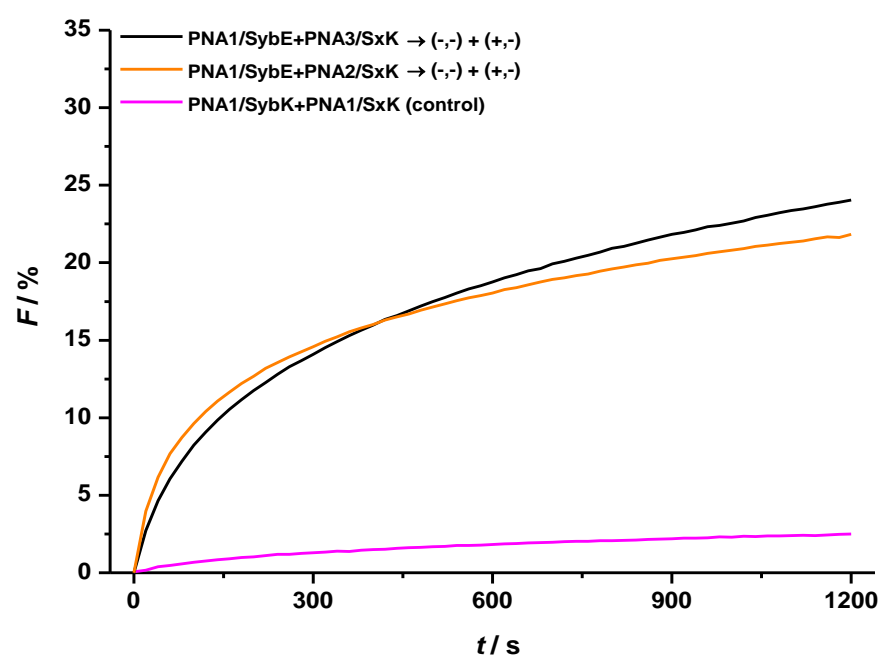

Figure 3.1.1-2 Total leaflet mixing of liposome populations with following peptide combinations: PNA1/SybE + PNA3/SxK (parallel recognition, black graph), PNA1/SybE + PNA2/SxK (antiparallel recognition, orange graph) and PNA1/SybK + PNA1/SxK (control, magenta graph). Symbols in brackets indicate charges of $C$-terminal aas at $\mathrm{pH}=7.4$.

ILM assays for the antiparallel recognition between SNARE mimetics with $C$-terminal carboxylic acids have mainly the same tendencies compared to the results of TLM assays, i.e. combinations PNA1/SybT + PNA2/SxG and PNA1/SybE + PNA2/SxE exhibit only slight hemifusion because of slight TLM rates (<5\%, Figure 3.1.1-1, B) and no significant ILM rates (Figure 3.1.1-3, B). However, concerning the combination PNA1/SybE + PNA2/SxK significant leaflet mixing was also observed in ILM assays (Figure 3.1.1-3, B). The observation of leaflet mixing in TLM and ILM assays indicates full fusion. Strong TLM is usually accompanied by relative strong ILM. One exception 
herein is the combination PNA1/SybK + PNA2/SxK. For this mixture, an increase in NBD emission of $\sim 15 \%$ during TLM assays was detected (Figure 3.1.1-1, B), but in ILM assays no significant increase was observed (Figure 3.1.1-3, B). Thus, lysine modified PNA-LR/TMD peptide hybrids induce only strong hemifusion, if they interact in antiparallel orientation. SNARE mimetics with $C$-terminal amides show only a slight increase in NBD emission for both TLM $(<10 \%$, Figure 3.1.1-1, D) and ILM $(<1 \%$, Figure 3.1.1-3, D).

A

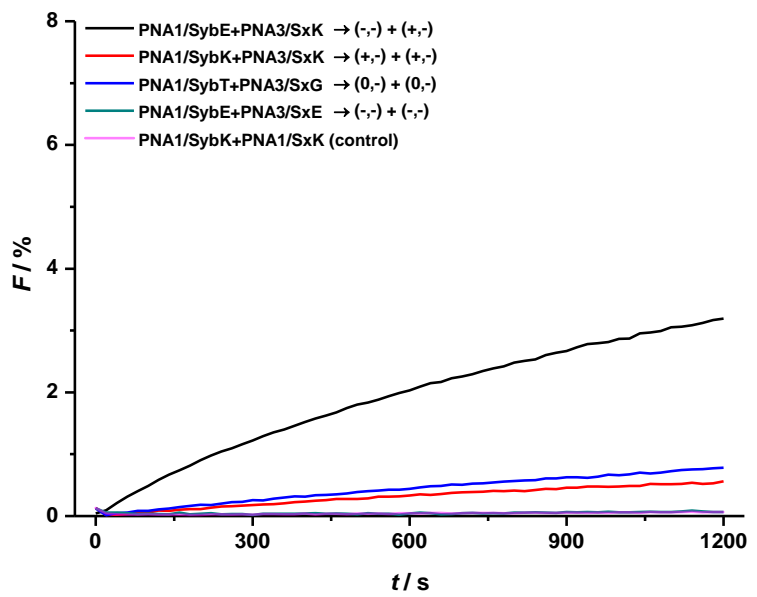

C

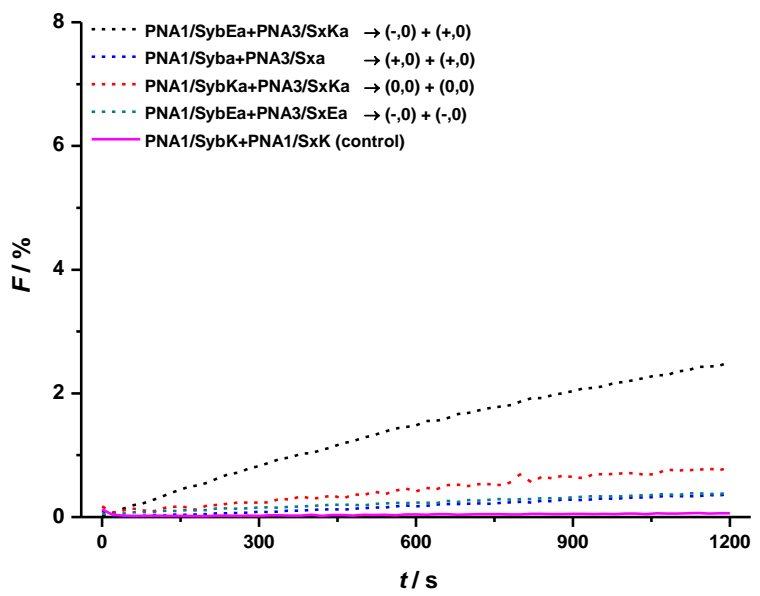

B

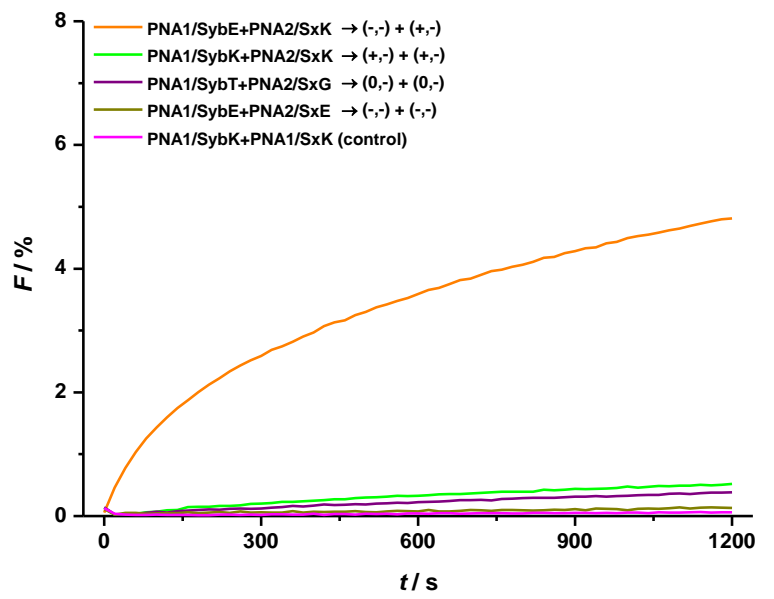

D

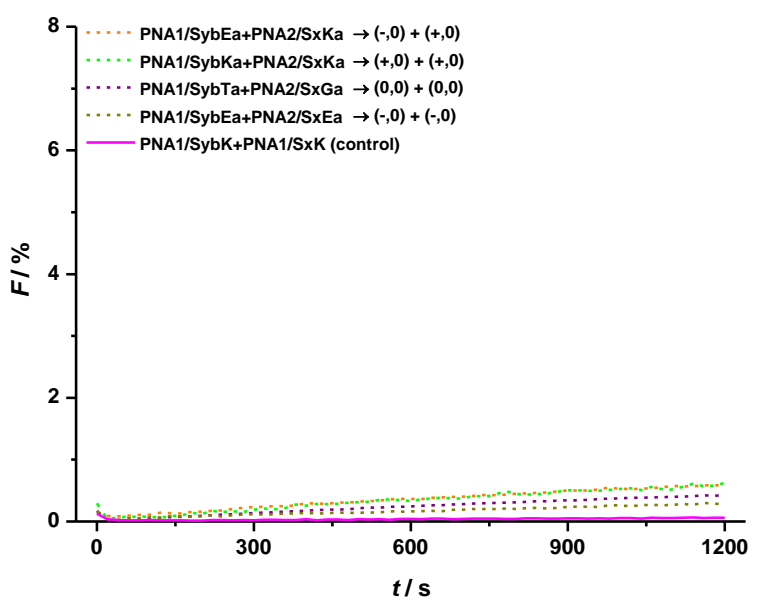

Figure 3.1.1-3 Inner leaflet mixing of PNA-LR/TMD peptide hybrids with carboxylic acid (SybX- and SXY-TMDs) and amide termini (SybXa- and SxYa-TMDs). The increase of donor emission (NBD) occurs due to mixing of the inner leaflets. Emission intensities are normalised by using the $100 \%$ intensity value after Triton $X$ treatment with fluorescent NBD in outer and inner leaflet (the same $100 \%$ value as for the normalisation of the TLM assays). A) and C) Parallel interacting PNA1 and PNA3 recognition motifs. B) and D) PNA1 and PNA2 sequences interact in antiparallel orientation ( $\mathrm{N}$ - and $\mathrm{C}$-termini are at different sides of the duplex). 
Consequently, antiparallel recognition between peptides with $C$-terminal amides lead only to weak hemifusion. Although it was expected that at least the combinations with $C$-terminal lysines as amides would induce stronger membrane fusion. Like mentioned for TLM assays, these contradictory results might be rather explainable by experimental aspects like proteoliposome instability, added amounts of proteoliposomes for the leaflet mixing assays and/or by different degrees of peptide insertion than by structural features of the model peptides. For ILM assays, the same contradictory results in regard to parallel and antiparallel recognition between SybE- and SxK-TMDs were obtained (Figure 3.1.1-4). All other combinations of the PNA-LR/TMD peptide hybrids in parallel orientation were superior compared to antiparallel oriented hybrids concerning inner leaflet mixing assays. The reason for it might not be found in the mode of recognition but again rather in experimental uncertainties. For instance, it is conceivable that the loss of lipid material during liposome preparation was higher for liposomes decorated with PNA3/SxK than for liposomes carrying PNA2/SxK (Section 3.4.3).

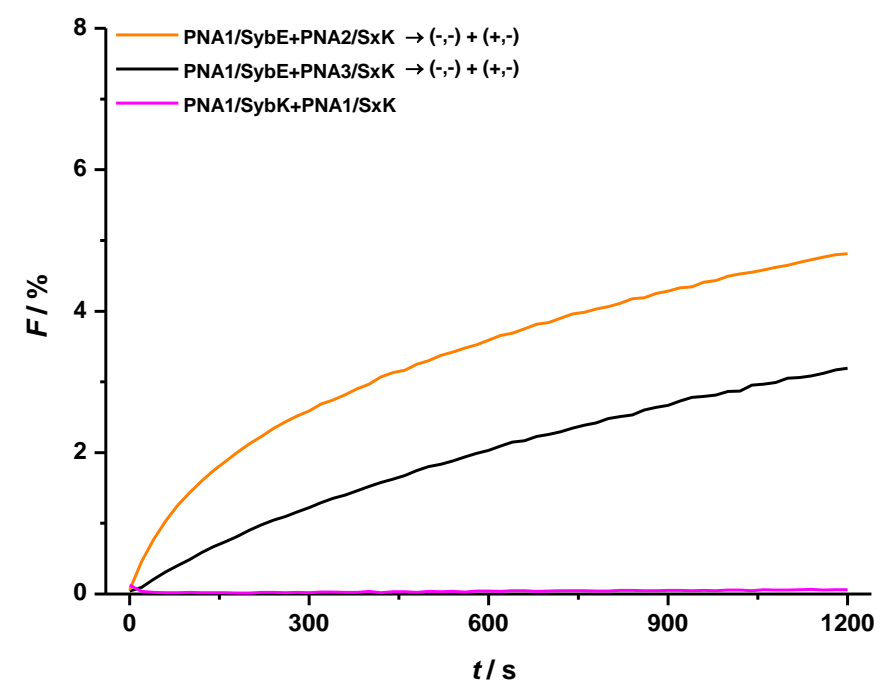

Figure 3.1.1-4 Inner leaflet mixing of liposome populations with following peptide combinations: PNA1/SybE + PNA3/SxK (parallel recognition, black graph), PNA1/SybE + PNA2/SxK (antiparallel recognition, orange graph) and PNA1/SybK + PNA1/SxK (magenta graph). 


\subsection{2) Recognition Orientation of E3/K3-LR/TMD Peptide Hybrids}

For the induction of antiparallel recognition, the E3 sequence was inverted yielding the iE3 sequence (Figure 2.6.2.2-1, E). However, through the sequence inversion the hydrophobic core of the coiled coil is altered. Polar glutamate and alanine at positions a and $\boldsymbol{d}$ change knob into hole $(\mathrm{KIH})$-interactions dramatically. Another state of oligomerisation is required to ensure preferred hydrophobic packing. ZHENG et al. demonstrated that the inversion of the E3 sequence changes the orientation and oligomerisation of the coiled coil from a parallel dimeric to an antiparallel tetrameric assembly. ${ }^{[164]}$ In this study, paramagnetic NMR spectroscopy, FRET based measurements and coarse-grained molecular dynamics (CGMD) simulations demonstrated that assemblies of iE3 and K3 peptides (without the LRs/TMDs of Syb2 and Sx1A) are antiparallel oriented.

A

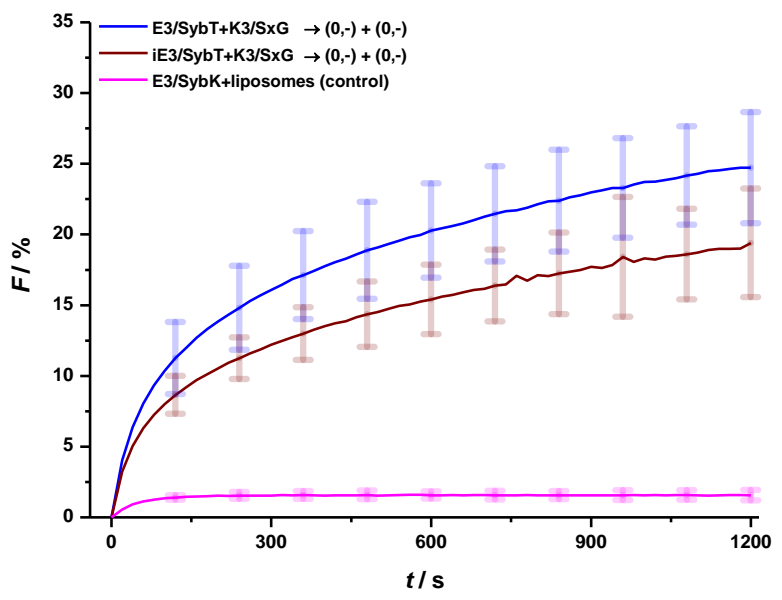

C

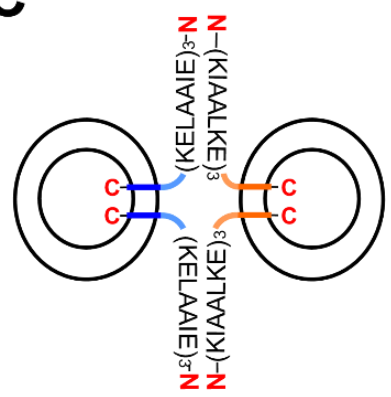

B
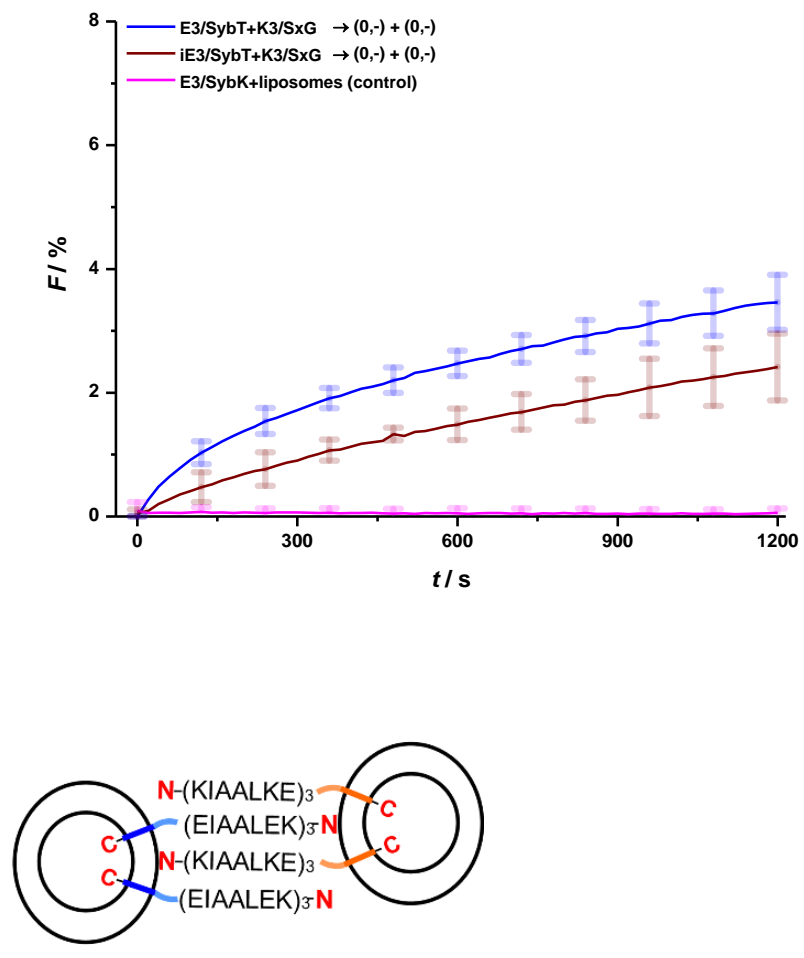

Figure 3.1.2-1 Comparison of parallel and antiparallel coiled-coil forming SNARE mimetics: A) TLM. B) ILM. C) Illustration of the parallel (left) and the antiparallel (right) recognition mode of the (i)E3/K3-LR/TMD peptide hybrids. For parallel recognition, one SybTMD (dark blue) and one SxTMD 
(dark orange) per coiled coil are involved, respectively (dimer formation). For antiparallel recognition, two SybTMDs and two SxTMDs participate per coiled coil, respectively (tetramer formation).

A Job-plot indicates a 1:1 binding stoichiometry and analytical ultracentrifugation as well as CGMD simulations suggest tetrameric coiled coils with two iE3 and K3 peptides, respectively. Within the tetramer $\mathrm{IE} 3$ and $\mathrm{K} 3$ peptides are antiparallel to each other, whereby identical peptides are parallel oriented. This change in oligomerisation must be considered, when parallel and antiparallel recognition of coiled-coil SNARE mimetics are compared (Figure 3.1.2-1, C). TLM and ILM assays demonstrated that the parallel and antiparallel recognition between the SNARE mimetics with native $C$-terminal ends induce leaflet mixing (hemifusion and partial full fusion). Parallel interactions led to slightly stronger leaflet mixing (Figure 3.1.2-1, A-B). This is an agreement with native SNARE proteins which follow mainly the parallel recognition mode. Compared to PNA induced antiparallel recognition between SybTMDs and SxTMDs with native $C$-terminal aas (PNA1/SybT + PNA2/SxG, $\sim 5 \%$ emission increase, Figure 3.1.1-1, B), the antiparallel tetrameric coiled coil evokes much stronger TLM (iE3/SybT + K3/SxG, 17\% emission increase, Figure 3.1.2-1, A). ILM assays confirm TLM assays with an increase of emission of $\sim 2 \%$ (Figure 3.1.2-1, B). For the cognate PNA-LR/TMD peptide hybrids, no ILM was detected (Figure 3.1.1-3, B). In this case, the stronger increase is not explainable by $C$-terminal zippering, because these interactions are not possible for $\mathrm{iE} 3 / \mathrm{K} 3$ recognition. Antiparallel tetramer formation connects the apposed membranes. The length of the tetramer (21 aas) and the LRs of Syb2 and Sx1A (10 aas, respectively) is estimated to be $\sim 6 \mathrm{~nm}$, if one assumes stretched $\alpha$-helices with a rise of $0.15 \mathrm{~nm}$ per aa. However, a distance of $6 \mathrm{~nm}$ is not sufficient to induce membrane fusion. It is supposed that K3 peptides interact with the headgroups of phospholipids and thereby shaping the membranes. This results in positively curved protrusions. In addition, DOPE (negative curvature supporting stalk formation) and cholesterol (stabilisation of the fusion pore) accumulate in the vicinity of K3. ${ }^{[52]}$ Furthermore, $\mathrm{K} 3$ binds not only to the membrane where it is inserted via its SxTMD, but also to the opposing membrane. ${ }^{[167]}$ These peptide-membrane interactions facilitate the fusion of docked membranes. For aeg-PNA, no membrane interactions are reported. Membrane activity of K3 is logically also crucial for parallel coiled-coil mediated membrane fusion. The $\mathrm{K} 3$ based model peptides - although initially not expected and desired - do not only mimic the SNARE motif of Sx1A, but also 
membrane shaping activities which are known to be involved in natural fusion processes (Section 2.4.2). However, the exact extent of fusion supporting interactions between $\mathrm{K} 3$ and the membranes is not known.

\section{2) Influence of $C$-Terminal Carboxylic Acids and Am- ides of SNARE Mimetics on Leaflet Mixing}

The tendency of higher fusion efficiency for E3/K3-LR/TMD peptide hybrids compared to PNA based model peptides with $C$-terminal carboxylic acids is also observed for the SNARE mimetics with amides as $C$-termini. However, the differences in TLM are even higher than for carboxylic acid-like $C$-termini (Figure 3.2-1, A-B and C-D). This difference is explainable by the release of more energy during $E 3 / K 3$ recognition than during PNA1/PNA3 interactions. Furthermore, E3/K3-LR/TMD peptide hybrids mimic native SNARE complex formation better concerning the transition between recognition units and LRs (Sections 2.6.2.2 and 3.1). Therefore, E3/K3-LR/TMD peptide hybrids exert probably more force on membranes through its $C$-termini during recognition than PNA-LR/TMD peptide hybrids. In the case of $C$-terminal amides, the negative charges of the carboxylic acid groups are missing. Therefore, the energy-barrier for the transition of the amide $C$-termini into the hydrophobic core of the membrane is lower than for the carboxylic acid $C$-termini. Membrane fusion supported by the perturbation of lipid continuity through the immersion of $C$-termini is thought to be more efficient for $C$-terminal amides. ${ }^{[14,17]}$ Increased fusion efficiencies of $C$-terminal amides were observed for all combinations in the group of E3/K3 based model peptides (compare $B$ and D, Figure 3.2-1). 
A

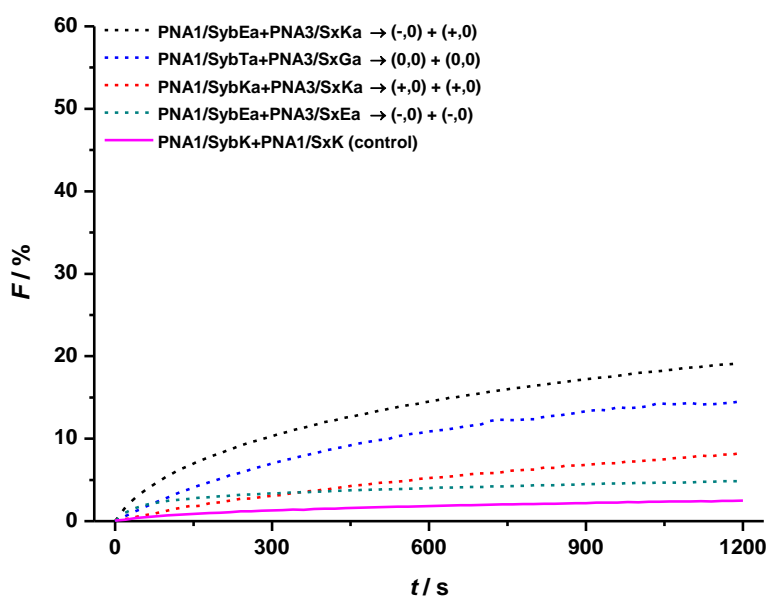

C

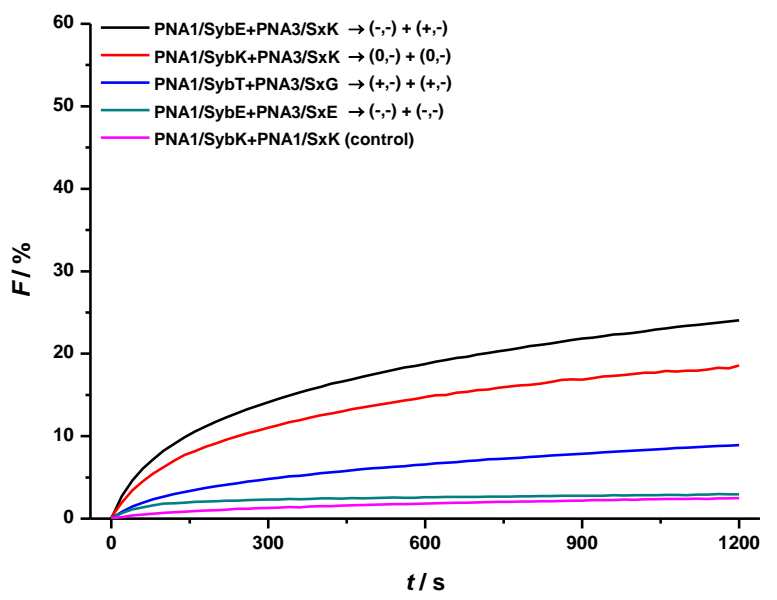

B

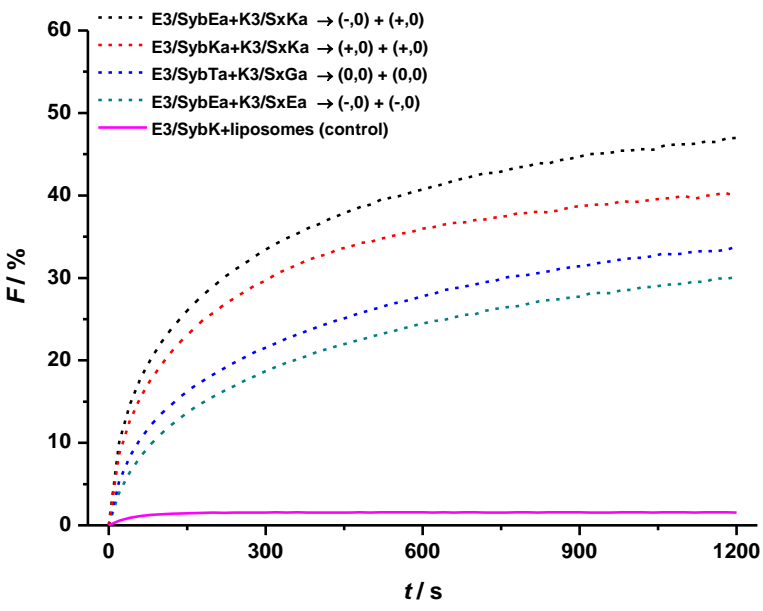

D

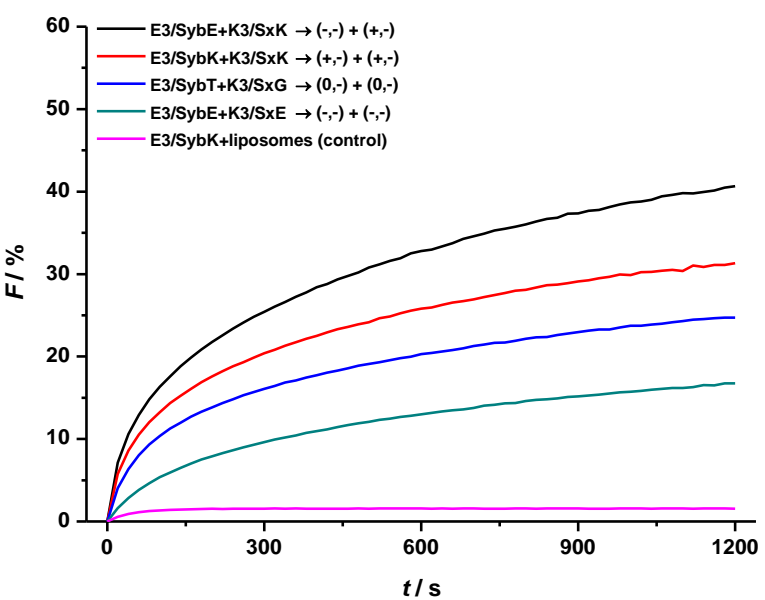

Figure 3.2-1 Total leaflet mixing of liposomes decorated with SNARE mimetics carrying amide (SybXaand SxYa-TMDs, A-B) and carboxylic acid termini (SybX-and SxY-TMDs, C-D). Increase of donor emission (NBD) occurs due to labelled lipids of the outer and inner leaflets. All combinations follow the parallel recognition mode. The exchange of aas for peptides with amide termini is the same as for peptides with carboxylic acid termini.

Within the PNA based peptide group, only combinations of natural aas and glutamate as $C$-terminal amides (PNA1/SybTa + PNA3/SxGa and PNA1/SybEa + PNA3/SxEa) fuse with higher efficiency than the same combinations as carboxylic acids (PNA1/SybT + PNA3/SxG and PNA1/SybE + PNA3/SxE) (Figure 3.2-2, A). However, in the case of PNA-LR/TMD peptide hybrids with lysines or glutamates and lysines, carboxylic acids (PNA1/SybK + PNA3/SxK and PNA1/SybE + PNA3/SxK) fuse more efficiently than amides (PNA1/SybKa + PNA3/SxKa and PNA1/SybEa + PNA3/SxKa) (Figure 3.2-2, B). 
A

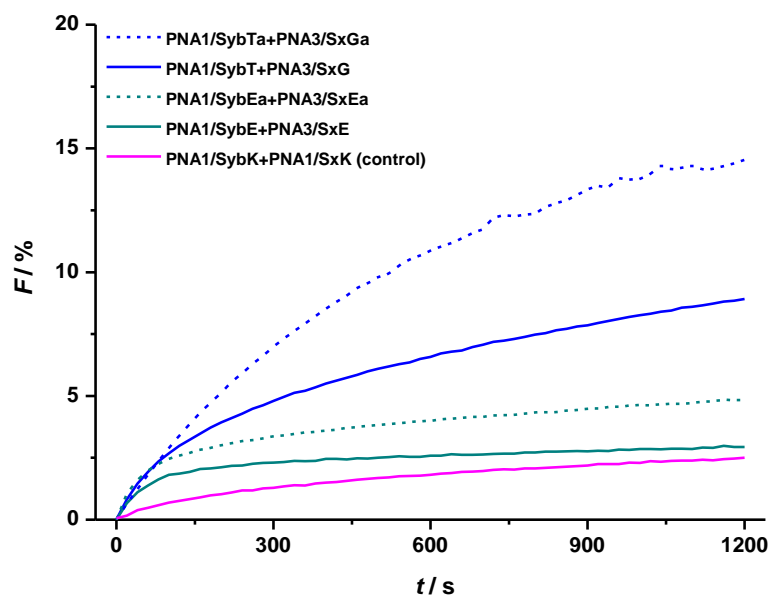

B

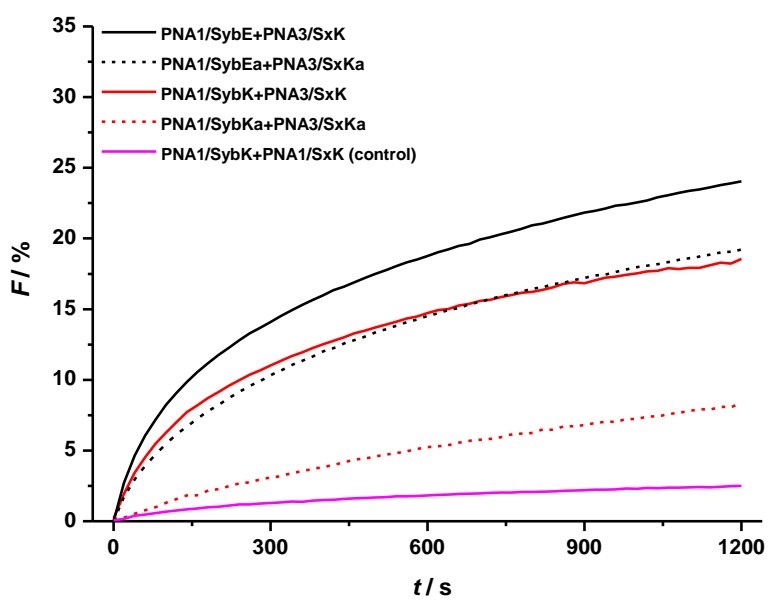

Figure 3.2-2 Selections of PNA-LR/TMD peptide hybrids which differ in TLM efficiency due to its $C$-termini: A) Peptides with amide $C$-termini exhibit stronger fusion than those with $C$-terminal carboxylic acids. B) Peptides with carboxylic acid $C$-termini show stronger fusion than those with amide $C$-termini.

This is contradictory, because the charge is also reduced in the case of $C$-terminal lysines for the SybTMD and SxTMD or glutamates for the SybTMD and lysines for the SxTMD as amides. Different degrees of peptide insertion during liposome preparation might again be one reason for the above mentioned contrary fusion behaviour (Section 3.4.4). Another reason for stronger leaflet mixing of the combinations PNA1/SybE + PNA3/SxK and PNA1/SybK + PNA3/SxK compared to the same combinations with $C$-terminal amides might be the low stability of proteoliposomes carrying PNA-LR/TMD peptide hybrids with $C$-terminal lysines. Both as carboxylic acids and as amides, these peptides seem to destabilise membranes stronger than peptides with native aas or glutamates. For instance, for all PNA based model peptides with $C$-terminal lysines, visible aggregation processes were observed within 30-60 min after liposome preparation (Tables 3.4.2-2 and 3.4.2-3). Native aas or glutamates induced sometimes fast aggregation as well, especially as amides, but with less propensity. In general, proteoliposomes with PNA1/SybEa and PNA1/SybKa are less stable than those with the cognate SNARE mimetics PNA1/SybE and PNA1/SybK. The instability of proeteoliposomes might be another reason for the contradictory results mentioned above, because the detection of leaflet mixing is influenced by the accompanied aggregation processes (Section 3.4.2). 


\section{3) Significant Differences in Leaflet Mixing Due to C-Terminal Modifications}

It is supposed that the charges of the $C$-termini of Syb2 and Sx1A are crucial for the transition of hemifusion intermediates to full fusion because of $C$-terminal zippering accompanied immersion of the $C$-terminal ends into the membranes. The caused discontinuity might lead to a rearrangement of lipids which results in fusion pores. ${ }^{[14,17]}$ The application of modified SNARE mimetics in leaflet mixing assays should help to clarify this assumption. All proteoliposome combinations were measured three times. However, the individual measurements differ partially considerable from each other concerning the detected increases of NBD emission. These variations are explicable by experimental uncertainties (Section 3.4). In the following sections, only significant differences between total leaflet mixing measurements with regard to $C$-terminal modifications of the SNARE mimetics are discussed.

\subsection{1) PNA-LR/TMD Peptide Hybrids with $C$-Terminal Car- boxylic Acids}

For parallel PNA recognition, the combination PNA1/SybE + PNA3/SxK (fluorescence increase of $\sim 24 \%$ after 20 min detection time) can be clearly distinguished from the combinations PNA1/SybT + PNA3/SxG ( 8\%) and PNA1/SybE + PNA3/SxE ( 3\%) in TLM assays (Figure 3.3.1-1, A). However, a clear distinction between ILM curves is not possible (Figure 3.3.1-1, B). C-terminal threonine and glycine for the SybTMD and SxTMD (PNA1/SybT + PNA3/SxG), respectively, represent the native $C$-terminal aas and effective fusion was expected. However, only slight hemifusion was observed for this combination, because of the relative slight fluorescence increase in TLM assays ( 8\%, Figure 3.3.1-1, A) and negligible low increase in ILM assays $(<1 \%$, Figure 3.3.1-1, B). 
A

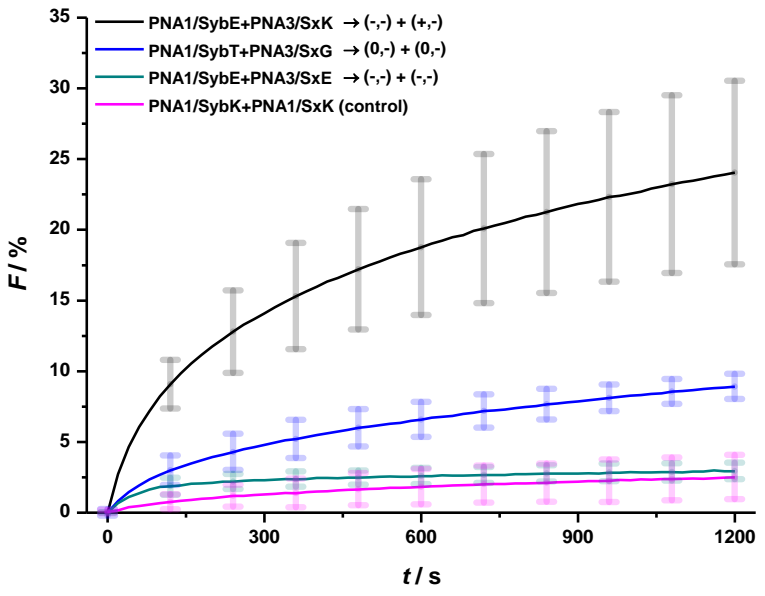

B

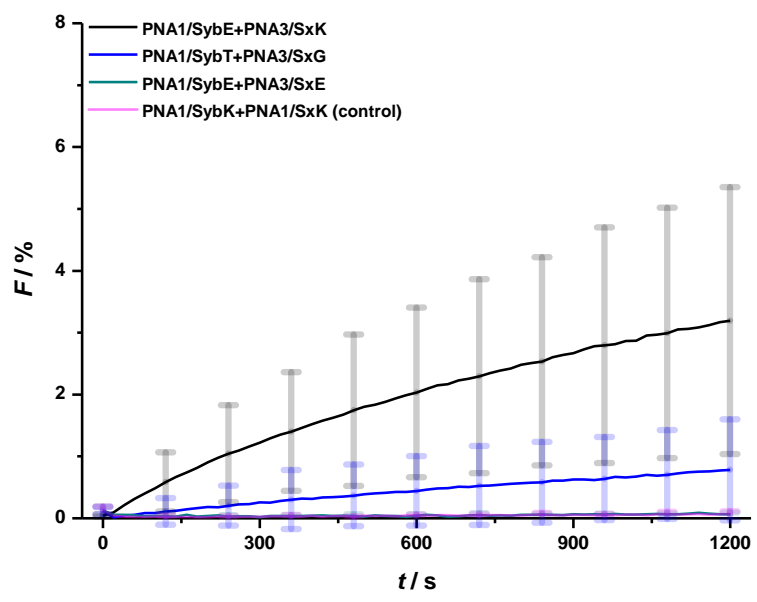

Figure 3.3.1-1 Leaflet mixing measurements with (significant) differences in the increase of NBD fluorescence: A) TLM and B) ILM results of parallel interacting PNA-LR/TMD peptide hybrids with $C$-terminal glutamates and threonines for the SybTMDs as well as lysines, glycines and glutamates for SxTMDs (all $C$-terminal aas as carboxylic acids).

Parallel PNA recognition is not sufficient to exert enough force on the TMDs to rearrange lipids for full fusion by immersing $C$-terminal ends because of the relative high energy barriers for the transition of negative charges of the carboxylic acid groups of SybT- and SxG-TMDs from the water/membrane interface into the headgroup region/hydrophobic core of the membranes. Furthermore, additional energy through $C$-terminal zippering of the LRs/TMDs of Syb2 and Sx1A is unlikely due to topological differences between the PNA duplexes and LRs/TMDs of the SNARE mimetics (Sections 2.6.2.1 and 3.5.2). C-terminal glutamates for the SybTMDs and SxTMDs (PNA1/SybE + PNA3/SxE) hinder efficient fusion more distinctly. During TLM a fluorescence increase of $<3 \%$ within 20 min was detected which is in the range of the control experiment (Figure 3.3.1-1, A). This weak fluorescence increase might indicate docking with a slight unspecific exchange of lipids of the outer leaflets of the PNA1/SybE and PNA3/SxE liposomes, but lipid mixing of the inner leaflets is excluded, because ILM assays exhibit no fluorescence increase at all (Figure 3.3.1-1, B). The double negative charge of the $C$-terminal glutamates raises the energy barrier for the transition of $C$-termini into the membranes (Section 3.5.2). Therefore, no rearrangement of the lipids through TMD immersion and membrane fusion occur. ${ }^{[14,17]}$ Intriguingly, the combination of glutamates for the SybTMDs and lysines for the SxTMDs (PNA1/SybE + PNA3/SxK) exhibit the strongest leaflet mixing rates (24\% for TLM and 
$3 \%$ for ILM, Figure 3.3.1-1) of PNA based model peptides with $C$-terminal carboxylic acids in the parallel recognition mode. This is partially contradictory, because glutamate modifications of the SybTMDs have hindering effects on fusion as well. However, $C$-terminal lysines of SxTMDs have destabilising effects on the membrane continuity and support leaflet mixing. Nevertheless, $C$-terminal lysines for both SybTMD and SxTMD exhibit less leaflet mixing ( $18 \%$ ) than $C$-terminal glutamates and lysines (not significant), respectively (Figure 3.2-1, C). It is conceivable, that the low stability of the PNA1/SybK liposomes influence experimental outcomings because of aggregation processes which hamper the detection of membrane fusion. The combination PNA1/SybK + PNA3/SxK induces more efficient leaflet mixing ( 18\%) than the combinations of native $C$-termini ( $8 \%$ ) and $C$-terminal glutamates for both $C$-termini $(\sim 3 \%)$ with regard to TLM assays (Figure 3.3.1-2, A). However, no significant distinction regarding ILM assays was possible (Figure 3.3.1-2, B). Membrane perturbing effects of $C$-terminal zwitterionic lysines for the SybTMDs and SxTMDs support the fusion of PNA based SNARE mimetics more effectively than negative charges of the native TMDs or TMDs modified with glutamates. Leaflet mixing would probably be even stronger, if labelled PNA1/SybK liposomes were more stable, because the instability of labelled proteoliposomes affect the detection of membrane fusion by spectrofluorimetry (Section 3.4.2).

A

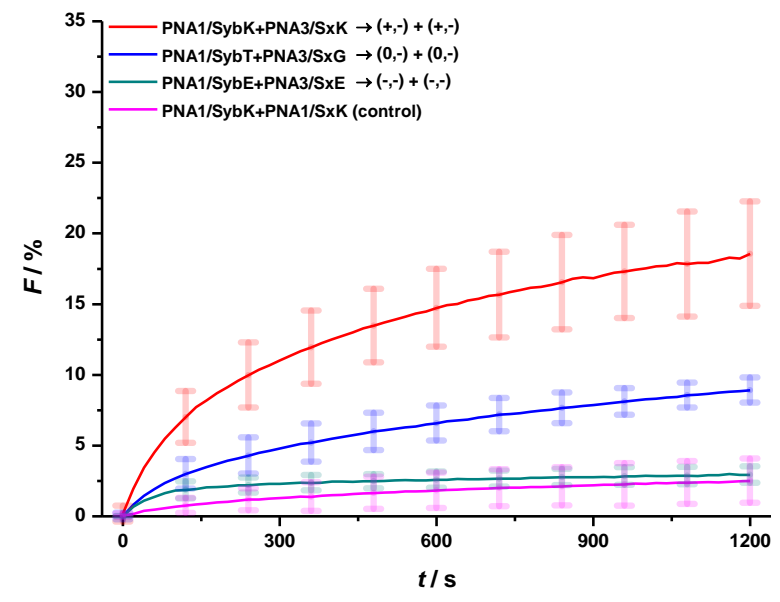

B

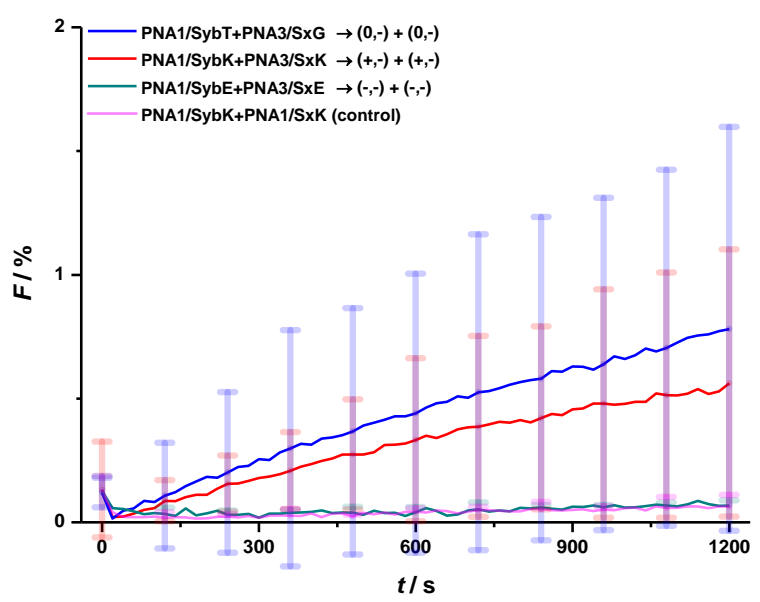

Figure 3.3.1-2 Total and inner leaflet mixing measurements with (significant) differences in the increase of NBD fluorescence: A) TLM and B) ILM results of parallel interacting PNA-LR/TMD peptide hybrids with $C$-terminal lysines, threonines and glutamates for the SybTMDs as well as lysines, glycines and glutamates for the SxTMDs (all $C$-terminal aas as carboxylic acids). 
Another fusion supporting feature of the lysine combination might be the supposed $C$-terminal attractions between the $C$-termini of SybK- and SxK-TMDs, respectively, due to opposing charges (Figure 2.7-2).[13] However, the antiparallel combination of lysine modified SNARE mimetics (PNA1/SybK + PNA2/SxK ( 15\%)) was also more efficient concerning membrane fusion (TLM) than the antiparallel recognition of native and glutamate modified TMDs (PNA1/SybT + PNA2/SxG ( 5\%) and $\mathrm{PNA} 1 / \mathrm{SybE}+\mathrm{PNA} 2 / \mathrm{SxE},(\sim 3 \%))$. If attractive forces between the $C$-termini of SNARE mimetics are important, it is expected that the lysine combination tends to less leaflet mixing in the antiparallel recognition mode, e.g. in the range of native TMDs, because no $C$-terminal interactions are conceivable due to the orientation of the TMDs to each other, but this was not the case. Membrane perturbing effects of $C$-terminal lysines support fusion during both parallel and antiparallel recognition. Thus, it is not possible to control the assumption of fusion supporting $C$-terminal attractions by the usage of $C$-terminal lysines. Rather, it is necessary to compare mixtures of SNARE mimetics with opposing $C$-terminal charges for one proteoliposome population, e.g. PNA1/SybKa and PNA1/SybEa for labelled liposomes as well as PNA3/SxKa and PNA3/SxEa (PNA2/SxKa and PNA2/SxEa) for unlabelled liposomes. Except of the lysine combination (PNA1/SybK + PNA2/SxK), fluorescence increases for antiparallel recognising model peptides with $C$-terminal carboxylic acids were always $<5 \%$ in TLM assays (Figure 3.3.1-3, A). These slight increases might be merely evoked by unspecific leaflet mixing due to spatial proximity induced by docking. All ILM assays of antiparallel interacting PNA-LR/TMD peptide hybrids were below $1 \%$ after 20 min of detection (Figure 3.3.1-3, B) and significant differentiations between the ILM measurements concerning $C$-terminal modifications are not possible. Thus, most antiparallel recognising PNA-LR/TMD peptide hybrids led to docking. For the antiparallel mode, only the lysine combination (PNA1/SybK + PNA2/SxK) induced hemifusion, because the fluorescence increase in TLM assays is high ( 15\%, Figure 3.3.1-3, A) and no significant emission increase concerning the ILM measurements was detected (Figure 3.3.1-3, B). Lysine induced membrane perturbations might support the transition from docked to hemifused proteoliposomes. 
A

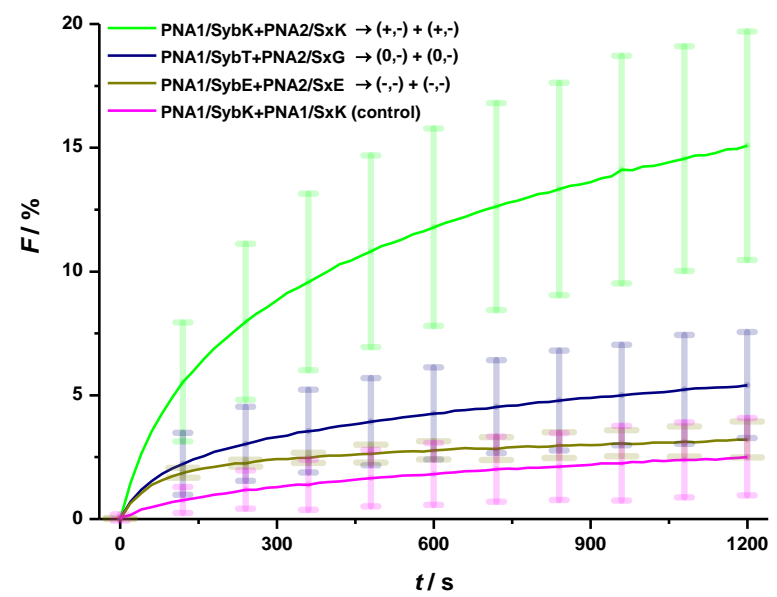

B

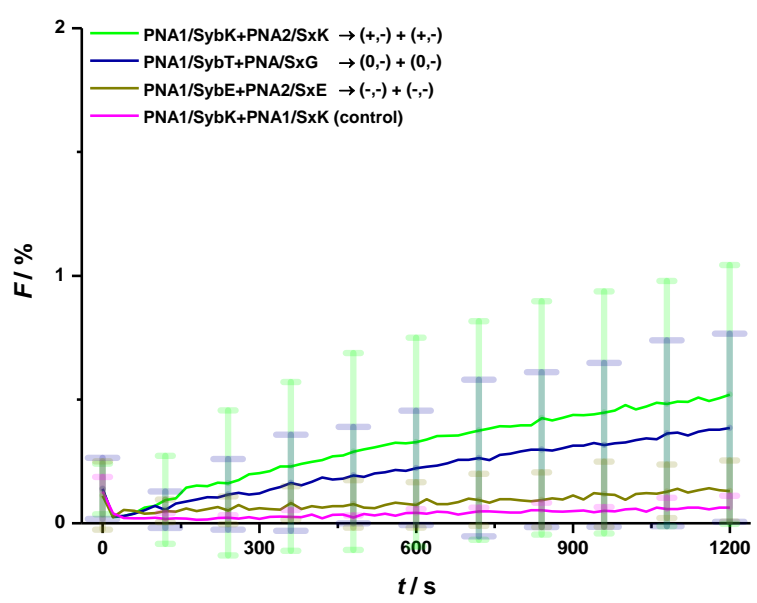

Figure 3.3.1-3 Total and inner leaflet mixing measurements with (significant) differences in the increase of NBD fluorescence: A) TLM and B) ILM results of antiparallel interacting PNA-LR/TMD peptide hybrids with $C$-terminal lysines, threonines and glutamates for the SybTMDs as well as lysines, glycines and glutamates for the SxTMDs (all $C$-terminal aas as carboxylic acids).

The higher leaflet mixing efficiency of the combination PNA1/SybE + PNA2/SxK compared to the combination PNA1/SybK + PNA2/SxK is contradictory, because no membrane perturbing effects of the SybE-TMDs are expected (Figure 3.3.1-4, A-B). The higher stability of PNA1/SybE liposomes compared to PNA1/SybK liposomes might be a reason for these results (Section 3.4.2). The fast aggregation of proteoliposomes induced by PNA1/SybK peptides might hamper molecular recognition between labelled and unlabelled proteoliposomes. Furthermore, fast aggregation of labelled PNA1/SybK liposomes led to high losses of lipid material during proteoliposome preparation for ILM assays (Section 3.4.1). The absence of a fluorescence increase for the combination PNA1/SybK + PNA2/SxK in ILM assays is probably a result of the loss of lipid material during size exclusion chromatography (Figure 3.3.1-4, B). 
A

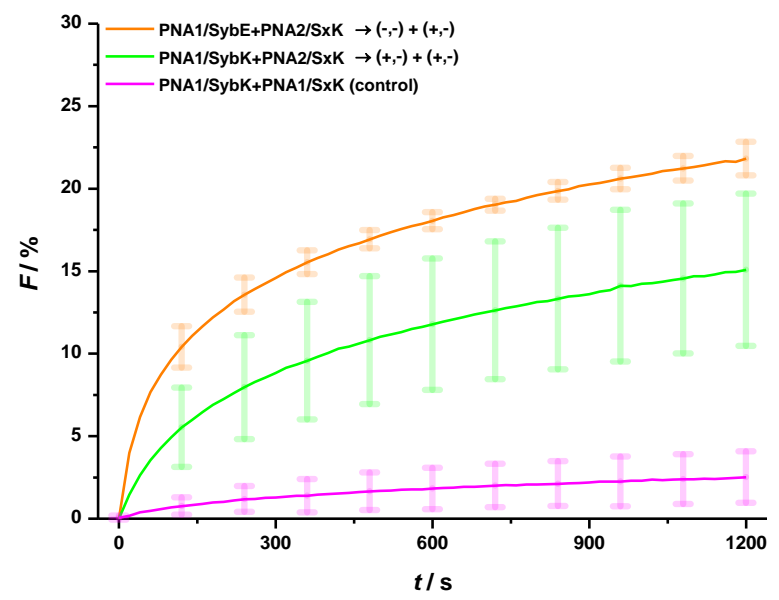

B

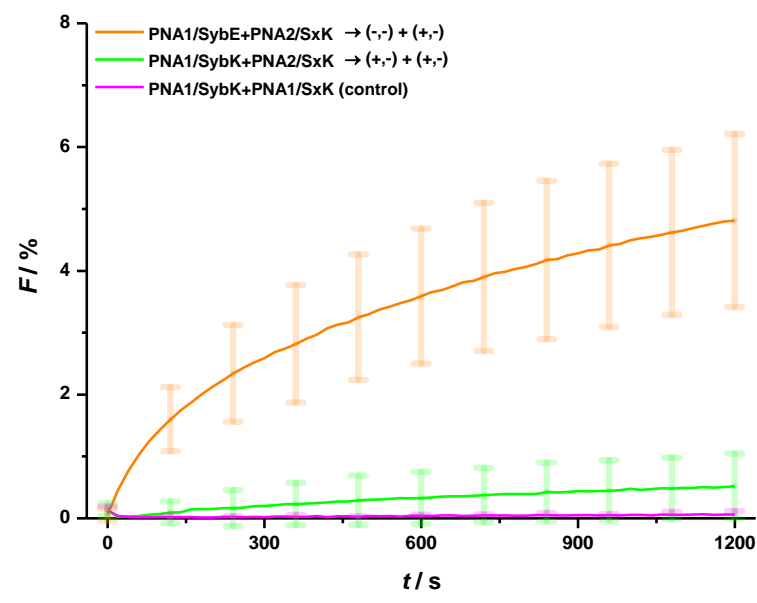

Figure 3.3.1-4 Total and inner leaflet mixing measurements with significant differences in the increase of NBD fluorescence: A) TLM and B) ILM results of antiparallel interacting PNA-LR/TMD peptide hybrids with $C$-terminal lysines and glutamates for the SybTMDs as well as lysines for the SxTMDs (all $C$-terminal aas as carboxylic acids).

\subsection{2) PNA-LR/TMD Peptide Hybrids with $C$-Terminal Am- ides}

For PNA based model peptides with $C$-terminal amides in the parallel recognition mode, significant distinctions are similar compared to $C$-terminal carboxylic acids. The combination PNA1/SybEa + PNA3/SxKa ( 20\%) is again the most fusogenic in this group and is clearly distinguishable from the combinations PNA1/SybKa + PNA3/SxKa $(\sim 8 \%)$ and PNA1/SybEa + PNA3/SxEa ( 5\%) in TLM assays (Figure 3.3.2-1, A). Liposomes carrying SNARE mimetics with $C$-terminal amides are in general less stable than carboxylic acids. Especially the model peptides with lysines as amides destabilised applied membranes (Section 3.4.2). The combination PNA1/SybKa + PNA3/SxKa $(\sim 8 \%)$ exhibit less leaflet mixing compared to its carboxylic acid cognate PNA1/SybK + PNA3/SxK ( 18\%) due to stronger aggregation processes of the labelled proteoliposomes. The negative charge of the side chain of $C$-terminal glutamates as amides seems to counteract destabilising effects of $C$-terminal amides and fusion hampering aggregation is less pronounced. Therefore, the combination PNA1/SybEa + PNA3/SxKa combines stabilising effects owing to $C$-terminal gluta- 
mates, which are advantageous for the detection of leaflet mixing assays through spectrofluorimetry, and destabilising effects of $C$-terminal lysines concerning unlabelled proteoliposomes, which are advantageous for leaflet mixing itself due to membrane perturbing effects. In the case of PNA1/SybEa + PNA3/SxEa destabilising effects of $C$-terminal lysines are missing and fusion efficiency is relative low.

A

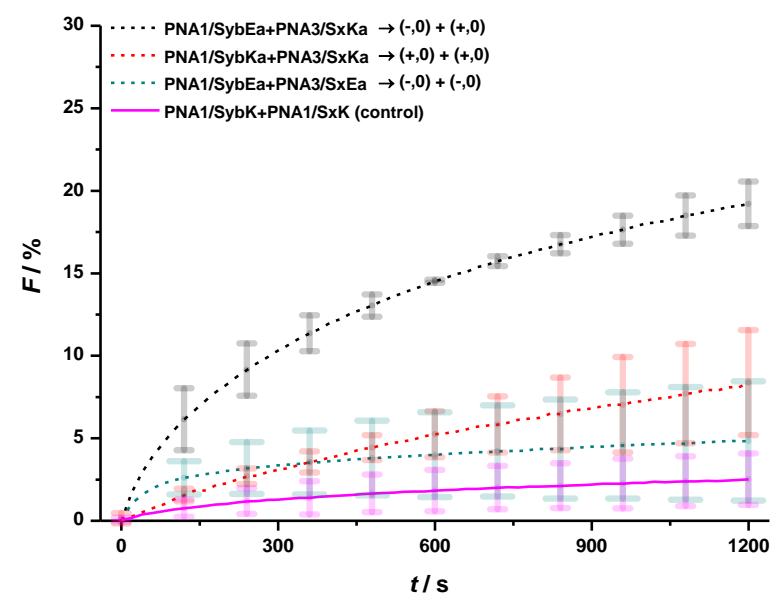

B

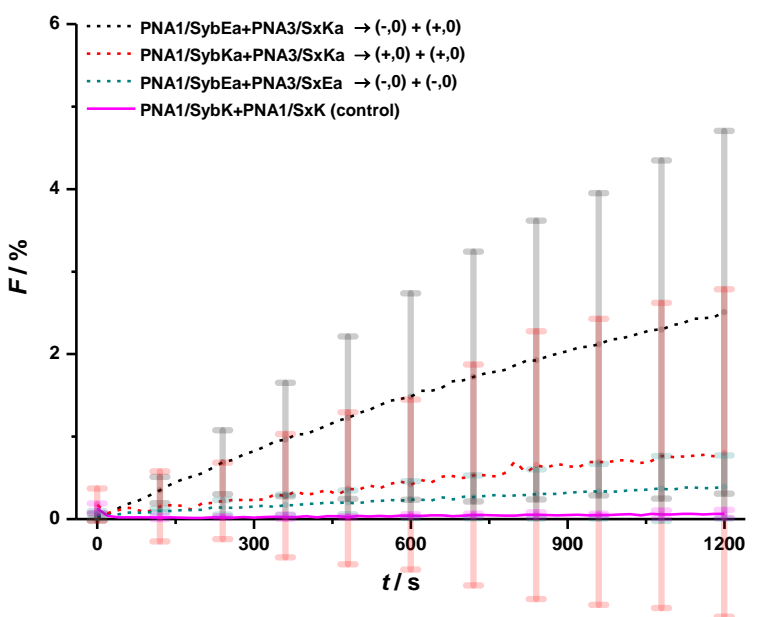

Figure 3.3.2-1 Total and inner leaflet mixing measurements with (significant) differences in the increase of NBD fluorescence: A) TLM and B) ILM results of parallel interacting PNA-LR/TMD peptide hybrids with $C$-terminal glutamates and lysines for the SybTMDs as well as lysines and glutamates for the SxTMDs (all $C$-terminal aas as amides).

Native $C$-terminal aas as amides (PNA1/SybTa + PNA3/SxGa ( 14 \%)) have no $C$-terminal charges. Therefore, the immersion of $C$-terminal ends into the membranes is facilitated. In addition, the lack of charges might lead to flawed insertion of SNARE mimetics destabilising membranes by enhanced surface interactions through the not inserted peptides. This might be another reason for the clear distinction concerning the combination PNA1/SybKa + PNA3/SxKa ( 8 \%, Figure 3.3.2-2, A). However, the main reason is again the high tendency of PNA1/SybKa liposomes to aggregate. This tendency is also given for PNA1/SybTa and PNA3/SxGa liposomes, but the velocity of aggregation was less rapid (Section 3.4.2). Due to the low fluorescence increase in $\operatorname{ILM}(<0.5 \%$, Figure 3.3.2-2, B), the combination PNA1/SybTa + PNA3/SxGa seems to induce only hemifusion, but it is known that this combination can also lead to full fusion. ${ }^{[18]}$ The purity of $15 \%$ of the PNA3/SxGa sample (Appendix, Chromatography Results of PNA-LR/TMD Peptide Hybrids) was relative low compared to other peptides 
of this group (for all peptides $\sim 30 \%$ of purity). Thus, hemifusion might be rather a result of low purity than of structural features of the peptide. In general, variations between the individual ILM measurements of the SNARE mimetics with $C$-terminal amides were especially high and no distinction between the combinations is possible at all (Figures 3.3.2-1, B and 3.3.2-2, B). Again, the low stability of proteoliposomes with SNARE mimetics as amides and accompanied high losses of lipid material during liposome preparation for ILM assays led to weak fluorescence signals during detection. The signal weakness exacerbated the resolution of the ILM measurements.

A

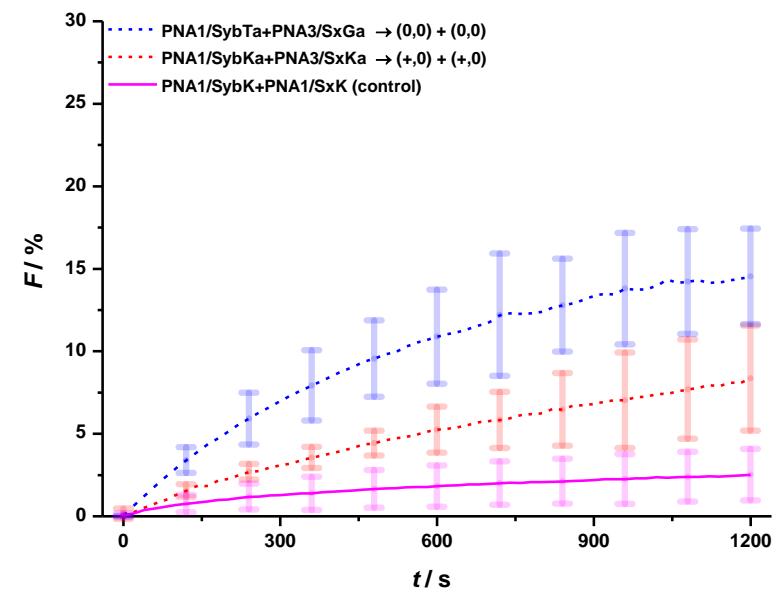

B

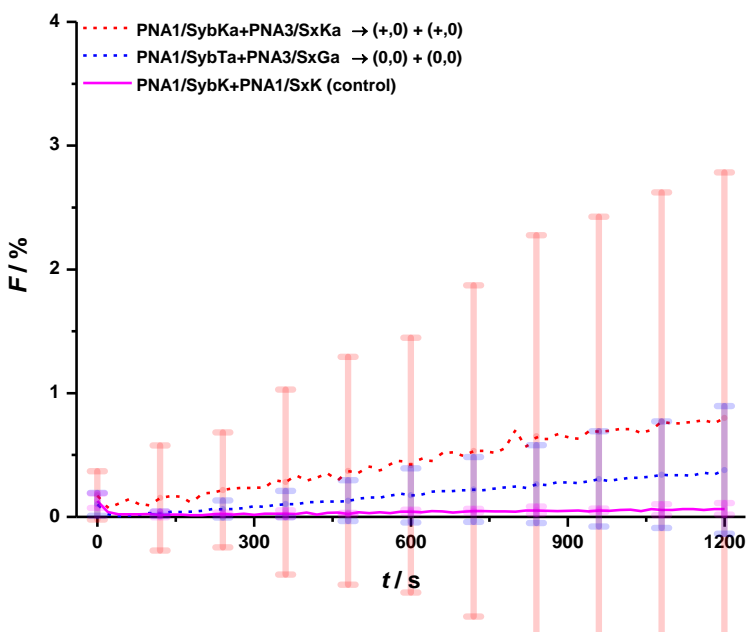

Figure 3.3.2-2 Total and inner leaflet mixing measurements with (significant) differences in the increase of NBD fluorescence: A) TLM and B) ILM results of parallel interacting PNA-LR/TMD peptide hybrids with $C$-terminal threonines and lysines for the SybTMDs as well as glycines and lysines for the SxTMDs (all $C$-terminal aas as amides).

PNA-LR/TMD peptide hybrids as amides in the antiparallel recognition mode were the weakest fusogenes tested in the present study. In TLM assays, all combinations exhibited fluorescence increases smaller than $7 \%$ after 20 min of detection. In ILM measurements, increases were smaller than $1 \%$ (Figure 3.3.2-3, A-B). A significant differentiation was neither in TLM nor in ILM assays possible. It is obvious that these combinations lead only to docking and accompanied unspecific leaflet mixing. Lower degrees of peptide insertion due to missing $C$-terminal negative charges might reduce docking events which is in line with the results of PSM experiments with parallel interacting PNA-LR/TMD peptide hybrids as amides (Section 3.4.6.2). Furthermore, mem- 
brane destabilising effects of not inserted model peptides with $C$-terminal amides (interactions with membrane surfaces) and accompanied liposome aggregation have a negative impact on leaflet mixing itself and its detection through FRET reduction (Sections 3.4.1 and 3.4.2).

A

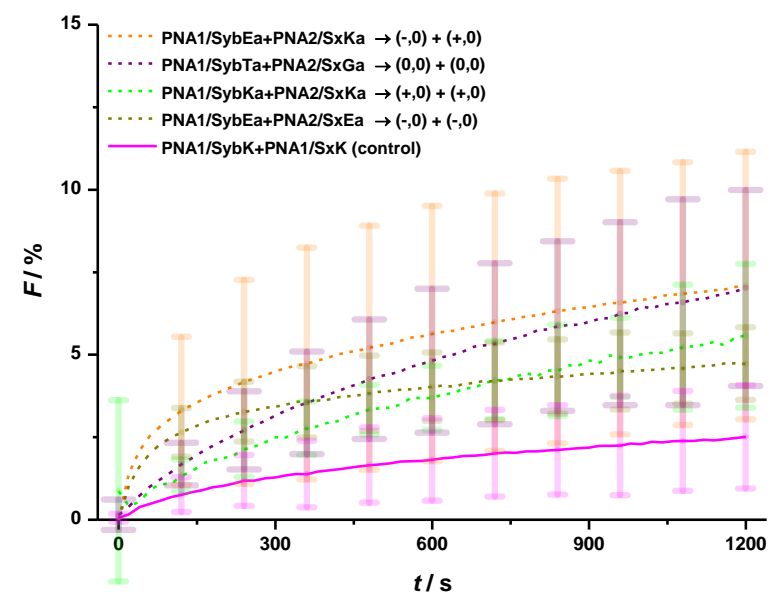

B

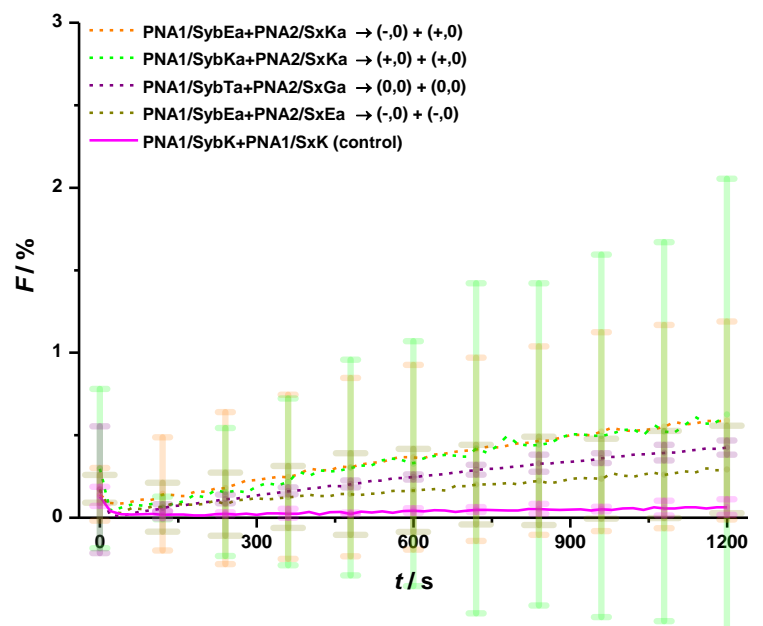

Figure 3.3.2-3 Total and inner leaflet mixing measurements of amide modified PNA-LR/TMD peptide hybrids: A) TLM and B) ILM results of antiparallel interacting model peptides with $C$-terminal amides. For both assays, a significant differentiation between the single combinations is not possible. 


\subsection{3) E3/K3-LR/TMD Peptide Hybrids with $C$-Terminal Car- boxylic Acids}

Like in the case of PNA-LR/TMD peptide hybrids with $C$-terminal carboxylic acids, E3/K3 based model peptides with glutamates and lysines for the SybTMDs and SxTMDs, respectively, induced strongest leaflet mixing ( $40 \%$ fluorescence increase over 20 min detection time). This combination is clearly distinguishable from the combinations E3/SybT + K3/SxG ( 25\%) and E3/SybE + K3/SxE ( 15\%) in TLM and ILM assays (Figure 3.3.3-1, A-B).

A

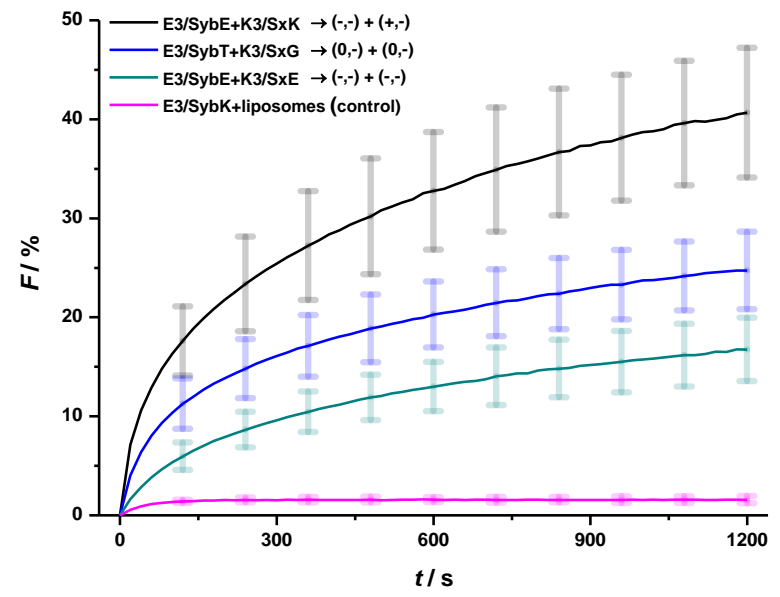

B

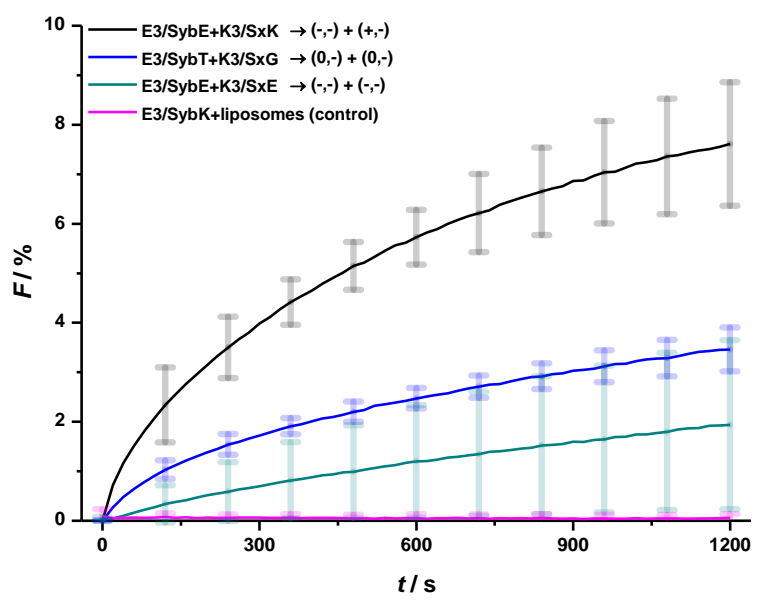

Figure 3.3.3-1 Total and inner leaflet mixing measurements of E3/K3 based SNARE mimetics with (significant) differences in the increase of NBD fluorescence: A) TLM and B) ILM results of parallel interacting E3/K3-LR/TMD peptide hybrids with $C$-terminal glutamates and threonines for the SybTMDs as well as lysines, glycines and glutamates for the SxTMDs (all $C$-terminal aas as carboxylic acids).

The model peptide E3/SybE did not induce aggregation of proteoliposomes and thus it is assumed that the $C$-terminal negative charge of the aa residue is less destabilising than the residual positive charge of lysine (K3/SxK), which induces aggregation (Section 3.4.2). The high stability of labelled E3/SybE liposomes and the membrane perturbating effects of $\mathrm{K} 3 / \mathrm{S} \times \mathrm{KK}$ are probably the main reasons for the high fusogenity of the combination E3/SybE $+\mathrm{K} 3 / \mathrm{SxK}$. The fluorescence increase in ILM assays of this combination was also high ( $\sim \%)$ indicative for full fusion. Native $C$-terminal ends induces full fusion as well, but with a lower tendency ( $\sim 3 \%$ fluorescence increase in ILM assays). $C$-terminal glutamates lead rather to hemifusion, because the emission increase in ILM assays is with $\sim 2 \%$ even lower. Nevertheless, a distinct differentiation 
between the combinations of $\mathrm{E} 3 / \mathrm{SybT}+\mathrm{K} 3 / \mathrm{SxG}$ and $\mathrm{E} 3 / \mathrm{SybE}+\mathrm{K} 3 / \mathrm{S} x \mathrm{E}$ in ILM assays was not possible (Figure 3.3.3-1, B). Whereas the combination E3/SybK + K3/SxK ( $\sim 30 \%$ increase) is clearly distinguishable from the combination E3/SybE + K3/SxE ( $15 \%$ increase) in TLM assays (Figure 3.3.3-2, A). The membrane destabilising effects of $C$-terminal lysines might be one reason for the higher fusogenity of the lysine combination like in the case of the corresponding PNA-LR/TMD peptide hybrids. Another reason might be attractive forces between the $C$-termini which are supposed to support a thinning-widening mechanism of the fusion process (Section 2.7). However, leaflet mixing measurements with PNA based model peptides refute this assumption (Section 3.3.1). The degree of purity of the used peptide samples is probably another reason for the high fusogenity of the lysine combination: E3/SybE and K3/SxE peptides have purities of $\sim 43 \%$ and $\sim 31 \%$, respectively. The lysine modified model peptides have corresponding values of $\sim 18 \%$ and $22 \%$ (Appendix, Chromatography Results of E3/K3-LR/TMD Peptide Hybrids). The higher degree of impurities concerning the lysine combination might induce more points of distortion at the membrane surfaces and therefore supports leaflet mixing processes (Section 3.4.5).

A

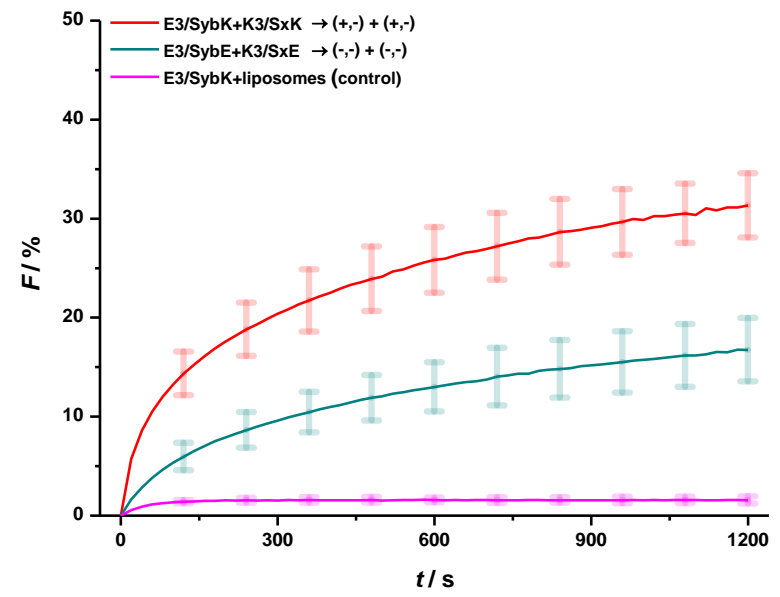

B

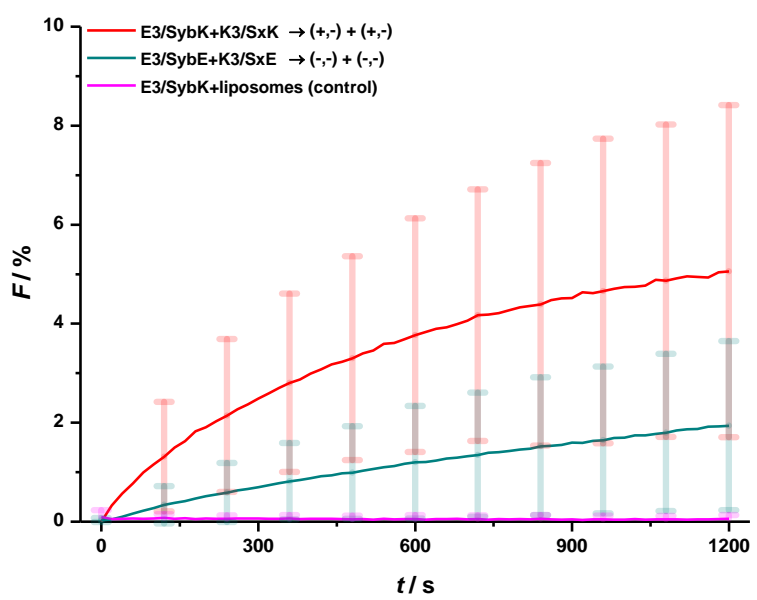

Figure 3.3.3-2 Total and inner leaflet mixing measurements of E3/K3 based SNARE mimetics with (significant) differences in the increase of NBD fluorescence: A) TLM and B) ILM results of parallel interacting E3/K3-LR/TMD peptide hybrids with $C$-terminal lysines and glutamates for the SybTMDs and SxTMDs (all $C$-terminal aas as carboxylic acids).

A clear distinction between the lysine and glutamate combinations with regard to ILM measurements is not possible due to the strong variations between the individual 
measurements (Figure 3.3.3-2, B). However, it can be assumed that the lysine combination has the higher tendency for full fusion ( $\sim \%$ increase in ILM assays) compared to the glutamate combination ( $2 \%$ increase). In conclusion, the E3/K3 recognition system is sufficient to induce full fusion by using native SybT- and SxG-TMDs. Increased negative charges at the $\mathrm{C}$-termini as for glutamate modified model peptides (SybE- and SxE-TMDs) reduce the fusion efficiency because of a higher energy barrier for the immersion of the $C$-termini into the membranes. $C$-terminal lysines (SybK- and SxK-TMDs) seem to rescue and even enhance fusion because of their destabilising effects on membranes.

\subsection{4) E3/K3-LR/TMD Peptide Hybrids with $C$-Terminal Am- ides}

E3/K3 based model peptides with $C$-terminal amides in the parallel recognition mode are the most efficient fusogenes of the present study. Again, the combination of $C$-terminal glutamates with $C$-terminal lysines exhibited the strongest leaflet mixing rates (fluorescence increase of $\sim 45 \%$ ). This combination is clearly distinguishable from the combinations E3/SybTa + K3/SxGa ( 30 \%) and E3/SybEa + K3/SxEa ( 27\%, Figure 3.3.4-1, A). Explanations for the observed fusion behaviour are in line with those of other applied SNARE mimetic groups: Model peptides with $C$-terminal glutamates do not destabilise liposomes due to their residual negative charges. On the other hand, C-terminal lysines destabilise membranes - especially as amides (Section 3.4.2). Stable labelled proteoliposomes are more suitable for the detection of leaflet mixing and destabilised unlabelled proteoliposomes support leaflet mixing processes. The combination E3/SybEa + K3/SxKa combines these features. The reduced fusion efficiency of the combination $\mathrm{E} 3 / \mathrm{SybTa}+\mathrm{K} 3 / \mathrm{SxGa}$ is explainable by the lower stability of the E3/SybTa liposomes compared to the E3/SybEa liposomes. Moreover, glycines of the $\mathrm{K} 3 / \mathrm{S} \times \mathrm{Ga}$ peptides have not the same membrane destabilising effects as lysines of the K3/SxKa peptides. However, native $C$-terminal aas as amides have no charges and this might lead to flawed insertion. Not inserted peptides might additionally destabilise proteoliposomes through interactions with the membrane surface and explain the 
slightly higher leaflet mixing rates of this combination compared to the glutamate combination (not significant). In corresponding ILM assays, the mentioned combinations are not distinguishable from each other due to strong variations between the individual measurements (Figure 3.3.4-1, B).

A

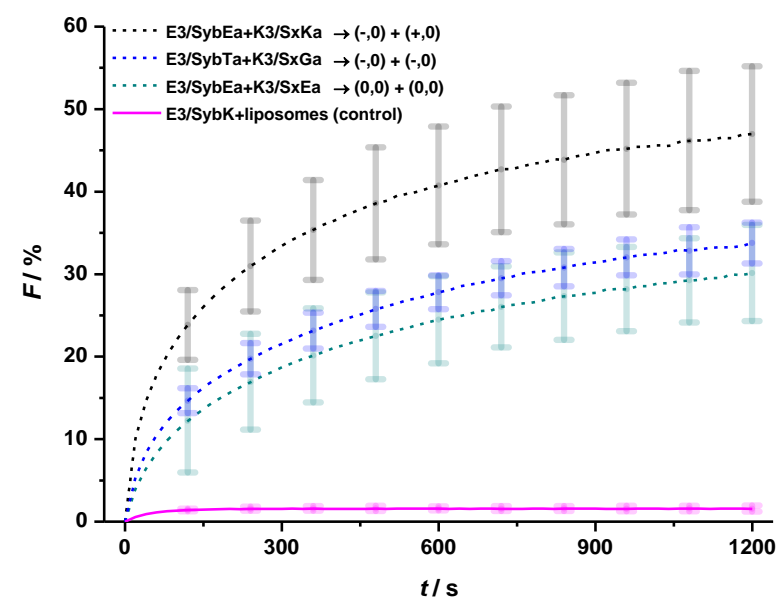

B

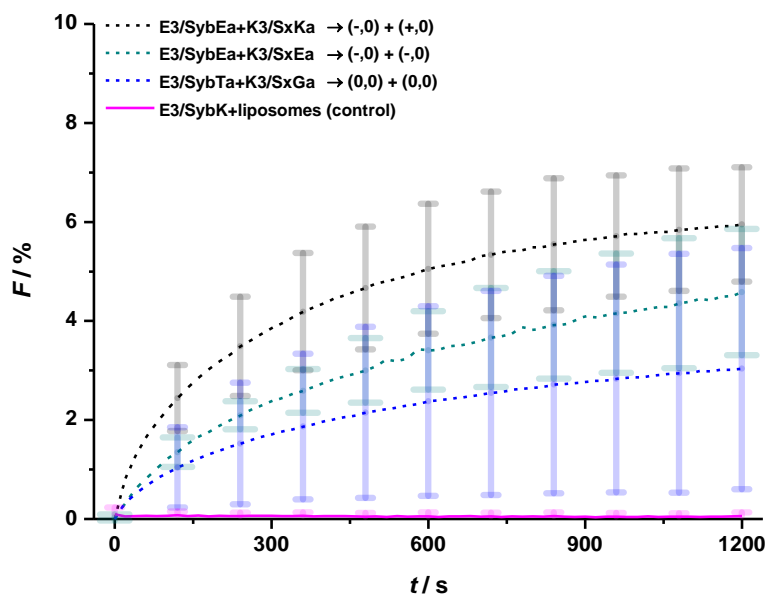

Figure 3.3.4-1 Total and inner leaflet mixing measurements of E3/K3 based SNARE mimetics with (significant) differences in the increase of NBD fluorescence: $\mathbf{A}$ ) TLM and B) ILM results of parallel interacting E3/K3-LR/TMD peptide hybrids with $C$-terminal glutamates and threonines for the SybTMDs as well as lysines, glycines and glutamates for the SxTMDs (all $C$-terminal aas as amides).

In ILM assays for the combination E3/SybEa + K3/SxKa an increase of the fluorescence of $\sim 6 \%$ indicative for full fusion was observed. Other combinations have lower tendencies for full fusion: The fluorescence increases of the combinations $\mathrm{E} 3 / \mathrm{SybTa}+\mathrm{K} 3 / \mathrm{SxKa}$ and E3/SybEa $+\mathrm{K} 3 / \mathrm{SxEa}$ are $~ 3 \%$ and $\sim 4 \%$, respectively. The exchange of the detected fluorescence increases concerning TLM and ILM assays of these combinations (Figure 3.3.4-1, compare A and B) is probably explainable by higher losses of lipid material during the preparation of E3/SybTa liposomes (Section 3.4.3). Moreover, the lysine combination ( $40 \%$ ) can be differentiated from the glutamate combination ( $27 \%$ ) in TLM assays (Figure 3.3.4-2, A). Otherwise, the same combinations are not distinguishable in ILM assays (Figure 3.3.4-2, B). 
A

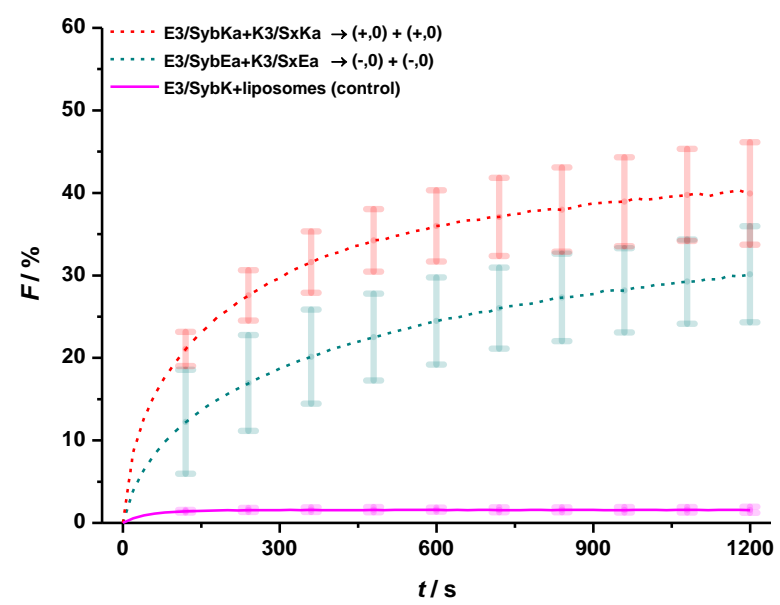

B

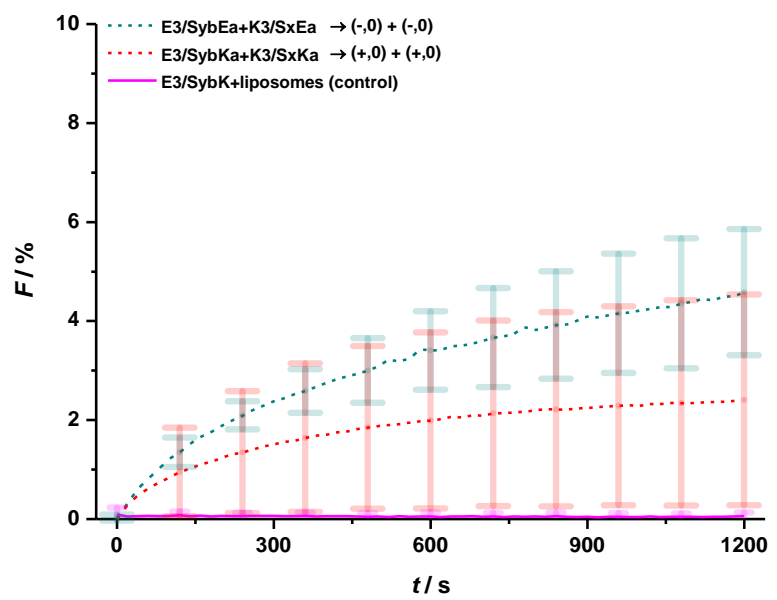

Figure 3.3.4-2 Total and inner leaflet mixing measurements of $E 3 / K 3$ based SNARE mimetics with (significant) differences in the increase of NBD fluorescence: A) TLM and B) ILM results of parallel interacting E3/K3-LR/TMD peptide hybrids with $C$-terminal lysines and glutamates as amides for the SybTMDs and SxTMDs (all $C$-terminal aas as amides).

The destabilising effects on membranes of the lysines are one reason for the higher fusogenity of the combination of peptides with $C$-terminal lysines compared to the glutamate combination. The fact that the tendencies of TLM assays are not reflected in ILM assays is rather explainable by high losses of lipid material during the E3/SybKa liposome preparation for ILM assays (E3/SybKa liposomes aggregate rapidly) than by $C$-terminal structural features of these SNARE mimetics.

\subsection{5) Conclusion of the Results Concerning $C$-Terminal Modifications}

In conclusion, SNARE mimetics with $C$-terminal negative charges yields stable proteoliposomes, but low rates of leaflet mixing. No or positive charges at the $C$-termini of SNARE mimetics result in unstable proteoliposomes, but high rates of leaflet mixing. No charges might result in flawed insertion of the peptides. Partially or not inserted peptides interact with the surfaces of membranes resulting in unstable proteoliposomes. $C$-terminal lysines interact through their residual positive charges and relative long hydrocarbon chains with the phosphate groups and acyl chains of the lipids. These interactions perturb the membrane continuity more than glutamates and native 
aas at the $C$-termini of the model peptides. The instability of proteoliposomes appears as aggregation, which hampers molecular recognition between the membrane bound model peptides and influences the detection of leaflet mixing by spectrofluorimetry. Negatively charged aas might interact with positively charged headgroup residues like choline or ethanolamine, but did not permeate into the membrane in the extent of positively charged $C$-terminal lysines. Thus, aggregation is less prone for SNARE mimetics with $C$-terminal negative charges. ILM assays are especially influenced by unstable labelled proteoliposomes. The aggregation of unstable proteoliposomes leads to a high loss of lipid material during proteoliposome preparation for ILM assays and weak fluorescence signals which reduce the resolution of ILM measurements. Lysines with their positive charges at the amino groups of the side chains and their relatively long residual hydrocarbon chain perturbs membranes as carboxylic acids and as amides, but destabilising effects seem to be stronger when amides are used. In this case, the negative charges of the native carboxylic acid groups are missing and the $C$-terminal ends permeate deeper into the membrane than lysines as carboxylic acids. Furthermore, it is conceivable that $C$-terminal lysines as carboxylic acids (zwitterionic) induce $C$-terminal attractive forces between SybTMDs and SxTMDs, respectively. However, high leaflet mixing rates are also explicable by membrane destabilisation through lysine residues. For instance, PNA-LR/TMD peptide hybrids with $C$-terminal lysines as carboxylic acids, which interact in the antiparallel mode, tend to high leaflet mixing rates as well. Therefore, it is not possible to assign the high fusion rates to attractive forces between the $C$-termini. Beside structural properties of the model peptides other factors like unexpected stoichiometry due to loss of lipid material during extrusion and size exclusion chromatography, different degrees of peptide insertion and different grades of impurities of the peptide samples influence the experimental outcomings.

\section{4) Evaluation of Leaflet Mixing Assays}

In the following sections, problematic issues concerning the applied FRET based leaflet mixing assays are discussed: Crucial points are ILM assays, proteoliposome stability, loss of lipid material during extrusion and size exclusion chromatography, degrees of peptide insertion and the purity of peptide probes. 


\subsection{1) Evaluation of Inner Leaflet Mixing Assays}

For the inner leaflet mixing (ILM) assays, labelled proteoliposomes were treated with sodium dithionite (DT). The reduction of the NBD fluorophores and accompanied loss of fluorescence was determined by means of spectrofluorimetry. The typical decrease of fluorescence was in the range of $60 \%$, whereby it must be noted that $\sim 10 \%$ were induced by dilution due to the addition of DT solution to the proteoliposome suspension (Figure 3.4.1-1, A). After DT treatment, the excess of DT was removed by size exclusion chromatography. However, many of the labelled proteoliposome samples tend to visible aggregation within 30-60 min after extrusion. In the case of PNA-LR/TMD peptide hybrids, proteoliposomes with PNA1/SybK, PNA1/SybKa and PNA1/SybTa peptides were unstable. For E3/K3 based model peptides, proteoliposomes with E3/SybKa and E3/SybTa peptides exhibited high instability (for $100 \%$ of the samples fast visible aggregation was observed). Due to fast aggregation, the loss of lipid material during size exclusion chromatography and therefore the loss of fluorescent probes was especially distinct for these samples. For instance, if one compares the decrease of $\mathrm{Rh}$ fluorescence before and after size exclusion chromatography of stable PNA1/SybT and unstable PNA1/SybKa liposomes, it is obvious that the loss of fluorescence is much higher for the unstable proteoliposomes (Figure 3.4.1-1, B). For stable PNA1/SybT liposomes, the decrease of fluorescence through size exclusion chromatography at $585 \mathrm{~nm}$ was $\sim 60 \%$ and for unstable PNA1/SybKa liposomes $~ 95 \%$.

A

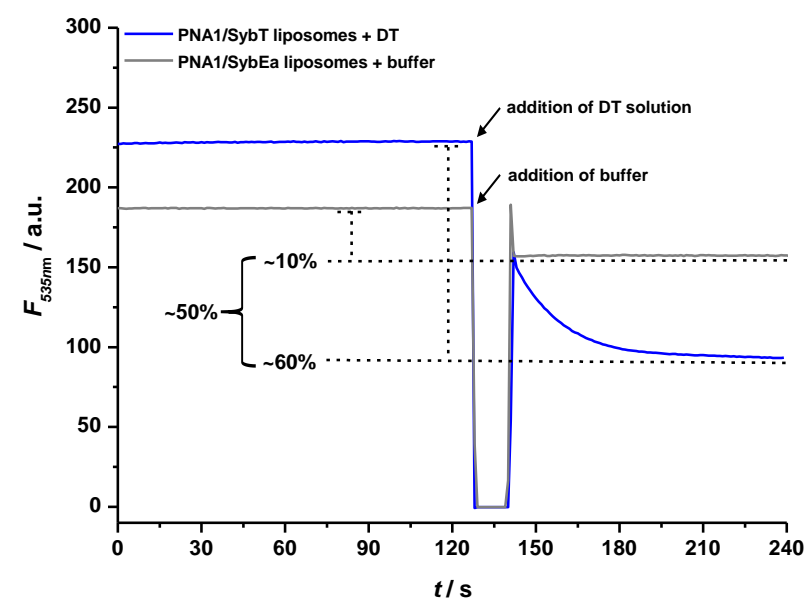

B

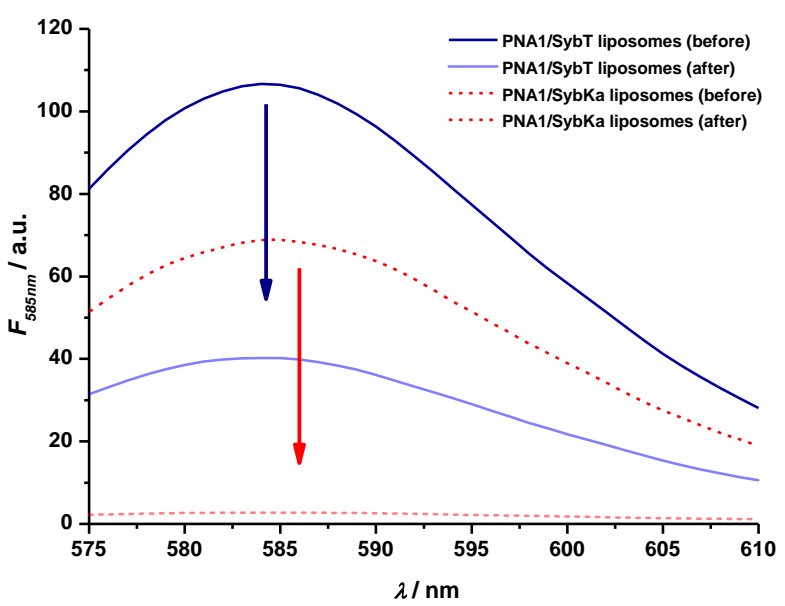

Figure 3.4.1-1 A) Detection of NBD fluorescence during DT treatment. The typical decrease of fluorescence was $\sim 50 \%$. B) Loss of Rh-labelled DOPE during size exclusion chromatography: Fluorescence 
spectra of labelled PNA1/SybT liposomes (stable) before (solid, dark blue) and after (solid, light blue) as well as of labelled PNA1/SybKa liposomes (unstable) before (dashed, dark red) and after (dashed, light red) size exclusion chromatography.

Size exclusion chromatography of other proteoliposome samples led to similar results with regard to aggregation tendencies. Beside the detection of fluorescence of labelled lipids, the loss of lipid material was also evaluated by the determination of the amounts of inorganic phosphate of the phospholipids before and after size exclusion chromatography. ${ }^{[163]}$ Both measurements are in good agreement with each other (Table 3.4.1-1). Further results are presented in the Appendix: Loss of Lipid Material During Proteoliposome Preparation and Size Exclusion Chromatography, Tables A-3 and A-5.

Table 3.4.1-1 Loss of lipids during size exclusion chromatography determined by phosphate tests and Rh-fluorescence.

\begin{tabular}{|l|c|c|}
\hline \multicolumn{1}{|c|}{ sample } & $\begin{array}{c}\text { loss determined by } \text { PO }_{4} \text { test } \\
{[\%]^{\text {] }}}\end{array}$ & $\begin{array}{c}\text { loss determined by Rh-fl. } \\
{[\%]^{\mathbf{b}}}\end{array}$ \\
\hline PNA1/SybE & 55.2 & 55.6 \\
\hline PNA1/SybEa & 89.7 & 64.8 \\
\hline PNA1/SybTa & 86.7 & 81.0 \\
\hline PNA1/SybTa & 73.5 & 73.8 \\
\hline
\end{tabular}

Remarks: a) Loss of lipid material calculated by using determined masses of phosphorus before and after size exclusion chromatography and b) by exploiting the fluorescence intensity at $585 \mathrm{~nm}$ of Rh-DOPE before and after size exclusion chromatography. PNA1/SybE liposomes are tendentially stable, PNA1/SybEa and PNA1/SybTa liposomes are unstable.

The performance of ILM assays for unstable proteoliposomes was problematic, because of the resulting weak fluorescence signals. The detected fluorescence increases of the leaflet mixing curves are in a small range and a differentiation between them is problematic. However, the ILM measurements can be normalised in two different ways: If for normalisation the fluorescence before DT treatment is used, i.e. fluorescence originates from both leaflets, the resolution of the detected emission is low (Figure 3.4.1-2, A) compared to the detection of fluorescence after DT treatment, i.e. fluorescence of NBD originates solely from the inner leaflets. In the latter case, fusion curves can be better distinguished from each other (Figure 3.4.1-2, B). 
A

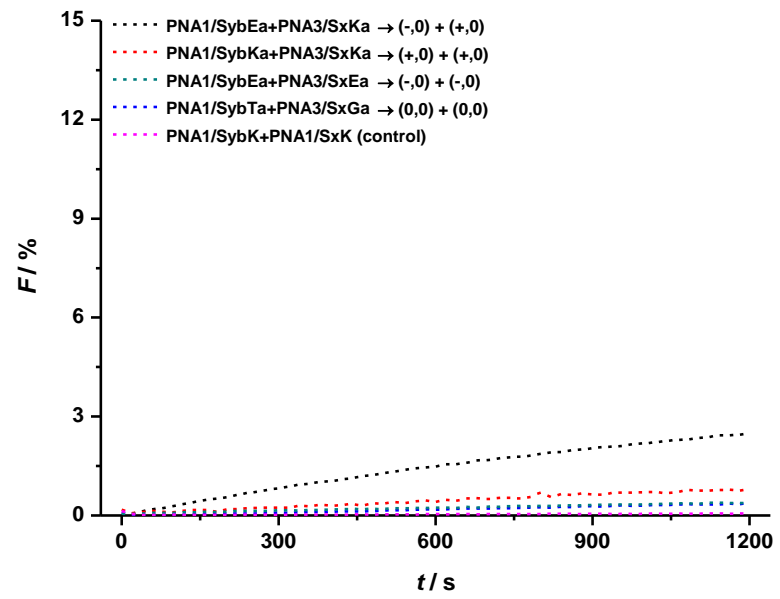

B

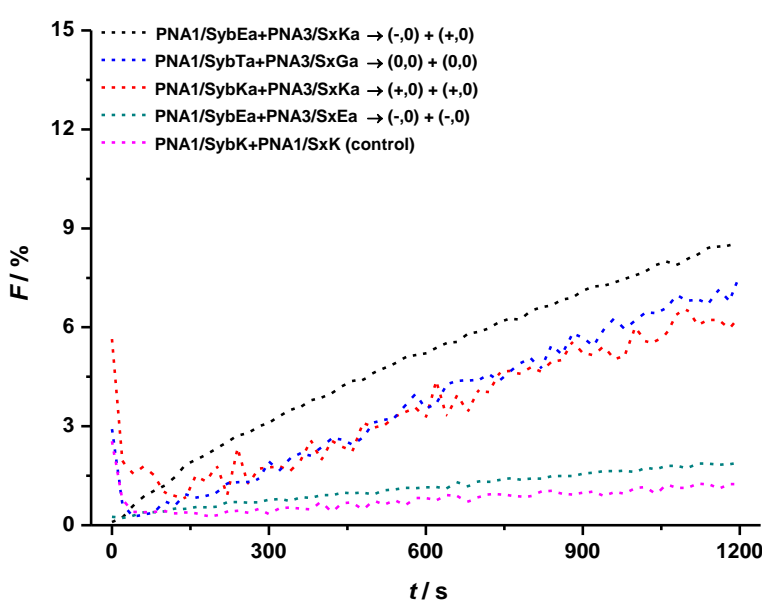

Figure 3.4.1-2 Comparison of two normalisation approaches for ILM assays (same proteoliposome probes were used): Normalisation of the fluorescence increase by using $F_{\max }$ values of labelled proteoliposomes A) before DT induced NBD inactivation of the outer leaflets and B) after NBD inactivation.

However, herein presented results of ILM assays were evaluated by using the first mentioned approach, because of better comparability with TLM assays. Percental values at the $y$-axis are directly related to the corresponding TLM assays. An emission increase of $10 \%$ means that $10 \%$ of both leaflets are mixed. For the second approach, percental values are restricted to inner leaflets, i.e. that an increase of $10 \%$ of emission indicates $10 \%$ of inner leaflet mixing (100\% corresponds to the total abolition of FRET by micellisation of the proteoliposomes through detergent treatment).

\subsection{2) Evaluation of Liposome Stability}

In the previous sections, it has already been mentioned that several proteoliposome samples were unstable, i.e. they tend to aggregate. Turbidity of the proteoliposome suspensions is a visible sign for aggregation. This can be detected by measuring theoptical density of the samples at $550 \mathrm{~nm}$ (not performed). ${ }^{[102]}$ Another possibility to analyse membrane stability is restricted to labelled liposomes. This test can be performed before visible turbidity evolves. NBD/Rh-labelled proteoliposomes were treated with sodium dithionite (DT). This reducing reagent is charged and does not cross the hydrophobic core of membranes, if it is added in appropriate concentrations to the proteoliposome suspensions. ${ }^{[149]}$ The addition induces a drop of fluorescence, because the 
nitro group of NBD-labelled lipids of the outer leaflets is reduced and thereby NBD loses its fluorescent features. Subsequently, the fluorescence reaches a plateau and no further decrease is detected. However, if the proteoliposomes are unstable, a slow and continual decrease of the fluorescence was observed due to membrane defects and/or rupture processes enabling DT to reach NBD-DOPE of the inner leaflets (Figure 3.4.2-1, A-B). It must be noted that flip-flop motions of lipids might be another reason for the decrease of NBD emission after DT treatment, but this process is actually slow. Nevertheless, SNARE-TMDs are known to accelerate lipid translocation and therefore a slight fluorescence decrease after DT treatment might also originates from the inserted model peptides ${ }^{[34,168,169]}$ All labelled proteoliposomes were tested concerning its membrane stability through DT treatment. In Table 3.4.2-1 averaged values (out of three measurements) of fluorescence curves like in Figure 3.4.2-1 are presented. Slopes before DT addition (Sbefore) are closer to zero than afterwards because of the absence of the reducing DT. Additionally, values are rather positive, probably due to slight rupture processes and an accompanied decrease of FRET between NBDand Rh-labelled DOPE.
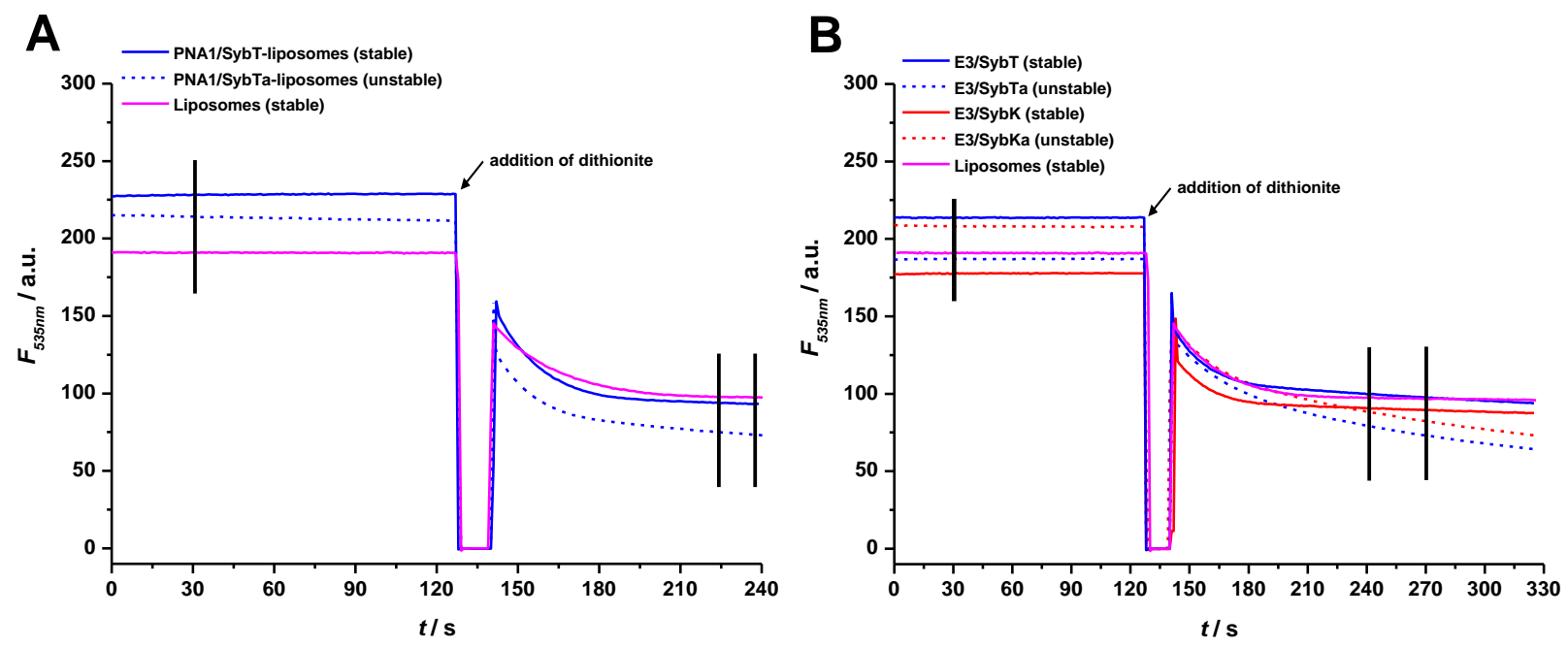

Figure 3.4.2-1 Evaluation of proteoliposome stability through DT treatment: Detection of NBD emission of NBD/Rh-labelled proteoliposomes carrying A) PNA-LR/TMD peptide hybrids and B) E3/K3 based model peptides. Areas limited by black lines were used for slope determination (for calculation see Section 5.7.7.5).

Exceptions from that are liposomes carrying PNA1/SybK and PNA1/SybKa peptides with relative high positive Sbefore (0.097 a.u./s and 0.044 a.u./s, respectively) as well as 
liposomes with $\mathrm{E} 3 / \mathrm{SybE}$ and E3/SybEa with relatively high negative Sbefore (-0.028 a.u./s and -0.026 a.u./s). High positive Sbefore might be explainable by stronger rupture processes due to the destabilising effects of $C$-terminal lysines which interact stronger with the membrane surfaces than the other applied aas. The high negative Sbefore of the E3/SybE and E3/SybEa samples might be explicable by proteoliposomes which adhere to the walls of the cuvette. Proteoliposomes, which stick to glass walls outside the area of detection are not available for further detection. However, this is not a consequence of the peptides E3/SybE and E3/SybE, because PNA1/SybE and PNA1/SybEa (no consequence of the TMDs) as well as other E3 based model peptides (no consequence of the recognition unit E3) did not exhibit this experimental outcomings. Thus, this result is rather a consequence of inherent features of the individual samples than of the model peptide features.

Table 3.4.2-1 Slopes of fluorescence curves before and after DT treatment: Sbefore is the slope within the first $30 \mathrm{~s}$ of the measurement and $S_{\text {after }}$ is the slope within $120-150 \mathrm{~s}$ after the addition of DT.

\begin{tabular}{|l|c|c|}
\hline \multicolumn{1}{|c|}{ liposomes with: } & Sbefore [a.u./s] & Safter [a.u./s] \\
\hline PNA1/SybT & 0.004 & -0.046 \\
PNA1/SybE & 0.005 & -0.024 \\
PNA1/SybK & 0.097 & -0.041 \\
\hline PNA1/SybTa & 0.013 & -0.182 \\
PNA1/SybEa & 0.013 & -0.068 \\
PNA1/SybKa & 0.044 & -0.060 \\
\hline E3/SybT & -0.001 & -0.074 \\
iE3/SybT & 0.005 & -0.031 \\
\hline E3/SybE & -0.028 & -0.034 \\
E3/SybK & 0.008 & -0.054 \\
\hline E3/SybTa & 0.001 & -0.201 \\
\hline E3/SybEa & -0.026 & -0.114 \\
E3/SybKa & -0.013 & -0.172 \\
\hline Pure liposomes & 0.003 & -0.017 \\
\hline
\end{tabular}

Slopes after DT addition ( $S_{\text {after }}$ ) are mainly in line with fast visible aggregation, i.e. labelled liposomes with slopes smaller than -0.040 a.u./s after DT treatment tend to aggregate within 30-60 min after extrusion (compare Table 3.4.2-1 with Table 3.4.2-2). Safter for PNA1/SybEa, E3/SybT, E3/SybK and E3/SybEa are smaller than 
-0.040 a.u./s, i.e. proteoliposomes are partly permeable for DT ions, but exhibit no visible aggregation directly after extrusion. However, long-termed aggregation, i.e. visible aggregation several days or weeks after proeteoliposome preparation, was observed.

Table 3.4.2-2 Visible aggregation of labelled liposomes within 30-60 min after preparation: $n_{\text {total }}$ is the total number of tested liposome probes, $n_{\text {stable }}$ is the number of liposome probes without aggregation, nunstable is the number of liposome probes with aggregation and punstable is the percental amount of unstable liposome probes.

\begin{tabular}{|l|c|c|c|c|}
\hline \multicolumn{1}{|c|}{ liposomes with } & $\boldsymbol{n}_{\text {total }}$ & $\boldsymbol{n}_{\text {stable }}$ & $\boldsymbol{n}_{\text {unstable }}$ & $\boldsymbol{p}_{\text {unstable }}[\%]$ \\
\hline PNA1/SybT & 4 & 3 & $1^{\text {a }}$ & 25 \\
PNA1/SybE & 3 & 3 & 0 & 0 \\
PNA1/SybK & 6 & 0 & 6 & 100 \\
\hline PNA1/SybTa & 9 & 0 & 9 & 100 \\
PNA1/SybEa & 5 & 5 & 0 & 0 \\
PNA1/SybKa & 4 & 0 & 4 & 100 \\
\hline E3/SybT & 4 & 4 & 0 & 0 \\
iE3/SybT & 4 & 4 & 0 & 0 \\
E3/SybE & 7 & 7 & 0 & 0 \\
E3/SybK & 7 & 7 & 0 & 0 \\
\hline E3/SybTa & 6 & 0 & 6 & 100 \\
E3/SybEa & 5 & 5 & 0 & 0 \\
E3/SybKa & 5 & 0 & 5 & 100 \\
\hline pure liposomes & 4 & 4 & 0 & 0 \\
\hline
\end{tabular}

Taking DT measurements and visible aggregation into account, it is possible to subdivide the SNARE mimetics into three classes concerning their destabilising effects on the liposomes:

1) SNARE mimetics with strong destabilising effects exhibit fast aggregation within 30-60min after proteoliposome preparation and negative slopes slighter than -0.040 a.u./s after DT treatment: PNA1/SybK, PNA1/SybKa, PNA1/SybTa, E3/SybTa and E3/SybKa.

2) SNARE mimetics with inter-destabilising effects show no fast aggregation after proteoliposome preparation, but slighter slopes than -0.040 a.u./s after DT treatment: PNA1/SybEa, E3/SybT, E3/SybK and E3/SybEa. 
3) SNARE mimetics with no destabilising effects do not cause aggregation several months after preparation and slopes are higher than -0.040 a.u./s after DT treatment: PNA1/SybE, E3/SybE and iE3/SybT.

It must be noted that this classification is not exclusively attributed to structural features of the SNARE mimetics but also to aspects like different degrees of purity (Section 3.4.5 and the Appendix, Chromatography Results) of the peptide probes and probably incomplete peptide insertion (Section 3.4.4) concerning some proteoliposome probes. It was observed, that the addition of E3/SybT peptides and the LR/TMD of Syb2 to stable liposome suspensions lead to fast visible aggregation indicative for the destabilising effects of the LR/TMD of Syb2 probably due to interactions with the membrane surface. The same effects are conceivable for peptides which are not correctly reconstituted during proteoliposome preparation.

For unlabelled proteoliposomes with SxLR/TMD based model peptides, the stability was solely evaluated by means of fast visible aggregation (Table 3.4.2-3). 
Table 3.4.2-3 Visible aggregation of unlabelled liposomes within 30-60 min after preparation: $n_{\text {total }}$ is the total number of the tested liposome probes, $n_{\text {stable }}$ is the number of liposome probes without aggregation,

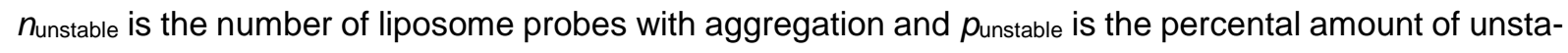
ble liposome probes.

\begin{tabular}{|l|c|c|c|c|}
\hline \multicolumn{1}{|c|}{ liposomes with } & $\boldsymbol{n}_{\text {total }}$ & $\boldsymbol{n}_{\text {stable }}$ & $\boldsymbol{n}_{\text {unstable }}$ & punstable [\%] \\
\hline PNA2/SxG & 4 & 4 & 0 & 0 \\
\hline PNA2/SxE & 3 & 3 & 0 & 0 \\
\hline PNA2/SxK & 8 & 0 & 8 & 100 \\
\hline PNA2/SxGa & 3 & 0 & 3 & 100 \\
\hline PNA2/SxEa & 3 & 3 & 0 & 0 \\
\hline PNA2/SxKa & 9 & 2 & 7 & 78 \\
\hline PNA3/SxG & 4 & 3 & $1^{a}$ & 25 \\
\hline PNA3/SxE & 3 & 3 & 0 & 0 \\
\hline PNA3/SxK & 9 & 0 & 9 & 100 \\
\hline PNA3/SxGa & 9 & 0 & 9 & 100 \\
PNA3/SxEa & 3 & 3 & 0 & 0 \\
\hline PNA3/SxKa & 8 & 0 & 8 & 100 \\
\hline PNA1/SxK & 4 & 0 & 4 & 100 \\
\hline K3/SxG & 5 & 0 & 5 & 100 \\
\hline K3/SxE & 6 & 2 & 4 & 67 \\
\hline K3/SxK & 4 & 0 & 4 & 100 \\
\hline K3/SxGa & 6 & 0 & 6 & 100 \\
\hline K3/SxEa & 4 & 0 & 4 & 100 \\
\hline K3/SxKa & 9 & 0 & 9 & 100 \\
\hline
\end{tabular}

PNA-LR/TMD peptide hybrids with $C$-terminal carboxylic acids are stable except for peptides with $C$-terminal lysines (PNA1/SxK, PNA2/SxK and PNA3/SxK). PNA based SNARE mimetics with $C$-terminal amides are rather unstable. However, peptides with $C$-terminal glutamates as amides (PNA2/SxEa and PNA3/SxEa) are stable. PNA sequences have obviously no influence on aggregation processes, because SNARE mimetics with the same LRs/TMDs but different PNA sequences affect liposomes in the same extent. Concerning $C$-terminal modifications, E3/K3-LR/TMD peptide hybrids have the same effects as PNA based model peptides, i.e. $C$-terminal lysines and/or amides are destabilising and glutamates and/or carboxylic acids are not destabilising. Intriguingly, all K3-peptide hybrids are unstable independent on their $C$-termini. In conclusion, instabilities of all applied proteoliposomes are justifiable by following aspects: 
1) $C$-terminal lysines and lysines of the $K 3$ recognition units interact with the hydrophilic headgroups and hydrophobic tails of the phospholipids (snorkelling interactions). These interactions might shape and thereby perturb membrane continuity. ${ }^{[166,167]}$

2) $C$-terminal amides delete the $C$-terminal negative charges of carboxylic acid groups. In native LR/TMD sequences of Syb2 and Sx1A (SybT and SxG) these charges might help to position TMDs within membranes, because they prefer the hydrophilic environment at the surface of membranes and the negative charges are obviously not tolerated in the hydrophobic core of the membranes. Thus, carboxylic acid groups might act as a $C$-terminal TMD positioner together with the charged aa of the LRs as $N$-terminal TMD positioner ( $C$-terminal aa of the LRs of Syb2 and Sx1A is lysine). The lack of $C$-terminal charges might result in flawed insertion of the TMDs, i.e. the $C$-termini might be rather located within the hydrophobic core than at the surface of the membranes or peptides are in no sense inserted. Thereby, TMDs perturb membranes through stronger surface interactions resulting in a membrane collapse. Aggregation processes seem to be enhanced by $C$-terminal lysines which interact strongly with phospholipids. $C$-terminal glutamate as amide offers a negative charge through its side chain, which replaces the function of the negative charge of the native carboxylic acid group as a $C$-terminal TMD positioner. In this way, SNARE mimetics with $C$-terminal glutamate destabilise membranes less than lysine, threonine or glycine as $C$-terminal amides.

3) For all fusion assays, not purified peptides were used. The concentration determination of peptide stock solutions used for proteoliposome preparation were performed by UV-spectroscopy. However, calculated concentrations using the LAMBERT-BEER law might be misleading, because impurities absorb UV light at chosen wavelengths as well as completely synthesised peptides. Thus, two probes with identical determined concentrations might include different amounts of entirely synthesised peptides and amounts of impurities, which are added during the proteoliposome preparation. For the applied model peptides, impurities probably consist mainly of LR/TMDs of Syb2 and Sx1A with parts of the recognition units, because the possibility for deleted sequences during SPPS increases with increasing peptide chain lengths (synthesis starts at $C$-terminal 
TMDs). In the case of PNA-LR/TMD peptide hybrids, nucleobases and tryptophan contribute mainly to the detected absorption at $260 \mathrm{~nm}$. For instance, if one limits the absorption of the PNA1 sequence to thymine nucleobases and ignore other nucleobases, three TMDs with one thymine instead of one completely synthesised PNA1-SybLR/TMD peptide will be added during the preparation of proteoliposomes, because the PNA1 sequence includes three thymines (Figure 2.6.2.1-1). In the case of E3/K3 based model peptides, circumstances are slightly different, because the concentrations of these peptides are determined by using the absorption of tryptophan, tyrosine and cysteine at $280 \mathrm{~nm}$. Therefore, the absorption used for concentration determinations has its origin exclusively in the LRs/TMDs with regard to (i)E3-SybLR/TMD peptides. For these peptides, indeed rather supposed amounts of TMDs are added during liposome preparation, but the TMDs carry probably no or incomplete recognition motifs. For K3-SxLR/TMD peptides, the situation is comparable to those of PNA based model peptides, because the absorption originates mainly from $\mathrm{N}$-terminal tryptophans (Figure 2.6.2.2-1). In conclusion, for crude PNA-LR/TMD peptide hybrids probably more LRs/TMDs are added than supposed. These peptide fragments might additionally destabilise membranes. In the case of (i)E3-SybLR/TMD peptides, the supposed amounts of LRs/TMDs are probably added, but with less recognition units as supposed. However, K3-SxLR/TMD peptides are rather added with amounts of impurities like for PNA based model peptides. Therefore, liposomes with (i)E3-SybLR/TMD peptides are tendentially more stable than PNA and K3 based model peptides.

Pure liposomes are the most stable probes, because of the lack of membrane destabilising peptides. There were no hints for visible aggregation processes even after months (for DLS measurements see Figure 3.4.6.1-2, A). 


\subsection{3) Loss of Lipid Material During Proteoliposome Prep- aration}

The supposed ratio of 1 to 4 of labelled to unlabelled liposomes is optimal for lipid mixing assays, i.e. applying this ratio lead to the highest increase of donor emission (NBD) during the leaflet mixing assays with the same peptide combination. ${ }^{[170]}$ However, it is not sufficient to limit the determination of the liposome ratio to the used volumes of liposome suspensions for the performance of leaflet mixing assays, because during the preparation of proteoliposomes different amounts of lipid material are restrained by the extrusion apparatus. Rather, it is necessary to determine the mass of phosphorus of the suspensions. This value is proportional to the amounts of phospholipids and to the number of size-homogeneous proteoliposomes. The determination of phosphorus amounts of $\mathrm{iE} 3 / \mathrm{SybT}$ and $\mathrm{K} 3 / \mathrm{S} \times \mathrm{G}$ liposome suspensions revealed that $10 \mu \mathrm{L}$ of the $\mathrm{iE} 3 / \mathrm{SybT}$ liposome suspension contain $0.99 \mu \mathrm{g}$ of phosphorus. The $\mathrm{K} 3 / \mathrm{S} \times \mathrm{G}$ suspension contained less phosphorus $(0.77 \mu \mathrm{g}$ phosphorus $/ 10 \mu \mathrm{L})$. Thus, $10 \mu \mathrm{L}$ of the labelled $\mathrm{iE} / \mathrm{SybT}$ liposome suspension combined with $160 \mu \mathrm{L}$ of the unlabelled K3/SxG liposome suspension (K3/SxG liposome suspension was diluted in the ratio 1 to 3 due to better storage conditions concerning proteoliposome stability) did not reflect the supposed liposome ratio of 1 to 4 (Appendix, Loss of Lipid Material During Proteoliposome Preparation and Size Exclusion Chromatography, Table A-5).

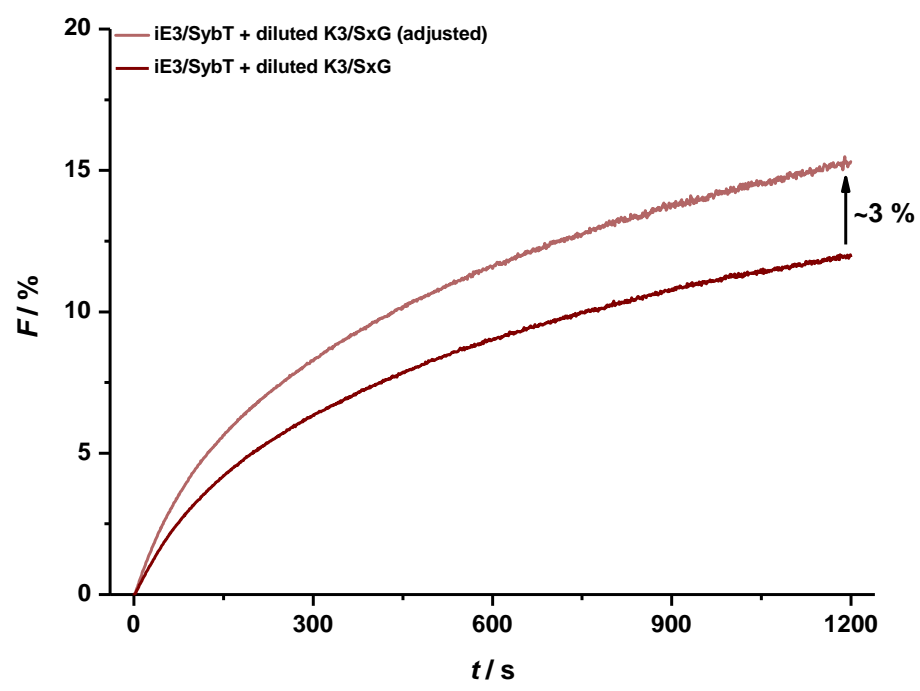

Figure 3.4.3-1 Total leaflet mixing of liposomes decorated with iE3/SybT and K3/SxG peptides: Dark brown curve represents the 1:4 ratio limited to the added volumes of labelled and unlabelled proteoliposomes. The light brown curve represents the adjusted lipid ratio. To reduce lipid aggregation processes 
and gain time for phosphate tests, the $\mathrm{K} 3 / \mathrm{S} x \mathrm{G}$ liposome probe was diluted with fusion buffer $(1: 3)$. Measurements were performed ca. $24 \mathrm{~h}$ after extrusion of $\mathrm{iE} 3 / \mathrm{SybT}$ and K3/SxG liposomes.

In this experiment, the actual ratio was 1 to 3.1 . By increasing the added volume of the K3/SxG liposome suspension from $160 \mu \mathrm{L}$ to $205 \mu \mathrm{L}$ the supposed ratio of 1 to 4 was achieved. For the adjusted liposome ratio, the increase of NBD emission was $3 \%$ higher than for the not adjusted ratio (Figure 3.4.3-1). These results underline that it is necessary to determine the mass of phosphorus for each proteoliposome suspension to ensure that the actual liposome ratio match the supposed ratio.

Like mentioned before, for ILM assays it is necessary to delete the amount of NBD fluorescence originating from the outer leaflets of the membrane by sodium dithionite (DT). The excess of DT must be removed before starting the ILM assays. By size exclusion chromatography, DT is separated from the proteoliposomes. However, also small proteoliposomes and micelles, which are formed during the proteoliposome preparation or subsequently formed voluminous lipid aggregates are restrained. The result is a reduced lipid concentration, which influences the supposed lipid ratio for ILM assays of 1 to 4 . Therefore, it is necessary to adjust the lipid ratio by using the results of phosphate determination assays. Additionally, it is possible to determine the loss of lipids via the loss of the fluorescent probe Rh-DOPE by spectrofluorimetry (Figure 3.4.1-1, B and Table 3.4.1-1).

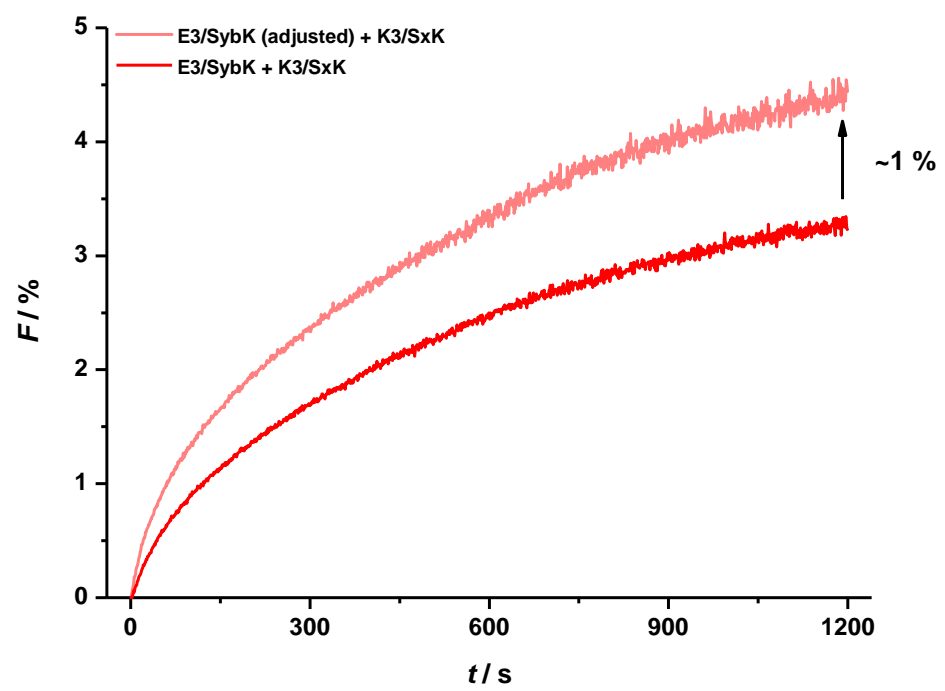

Figure 3.4.3-2 ILM of liposomes decorated with E3/SybK and K3/SxK peptides: Dark red curve represents the 1:4 ratio limited to the added volumes of labelled and unlabelled proteoliposomes. Light red curve represents the adjusted lipid ratio considering solely the E3/SybK liposome sample. Both measurements were performed $1 \mathrm{~d}$ after the preparation of proteoliposomes. 
In Figure 3.4.3-2 the increases of NBD fluorescence indicative for inner leaflet mixing are presented: The merging proteoliposomes were decorated with E3/SybK (labelled proteoliposomes) and K3/SxK (unlabelled proteoliposomes) peptides. In one case, the supposed ratio was solely adjusted by the added volumes of proteoliposome suspensions. In the other case, the added volume of the E3/SybK liposome suspension was adjusted by means of phosphorus determination. Through the adjustment, a higher increase of NBD emission was observed, though solely the added amounts of the E3/SybK liposome suspension was considered. It is expected that the additional consideration of unlabelled K3/SxK liposomes would lead to even stronger leaflet mixing.

\subsection{4) Evaluation of the Insertion Degree of SNARE Mimet- ics in Model Membranes}

It is doubtful that all used SNARE mimetics are inserted to the same levels in the model membranes and are arranged correctly as integral peptides. For instance, SNARE mimetics without $C$-terminal charges (SybTa- and SxGa-TMDs) might insert partially, i.e. span the membrane only partially, or they inserted in no sense. Therefore, it is crucial to examine the degree of peptide insertion. Density-gradient centrifugation and fluorescent probes were used to search into this crucial point of experiments concerning membrane fusion. 


\subsubsection{1) Evaluation by Density-Gradient Centrifugation}

Density-gradient centrifugation and subsequent SDS-PAGE was applied to examine whether the SNARE mimetics are inserted in the liposome membranes. Proteoliposomes were ultra-centrifuged in a medium of layered units of different density. During the centrifugation, liposomes accumulate in layers of low density. Whereas not inserted peptides accumulate in layers of high density (Figure 3.4.4.1-1).

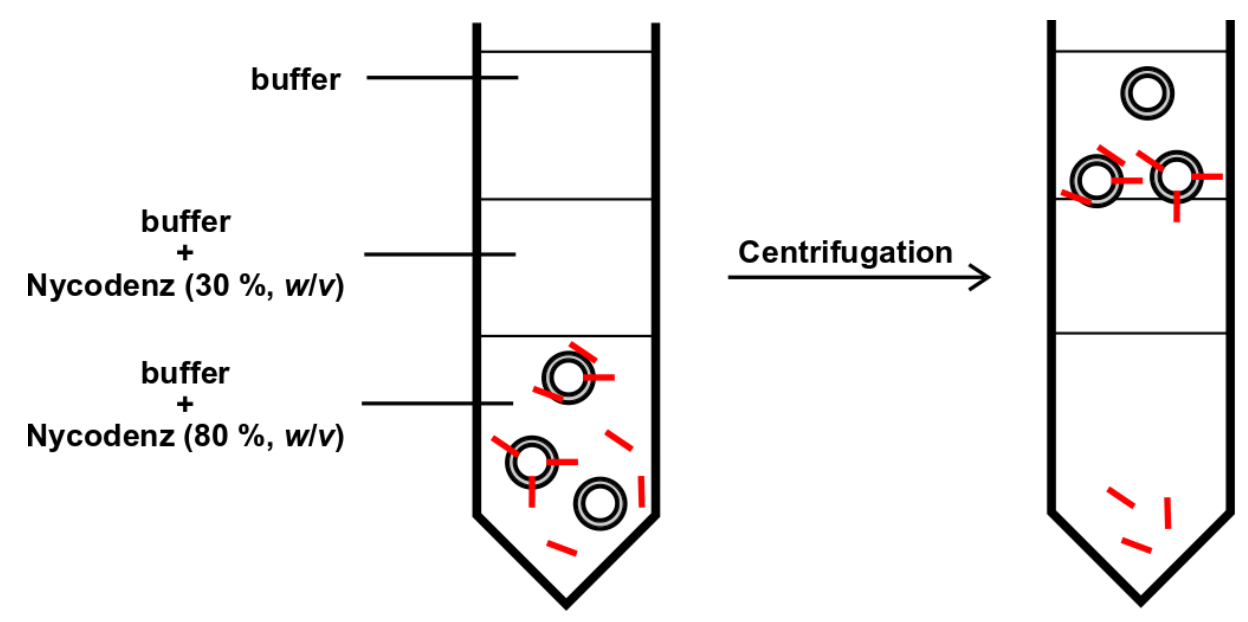

Figure 3.4.4.1-1 Illustration of density-gradient centrifugation: Nycodenz is used to establish different layers of density when dissolved in buffer. Black circles and red bars illustrate liposomes and model peptides, respectively.

The content of the test tubes was fractionated after centrifugation and SDS-PAGE was performed. It is assumed that the detection of peptides via silver-staining in fractions of low density (pure buffer) is an evidence for the insertion of peptides in the liposome membranes. However, it is also possible that peptides adsorb at the surface of the liposome membranes or that they are only partially inserted. Beside the examination of proteoliposomes prepared through standard procedures (Section 5.5.1), proteoliposomes with the threefold amounts of peptide $(n=37.5 \mathrm{nmol})$ were prepared to provoke failed insertion. Furthermore, peptides dissolved in TFE were subsequently added to pure liposomes to test if peptides are adsorbed at the surface of the liposomes. It is known that low concentrations of TFE in liposome suspensions $(<30 \%, v / v)$ have no effect on the bilayer structure. ${ }^{[171]}$ For standardly prepared proteoliposomes, the presence of peptides was indeed proved only for fractions of low density indicative for peptide insertion (Figure 3.4.4.1-2, A). However, liposome samples with an excess of peptides and subsequently added peptides exhibit similar patterns, i.e. peptides were also 
detected in fractions of low density (Figure 3.4.4.1-2, B-C). Therefore, this experiment was not suitable to examine correct peptide insertion.

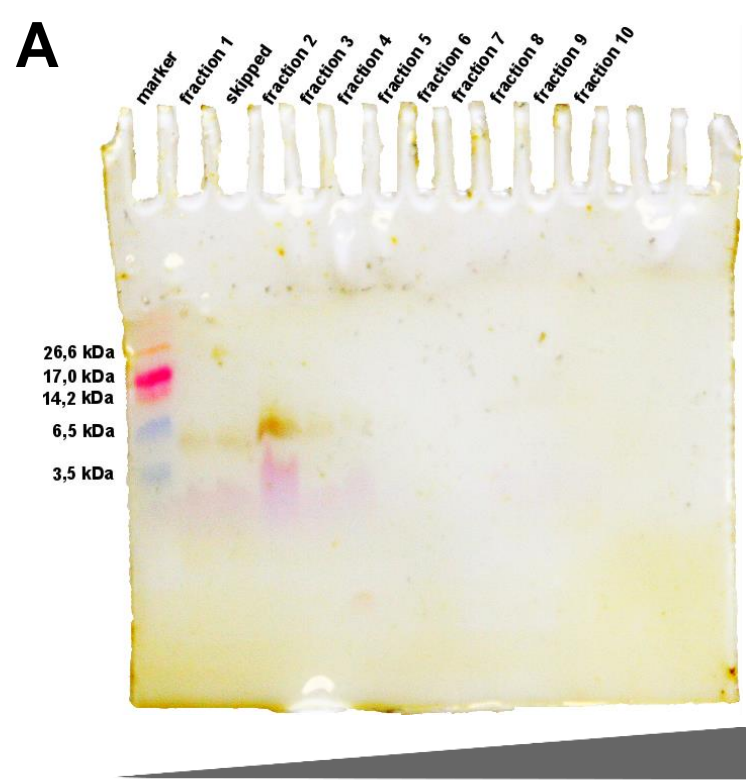

density gradient

B

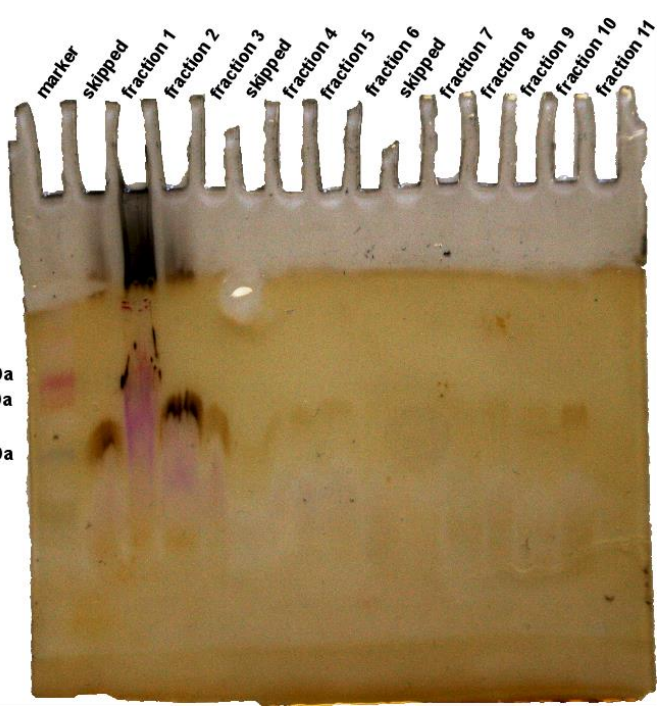

density gradient
C

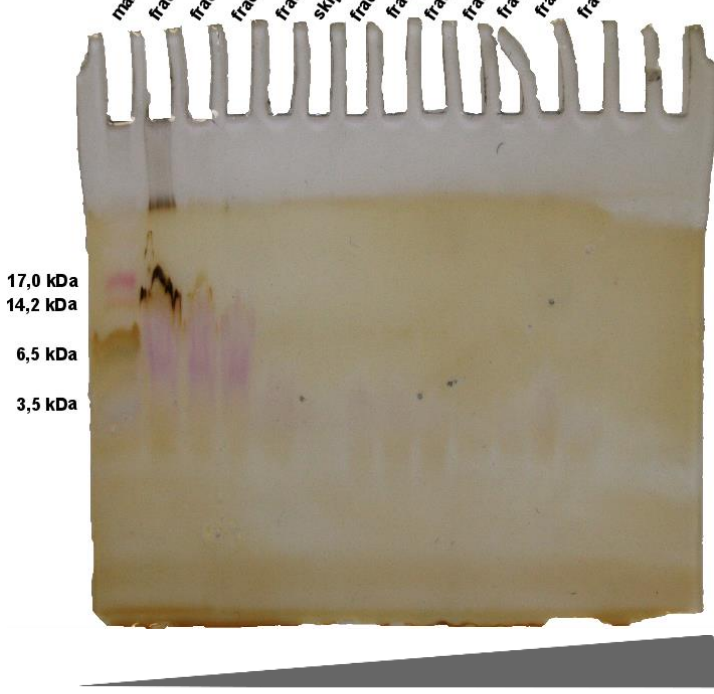

density gradient

Figure 3.4.4.1-2 Images of SDS-PA gels: A) Standardly prepared E3/SybT liposomes. B) Standardly prepared E3/SybT liposomes with the threefold amounts of E3/SybT peptides. C) Pure liposomes with E3/SybT peptides added after liposome preparation. Visualisation of peptides was achieved via silver-staining. Smearing of the peptide bands in B) and C) occurred probably due to overloading and high lipid contents (formation of peptide/lipid aggregates). 
One possible method to overcome the problem concerning peptides adsorbed the surface of membranes is the application of sodium carbonate. By treatment with carbonate, liposomes are converted into membrane sheets. During this process, peripherally associated peptides are released, whereby integrally associated peptides remain within the membrane sheets. Through centrifugation the peripheral peptides are separated from integral peptides. ${ }^{[172]}$

\subsubsection{2) Evaluation by Fluorescent Probes}

Another approach for the determination of peptide insertion is the usage of fluorescently labelled recognition motifs which bind to membrane bound peptides. After the removal of unbound fluorescent probes via dialysis, it might be possible to determine a relative exact amount of SNARE mimetics which are available for molecular recognition. In a first attempt, the K3 recognition motif was labelled with NBD (NBD-K3) at its $N$-terminal end. Furthermore, E3/SybT liposomes were prepared. In buffer dissolved NBD-K3 was added $\left(n_{\text {NBD-K3 }}=2.5 \mathrm{nmol}\right.$ ) to standardly prepared E3/SybT liposomes $\left(n_{\text {Eз/Syb }}=2.5 \mathrm{nmol}\right)$. It was assumed that $60 \%$ of the membrane bound peptides are directed towards the exterior of the proteoliposomes and thus are available for molecular recognition $\left(n_{\mathrm{av} E 3 / \mathrm{Syb}}=1.5 \mathrm{nmol}\right) .{ }^{[170]}$ After sufficient time for the binding of NBD-K3 to the E3 recognition units, it was attempted to remove the excess of unbound NBD-K3 by dialysis (Figure 3.4.4.2-1).
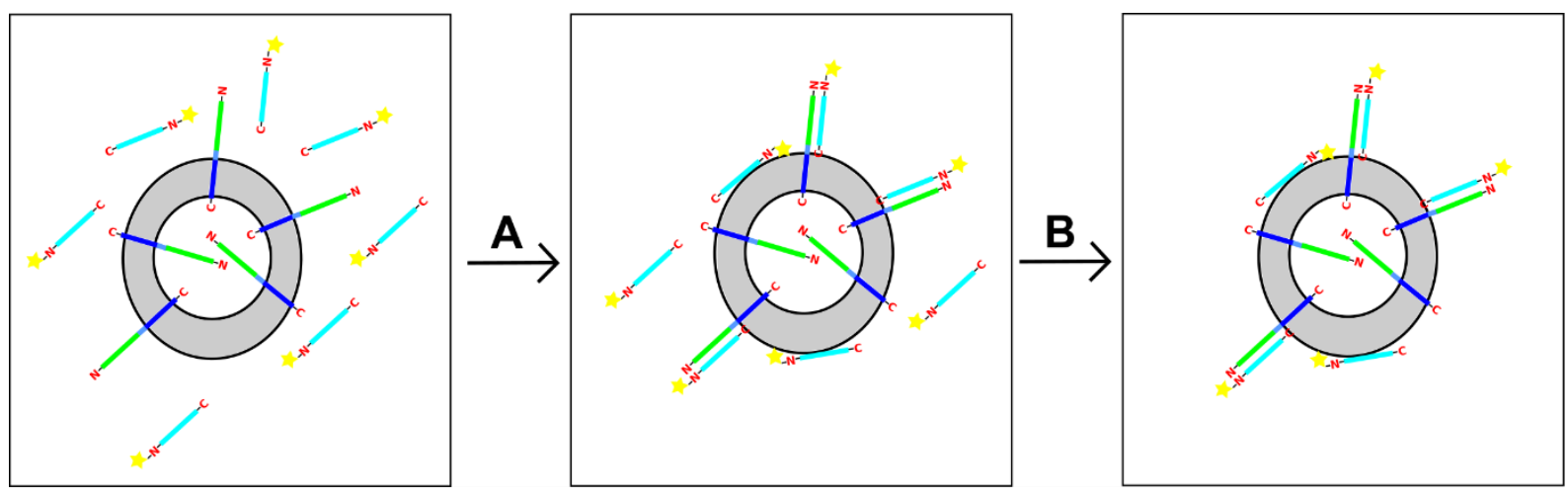

Figure 3.4.4.2-1 Illustration of the peptide insertion test based on fluorescently labelled K3 recognition motifs: A) Molecular recognition between NBD-K3 and available E3/SybT peptides as well as the adsorption of NBD-K3 at the membrane surface. B) Removal of unbound NBD-K3 peptides through dialysis. Colour code: SybT-LR/TMD (blue), E3 recognition unit (light green), K3 recognition unit (turquoise), NBD (yellow star), $N$-and $C$-termini (red letters $\mathrm{N}$ and $\mathrm{C}$ ). 
It must be noted that during liposome preparation (extrusion) and dialysis $47 \%$ and $27 \%$ of the lipid material were lost, respectively (determined by phosphate tests). It was assumed, that the loss of lipid material is proportional to the loss of E3/SybT peptides. Thus, theoretically only $0.58 \mathrm{nmol}$ of the E3/SybT peptides were available after dialysis. For fluorescence measurements, $9.1 \%$ of the dialysis formulation was used (nused $=0,053 \mathrm{nmol}$ ). By the establishment of calibration curves, it was attempted to determine the amount of NBD-K3 which binds to the available E3/SybT peptides of the proteoliposomes. It was calculated, that $0.008 \mathrm{nmol}$ NBD-K3 bound to E3/SybT peptides, i.e. $15.1 \%$ of the membrane bound peptides directed to the exterior were available for molecular recognition. However, through control experiments it was also ascertained that the dialysis procedure removed only $\sim 50 \%$ of the NBD-K3 peptides (dialysis without liposomes). Furthermore, it is known that K3 peptides interact strongly with membranes, i.e. membranes compete with E3 recognition units for binding of NBD-K3 (Figure 3.4.4.2-1). ${ }^{[167]}$ Another problematic issue is the increase of NBD fluorescence due to elevated hydrophobicity of the environment. ${ }^{[173]}$ Therefore, the amount of membrane bound NBD-K3 exhibit probably stronger fluorescence than E3/SybT bound fluorescent peptides and its contribution to total fluorescence is higher. A valid relationship between fluorescence and the concentration of E3 bound NBD-K3 was therefore not possible. ${ }^{a}$ No membrane interacting recognition motifs like PNA oligomers, the usage of a fluorophore which is less influenced by the hydrophobicity of membranes and the application of a more effective technique to remove fluorescent probes (e.g. centrifugal concentrators) might help to overcome the drawbacks of the presented experimental setup for the determination of the degree of peptide insertion. ${ }^{174]}$

\footnotetext{
a Insertion tests based on fluorescent probes were performed by NINA SCHMIDT in the context of her Bachelor thesis.
} 


\subsection{5) Influence of Peptide Purity on Leaflet Mixing Experi- ments}

By analytical UPLC, purities of the used peptide samples were determined. For PNA-LR/TMD peptide hybrids as raw products, purity degrees of $15-40 \%$ were determined by the integration of chromatograms. For E3/K3 based model peptides, the determined purity degrees were only slightly higher (20-40\%, Appendix, Chromatography Results, Tables A-1 and A-2). It is assumed that impurities of the model peptide samples affect experimental outcomings of the performed leaflet mixing assays due to peptide fragments which associate with the model membranes and thereby inducing membrane destabilisation. As a first attempt to examine this assumption, E3/SybK and K3/SxE peptides were purified by HPLC. Collected probes had yields of $61 \%$ (not purified E3/SybK: $18 \%$ ) and $45 \%$ (not purified K3/SxE: $31 \%$ ). In TLM assays using raw products and peptides purified by HPLC, slightly different rates of leaflet mixing were observed (Figure 3.4.5-1). Admittedly, for both measurements of the combination $\mathrm{E} 3 / \mathrm{SybK}+\mathrm{K} 3 / \mathrm{S} x \mathrm{E}$ the increase of $\mathrm{NBD}$ emission due to leaflet mixing was relative low compared to other presented combinations, probably because of a low degree of peptide insertion, deviations from the optimal ratio of labelled and unlabelled liposomes of 1 to 4 (determination of lipid amounts of proteoliposome samples was not performed) and/or unstable labelled proteoliposomes. Nevertheless, raw products exhibited a higher increase of NBD emission after 20 min of detection time than the purified peptides.

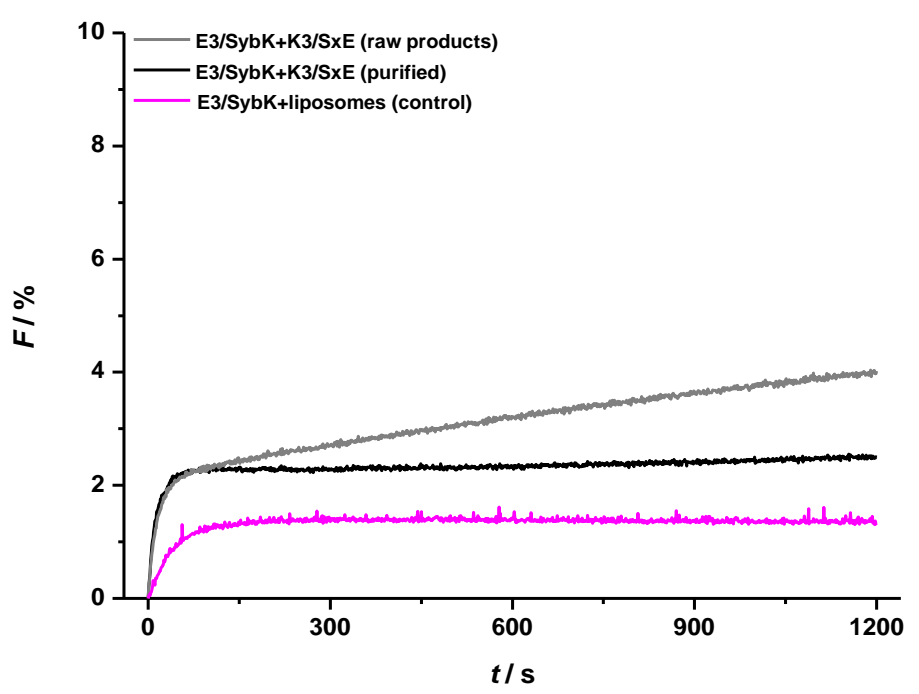

Figure 3.4.5-1 TLM experiments of labelled and unlabelled liposomes decorated with E3/SybK and K3/SxE peptides, respectively: Grey curve represents leaflet mixing of one TLM assay with not purified 
E3/SybK and K3/SxE peptides. Black curve represents leaflet mixing of one TLM assay with purified peptides. Magenta curve represents leaflet mixing of labelled E3/SybK liposomes with unlabelled liposomes and serves as control.

Strong fluorescence increases of both measurements within the first $75 \mathrm{~s}$ happened probably because of molecular recognition and accompanied leaflet mixing between the proteoliposome populations. Moreover, the increased hydrophobicity of the environment through the addition of unlabelled proteoliposomes support the initial fast fluorescence increase as well, because the emission of NBD is sensitive with regard to its environment. ${ }^{[173]}$ However, the subsequent slight increase for proteoliposomes with not purified peptides (grey curve in Figure 3.4.5-1) took probably place because of unspecific leaflet mixing and rupture processes of the labelled proteoliposomes which might be induced by higher degrees of impurity of the unpurified peptide samples. It must be noted that these comparing measurements concerning the influence of peptide purity on leaflet mixing assays were performed only once. Measurements with other peptide combinations exhibiting higher leaflet mixing rates and adjusted proteoliposome ratios as well as the control of peptide insertion must be performed to get a more exact view on the influence of sample impurities. 


\subsection{6) Size of Proteoliposomes and Its Influence on Fusion}

\subsubsection{1) Determination of Proteoliposome Size}

Since the 1970's, dynamic light scattering (DLS) is used to determine the size of liposomes. ${ }^{[175]}$ The sample is illuminated by laser light which is scattered by particles. Scattered light signals are detected in right angle $\left(90^{\circ}\right)$ or back angle $\left(\sim 175^{\circ}\right)$ position and depend on the Brownian motion of particles (Figure 3.4.6.1-1).

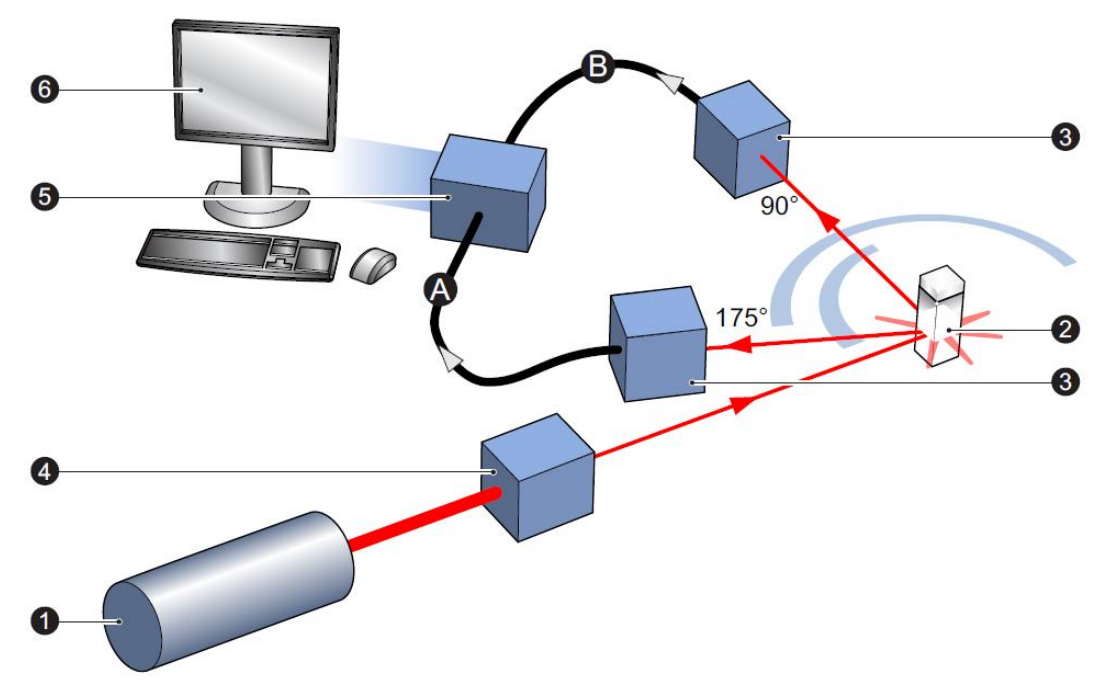

Figure 3.4.6.1-1 Experimental setup of dynamic light scattering measurements: Two common positions for the detector are possible, either $90^{\circ}$ or $\sim 175^{\circ}$ with respect to the sample (depends on device type). Laser (1), sample-cell (2), detectors (3), attenuator (4), correlator (5) and computer (6), (taken from ref. 176).

The usage of a back angle has several advantages: The laser beam does not travel through the entire sample and therefore less multiple scattering (additional scattering of already scattered light) occurs. Because of this, it is possible to measure sample at higher concentrations and the error resulting from dust particles is reduced (big dust particles tend to scatter in the forward direction). The $90^{\circ}$ arrangement reduces the detectable size range of particles due to a longer pathway through the sample cell. In both experimental setups scatter signals are interpreted as an autocorrelation function, which decay over time, if the signal at $t=0 \mu \mathrm{s}$ is balanced with the following signals of the measurement (correlation graphs in Figures 3.4.6.1-2 and 3.4.6.1-3). The decay constant is proportional to the translational diffusion coefficient $D_{\mathrm{t}}$. Through the 
STOKES-EINSTEIN equation, $D_{\mathrm{t}}$ is related to the hydrodynamic diameter $D_{\mathrm{h}}$ of the particles within a dispersion:

$$
D_{\mathrm{h}}=\frac{k_{\mathrm{B}} T}{3 \pi \eta D_{\mathrm{t}}}
$$

Thereby, $k_{\mathrm{B}}$ is the Boltzmann constant, $T$ the absolute temperature in Kelvin and $\eta$ the dynamic viscosity of the dispersion medium. In general, it can be noted that smaller particles move faster than bigger ones. For the validity of DLS measurements it is assumed that the particles are spheric. ${ }^{[90]}$ Advantages of this kind of liposome size determination are the non-invasive nature of measurements and the fast performance. A disadvantage is the detection of large ensembles of particles which results in relative imprecise intensity-weighted averages (z-averages) and lacking information about the liposome structure. For example, it is not possible to distinguish between uni- and mutilamellar liposomes. ${ }^{[176,177]} \mathrm{A}$ basic feature of liposomes prepared by extrusion concerning their size, defined as diameter of a spheric particle, is: there is no distinct size. Liposome suspensions rather exhibit a size distribution which covers several tens of nanometers. However, sizes are not distributed homogeneously, but as a Gaussian distribution with a predominated centre of size (main size). In the present study, extrusion was performed using polycarbonate membranes with a nominal pore size of $100 \mathrm{~nm}$. Determined size distributions (detection by back angle) typically include sizes bigger than $100 \mathrm{~nm}$, but also sizes smaller than $100 \mathrm{~nm}$. During extrusion, liposome tubes are formed which pinch off yielding liposomes of different sizes due to pore depth and lipid composition. ${ }^{[93]}$ In Figures 3.4.6.1-2 and 3.4.6.1-3 selections of DLS measurements of liposomes carrying PNA and E3/K3 based model peptides are presented. The measurements were performed several months after extrusion. Due to aggregation processes during storage, the determined sizes (weighted average) were bigger than $150 \mathrm{~nm}$ for pure liposomes (Figure 3.4.6.1-2, A) and liposomes carrying K3/SxE and E3/SybE peptides (Figure 3.4.6.1-3, B-C). 
A

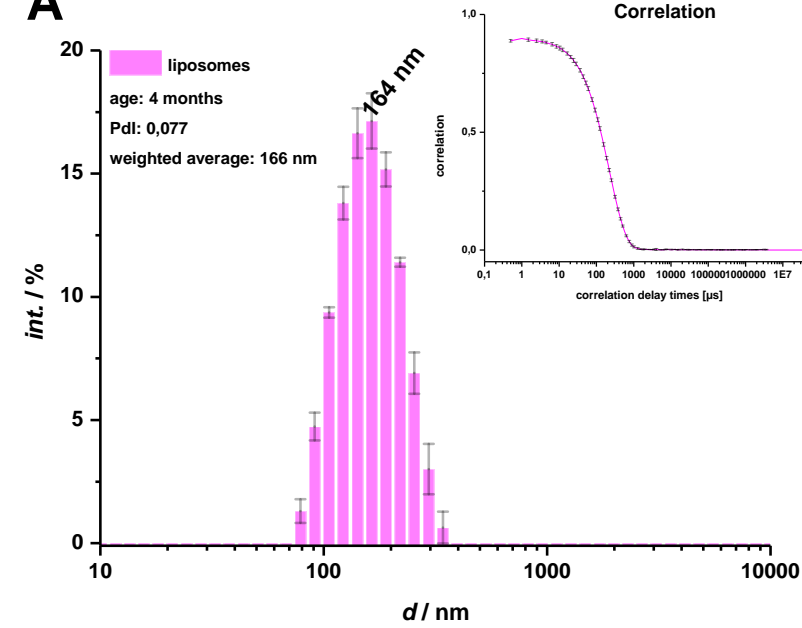

C

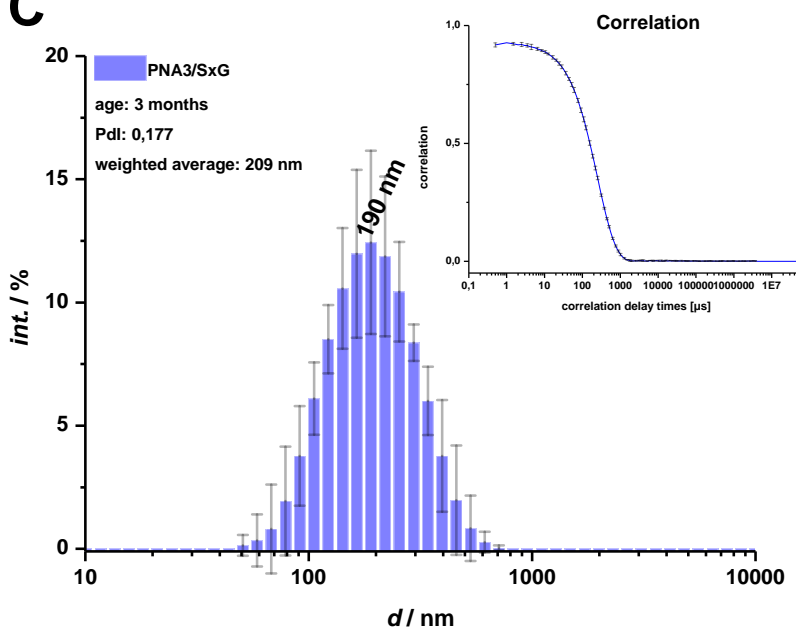

B

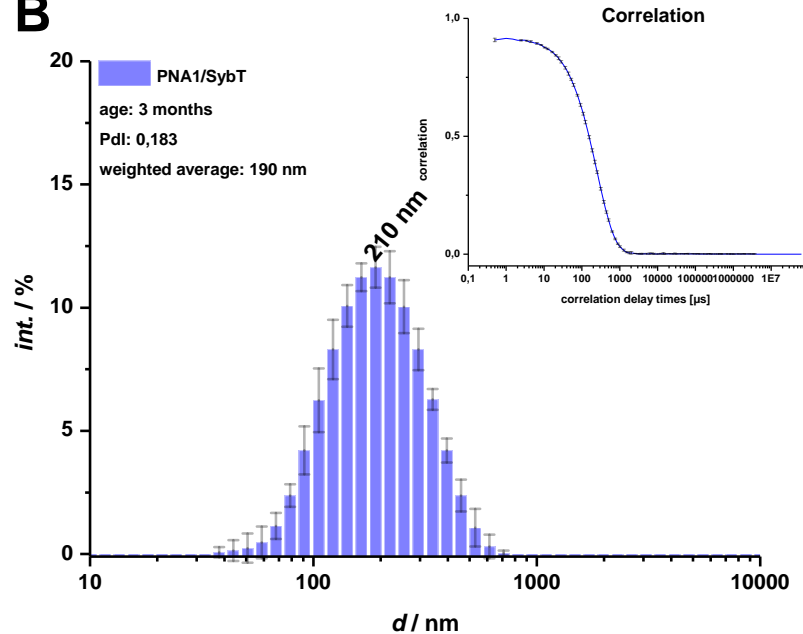

D

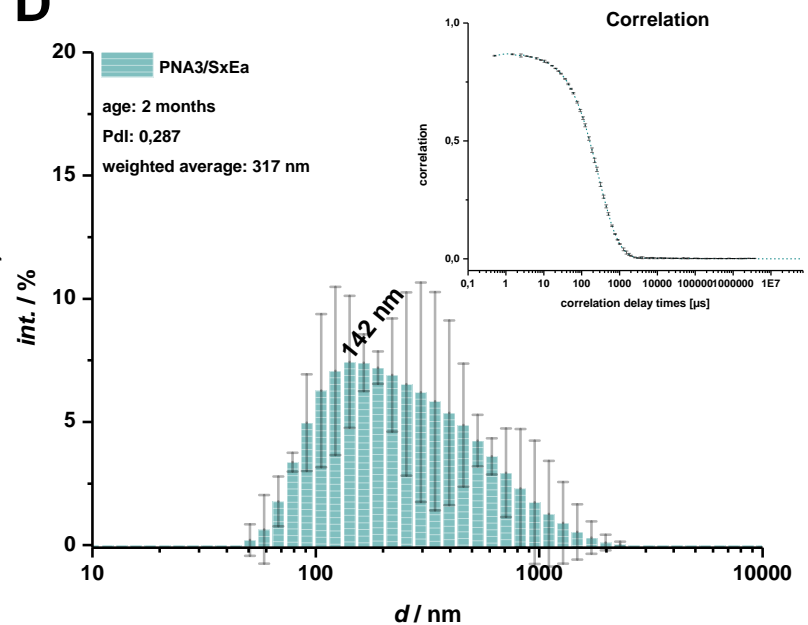

Figure 3.4.6.1-2 Selection of size distribution histograms with corresponding correlation curves of A) pure liposomes and liposomes decorated with B) PNA1/SybT, C) PNA3/SxG and D) PNA3/SxEa: All liposome populations were several months old when DLS measurements were performed. For each measurement two diameter values for the (proteo)-liposomes are given: the weighted average of size distribution and the main size at the top of highest bin of each histogram. The polydispersity index (Pdl) indicates the degree of polydispersity of the suspension (see text).

For proteoliposomes carrying PNA3/SxG and PNA3/SxEa, diameters were even bigger than $200 \mathrm{~nm}$ concerning the weighted average of size (Figure 3.4.6.1-2, C-D). PNA1/SybT liposomes with a size of $190 \mathrm{~nm}$ were only slightly smaller than $200 \mathrm{~nm}$ (Figure 3.4.6.1-2, B). E3/SybK liposomes with $137 \mathrm{~nm}$ were an exception (Figure 3.4.6.1-3, A). The smaller diameter is probably explainable by a shorter time range between proteoliposome preparation and DLS measurements (17 d). However, $\mathrm{K} 3 / \mathrm{S} x \mathrm{E}$ liposomes were stored over the same time before DLS measurements and exhibited a significant bigger diameter of $158 \mathrm{~nm}$ (Figure 3.4.6.1-3, B). It is known, that 
the $\mathrm{K} 3$ recognition motif interacts strongly via its lysine residues with membranes and thus might induce accumulation processes between proteoliposomes and therefore detection of bigger particles. ${ }^{[167]}$ In general, freshly prepared liposomes exhibit sizes closer to $100 \mathrm{~nm}$. In Tables A-6 and A-7 (Appendix, Determination of Proteoliposome Size) the sizes of additional samples are listed, determined by DLS and NTA measurements.

A

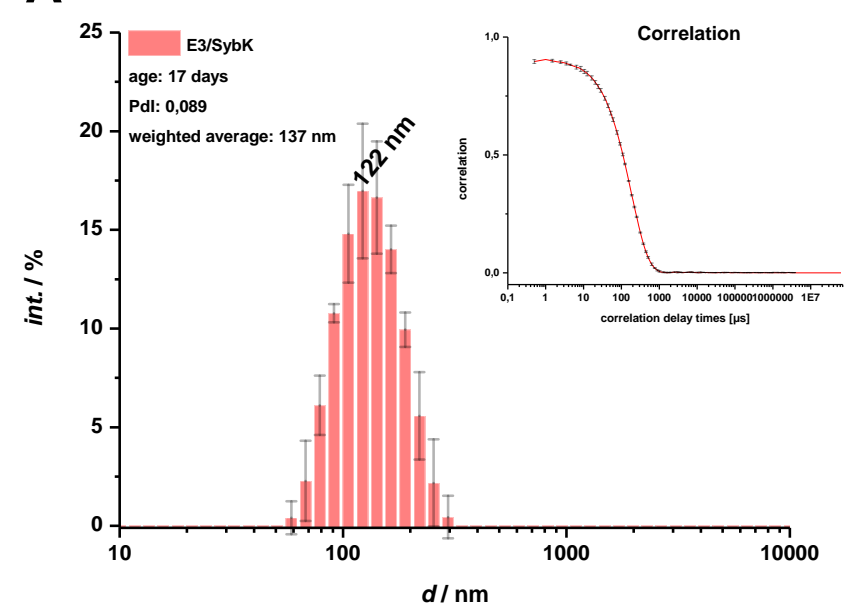

B

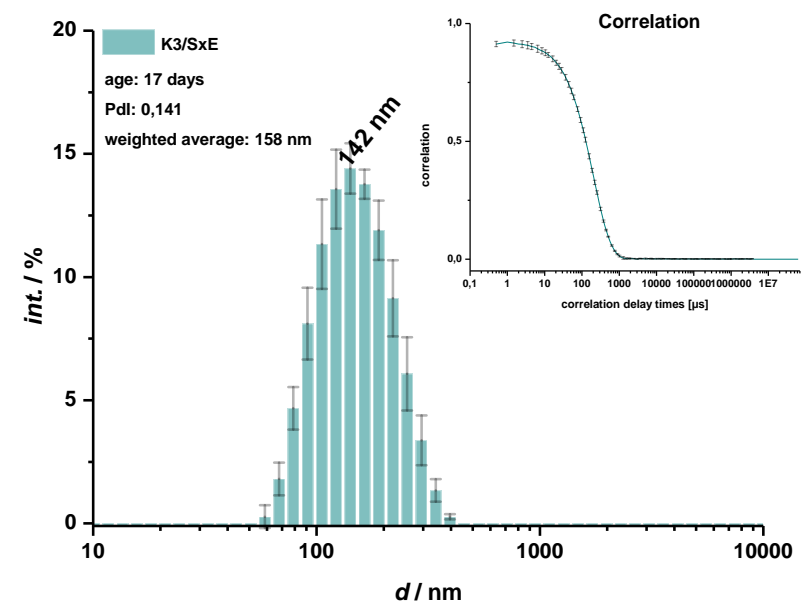

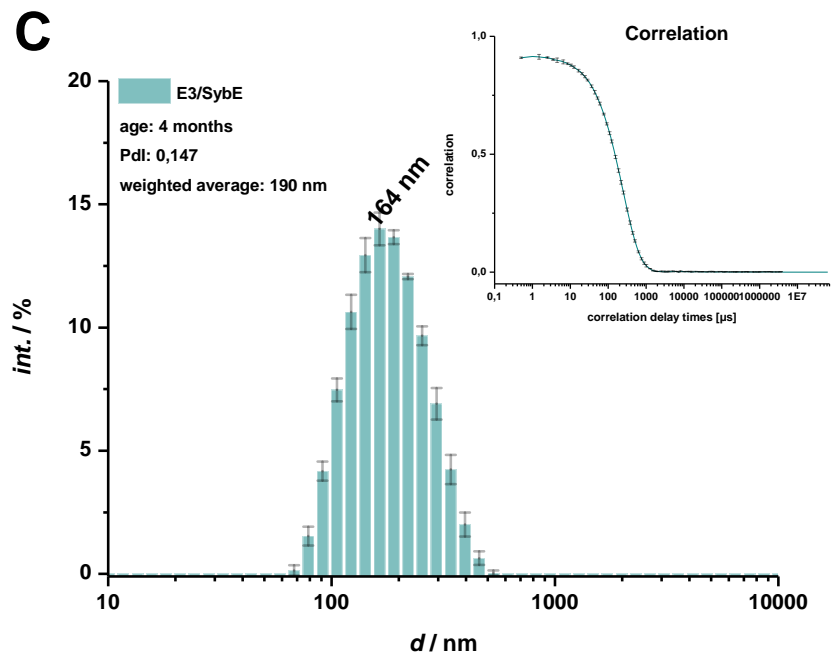

Figure 3.4.6.1-3 Selection of size distribution histograms with corresponding correlation curves of liposomes decorated with A) E3/SybK, B) K3/SxE and C) E3/SybE: For liposomes with E3/SybK and K3/SxE peptides DLS measurements were performed $17 \mathrm{~d}$ after preparation, whereas liposomes with E3/SybE were prepared 4 months before DLS measurements. For each measurement two values for the liposome diameter are given: the weighted average of size distribution and the main size in top of highest bin of each histogram. The polydispersity index (Pdl) indicates the degree of polydispersity of the suspension (see text). 
The proteoliposome samples, which were measured at the day of liposome preparation (designated by the term "few hours" in the Appendix) tend to have sizes below $150 \mathrm{~nm}$. LYGINA determined similar sizes for freshly prepared liposomes carrying PNA-LR/TMD peptide hybrids (111 nm for PNA1/SybT with a peptide/lipid ratio of 1:200). ${ }^{[170]} \mathrm{How}$ ever, MEYENBERG determined sizes of liposomes carrying E3/SybT (104 nm) and $\mathrm{K} 3 / \mathrm{S} \times \mathrm{G}(79 \mathrm{~nm})$ peptides which were smaller than the diameters determined in the present study. Reasons for it might be the smaller time range between preparation and DLS measurements as well as the lower peptide/lipid ratio of 1:1000.[178]

A further feature concerning the quality/stability of proteoliposome samples is the grade of dispersity expressed by the polydispersity index (Pdl): Suspensions exhibiting a Pdl smaller than 0.20 are defined as monodisperse. ${ }^{[178]}$ Nearly all proteoliposome populations (Figures 3.4.6.1-2 and 3.4.6.1-3) show values below 0.20 and therefore are considered to be monodisperse. Even a long interval between preparation and DLS measurements does not necessarily result in much higher polydispersity values of the suspensions (e.g. Figure 3.4.6.1-3, C), but in most cases a long-running storage is correlated to higher Pdl values. Pure liposomes exhibited the lowest measured Pdl of 0.077 in this study indicating a highly monodisperse suspension. Thus, the size distribution is relatively small compared to proteoliposome samples of similar age (Figure 3.4.6.1-2, A). On the other hand, liposomes decorated with PNA3/SxEa peptides have a Pdl of 0.287 indicative for a polydisperse suspension. The range of size distribution is broader than for the PNA3/SxG peptide carrying liposomes of similar age (Figure 3.4.6.1-2, C-D). In the case of PNA3/SxE liposomes, amounts of bigger liposomes exceed the amounts of smaller liposomes concerning the main fraction of the distribution. This result might be interpreted by stronger uncontrolled accumulation processes and indicates for a low quality/stability of this proteoliposome sample.

Another technique for size determination of liposomes is the nanoparticle tracking analysis (NTA) which is also based on the STOKES-EINSTEIN equation. However, here the light scattering signal is not analysed directly. Rather, light scattering is used to visualise liposomes. With the aid of a microscope, videos of several seconds are captured by a CCD camera. Subsequently, the video is analysed concerning the mean squared displacement of illuminated particles (Figure 3.4.6.1-4). 


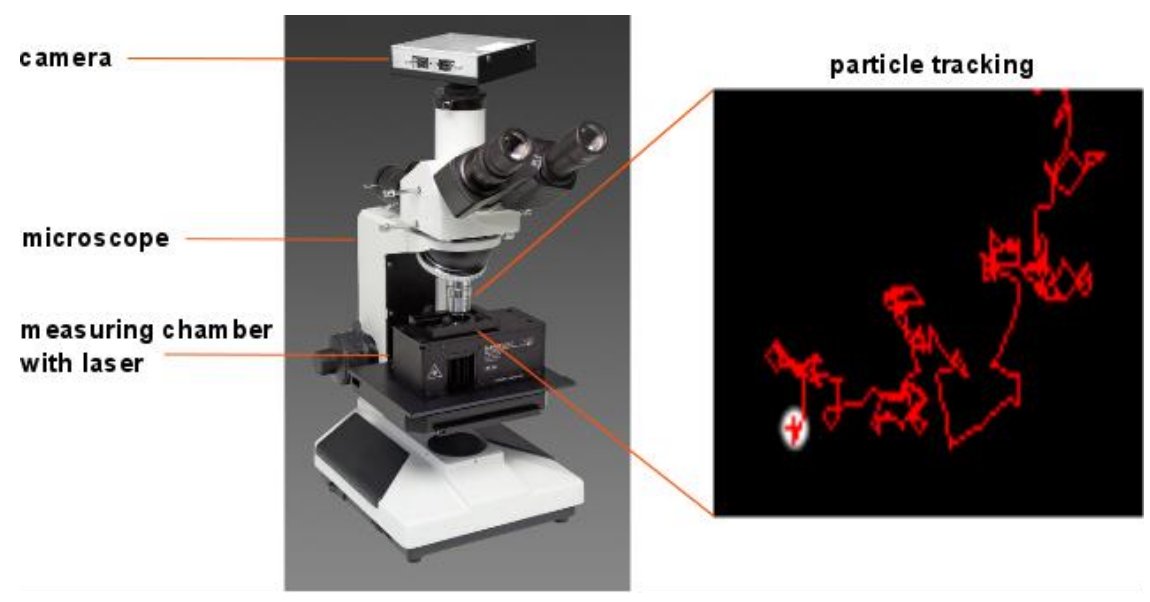

Figure 3.4.6.1-4 Experimental setup of the nanoparticle tracking analysis (left side) and an image of a typical particle track (right side), (taken from ref. 179).

The particle displacement is used to determine the translational diffusion coefficient and therefore the mean size (diameter) of the proteoliposomes. An advantage of NTA measurements compared to DLS, is the analysis of single particles. It is possible to estimate the concentrations of particles with certain diameters in the sample. Furthermore, it is possible to observe slow aggregation processes in real time. However, the concentration determination depends on the scattering volume which is on the other hand very small. Therefore, it is important to set the initial dilution in a range in which enough particles for a statistically significant analysis are present. An inappropriate high amount of particles leads to crossing particle trajectories (failed assignment of the particle to its trajectory) or to failures in particle distinction. Both affect the analysis of the diffusion coefficients of particles and thus the estimation of their number and size. ${ }^{[179]}$ Diameters of liposomes carrying E3/K3 based model peptides determined by NTA measurements are presented in the Appendix (Determination of Proteoliposome Size, Table A-7).

\subsubsection{2) Influence of Proteoliposome Size on Membrane Fusion}

It is obvious that suspensions contain proteoliposomes of different sizes, even if they are freshly prepared. Proteoliposomes of different sizes have different tendencies to fuse. Smaller proteoliposomes have a stronger tendency to fuse because of a higher bending stress of their membranes. This stress is relaxed by membrane fusion forming 
bigger proteoliposomes with less bending stress. ${ }^{[90]}$ Leaflet mixing assays applying the combinations $\mathrm{E} 3 / \mathrm{SybK}+\mathrm{K} 3 / \mathrm{SxK}$ or E3/SybTa + K3/SxGa with different sized liposomes revealed that coiled-coil based SNARE mimetics induce fusion when SUVs ${ }^{b}$ for both peptides were used. No fusion was observed when GUVs for K3-SxLR/TMD peptides and LUVs for E3-SybLR/TMD peptides were used. Considering the leaflet mixing assays with LUVs presented in Sections 3.1 and 3.2, it is concluded that E3/K3-LR/TMD peptide hybrids are capable to fuse liposomes with sizes in the range of 30-150 nm (SUVs and LUVs, respectively), but no LUVs ( $240 \mathrm{~nm})$ with GUVs (>1000 nm).

Furthermore, it was tested if LUVs carrying E3-SybLR/TMD peptides (E3/SybK and $\mathrm{E} 3 / \mathrm{SybTa}$ ) fuse with pore-spanning membranes (PSM) doped with $\mathrm{K} 3$ based model peptides (K3/SxK and K3/SxGa). In these experiments, only docking was observed. ${ }^{\mathrm{C}}$ $\mathrm{E} 4$ and $\mathrm{K} 4$ recogntion units connected by $P E G_{12}$ linkers and cholesterol anchors to LUVs and PSM, respectively, exhibit exclusively docking. However, native SNARE proteins (minimal fusion machinery) reconstituted in LUVs and PSM lead to membrane fusion. ${ }^{[19,88]}$ One possible reason for these observations might be $C$-terminal zippering of native SNARE proteins into the LRs/TMDs which is probably crucial to fuse relative stable membranes (i.e. membranes with little curvature). The tendency of liposomes carrying E4 or E3 recognition units anchored by cholesterol or native TMDs to dock at PSMs doped with $\mathrm{K} 4$ or K3 recognition units anchored also by cholesterol or native TMDs is probably explicable by the absence of $C$-terminal zippering. Thus, the herein used artificial coiled-coil recognition system might not induce further interactions between LRs and TMDs of Syb2 and Sx1A because of topological differences between both parts of the model peptides (Section 2.6.2.2 and the Appendix, Comparison of Native and Artificial Sequences).

Another interesting aspect of leaflet mixing assays using LUVs and PSMs was the rate of docking: SNARE mimetics with $C$-terminal lysines as carboxylic acids (E3/SybK and K3/SxK, zwitterionic $C$-termini) led to more docked LUVs than the corresponding peptides with native aas as amides (E3/SybTa and K3/SxGa, uncharged $C$-termini). This

\footnotetext{
b Prepared by the detergent removal method, peptide/lipid ratio of 1:250 and lipid composition of POPE/POPS/DOPC/cholesterol/lab. PE (19:10:50:20:1, $n / n / n / n / n)$.

c Leaflet mixing and PSM assays were performed by RAPHAEL HUBRICH, Institute of Organic and Biomolecular Chemistry, Georg-August University Göttingen.
} 
observation supports the assumption that $C$-terminal charges are crucial for the correct insertion of TMDs within the membrane (Section 3.4.2).

\section{5) Results of Atomic Force Microscopy}

\subsection{1) Detection of Membrane Fusion by Colloidal Probe Microscopy}

For colloidal probe microscopy (CPM) measurements, the LRs and TMDs of Syb2 and Sx1A were used, which carry glutamates (SybE and SxE) or the amides of threonine and glycine (SybTa and $\mathrm{SxGa}$ ) as $C$-terminal aas. As recognition units, PNA1 and PNA3 decamers (parallel recognition) are linked to the mentioned LRs/TMDs. Thefollowing combinations were tested: PNA1/SybE + PNA3/SxE and PNA1/SybTa + PNA3/SxGa. In general, CPM measurements confirmed interactions between the PNA recognition units, because the detected adhesion forces were roughly ten times higher in the presence of PNA-LR/TMD peptide hybrids than in the absence. The adhesion forces $f_{A}$ were determined by retraction curves (Figure 3.5.1-2). In addition, for model peptides with $C$-terminal glutamates and amide modified threonine/glycine, 2 and 7 fusion events (hemifusion and/or full fusion) out of 600 measurements were detected, respectively. Potential (hemi)-fusion was detected by breakthrough events in the approach curves (Figure 3.5.1-1). Both results are statistically not relevant to conclude for efficient fusion. Two events (2 out of 600, $0.3 \%$ ) determined for peptides with glutamate $C$-termini (PNA1/SybE + PNA3/SxE) are consistent with the FRET based bulk leaflet mixing measurements which exhibit no significant increase of donor emission (Figure 3.3.1-1). In contrast, for peptides with amide modified native aas (threonine and glycine), breakthrough events were also hardly observed by CPM (7 out of 600 , $1.2 \%$ ), although leaflet mixing assays indicate at least hemifusion. ${ }^{\mathrm{d}}$

\footnotetext{
${ }^{d}$ CPM measurements were performed by HANNES WITT, Institute of Physical Chemistry, Georg-August University Göttingen.
} 


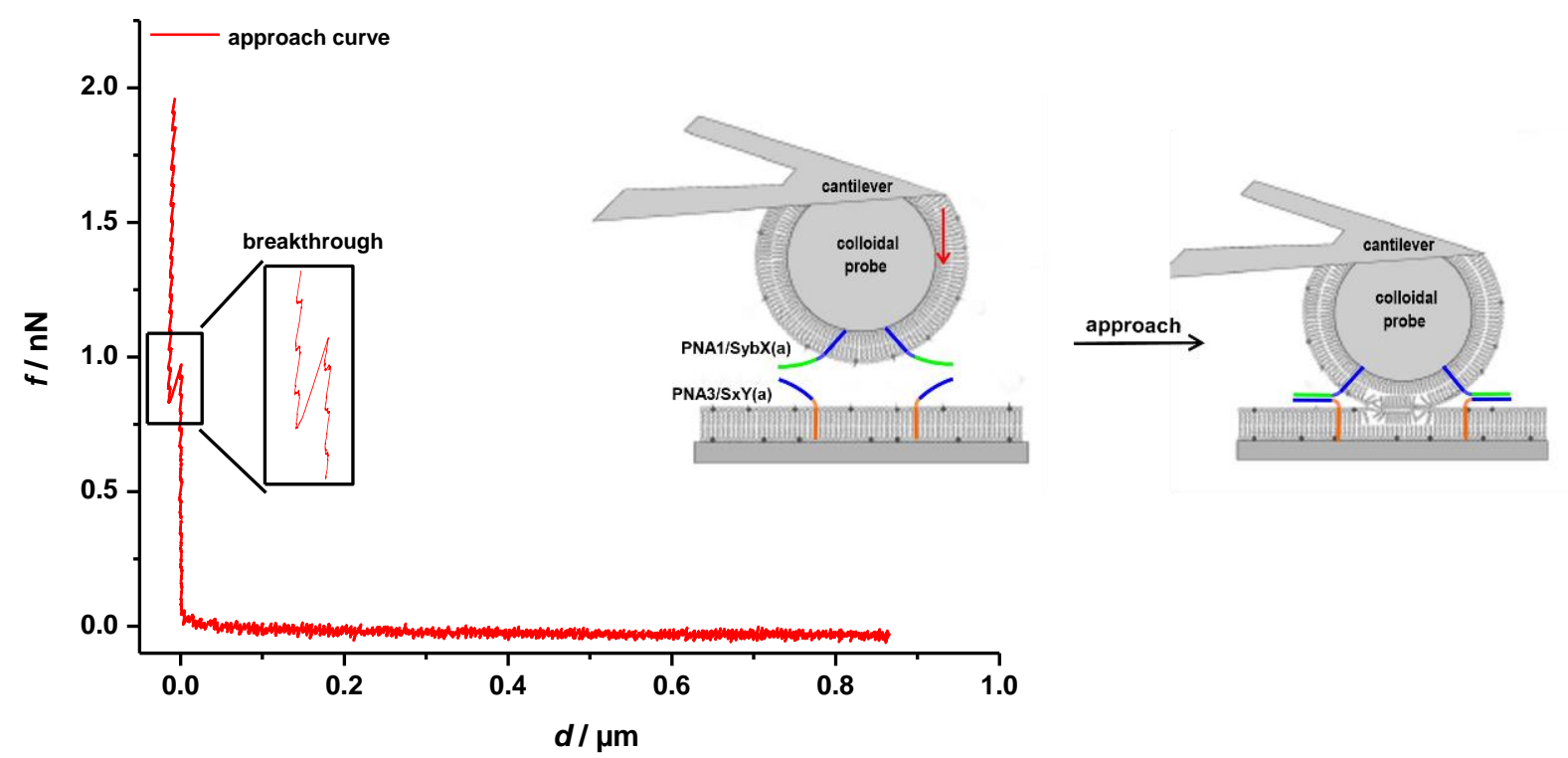

Figure 3.5.1-1 Exemplary approach curve (PNA1/SybTa + PNA3/SxGa) with the illustration of a breakthrough event. Window highlights the kink of a breakthrough event which is indicative for a (hemi-)fusion event (dwell time: $1 \mathrm{~s}$, trigger point: $1.0 \mathrm{nN}$ ), (illustration based on ref. 156).

Native SNARE proteins Syb2, Sx1A and SNAP 25 which were examined by OELKERS et al. under similar conditions (dwell time: $10 \mathrm{~s}$, trigger point: $0.2 \mathrm{nN}$ ) lead to considerable more fusion events (hemifusion: $16.2 \%$, full fusion: $7.6 \%$ ) which is explainable by the higher energy barrier for (hemi)-fusion of membranes at colloidal probes and solid supports. These membranes are less curved and thus less capable for membrane merger than SUVs or LUVs. The result is in line with pore spanning membrane (PSM) based measurements, in which also slightly curved membranes were applied (Section 3.4.6.2). ${ }^{[88,180]}$ The minimal SNARE fusion machinery is obviously more fusogenic than the PNA based model peptides. Due to experimental noise, the direct detection of (hemi)-fusion events through approach curves is not possible.

However, the formation of force plateaus during retraction is an additional indirect indicator for fusion events, because plateaus can be indicative for the formation of lipidic connections between the membranes of the spherical probe and the solid support (Figure 3.5.1-2). The simultaneous observation of breakthrough events in the attraction curve and force plateaus in the retraction curve might be a good indicator for fusion events. Nevertheless, due to low statistics of the performed CPM measurements it was not possible to observe both events simultaneously. 


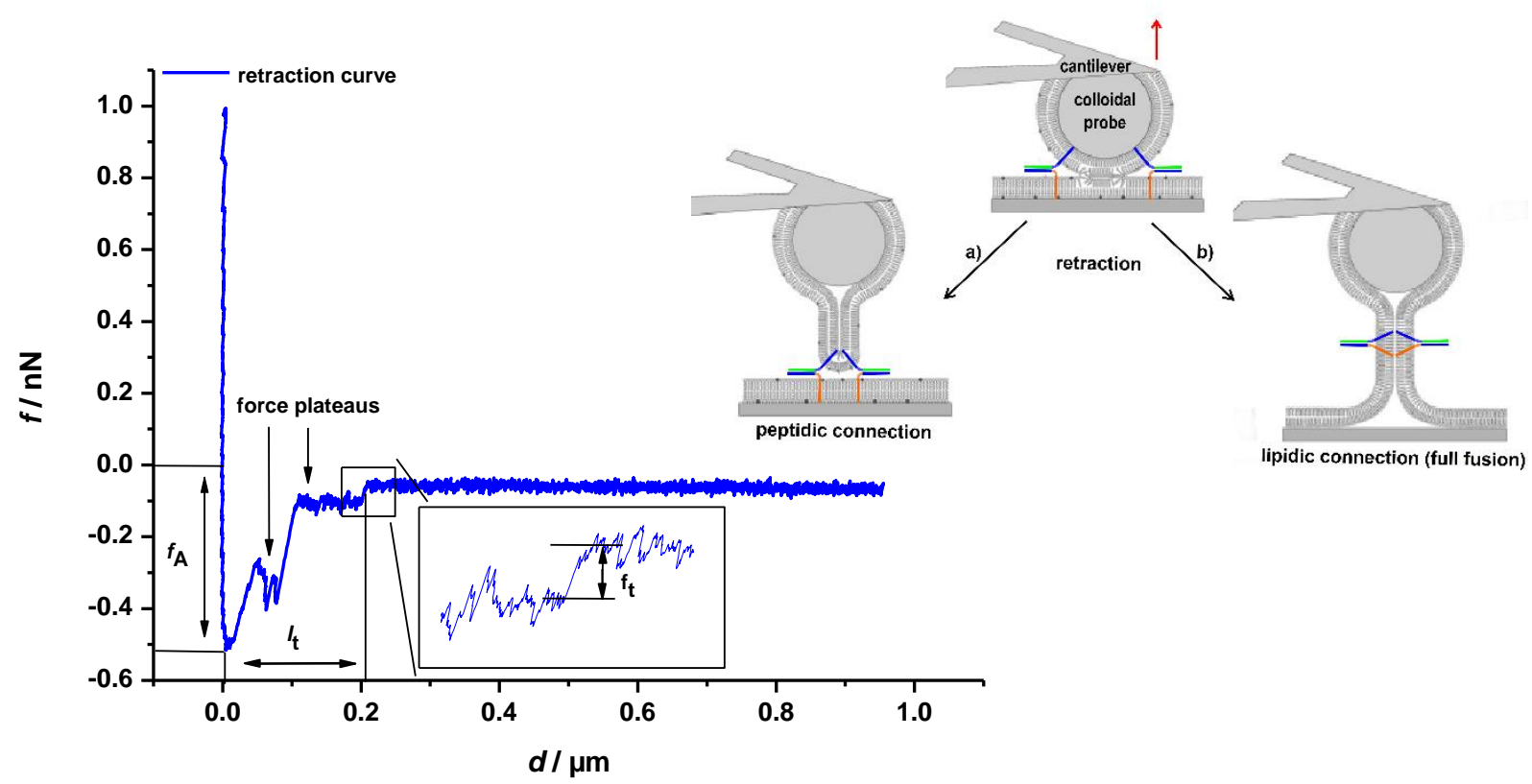

Figure 3.5.1-2 Exemplary retraction curve (PNA1/SybTa + PNA3/SxGa) with illustration of two possible connections between the membranes: a) Connection through the recognition motifs of the SNARE mimetics. The force plateau evolves due to detaching of the membrane from the colloidal probe. b) Connection through hemifused membranes. The force plateau evolves due to the separation of the membrane from the solid support. (right). Arrows highlight force plateaus of the exemplary retraction curve, which are indicative for peptidic or lipidic connections. The adhesion force $f_{\mathrm{A}}$ of the presented curve is $\sim 510 \mathrm{pN}$, the tether length $l_{\mathrm{t}}$ is $\sim 200 \mathrm{~nm}$ and the tether force $f_{\mathrm{t}}$ is $\sim 45 \mathrm{pN}$ (illustration based on ref. 156).

Furthermore, it must be noted that the force plateaus of the retraction curves can also evolve due to molecular connection between the SNARE mimetics without membrane fusion. However, retraction curves include additional information concerning membrane interactions and tether features: For instance, the adhesion force $f_{\mathrm{A}}$ can be used to determine the number of bonds in the contact zone. The tether length $t$ is directly observable through the retraction curves and the tether force $f t$ can be used for the determination of the tether radii.. ${ }^{[156,181]}$ In conclusion, a valid comparison between FRET based leaflet mixing results of Section 3.3 and the results of the CPM measurements is not possible because of the low statistics of the CPM measurements as well as the experimental uncertainties of the leaflet mixing results (influencing factors described in Section 3.4). However, possible reasons for the differences of the results determined by FRET based bulk leaflet mixing and CPM measurements might be allegeable by the prevalent curvature stress of the model membranes in both experimental setups: In bulk measurements, LUVs were used which are much stronger curved than the solid supported membranes of the CPM measurements. Moreover, for 
CPM measurements it is conceivable, that the solid support hinders membrane fusion because of a restricted mobility of the SNARE mimetics and interactions of the $C$-termini with the surface of the solid supports. ${ }^{[98]}$

\subsection{2) Energy Barrier for $C$-Terminal Peptide Immersion into Membranes}

The last step of SNARE mediated membrane fusion, i.e. pore formation, depends probably on the immersion of $C$-terminal ends of the TMDs of Syb2 and Sx1A into the membranes (Section 3.3). The movement of $C$-terminal ends into the headgroup region (bilayer interface) and hydrophobic core of the membranes might induce perturbation of the lipid continuity and thus the rearrangement of lipids ending in the openness of a fusion pore. ${ }^{[14]}$ WIMLEY et al. determined free energies for the transfer of proteinogenic aas from the bilayer interface to water by means of equilibrium dialysis and reverse-phase HPLC (determination via partition coefficients). ${ }^{[182]}$

Table 3.5.2-1 Free energy transfer from bilayer interface to water determined by WIMLEY et al. (values include side chain and backbone contribution), (taken from ref. 182).

\begin{tabular}{|l|c|c|}
\hline \multicolumn{1}{|c|}{ amino acid $(\mathrm{X})$} & $\Delta \mathrm{G}_{\mathrm{x}}\left[\mathrm{kcal} / \mathrm{mol}^{\mathrm{a}}\right.$ & $\Delta \mathbf{G}_{\mathbf{x}}[\mathrm{kcal} / \mathrm{mol}]^{\mathrm{b}}$ \\
\hline threonine & $-0.14 \pm 0.06$ & not given \\
\hline glycine & $-0.01 \pm 0.05$ & not given \\
\hline lysine & -0.44 & $-0.99 \pm 0.11$ \\
\hline glutamate & $-2.02 \pm 0.11$ & $0.01 \pm 0.05$ \\
\hline
\end{tabular}

Remarks: Experiments were performed at a) $\mathrm{pH}=8$ and $\mathbf{b}) \mathrm{pH}=2$. Thus, $C$-terminal carboxylic acid groups of the model peptide Ac-WL-X-LL-OH (X represents the position of examined aas) were either negatively charged (deprotonated state) or not charged (protonated state).

Table 3.5.2-1 presents the energy values for the $C$-terminal aas applied in the present study. The energy barrier for the transfer of glutamate at $\mathrm{pH}=8$ from water to the bilayer interface was the highest of the WIMELY study $(2.02 \mathrm{kcal} / \mathrm{mol})$ due to repulsive forces between the negative charges of the lipidic phosphodiester groups and the negative charges of the two carboxylic acid groups of glutamates. Therefore, it is assumed that $C$-terminal glutamates in model peptides induce a high energy barrier for the tran- 
sition of the $C$-termini of SNARE mimetics into the membranes. Indeed, SNARE mimetics with $C$-terminal glutamates as carboxylic acids induce no (as PNA-LR/TMD peptide hybrids) or only slight (as E3/K3-LR/TMD peptide hybrids) leaflet mixing in FRET based bulk measurments. However, energy values determined by WIMELY et al. do not represent the conditions applied in leaflet mixing experiments of the present study. For instance, LUVs were exclusively prepared by POPC. Moreover, pentapeptides were used and the examined aas were positioned in the middle of these peptides surrounded by leucines. This is not the peptidic environment of the applied $C$-terminal aas of the SNARE mimetics and experimental outcomings of the leaflet mixing experiments are not throughoutly explainable by the energy values given in Table 3.5.2-1. For example, $C$-terminal lysines have a higher energy barrier $(0.44 \mathrm{kcal} / \mathrm{mol})$ than the native aas threonine $(0.14 \mathrm{kcal} / \mathrm{mol}$ and glycine $(0.01 \mathrm{kcal} / \mathrm{mol})$, but the lysine combinations in the parallel recognition mode were the stronger fusogenes in FRET based leaflet mixing assays.

AFM measurements can be used to determine forces required for the detachment of lipids and TMDs from the membranes. ${ }^{[183,184]}$ For this approach, thiol functionalised peptides embedded in solid supported membranes were bound to cantilever tips covered by gold. [185] When the cantilever was pulled away from the solid supported membrane, kinks in force-distance curves indicative for membrane-peptide interactions were detected (Figure 3.5.2-1). These events could be used to calculate forces required for the movement of the TMDs through the membrane. In a first attempt, E3/SybT peptides were extended by $N$-terminal cysteines yielding Cys-E3/SybT peptides. AFM measurements with Cys-E3/SybT peptides revealed several force events. In Figure 3.5.2-1, B one detected force event is exemplary presented. 
A

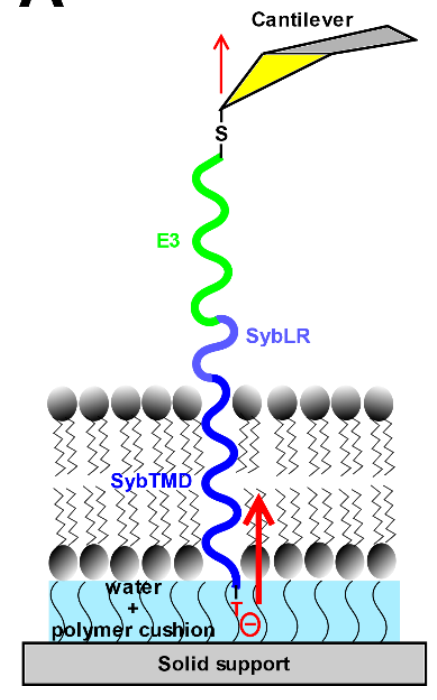

B

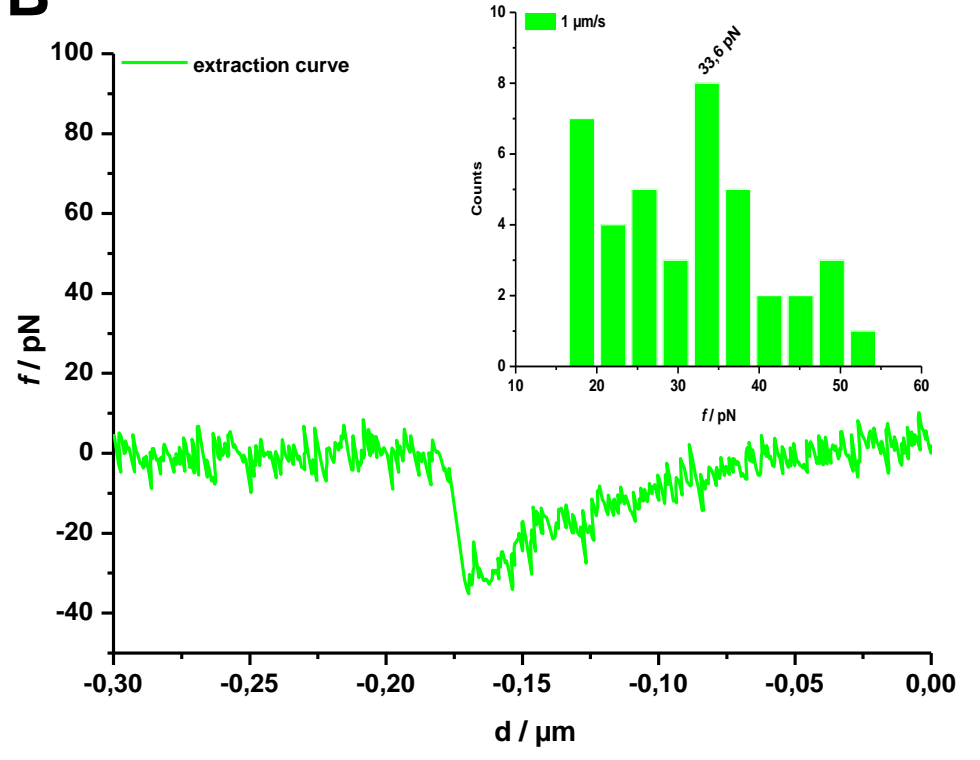

Figure 3.5.2-1 A) Illustration of AFM pulling experiment with E3/SybT-peptides. B) Exemplary force-distance curve. Three further types of force events were detected (not shown). Inlet: 40 events were recorded with a cantilever velocity of $1 \mu \mathrm{m} / \mathrm{s}$. Predominant force events were detected at $\sim 34 \mathrm{pN}$.

Concerning this kind of event, forces in the range of $30-40 \mathrm{pN}$ were detected $(45 \%$ of all events) and might corresponds to the removal of the model peptide from the bilayer. The removal depends among other factors (e.g. unfolding of the TMD helices) on the forces required for the detachment of the $C$-terminal end of the Syb2-TMD from the bilayer interface and the subsequent shift into the hydrophobic core of the membrane. ${ }^{[184]}$ However, more than the half of detected forces events were not in the range of $30-40 \mathrm{pN}$ and the broad distribution of force values prevent the application of a mathematic model (Figure 3.5.2-1, inlet of B). For valid results, it is necessary to perform more measurements with changing cantilever velocities. This might yield valid insights into force event characteristics and might allow the application of a suitable model (for instance, the BeLL-Evans model). ${ }^{[186,187]}$ If it is feasible, it would be possible to determine forces and related energies concerning the interactions between the $C$-terminal parts of integral SNARE mimetics and model membranes. ${ }^{e}$

\footnotetext{
e AFM measurements were performed by MARIAN VACHE, Institute of Physical Chemistry, Georg-August University of Göttingen.
} 


\section{6) Trigger for Peptide Mediated Membrane Fusion}

ZHENG et al. proposed that the inversion of E3 sequences (iE3 sequences) in the E3/K3 coiled-coil system leads to changes in the orientation and the oligomeric state of the coiled-coils. Instead of parallel dimers, antiparallel tetramers are formed (Section 3.1.2). ${ }^{[164]}$ Within the scope of this thesis, it was attempted to trigger molecular recognition and accompanied fusion of liposomes with iE3/SybT or K3/SxG peptides by the addition of dissolved K3 or iE3 peptides. (Figure 3.6-1).

A
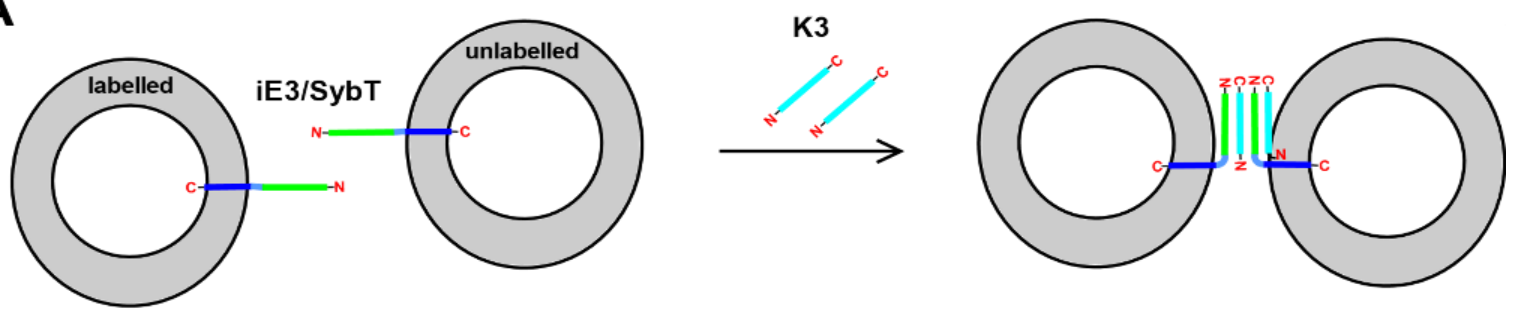

B
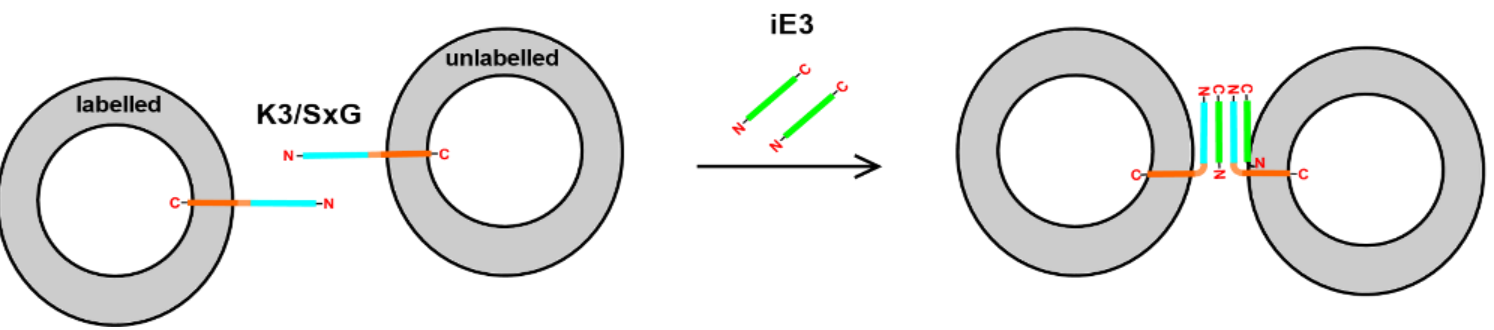

Figure 3.6-1 Illustration of the assumed mode of liposome docking induced by the addition of soluble components $(K 3, i E 3)$ to liposomes carrying $i E 3 / S y b T$ or $K 3 / S x G$ peptides. Subsequent membrane fusion is not shown: A) Liposomes are decorated with iE3/SybT peptides and K3 recognition units are added as dissolved components. B) Liposomes are decorated with K3/SxG SNARE mimetics and iE3 peptides are added as dissolved components.

However, when dissolved K3 peptides were added to membrane anchored iE3/SybT SNARE mimetics, no significant increase of donor emission was detected. Admittedly, a slight increase of NBD emission was observed, when unlabelled iE3/SybT liposomes were added to labelled iE3/SybT liposomes. The increased hydrophobicity of the medium through the addition of unlabelled proteoliposomes might amplify NBD emission within the first 120 seconds. ${ }^{[173]}$ After stabilisation of the NBD signal, different amounts of dissolved K3 peptides were added, but the additions did not lead to enhanced leaflet mixing and thus not to membrane fusion. Drops of emission during the measurements 
with $30 \mathrm{nmol}$ and $60 \mathrm{nmol}$ are consequences of the increased volumes resulting from the added K3 peptide solutions (Figure 3.6-2, A). The suggested tetramer formation seems to be hindered by the raised restriction of membrane anchored iE3 recognition units compared to the tetramer formation of $\mathrm{iE} 3$ and $\mathrm{K} 3$ peptides in solution without TMDs. Furthermore, iE3-K3 interactions compete with membrane-K3 and K3-K3 interactions. Therefore, it is conceivable that $\mathrm{K} 3$ peptides bind to membranes or other dissolved K3 units and are excluded from tetramer formation. ${ }^{[167,188]}$
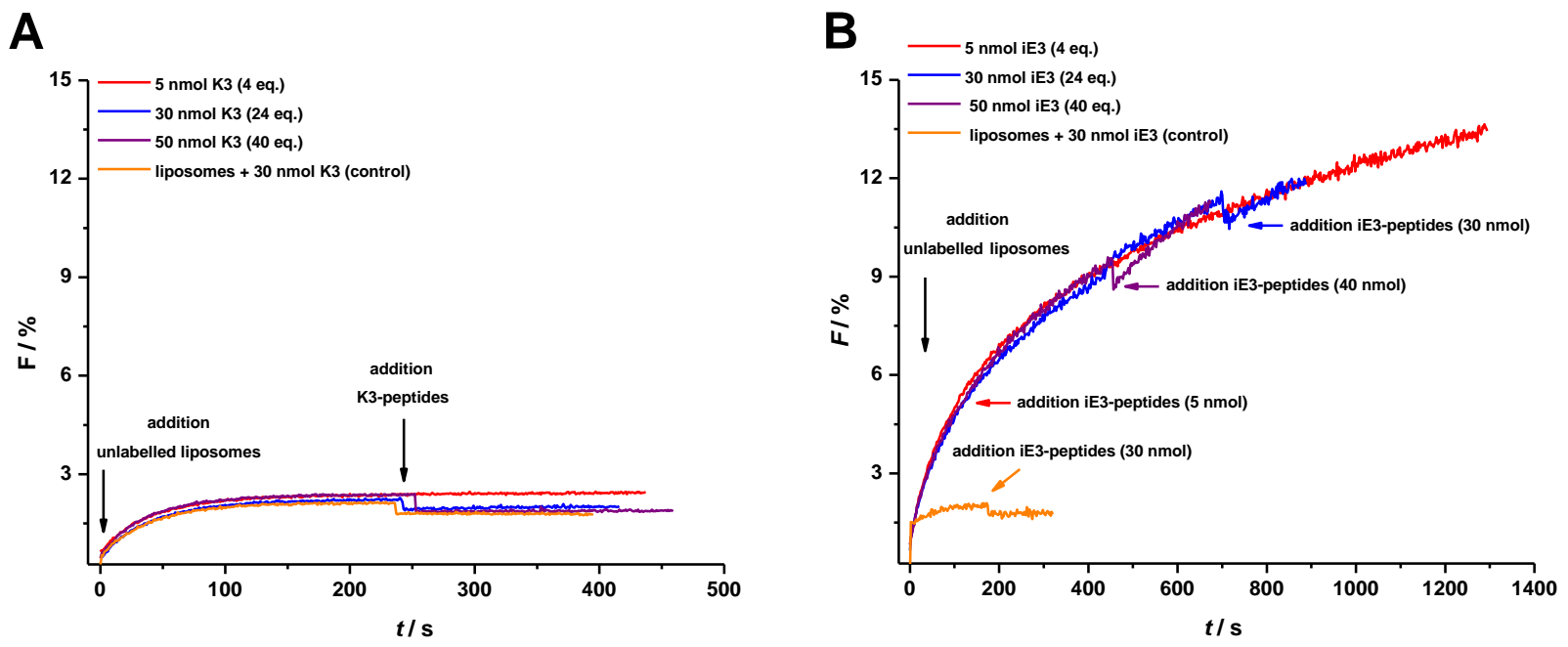

Figure 3.6-2 Total leaflet mixing assays of labelled and unlabelled proteoliposomes doped with $1.25 \mathrm{nmol}$ of $\mathbf{A}$ ) $\mathrm{iE} 3 / \mathrm{SybT}$ or $\mathbf{B}) \mathrm{K} 3 / \mathrm{SxG}$ model peptides. In both experiments no membrane fusion was observed due to the addition of $\mathbf{A}$ ) dissolved K3 or B) iE3 recognition units.

When dissolved iE3 peptides were added to membrane anchored K3/SxG SNARE mimetics, also no leaflet mixing amplifying effects of the dissolved components were detected (Figure 3.6-2, B). However, after the addition of unlabelled K3/SxG liposomes, continuous increases of NBD emission indicative for leaflet mixing were observed. This is explicable by the homodimerisation of K3/SxG SNARE mimetics which reside in opposing membranes. Additionally, the high affinity of anchored K3 recognition units to membranes might induce membrane destabilisation and intermembrane contacts through binding events to apposed membranes. Both kinds of interactions might support leaflet mixing. ${ }^{[167,188]}$ The addition of dissolved iE3 peptides did not amplify the 
increase of NBD emission. Thus, it is assumed that no fusion triggering tetramer formation occurs. ${ }^{\dagger}$

${ }^{f}$ Experiments for the examination of membrane fusion triggering effects of dissolved peptidic components were performed by NINA SCHMIDT in the context of her Bachelor thesis at the Institute of Organic and Biomolecular Chemistry, Georg-August University Göttingen. 


\section{4) Conclusion and Outlook}

In general, parallel recognising SNARE mimetics are more fusogenic than mimetics in the antiparallel recognition mode. This is in line with the known predominant parallel recognition of the native SNARE proteins. ${ }^{[3]}$ It is obvious that parallel interacting model peptides are able to exert more force via their TMDs on membranes than in the antiparallel binding mode (Figure 2.7-2). Antiparallel recognition leads rather to docking than to hemifusion or full fusion. However, antiparallel interacting iE3/SybT and K3/SxG peptides induce hemifusion and partially full fusion. This is intriguingly, because the distance between the membranes connected by an antiparallel tetrameric coiled coil is estimated to be $\sim 6 \mathrm{~nm}$, but membrane fusion is induced at an intermembrane distance of $0.9 \mathrm{~nm}$ (Section 3.1.2). ${ }^{[45,164]}$ Therefore, other membrane fusion supporting factors must be present in this system. It is known that K3 peptides interact strongly with membranes via their lysine residues. ${ }^{[166]}$ Thereby, the membranes are shaped and probably destabilised. ${ }^{[167]}$ Furthermore, it is conceivable that the LRs of the model peptides are kinked between the docked membranes, because these regions are probably not $\alpha$-helical and thus rather flexible. The distance between the docked proteoliposomes might be smaller than $6 \mathrm{~nm}$. In addition, the tetramer formed by two iE3 and K3 units, respectively, might interact by its lysine residues on the flanks with the membranes. Peptidic connections via tetramer formation, membrane shaping through K3 units and interactions of K3 units with apposed membranes are sufficient to induce fusion.

The distance between membranes docked by antiparallel interacting PNA based model peptides is estimated to be $\sim 6 \mathrm{~nm}$ as well (Section 3.1.1). ${ }^{[113]}$ For PNA recognition units, no interactions with membranes are known. Therefore, no membrane fusion supporting interactions are available as in the case of $\mathrm{IE} / \mathrm{K} 3-\mathrm{LR} / \mathrm{TMD}$ peptide hybrids. This is reflected by PNA1/SybT and PNA2/SxG peptides, which exhibit less leaflet mixing than model peptides with iE3 and K3 recognition units (compare Figure 3.1.1-1, A with Figure 3.1.2-1, A). Generally, leaflet mixing of antiparallel interacting model peptides is probably rather unspecific than related to the typical membrane fusion mechanism (Figure 2.4-1). Antiparallel interacting PNA-LR/TMD peptide hybrids with $C$-terminal amides are the least fusogenic group of the SNARE mimetics tested in the pre- 
sent study. This is explicable with the reduced degree of peptide insertion due to lacking $C$-terminal negative charges and therefore, a decreased number of recognition sites. Moreover, membrane destabilisation through not inserted peptides leads to proteoliposome aggregation which exacerbates the detection of leaflet mixing by fluorescence (Sections 3.3.2 and 3.4.2).

Parallel coiled-coil formation of E3/K3 based model peptides induces generally stronger leaflet mixing than parallel PNA duplex formation. The energy which is released by $E 3 / K 3$ assembly is higher than for PNA1/PNA3 duplex formation $\left(T_{\text {melt }}(E 3 / \mathrm{K} 3)=57^{\circ} \mathrm{C}, \mathrm{T}_{\text {melt }}(\mathrm{PNA} 1 / \mathrm{PNA} 3)=46^{\circ} \mathrm{C}\right) .{ }^{[15,164]}$ In addition, the released energy is probably more effectively transferred to membranes via the TMDs, because the coiled-coil formation resembles native SNARE recognition more than the formation of parallel PNA duplexes (Sections 2.6.2.1 and 2.6.2.2). The $C$-terminal ends of the E3/K3-LRTMD peptide hybrids might exert more force on the membranes to rearrange lipids for fusion, because the movement of the TMDs towards the hydrophobic cores of the membranes is more distinct. ${ }^{[14]}$

Concerning the effects of $C$-terminal charges induced by the exchange of threonine (Syb2) and glycine ( $\mathrm{S} \times 1 \mathrm{~A})$ through lysine or glutamate on membrane fusion, it is concluded that negative charges have an inhibitory influence on membrane fusion. SNARE mimetics with $C$-terminal glutamates as carboxylic acids (two negative charges) lead to less leaflet mixing than the naturally occuring aas threonine and glycine (one negative charge, respectively) and lysines (zwitterionic) (Sections 3.3.1 and 3.3.3). The energy barrier for the transition of a twofold negative charged glutamate from the aqueous medium at the membrane surface into the membranes is higher than for onefold negative charged threonine and glycine as well as for zwitterionic lysine (Section 3.5.2).[182] Although the energy barrier for the transition of lysine as carboxylic acid into the membranes is higher than for native aas, model peptides with $C$-terminal lysine exhibit in most cases stronger membrane fusion (Sections 3.3.1 and 3.1). For lysines, it is suggested that these aas interact strongly with membranes and thereby destabilise them. The positive charge of the protonated amino group of the side chain interacts with the phosphates of the lipids and the residual hydrocarbon chain with the lipid tails (snorkelling interaction). ${ }^{[166]}$ Intriguingly, model peptide combinations with $C$-terminal glutamates for Syb-TMDs and lysines for Sx-TMDs are the most fusogenic in the present study. This is contradictory, because glutamates hamper fusion due to 
a high transition energy for the immersion of $C$-terminal ends into the membranes. ${ }^{[17]}$ Initially, it was expected that $C$-terminal lysines for both TMDs would be the most fusogenic combinations because of the strong membrane destabilising intractions of lysine. However, the higher leaflet mixing rates for combined $C$-terminal glutamates and lysines is rather explicable by the experimental setup of the leaflet mixing experiments: Liposomes carrying model peptides with $C$-terminal glutamates are more stable than liposomes doped with lysine modified peptides (both as carboxylic acids and as amides). Model peptides based on Syb2 always reside in labelled and Sx1 A based mimetics in unlabelled proetoliposomes. Thus, for glutamate/lysine combinations stable labelled and unstable unlabelled proteoliposomes are present. This is advantageous for leaflet mixing detection, because labelled proteoliposomes do not tend to aggregate. Consequently, the dilution of labelled membranes through unlabelled lipids and the decrease of the FRET effect is not hampered. Moreover, unlabelled proteoliposomes are prone for leaflet mixing processes with stable labelled proetoliposomes due to their instability. Tests with $C$-terminal lysines for Syb-TMDs (unstable labelled proteoliposomes) and glutamates for Sx-TMDs (stable unlabelled proteoliposomes) resulted in much less leaflet mixing (Figure 3.4.5-1). This underlines the assumption of hampered leaflet mixing detection through aggregation, because aggregated labelled proteoliposomes do not mix efficiently with unlabelled liposomes due to an adverse surface/volume ratio, i.e. labelled lipids of the interior of aggregates cannot participate in leaflet mixing. On the other hand, if unlabelled proteoliposomes aggregate, the dilution of labelled proteoliposomes can occur at the surface of the unlabelled aggregates.

E3/K3-LR/TMD peptide hybrids as $C$-terminal amides are the most fusogenic group in this study. The lacking $C$-terminal negative charges of carboxylic acid groups reduce the energy barrier for the transition of the $C$-termini from the aqueous environment into the membranes. Furthermore, E3/K3 recognition exert more force on membranes than the PNA duplex formation. Flawed insertion of model peptides with $C$-terminal amides might also support membrane fusion, because not inserted peptides adsorb at the surface of the membranes and destabilise them. However, for PNA based model peptides, the membrane fusion supporting effect of $C$-terminal amides is less distinct (not all amide combinations show stronger fusion than the carboxylic acid combinations, Figure 3.2-2). This is lesser explainable by structural features of the model 
peptides, but rather by the increased instability and accompanied tendency to aggregate of the labelled proteoliposomes. Especially lysine modified PNA-LR/TMD peptide hybrids yield unstable proteoliposomes (Section 3.4.2). Thus, the aggregation exacerbates the detection of leaflet mixing and hampers additionally molecular recognition (Section 3.2).

Lysine modified SNARE mimetics might support membrane fusion also by the presence of $C$-terminal positive and negative charges. RISSELADA et al. suggested by means of computer simulations that attractive forces between the $C$-termini of Sx-TMDs and Syb-TMDs enable a thinning-widening mechanism for membrane fusion (Figure 2.7-2). ${ }^{[13]}$ However, relative strong membrane fusion is induced for both parallel and antiparallel orientation (PNA-LR/TMD peptide hybrids). Therefore, it is not decent to conclude by performed leaflet mixing assays that attractions between $C$-terminal lysines support membrane fusion. It is rather reasonable to suggest that the already mentioned membrane destabilising effects of $C$-terminal lysines amplify leaflet mixing rates. To shed more light on this issue of SNARE mediated membrane fusion, it is necessary to use mixtures of SNARE mimetics with opposing $C$-terminal charges for one proteoliposome population, e.g. PNA1/SybKa and PNA1/SybEa for labelled liposomes as well as PNA3/SxKa and PNA3/SxEa for unlabelled liposomes (parallel recognition). The comparison of the same mixtures of modified TMDs in the antiparallel recognition mode (PNA1/SybKa and PNA1/SybEa for labelled liposomes as well as PNA2/SxKa and PNA2/SxEa for unlabelled liposomes) might clarify if a thinning-widening mechanism supports membrane fusion.

E3/K3-LR/TMD peptide hybrids exhibit only docking when reconstituted in membranes of slight curvature (GUVs and PSM, Section 3.4.6.2). Moreover, no significant membrane fusion is detected when PNA based model peptides are tested by CPM applying plane solid supported membranes (Section 3.5.1). However, when native SNAREs (Syb2, Sx1A and SNAP 25) are tested under similar conditions efficient membrane fusion was observed. [88,180] Thus, both applied SNARE mimetics (PNA and E3/K3 based) are less fusogenic than reconstituted native SNAREs. Less fusogenicity might be explicable by the absence of $C$-terminal zippering of the SNARE mimetics. Topological differences between the recognition units (PNA and E3/K3) and LRs of Syb2 and Sx1A might prevent zippering into the TMDs (Sections 2.6.2.1 and 2.6.2.2). Consequently, less force is exerted on the membranes and the perturbation of membrane 
continuity, which is important for fusion, is less distinct. Although SNARE mimetics based on coiled-coil forming peptides (E3 and K3) match structural features of native SNARE proteins much better than PNA recognition motifs, there are also differences in the interface distances of native and artificial recognition motifs. Furthermore, there are differences in structural transitions between the recognition units and LRs regarding the heptad registers of the contributing coiled-coil motifs (Appendix, Comparison of Native and Artificial Sequences, Figure A-8). An alternative to overcome topological problems regarding the transitions of recognition units and LRs might be the deletion of arginine (SybLR) and the addition of tyrosine (SxLR). Through these modifications, the aas of the LRs proposed for zippering interactions have the same distances to artificial recognition motifs than to the native SNARE motifs. ${ }^{[10]}$ Structural conditions for $C$-terminal zippering might be more appropriate for SNARE mimetics which are modified in this way (Figure 4-1).
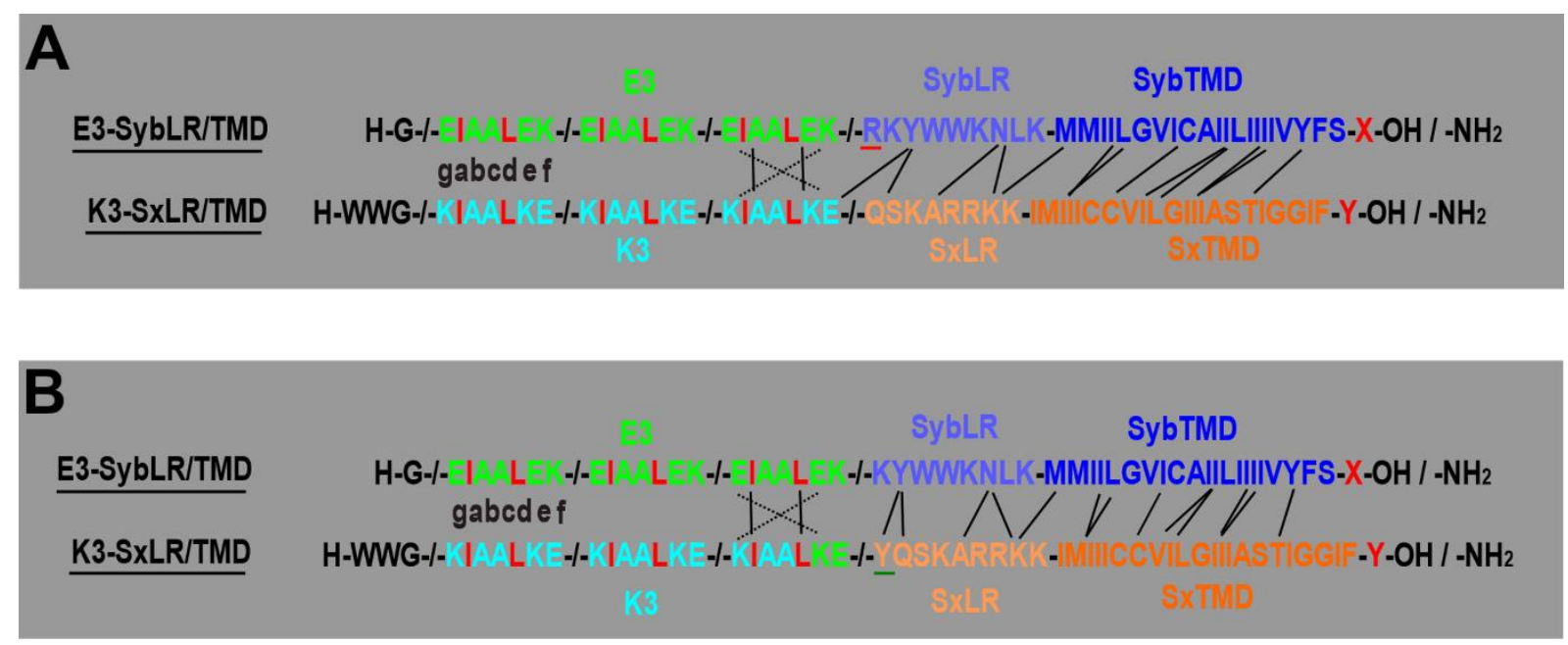

Figure 4-1 SNARE mimetics with coiled-coil forming peptides as recognition motifs: A) Sequences of SNARE mimetics applied in the present study. Possible deletion of arginine is underlined in red. B) Adjusted sequences concerning the distances between recognition units and LRs. Possible addition of tyrosine is underlined in green. Interacting aas of the LRs (indicated by black solid lines) are separated from the recognition units by the same number of aas like in native SNARE proteins.

Before mentioned results are mainly based on bulk leaflet mixing assays. In general, these assays have crucial disadvantages regarding assays which detect membrane fusion on a single event level. Aggregation and rupture of liposomes influence experimental outcomings. Additionally, average kinetics are detected and the distinction of fusion intermediates by TLM, ILM and content mixing is rather indirect and also suffer 
from average kinetics, aggregation, rupture and leakage. ${ }^{[88]}$ Therefore, the extended application of membrane fusion assays enabling the detection of single fusion events for herein examined model peptides might help to gain a closer insight into the role of TMDs in membrane fusion. Two possible assays for the detection of single fusion events base on pore-spanning membranes (PSMs) and colloidal probe microscopy (CPM) (Sections 2.6.3.4 and 2.6.1).

The purity of the applied SNARE mimetics is another crucial aspect in the present study. Synthesised peptides were used as crude products with purities in the range of 15-40 \% (Appendix, Chromatography Results, Tables A-1 and A-2). Peptide fragments in crude products might interact with the membranes of proteoliposomes. Peptide-membrane interactions might perturb membrane continuity and therefore, support leaflet mixing. Thus, it is problematic to confine differences between tested model peptide combinations solely on $C$-terminal modifications. Impurities have probably influence on experimental outcomings either. Higher purity degrees would reduce these experimental uncertainties (Section 3.4.5).

Another important aspect of membrane fusion in artificial systems is the degree of peptide insertion. For instance, different procedures of peptide insertion might lead to different degrees of insertion. Reconstitution protocols must be controlled concerning their ability to insert integral model peptides. Moreover, it must be ensured that different SNARE mimetics insert at the same level. Otherwise, the comparison of different peptide combinations regarding structural modifications is not meaningful. Unfortunately, the determination of SNARE mimetics, which are available for molecular recognition between the proteoliposomes, was not successful in the present study (Section 3.4.4).

Concerning the proteoliposome preparation it is crucial to determine the loss of lipid material during extrusion and size exclusion chromatograpy, because losses differentiate between the individual formulations. As a consequence, different ratios of labelled and unlabelled proteoliposomes are applied which influenced experimental outcomings in the present study as well (Section 3.4.3). The usage of two labelled liposome samples would be advantageous, because the lipid loss could be easily controlled by means of fluorescence (Section 3.4.1). Time consuming phosphate determination would be avoided and the fluorescence increase due to unspecific liposome rupture could be circumvented. 


\section{5) Experimental Part}

\section{1) Solvents}

DMF, DCM and NMP were obtained from Merck, Fisher Scientific or Carl Roth. Acetonitrile and methanol were purchased from Fisher Scientific. All used solvents were of laboratory grade ( $\geq 99.8 \%$ ) or HPLC grade ( $\geq 99.99 \%$ ). Distilled water was filtered by the arium $\Theta$ mini ultrapure water system (Sartorius, Germany) prior to use.

\section{2) Reagents}

Preloaded Wang resins and the not preloaded Nova PEG Rink amide resin were purchased from Nova Biochem, Switzerland. The Rink amide MBHA resin (not preloaded) was obtained from GL Biochem (Schanghai) Ltd., China. Piperidine, DIPEA, acetic acid anhydride, HATU, HBTU, Oxyma and trifluoroacetic acid were ordered from Carl Roth, Germany. 2,6-lutidine from Acros Organics, USA, was used. HOAt, HOBt, amino acid building blocks were purchased from GL Biochem (Schanghai) Ltd., China. DIC was from Iris Biotech, Germany. PNA building blocks were obtained from ASM Research Chemicals, Germany. Lipids were acquired from Avanti Polar Lipids, USA. All reagents were of the highest grade available.

\section{3) General Methods}

\subsection{1) Lyophilisation}

Aqueous solutions of peptides were frozen using liquid nitrogen. Frozen probes were lyophilised using an Alpha 2-4 CD plus freeze-dryer (Christ, Germany) equipped with a high vacuum pump. 


\subsection{2) Reverse Phase Liquid Chromatography}

Analytical ultra-performance liquid chromatography (UPLC) was performed applying the Ultimate 3000 system (Thermo Scientific, USA) with the column Ace Excel (RP C-18, $100 \times 2.1 \mathrm{~mm}, 2 \mu \mathrm{m}, 100 \AA)$. Linear gradients of solvent $\mathrm{A}(0.1 \%$ aq. TFA) and solvent B1 (acetonitrile $+0.085 \%$ TFA) at $50{ }^{\circ} \mathrm{C}$ with a flow rate of $0.4 \mathrm{~mL} / \mathrm{min}$ were performed. Semi-preparative high-performance liquid chromatography (HPLC) was performed using a JASCO system (MD 2010 plus detector, DG-2080-53 degasser and PU-2080 plus pumps) with the semi-preparative columns Ace (RP C-18, $150 \times 10 \mathrm{~mm}$, $5 \mu \mathrm{m}, 300 \AA$ ) or Nucleodur ${ }^{\circledR}$ (RP C-18, $250 \times 10 \mathrm{~mm}, 5 \mu \mathrm{m}, 100 \AA$ ). Linear gradients of solvent $A$ and solvent $B 1$ or $B 2$ (methanol $+0.1 \%$ TFA) at RT with a flow rate of $6.5 \mathrm{~mL} / \mathrm{min}$ (Ace column) or $3 \mathrm{~mL} / \mathrm{min}$ (Nucleodur column) were performed. Samples were dissolved in TFE or water/methanol $(6: 4, v / v)$ prior to injection. UV detection was conducted at $215 \mathrm{~nm}, 225 \mathrm{~nm}, 254 \mathrm{~nm}$ and $280 \mathrm{~nm}$.

\subsection{3) Size Exclusion Chromatography}

For size exclusion chromatography $150 \mathrm{mg}$ of Sephadex G-50 Superfine column material (GE Healthcare, UK) were mixed with fusion buffer $(\sim 5 \mathrm{~mL})$. The mixture was transferred to a column $(0.5 \times 15 \mathrm{~cm})$ equipped with a porous polymer bed support at the bottom (Bio-Rad, USA). After the addition oflabelled liposomes, coloured fractions were collected.

\section{4) Solid Phase Peptide Synthesis}

\subsection{1) Loading of the First Amino Acid}

In the case of not preloaded amide resins (Nova PEG Rink amide or Rink amide $M B H A$ ), the first aa was loaded before starting subsequent SPPS cycles. The resin ( $0.13 \mathrm{mmol}$ of available amino groups) was swollen in $5 \mathrm{~mL} \mathrm{NMP}$ for $30 \mathrm{~min}$ and washed with NMP, DCM and NMP (each $3 \mathrm{x}$ ) using a BD Discardit ${ }^{T M}$ syringe $(10 \mathrm{~mL}$, acid/base resistant) with a polyethylene filter. The amino acid building block ( 5 eq.) and 
HOBt (5 eq.) were dissolved in $3.5 \mathrm{~mL}$ NMP. After the addition of $0.1 \mathrm{~mL}$ DIC (4.9 eq.), the solution was transferred to the resin. The aa was coupled for $600 \mathrm{~s}$ at $40{ }^{\circ} \mathrm{C}$ (assisted by the CEM Discover microwave reaction cavity, $20 \mathrm{~W}$ ). In the case of a positive Kaiser test (Section 5.4.2), the coupling reaction was repeated. If a low loading density was desired, coupling was performed only once and the remaining amino groups were acetylated (Section 5.4.6). Finally, the resin was washed using DMF, DCM and methanol $(5 \mathrm{x})$ and dried in vacuum for further synthesis.

\subsection{2) Kaiser Test}

To control the completion of the resin-loading (qualitative), the Kaiser test was performed: Stock solutions of ninhydrin in ethanol $(5 \mathrm{~g} / 100 \mathrm{~mL})$ and phenol in ethanol (80 g/20 mL) were prepared. Furthermore, $2 \mathrm{~mL}$ of a $1 \mathrm{mM}$ aq. potassium cyanide solution was mixed with $98 \mathrm{~mL}$ pyridine. Few beads of the resin were washed with ethanol, dried and added to a glass test tube. Then, $50 \mu \mathrm{L}$ of each stock solution were mixed and the mixture was added to the resin beads. The formulation was heated on a hotplate $\left(120^{\circ} \mathrm{C}\right)$ for 4-6 $\mathrm{min}$. When free amino groups on the resin were available, the colourless formulation turned to blue/purple (positive Kaiser test). When the test was negative, i.e. all amino groups were occupied by aa building blocks, the formulation turned to yellow (negative Kaiser test). The Kaiser test is useful to determine whether the resin-loading must be repeated or not. Resin-loading can only be controlled by the Kaiser test when amide yielding resins were used.

\subsection{3) Estimation of the Loading Density}

For determination of the loading density (LD), 5-10 $\mathrm{mg}$ of the resin was transferred to a graduated flask $(10 \mathrm{~mL})$. Then, $2 \mathrm{~mL}$ of DMF and $40 \mu \mathrm{L}$ of DBU were added. As reference the same sample without resin was prepared. The samples were gently agitated for $1 \mathrm{~h}$ and afterwards, they were filled to $10 \mathrm{~mL}$ with acetonitrile. For UV measurements, probes were diluted by a factor of $10(50 \mu \mathrm{L}$ sample in $450 \mu \mathrm{L}$ acetonitrile) and were transferred to a quartz cuvette $(1 \mathrm{~cm} \times 1 \mathrm{~cm})$. The absorption of the probes 
was detected at $304 \mathrm{~nm}$ (absorption maximum of dibenzofulvene) using the Jasco V-550 UV/vis-spectrophotometer ( V-500W Spectra Manager software, Fixed Wavelength Measurement mode). Three measurements (each 5 cycles) of the sample and the reference were recorded, respectively. The LD was calculated using following equation based on the Lambert-Beer's law:

$$
\mathrm{LD}=\frac{\left(A-A_{0}\right) \times 0.01 \mathrm{~L}}{\varepsilon \times d \times m}
$$

Thereby, LD is the loading density in $\mathrm{mmol} / \mathrm{g}, A$ is the absorption of the probe, $A_{0}$ is the absorption of the reference, $\varepsilon$ is the extinction coefficient of dibenzofulvene at $304 \mathrm{~nm}\left(7624 \mathrm{M}^{-1} \mathrm{~cm}^{-1}\right), d$ is the length of the cuvette in $\mathrm{cm}$ and $m$ is the mass of the used resin in grams. ${ }^{[189]}$

\subsection{4) Synthesis of PNA-LR/TMD Peptide Hybrids}

TMDs for the PNA based model peptides were automatically synthesised via Fmoc-solid phase peptide synthesis (Fmoc-SPPS) on a pre-loaded Wang resin ( $0.1 \mathrm{mmol}, 0.29-0.38 \mathrm{mmol} / \mathrm{g})$. In the case of $C$-terminal amide modified compounds, a manually pre-loaded Rink amide MBHA $(0.36 \mathrm{mmol} / \mathrm{g})$ or Nova PEG Rink amide resin $(0.18 \mathrm{mmol} / \mathrm{g})$ was used (Section 5.4.1). Syntheses of all TMDs were performed at $0.1 \mathrm{mmol}$ scales. Fmoc-SPPS was performed by using a Liberty peptide synthesiser equipped with a Discover microwave reaction cavity (CEM, USA): After swelling of the resin (60 min in NMP or DMF), the Fmoc deprotection reaction was conducted by a mixture of piperidine $(20 \%, v / v)$ and $\mathrm{HOBt}(0.1 \mathrm{M})$ in NMP $\left(\mathrm{RT} \rightarrow 75^{\circ} \mathrm{C}\right.$ for $210 \mathrm{~s}$, $35 \mathrm{~W}$ ). Coupling of the aa building blocks was achieved with $0.5 \mathrm{M} \mathrm{HBTU} / \mathrm{HOBt}$ ( $V_{\text {add }}=1 \mathrm{~mL}, 5$ eq.) and $2 \mathrm{M}$ DIPEA ( $V_{\text {add }}=0.5 \mathrm{~mL}, 10$ eq.) stock solutions in NMP (RT $\rightarrow 75^{\circ} \mathrm{C}$ for $300 \mathrm{~s}, 23 \mathrm{~W}$ ). The acetylation (capping) of unreacted amino groups was performed by a mixture of acetic acid anhydride $(2.3 \%, v / v)$, DIPEA $(4.7 \%, v / v)$ and HOBt (15 mM) in NMP (RT $\rightarrow 65^{\circ} \mathrm{C}$ for $60 \mathrm{~s}, 40 \mathrm{~W}$ ). The following aa building blocks were used for the preparation of stock solutions in NMP $\left(0.2 \mathrm{M}, V_{\text {add }}=2.5 \mathrm{~mL}\right.$, 5 eq.): 
- Fmoc-Ala-OH

- Fmoc-Arg(Pbf)-OH

- Fmoc-Asn(Trt)-OH

- Fmoc-Cys(Trt)-OH

- Fmoc-Gln(Trt)-OH

- Fmoc-Phe-OH

- Fmoc-Ser('Bu)-OH

- Fmoc-Thr( $\left.{ }^{\mathrm{B}} \mathrm{Bu}\right)-\mathrm{OH}$
- Fmoc-Gly-OH

- Fmoc-lle-OH

- Fmoc-Leu-OH

- Fmoc-Lys(Boc)-OH

- Fmoc-Met-OH

- Fmoc-Trp(Boc)-OH

- Fmoc-Tyr( ${ }^{(B u}$ Bu)-OH

- Fmoc-Val-OH.

All aas were introduced by double couplings. Special care was taken for the incorporation of cysteine and arginine residues. For Fmoc-Cys(Trt)-OH, the temperature of the microwave assisted coupling, capping and deprotection steps was reduced to $50{ }^{\circ} \mathrm{C}$ to avoid racemisation. Therefore, reaction time for the coupling reaction was elongated to $360 \mathrm{~s}$ ( 1 . RT for $120 \mathrm{~s} / 2$. RT $\rightarrow 50{ }^{\circ} \mathrm{C}$ for $240 \mathrm{~s}, 25 \mathrm{~W}$ ). For arginine, the reaction time of the coupling step was elongated to $30 \mathrm{~min}$ (1. RT for $25 \mathrm{~min} / 2$. RT $\rightarrow 75^{\circ} \mathrm{C}$ for $5 \mathrm{~min}, 25 \mathrm{~W}$ ). Capping and deprotection steps were performed as described above. The Fmoc protecting group of the $\mathrm{N}$-terminal aa was not cleaved. After synthesis, the resin was filtered off and washed successively with NMP and DCM (each $10 \mathrm{x}$ ). Then, the resin was dried in vacuum and a test cleavage was performed.

For subsequent manual elongation of the peptide chain with PNA-monomers, $5 \mu \mathrm{mol}$ of the resin bound peptide was transferred into a $B D$ Discardit ${ }^{T M}$ syringe $(2 \mathrm{~mL}$, acid/base resistant) equipped with a polyethylene filter. Initially, the resin was washed successively with NMP, DCM, methanol and NMP (each $5 \mathrm{x}$ ), swollen in NMP for 60 min and washed again with NMP, DCM and NMP (each $5 \mathrm{x}$ ). The following PNA monomers were used for decamer formation:

- Fmoc-adenine(Bhoc)-aeg-OH

- Fmoc-cytosine(Bhoc)-aeg-OH

- Fmoc-guanine(Bhoc)-aeg-OH

- Fmoc-thymine-aeg-OH.

The coupling cycle was started with Fmoc deprotection using piperidine in NMP (20 \%, $v / v, R T$ for $2 \times 5 \mathrm{~min}$ ), followed by washing with NMP, DCM and again NMP (each $4 \mathrm{x}$ ). For each coupling, $27.3 \mu \mathrm{mol}$ (5.45 eq.) PNA monomers were used. The PNA building 
blocks were dissolved in stock solutions of the coupling reagents HATU $(0.5 \mathrm{M}$, 5.30 eq.) and HOAt (2.0 M, 5.45 eq.) in NMP 5 min prior to the addition of the activator bases DIPEA (2.0 M, 5.45 eq.) and 2,6-lutidine (2.0 M, 8.20 eq.). Directly after the addition of the activatior bases the reaction mixture was transferred to the resin. The initial concentrations of PNA building blocks and HOAt in the reaction mixture were adjusted to $0.27 \mathrm{M}$, respectively. The concentration of HATU was adjusted to $0.26 \mathrm{M}$. DIPEA and 2,6-lutidine had concentrations of $0.27 \mathrm{M}$ and $0.41 \mathrm{M}$, respectively. The coupling reactions were performed at RT for $60 \mathrm{~min}$. All PNA building blocks were coupled twice. After successive washing with NMP, DCM and again NMP (each $3 \mathrm{x}$ ), capping with a mixture of acetic acid anhydride $(10 \%, v / v)$ and 2,6-lutidine $(20 \%, v / v)$ in NMP was performed (RT for $2 \times 5$ min). A final washing step with DIPEA in NMP (5\%, v/v), DCM and NMP (each $3 \mathrm{x}$ ) completed the SPPS cycle. After completing the PNA synthesis, the resin was thoroughly washed with NMP and DCM (each $10 \mathrm{x}$ ) and dried in vacuum.

\subsection{5) Synthesis of E3/K3-LR/TMD Peptide Hybrids}

E3/K3-LR/TMD peptide hybrids were synthesised via automated SPPS using a Liberty Blue peptide synthesiser equipped with a Discover microwave reaction cavity (CEM, USA). The same resins and aa building blocks as for the synthesis of TMDs of the PNA based model peptides were used. The synthesis scale was $0.05 \mathrm{mmol}$ and no capping was performed. After swelling of the resin in DMF for $60 \mathrm{~min}$, single couplings were applied for the first 10 aa building blocks. Subsequently, building blocks were coupled twice. For deprotection, a solution of piperidine (20\%, v/v) and Oxyma (0.1 M) in DMF was used. Microwave assisted deprotection was performed in two steps $\left(1 . \mathrm{RT} \rightarrow 75^{\circ} \mathrm{C}\right.$ for $15 \mathrm{~s}, 155 \mathrm{~W} / 2.90^{\circ} \mathrm{C}$ for $50 \mathrm{~s}, 30 \mathrm{~W}$ ). Stock solutions of $0.2 \mathrm{M}$ aa building blocks ( $V_{\text {add }}=1.25 \mathrm{~mL}, 5$ eq.), $0.50 \mathrm{M}$ Oxyma ( $V_{\text {add }}=0.5 \mathrm{~mL}, 5$ eq.) and $0.25 \mathrm{M}$ DIC ( $V_{\text {add }}=1 \mathrm{~mL}, 5$ eq.) were applied coupling. The coupling steps were conducted in two steps $\left(1\right.$. RT $\rightarrow 75^{\circ} \mathrm{C}$ for $15 \mathrm{~s}, 170 \mathrm{~W} / 2.90^{\circ} \mathrm{C}$ for $\left.110 \mathrm{~s}, 30 \mathrm{~W}\right)$. Cysteine and arginine were coupled under adjusted conditions to avoid racemization and $\delta$-lactam formation (cysteine: 1 . RT for $120 \mathrm{~s} / 2$. RT $\rightarrow 50{ }^{\circ} \mathrm{C}$ for $240 \mathrm{~s}, 35 \mathrm{~W}$; arginine: 1. RT for $1500 \mathrm{~s} / 2$. RT $\rightarrow 75^{\circ} \mathrm{C}$ for $\left.120 \mathrm{~s}, 30 \mathrm{~W}\right) .{ }^{[159]}$ After synthesis, the resin was 
filtered off and washed successively with DMF and DCM (each $10 \mathrm{x}$ ). Finally, the resin was dried in vacuum and a test cleavage was performed.

\subsection{6) Acetylation of K3 and iE3 Peptides}

$10 \mathrm{mg}$ of the resin with the corresponding peptide were transferred to a BD Discardit ${ }^{T M}$ syringe ( $2 \mathrm{~mL}$, acid/base resistant) equipped with a polyethylene filter. The resin was swollen in DMF for $30 \mathrm{~min}$ and subsequently, $1.5 \mathrm{~mL}$ of a solution of acetic acid anhydride $(10 \%, v / v)$ and 2,6-lutidine $(20 \%, v / v)$ in DMF were added to the resin. Acetylation was carried out twice under gentle agitation at RT for $5 \mathrm{~min}$. Afterwards, the resin was washed in DMF and DCM (each $3 \mathrm{x}$ ) and dried in vacuum.

\subsection{7) NBD Labelling of K3 Peptides}

$N$-terminal labelling of the $\mathrm{K} 3$ peptides with NBD was conducted at a $1 \mu \mathrm{mol}$ scale: $5 \mu \mathrm{mol}$ NBD-F ( $0.91 \mathrm{mg}, 5$ eq.) was dissolved in $95 \mu \mathrm{L}$ NMP and $5 \mu \mathrm{L}$ DIPEA (29 eq.) were added. The solution was directly transferred to the resin and the reaction was performed under light exclusion overnight. The resin was washed in DMF and DCM (each $5 \mathrm{x}$ ) and dried in vacuum.

\subsection{8) Cleavage from the Solid Support}

For the cleavage of the synthesised peptides from the solid support (resin) TFA doped with various scavengers was used. Small amounts of the resin (10-30 mg) were transferred to a $B D$ Discardit ${ }^{T M}$ syringe ( $2 \mathrm{~mL}$, acid/base resistant). Subsequently, for peptide cleavage appropriate solutions were added (1-2 mL):

- TMD:

TFA/EDT/water/TIS (87.5:5:5:2.5, $v / v / v / v)$

- aeg-PNA:

TFA/m-cresol/TIS (92.5:5:2.5, v/v/v)

- E3, K3:

TFA/water/TIS (95:2.5:2.5, $v / v / v)$

- PNA-LR/TMD:

TFA/EDT/m-cresol/TIS (87.5:5:5:2.5, $v / v / v / v)$ 
- E3/K3-LR/TMD: TFA/EDT/water/TIS (94:2.5:2.5:1, $v / v / v / v)$

- Cys-E3/SybT: TFA/aq. TCEP (10 mM)/water/TIS (88:5:5:2, $v / v / v / v) .{ }^{[190]}$

Cleavage from the resin was carried out at RT under gentle agitation for $2 \mathrm{~h}$. Then, the cleavage mixture was filtered and the solution was concentrated by using a nitrogen stream. The peptides were precipitated as white solids by the addition of cold $\left(-20^{\circ} \mathrm{C}\right)$ diethylether. The resulting suspension was centrifugated and the supernatant was discarded. The solid was washed with diethylether $(4 \mathrm{x})$ and dried under air. Finally, the crude products were dissolved in TFE/water $(1: 3, v / v)$ and lyophilised. All compounds were used without further purification for membrane fusion assays.

\section{5) Preparation of Model Membranes}

\subsection{1) Large Unilamellar Vesicles}

Preparation of stock solutions:

Stock solutions of the unlabelled lipids 1,2-dioleoyl-sn-glycero-3-phosphatidylcholine (DOPC), 1,2-dioleoyl-sn-glycero-3-phosphoethanolamine (DOPE) and cholesterol were prepared in chloroform $(20 \mathrm{mg} / \mathrm{mL}$, respectively). The same solvent was used for the labelled lipids NBD-DOPE and Rh-DOPE ( $2 \mathrm{mg} / \mathrm{mL}$, respectively). For model peptide stock solutions TFE was used and concentrations were determined photometrically (Section 5.6.6).

\section{Preparation of peptide/lipid films:}

Lipid and peptide stock solutions were added to a pre-mixed solution of chloroform and TFE yielding a chloroform/TFE ratio of 1:1 $(v / v)$ and a peptide/lipid ratio of 1:200 $(n / n)$. The lipid composition for the preparation of unlabelled (proteo-)liposomes was DOPC/DOPE/cholesterol $(50: 25: 25, n / n / n)$ and the total amount of lipids was $n_{\text {lipids }}=2.5 \mu \mathrm{mol}\left(n_{\text {peptides }}=12.5 \mathrm{nmol}\right)$. The films for the labelled proteoliposomes were composed of DOPC, DOPE, cholesterol, NBD-DOPE and Rh-DOPE (50:22:25:1.5:1.5, $n / n / n / n / n)$ and the corresponding model peptides. The removal of solvents was performed above the main transition temperatures $\left(T_{\mathrm{m}}\right)$ of the used lipids $\left(50{ }^{\circ} \mathrm{C}\right)$ by applying a nitrogen stream. The resulting thin peptide/lipid films at the test tube walls 
were incubated under reduced pressure at $50{ }^{\circ} \mathrm{C}$ for $12 \mathrm{~h}$ to remove residual organic solvent.

\section{Preparation of LUVs via extrusion:}

Organic solvent free peptide/lipid films were hydrated by adding $500 \mu \mathrm{L}$ of the fusion buffer (20 mM HEPES, 100 mM KCl, 1 mM EDTA, 1 mM DTT, pH = 7.4) and 3-6 glass beads (Clipids $=5 \mathrm{mM}$, Cpeptides $=25 \mu \mathrm{M}$ ). The samples were incubated under gentle agitation at $50{ }^{\circ} \mathrm{C}$ for $1-2 \mathrm{~h}$. Hydrated films were treated in a sonication bath for $15-30 \mathrm{~s}$ and agitated mechanically (vortexer for $5 \mathrm{~s}$ ) to remove remaining films from the test tube walls. Hydration of the films resulted in turbid suspensions which were extruded at least 21 times through a polycarbonate membrane (100 nm nominal pore diameter) by using a LiposoFast mini-extruder (Avestin, Canada) to produce almost clear (proteo-)liposome suspensions. ${ }^{[93]}$

\subsection{2) Preparation of Pore Spanning Membranes and Pro- teoliposomes of Different Sizes}

Porous (pore diameter of $1.2 \mu \mathrm{m}$ ) silicon nitride slides were coated by thin layers of titanium and gold. The gold surface was functionalised by using a solution of 6-mercapto-1-hexanol in $n$-propanol $(0.1 \mathrm{mM})$. GUVs with a lipid composition of POPE/POPS/DOPC/cholesterol/Atto488-DPPE (19:10:50:20:1, $n / n / n / n / n)$ and a peptide/lipid ratio of 1:250 were prepared by detergent removal and subsequent electroformation on indium tin oxide slides applying a sinusoidal voltage (1.6 $\mathrm{V}_{\mathrm{p}-\mathrm{p}}, 12 \mathrm{~Hz}$ for $3 \mathrm{~h}$ ). The obtained GUV suspension was directly added onto the porous slides and was incubated at RT for $30 \mathrm{~min}$. The bilayer formation was controlled fluorometrically using a confocal laser microscope. LUVs for the membrane fusion experiments on PSMs were also prepared by detergent removal from a micellar peptide/lipid/detergent mixture by using size exclusion chromatography and subsequent extrusion with polycarbonate membranes with nominal pore diameters of $400 \mathrm{~nm}$. The lipid composition and peptide/lipid ratio were equal to GUVs but as labelled lipids 1 mol-\% TxR-DHPE was used. ${ }^{[88]}$ SUVs for accompanying leaflet mixing assays were obtained through deter- 
gent depletion of the peptide/lipid/detergent mixture by using size exclusion chromatography. ${ }^{[9,191,192]}$ One proteoliposome sample was prepared with Atto488-DPPE and the other sample with Texas Red-DHPE (1 mol-\%, respectively).

\subsection{3) Preparation of Solid Supported Membranes and Col- loidal Probe Supported Membranes}

LUVs with the lipid composition of DOPC/DOPE/cholesterol/NBD-DOPE (50:24:25:1, $n / n / n / n)$ and a peptide/lipid ratio of 1:200 were prepared according to the protocol described in Section 5.5.1. The obtained LUV suspension was added to a glass-bottom (borosilicate) dish and was incubated at RT for $2 \mathrm{~h}$. Afterwards, the excess of liposomes was rinsed by HEPES buffer (20 mM HEPES, 100 mM KCl, 1 mM EDTA, 1 mM DTT, $\mathrm{pH}=7.4$ ). The coverage of the solid support was controlled with confocal laser microscopy and AFM imaging. For the preparation of the membrane coated colloidal probes, a glass (borosilicate) microsphere (diameter of $15 \mu \mathrm{m}$ ) was attached to a tipless cantilever using a micromanipulator and epoxy resin. Afterwards, the cantilever was cleaned in an argon plasma for $30 \mathrm{~s}$. Direct before starting the measurements, the colloidal probe was incubated in the LUV suspension (80-100 $\mu \mathrm{L}, \sim 5 \mathrm{mM}$ in HEPES buffer) in a hanging droplet at RT for $15 \mathrm{~min}$. The excess of proteoliposomes was removed by rinsing with HEPES buffer. ${ }^{[180]}$

\section{6) Modification of AFM tips and Peptide Attachment}

Commercially available AFM tips were coated by a 30-50 nm thick gold layer applying vapor disposition. When the cantilever approached the solid supported membrane doped with Cys-E3/SybT (peptide/lipid of 1:200) covalent bonds between the gold surface of the tip and the thiol groups of $\mathrm{N}$-terminal cysteines were formed (thioate bonds). Bond formation was detected through force events in force-distance curves. ${ }^{[184,185]}$ 


\section{7) Analytical Methods}

\subsection{1) Mass spectrometry}

Electrospray ionisation mass spectra (ESI-MS) were obtained by using the maXis mass spectrometer (Bruker, USA) with a time of flight (TOF) analyser. High resolution mass spectra (HR-MS) with a measuring accuracy of $<5 \mathrm{ppm}$ were received. Cations were detected in the range of 300-2900 m/z. Data analysis was realised by the Bruker Compass DataAnalysis 4.0 software.

\subsection{2) Dynamic Light Scattering}

Size determination of (proteo-)liposomes by dynamic light scattering (DLS) was performed using the DynaPro Titan (Wyatt) or the Zetasizer Nano S (Malvern) device. The (proteo-)liposome concentration was equal to the applied concentration during the leaflet mixing assays ( $185 \mu \mathrm{M}$ with regard to lipids before extrusion, lipid loss through extrusion was $15-50 \%$ ): The DynaPro Titan operated at $90^{\circ}$ concerning the detection of scattered laser light (right angle detection). Measurements were performed at $25^{\circ} \mathrm{C}$, the laser power was adjusted to 3-4 \% and 50 measurements per sample were recorded. Data analysis was conducted with the Dynamics 6.9.1 software. The Zetasizer Nano $S$ operated at $173^{\circ}$ concerning the detection angle (back angle detection). Measurements were carried out at $25^{\circ} \mathrm{C}$, the laser power was automatically adjusted through an attenuator and three measurements per sample were performed (correlation delay time of $63 \mathrm{~s}$ ). The control of the device and data capture were conducted by the Zetasizer Nano software.

\subsection{3) Nano Tracking Analysis}

Another technique applied for the determination of liposome sizes was the Nano tracking analysis (NTA). An optical microscope equipped with a LM14 measuring chamber and a LM10 HS CMOS camera (NanoSight Ltd., UK) was used. For the measurements, the (proteo-)liposome suspensions were diluted to a lipid concentration of $1 \mu \mathrm{M}$ 
(loss of lipids during extrusion not considered). Three samples of the examined (proteo-)liposome suspensions were measured. Camera sensitivity was adjusted to 13-14. Videos of $30 \mathrm{~s}$ were captured at RT. Thereby, more than 200 (proteo-)liposomes were tracked and analysed by the NTA 2.3 Analytical software.

\subsection{4) Phosphate Determination}

$10 \mu \mathrm{L}$ of (proteo)-liposome suspensions (10 $\mu \mathrm{L}$ water as reference) were diluted with $190 \mu \mathrm{L}$ water. $200 \mu \mathrm{L}$ of perchloric acid (70\% aq. solution) were added and lipids were digested at $220^{\circ} \mathrm{C}$ for $1 \mathrm{~h}$ (usage of a metal block on a hotplate). For the calibration curves, mixtures of water and an aq. solution of sodium dihydrogenphosphate monohydrate $(89 \mathrm{mg} / \mathrm{L})$ were prepared following Table 5.7.4-1. Each sample was prepared twice. $700 \mu \mathrm{L}$ of the freshly prepared reagents $\mathrm{A}$ (aq. solution of $0.45 \%(w / v)$ ammonium molybdate and $12.6 \%(w / v)$ perchloric acid) and reagent $B$ (aq. solution of $1.7 \%$ $(w / v)$ sodium ascorbate) were transferred to the digested samples and to the mixtures for the calibration curves, respectively. All samples were thoroughly mixed and heated at $80{ }^{\circ} \mathrm{C}$ for $7 \mathrm{~min}$ by using a water bath. ${ }^{[193]}$

Table 5.7.4-1 Mixtures of water and phosphate solution for calibration curves.

\begin{tabular}{|c|c|c|}
\hline $\boldsymbol{V}\left(\mathrm{H}_{2} \mathrm{O}\right)[\mu \mathrm{L}]$ & $\boldsymbol{V}\left(\mathrm{NaH}_{2} \mathrm{PO}_{4} \cdot \mathrm{H}_{2} \mathrm{O}\right)[\mu \mathrm{L}]$ & $\boldsymbol{m}(\mathrm{P})[\mu \mathrm{g}]$ \\
\hline 100.0 & 0.0 & 0.00 \\
62.5 & 37.5 & 0.75 \\
50.0 & 50.0 & 1.00 \\
37.5 & 62.5 & 1.25 \\
25.0 & 75.0 & 1.50 \\
0.0 & 100.0 & 2.00 \\
\hline
\end{tabular}

After cooling down, $300 \mu \mathrm{L}$ of the samples were transferred to $U V$-Star ${ }^{\circledR} 96$ well microplates (Greiner Bio-One, Germany) and the absorption of phosphomolybdate complexes was determined at $820 \mathrm{~nm}$ using the CLARIOstar microplate reader (BMG Labtech, Germany). For the calibration curves, detected absorption was plotted against the assumed mass of phosphorus. The obtained linear function was used to determine 
the mass of phosphorus of the (proteo-)liposome samples. The control of the device and data capture were conducted by the CLARIOstar software.

\subsection{5) SDS-PAGE}

For sodium dodecyl sulfate-polyacrylamide gel electrophoresis (SDS-PAGE) hand casted mini gels (thickness of $0.75 \mathrm{~mm}$ ) were used. Mixtures for resolving and stacking gels were prepared according to Table 5.7.5-1.

Table 5.7.5-1 Compositions of resolving and stacking gels. Given amounts are sufficient for two gels.

\begin{tabular}{|c|c|c|c|}
\hline component & composition & resolving gel & stacking gel \\
\hline Ethylene glycol & pure ( $\geq 99 \%)$ & $2.4 \mathrm{~mL}$ & - \\
\hline Gel buffer & $3 \mathrm{M}$ Tris- $\mathrm{HCl}+0,4 \%$ SDS $(w / v)$ & $2.0 \mathrm{~mL}$ & $1.0 \mathrm{~mL}$ \\
\hline acryl/bisacryl & aq. $40 \%(w / v)$ & $3.6 \mathrm{~mL}(19: 1, w / w)^{\mathrm{a}}$ & $0.5 \mathrm{~mL}(29: 1, w / w)^{\mathrm{b}}$ \\
\hline water & - & - & $2.5 \mathrm{~mL}$ \\
\hline$V_{\text {total }}$ & - & $8 \mathrm{~mL}$ & $4 \mathrm{~mL}$ \\
\hline APS & $40 \%$ in water $(w / v)$ & $8 \mu \mathrm{L}$ & $4 \mu \mathrm{L}$ \\
\hline TEMED & pure ( $\geq 99 \%)$ & $12 \mu \mathrm{L}$ & $16 \mu \mathrm{L}$ \\
\hline
\end{tabular}

Remarks: a, b) Ratio of acrylamide and methylenebisacrylamide of the aq. $40 \%$ stock solutions.

The polymerisation of acrylamides was started by APS and TEMED just before transferring the mixtures into the gel chambers. For stacking gels, $\sim 1.5 \mathrm{~cm}$ at the top of the chambers were kept free. After the addition of the stacking gel, a comb for the gel pockets was fixed. When polymerisation was completed, gels were stored at $4{ }^{\circ} \mathrm{C}$ in paper towels which were soaked by the running buffer. For SDS-PAGE runs, gels were fixed in a Mini PROTEAN Tetra Cell electrophoresis chamber (Bio-Rad, USA) which was filled with the Novex ${ }^{T M}$ tricine SDS running buffer (ThermoFisher Scientific, USA). As marker for the identification of peptides the Color Marker Ultra-low Range (1060-26600 Da, Sigma-Aldrich, USA) was used. $20 \mu \mathrm{L}$ of the proteoliposome samples (after ultra-centrifugation, Section 5.7.8) were mixed with $5 \mu \mathrm{L}$ of the Novex ${ }^{T M}$ tricine SDS sample buffer (ThermoFisher Scientific, USA) and were filled into the gel pockets. The runs were performed at $U=200 \mathrm{~V}$. The peptide bands were stained by using a commercially available rapid silver staining kit (Sigma-Aldrich, USA). 


\subsection{6) UV-Spectroscopy}

The concentrations of the model peptide stock solutions were determined by using the $V$-650 UV/vis-spectrophotometer equipped with the ETCS-761 temperature controller (JASCO, Germany). Data were captured by the V-650 Spectra Manager software. All measurements were performed at $25^{\circ} \mathrm{C}$ and the sample cell was flushed with nitrogen. The absorption was detected for 300s and the last 100 measured points were averaged and used for the calculation of the concentrations. The molar extinction coefficients of the PNA-LR/TMD peptide hybrids were estimated by the summation of the molar extinction coefficients of aeg-PNA monomers (nucleobases), tryptophans, tyrosines and cysteins at $260 \mathrm{~nm} \cdot{ }^{[194]}$ For E3/K3 based model peptides, the estimation of the molar extinction coefficients was conducted considering solely aas at $280 \mathrm{~nm}$ (Table 5.7.6-1). ${ }^{[195]}$

Table 5.7.6-1 Molar extinction coefficients of the nucleobases and aas.

\begin{tabular}{|l|c|c|}
\hline \multicolumn{1}{|c|}{ nucleobases/aas } & $\boldsymbol{\varepsilon}_{260 \mathrm{~nm}}\left[\mathbf{M}^{-1} \mathbf{c m}^{-1}\right]$ & $\boldsymbol{\varepsilon}_{280} \mathbf{~ m}\left[\mathbf{M}^{-1} \mathbf{c m}^{-1}\right]$ \\
\hline cytosine & 6600 & - \\
guanine & 11700 & - \\
thymine & 8600 & - \\
\hline adenine & 13700 & - \\
\hline tryptophan & 3400 & 5700 \\
tyrosine & 500 & 1300 \\
cysteine & 400 & 120 \\
\hline
\end{tabular}

The summation of the molar extinction coefficients of the nucleobases and/or aas yielded following molar extinction coefficients for the different model peptide groups (Table 5.7.6-2).

Table 5.7.6-2 Molar extinction coefficients of the model peptides.

\begin{tabular}{|l|c|c|}
\hline \multicolumn{1}{|c|}{ model peptides } & $\boldsymbol{\varepsilon}_{260} \mathbf{n m}\left[\mathbf{M}^{-1} \mathbf{c m}^{-1}\right]$ & $\left.\boldsymbol{\varepsilon}_{280} \mathrm{~nm}^{[} \mathbf{M}^{-1} \mathbf{c m}^{-1}\right]$ \\
\hline PNA-SybX(a) & 111700 & - \\
\hline PNA-SxY(a) & 104800 & - \\
(i)E3-SybX(a) & - & 14100 \\
K3-SxY(a) & - & 12100 \\
\hline
\end{tabular}


The concentrations of peptide stock solutions were calculated from the Lambert-Beer's law:

$$
c=\frac{A}{\varepsilon \times d}
$$

Thereby, $c$ is the concentration of the peptides in $\mathrm{mol} / \mathrm{L}, A$ is the detected absorption at $260 \mathrm{~nm}$ or $280 \mathrm{~nm}, \varepsilon$ is the estimated molar extinction coefficient in $\mathrm{M}^{-1} \mathrm{~cm}^{-1}$ and $d$ is the length of the quartz-cuvette in $\mathrm{cm}$.

\subsection{7) Spectrofluorimetry}

Fluorescence spectra were obtained by applying the FP-6200 spectrofluorometer equipped with the ETC-272T temperature controller (JASCO, Germany). All measurements were conducted at $25^{\circ} \mathrm{C}$ and the sample cell was flushed with nitrogen. The samples were excited at $460 \mathrm{~nm}$. Emission spectra and time course measurements of emission were detected by using the parameters of Table 5.7.7-1. The control of the device and data capture were performed by the FP-6200 Spectra Manager software.

Table 5.7.7-1 Standard parameters for spectrofluorimetry.

\begin{tabular}{|l|c|c|}
\hline \multicolumn{1}{|c|}{ parameter } & spectrum measurement & time course measurement \\
\hline detection wavelength(s) & $500-610 \mathrm{~nm}$ & $535 \mathrm{~nm}$ \\
response & fast & $1 \mathrm{~s}$ \\
data pitch & $1 \mathrm{~nm}$ & $1 \mathrm{~s}$ \\
sensitivity & high & high \\
bandwidth & $5 \mathrm{~nm}(\mathrm{em}.) / 5 \mathrm{~nm}(\mathrm{ex})$. & $5 \mathrm{~nm}(\mathrm{em}.) / 5 \mathrm{~nm}(\mathrm{ex})$. \\
scanning speed & $125 \mathrm{~nm} / \mathrm{min}$ & - \\
measurement time & - & $1200 \mathrm{~s}$ \\
\hline
\end{tabular}

\subsubsection{1) Total Leaflet Mixing (TLM) Assay}

The FRET donor NBD-DOPE (emission maximum at $535 \mathrm{~nm}$ ) and the FRET acceptor Rh-DOPE (emission maximum at $585 \mathrm{~nm}$ ) were used as fluorescent probes. The FRET effect between NBD and Rh was exploited to detect leaflet mixing. $10 \mu \mathrm{L}$ of 
labelled and $40 \mu \mathrm{L}$ of unlabelled (proteo-)liposomes (preparation described in Section 5.5.1) were mixed in a quartz cuvette filled with $1300 \mu \mathrm{L}$ fusion buffer ( $20 \mathrm{mM}$ HEPES, $100 \mathrm{mM} \mathrm{KCl}, 1 \mathrm{mM}$ EDTA, $1 \mathrm{mM}$ DTT (pH = 7.4)) and equipped with a stirrer. Lipid concentrations of the (proteo-)liposome suspensions were estimated by standard phosphate determination assays (Section 5.7.4): the concentrations of the labelled proteoliposome samples were in the range of $3.0-4.0 \mathrm{mM}(20-40 \%$ lipid loss through extrusion) and the concentrations of the unlabelled samples were in the range of 2.5-4.3 mM (15-50\% lipid loss through extrusion). The total lipid concentration in the cuvette was estimated to be between 90 and $160 \mu \mathrm{M}$. The change of the donor emission intensity was plotted as:

$$
F_{\text {norm TLM }}=\frac{F_{t \mathrm{TLM}}-F_{\operatorname{min~TLM}}}{F_{\max \mathrm{TLM}}-F_{0 \mathrm{TLM}}}
$$

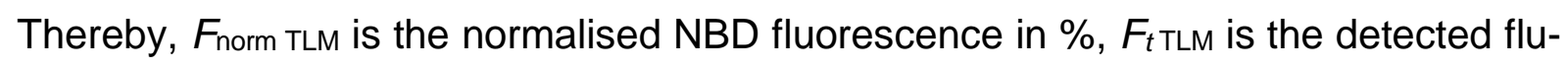
orescence after the addition of unlabelled (proteo-)liposomes at the time $t, F_{\min }$ TLM is the minimal detected fluorescence of the time course measurements, $F_{\max }$ TLM (from spectrum measurements) is the maximal fluorescence after the addition of $30 \mu \mathrm{L}$ detergent ( $10 \%$ aq. Triton X-100, v/v, Carl Roth) and $F_{0}$ TLM (from spectrum measurements) is the fluorescence of labelled proteoliposomes before the addition of unlabelled liposomes at $535 \mathrm{~nm}$. Leaflet mixing was detected for $1200 \mathrm{~s}$ and the detergent was added in several rounds until no further increase of NBD emission was detected ( $1^{\text {st }}$ round: $30 \mu \mathrm{L}, 2^{\text {nd: }}: 20 \mu \mathrm{L}, 3^{\text {rd: }}: 20 \mu \mathrm{L}$ ).

\subsubsection{2) Inner Leaflet Mixing (ILM) Assay}

$100 \mu \mathrm{L}$ of a suspension of labelled proteoliposomes (3.0-4.0 mM) were diluted with $200 \mu \mathrm{L}$ fusion buffer. NBD fluorescence at $535 \mathrm{~nm}$ was detected for $\sim 60 \mathrm{~s}$. Then, $100 \mu \mathrm{L}$ of a freshly prepared solution of sodium dithionite (DT) in fusion buffer (50 mM) were added. After decrease and subsequent stabilisation of the NBD emission, the excess of DT was removed by size exclusion chromatography (Section 5.3.3). The NBD reduction was carried out immediately before starting the ILM measurements. 
Standard normalisation of ILM measurements over time was conducted by using following equation:

$$
F_{\text {norm ILM }}=\frac{F_{t \text { ILM }}-F_{\text {min ILM }}}{F_{\max \text { TLM }}-F_{0 \text { ILM }}}
$$

Thereby, $F_{\text {norm ILM }}$ is the normalised NBD fluorescence in \%, $F_{\mathrm{t} \text { ILM }}$ is the detected fluorescence after the addition of unlabelled (proteo-)liposomes at the time $t, F_{\text {min ILM is the }}$ minimal detected fluorescence of the time course measurements, $F_{\text {max TLM (from spec- }}$ trum measurements) is the maximal fluorescence of DT-untreated labelled liposomes after the addition of $30 \mu \mathrm{L}$ detergent (10\% aq. Triton $X-100, v / v)$, i.e. fluorescence derives from both leaflets, and $F_{0}$ ILM (from spectrum measurements) is the fluorescence of DT-treated labelled proteoliposomes before unlabelled (proteo-)liposomes were added, i.e. fluorescence derives solely from inner leaflets. Leaflet mixing was detected for $1200 \mathrm{~s}$ and the detergent was added in several rounds until no further increase of NBD emission was detected ( $1^{\text {st }}$ round: $30 \mu \mathrm{L}, 2^{\text {nd }}: 20 \mu \mathrm{L}, 3^{\text {rd: }} 20 \mu \mathrm{L}$ ). An alternative mode for the normalisation of ILM measurements is given by the following equation:

$$
F_{\text {norm ILM }}=\frac{F_{t \text { ILM }}-F_{\min \text { ILM }}}{F_{\max \text { ILM }}-F_{0 \text { ILM }}}
$$

The detected fluorescence values are the same as described for equation 4 except for

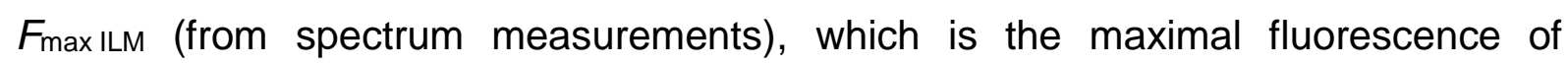
DT-treated labelled proteoliposomes after the addition of $30 \mu \mathrm{L}$ (or more) detergent, i.e. fluorescence derives solely from the inner leaflets. This mode of normalisation is useful to increase the resolution of ILM assays (Section 3.4.1). 


\subsubsection{3) Standard Deviation}

Both TLM and ILM assays were performed three times with different (preoteo-)liposome samples (same peptide probes). The standard deviation $S$ was calculated by:

$$
S=\sqrt{\frac{1}{n(n-1)} \times \sum_{t}\left(x_{\mathrm{t}}-\bar{x}\right)^{2}}
$$

Thereby, $n$ is the number of individual measurements, $x_{\mathrm{t}}$ are the measured data of individual mesurements at the time $t$ and $\bar{x}$ is the average of the measured data at the time $t$. Standard deviations were multiplied by the Student's factor $t=3.182(\mathrm{n}=3$, $95 \%$ certainty).

\subsubsection{4) Trigger of Membrane Fusion by Soluble Peptidic Components}

For the leaflet mixing assays concerning the examination of the membrane fusion triggering effect of soluble peptidic components, solely time course measurements (1600 s) were performed. $10 \mu \mathrm{L}$ of the labelled proteoliposomes were added to $1300 \mu \mathrm{L}$ fusion buffer and the fluorescence was detected for $\sim 120 \mathrm{~s}$. Subsequently, $40 \mu \mathrm{L}$ of unlabelled proteoliposomes were added. After stabilisation of the NBD fluorescence, soluble peptidic components $(0.5 \mathrm{mM})$ in different levels of excess $(4,24$ and 40 eq.) concerning the amount of membrane anchored model peptides in the cuvette ( $1.25 \mathrm{nmol}$, peptide loss through extrusion was not considered) were added. Approximately $1200 \mathrm{~s}$ after the addition of peptidic components, the detergent was added until no further increase of NBD fluorescence was observed (30 $\mu \mathrm{L}$ or more of $10 \%$ aq. Triton $X .-100, v / v)$ Measurements were normalised by:

$$
F_{\text {norm TLM }}=\frac{F_{t \text { TLM }}-F_{0 \text { TLM }}}{F_{\max T L M}-F_{0 \text { TLM }}}
$$

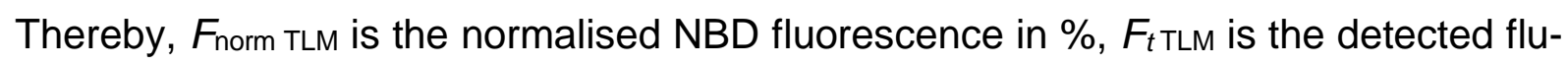
orescence after the addition of unlabelled proteoliposomes at the time $t, F_{0}$ TLM is the averaged fluorescence before the addition of unlabelled proteoliposomes, $F_{\max }$ TLM is the averaged fluorescence after the last addition of detergent. ILM assays were not performed. 


\subsubsection{5) Determination of Lipid Loss Through Detection of the Fluo- rescence of Rh-DOPE}

Another option for the estimation of the loss of lipid material beside the standard phosphate test (Section 5.7.4) is the detection of the Rh fluorescence at $585 \mathrm{~nm}$ (excitation at $560 \mathrm{~nm}$ ). For the estimation of lipid loss during extrusion, $2 \mu \mathrm{L}$ of the MLV suspension ( $5 \mathrm{mM}$ ) before extrusion and $2 \mu \mathrm{L}$ of the LUV suspension after extrusion were taken. For the estimation of the lipid loss during size exclusion chromatography, $10 \mu \mathrm{L}$ of a diluted LUV suspension (1.25 mM, lipid loss through extrusion not considered) before size exclusion chromatography and 10.0-12.5 $\mu \mathrm{L}$ (depending on the volume of the collected coloured LUV suspension) after size exclusion chromatography were used. For fluorescence measurements (spectrum measurements, 575-610 nm) samples were diluted with $1300 \mu \mathrm{L}$ fusion buffer. The detected intensity values of the fluorescence at $585 \mathrm{~nm}$ were directly used to estimate the loss of lipid material (Section 3.4.1).

\subsubsection{6) Proteoliposome Stability Test}

Labelled proteoliposome suspensions were treated with sodium dithionite (DT) and the fluorescence of NBD-DOPE was detected as described for ILM assays (Section 5.7.7.2). The stability of fluorescence signals before and after the addition of DT $(50 \mathrm{mM})$ was evaluated by the slopes of the fluorescence curves. The slope before the addition of DT (S before) was calculated by using the intensity values of fluorescence at $0 \mathrm{~s}$ and $30 \mathrm{~s}$ after starting the measurement $\left(F_{1}(0 \mathrm{~s})\right.$ and $\left.F_{2}(30 \mathrm{~s})\right)$. The slope after the addition of DT ( $S_{\text {after }}$ ) was calculated by using the intensity values at $120 \mathrm{~s}$ and $150 \mathrm{~s}$ after the addition of DT $\left(F_{1}(120 \mathrm{~s})\right.$ and $\left.F_{2}(150 \mathrm{~s})\right)$. Sbefore and $S_{\text {atter }}$ were calculated by equation 8:

$$
S_{\text {before } / \text { after }}=\frac{F_{2}-F_{1}}{30 \mathrm{~s}}
$$

When Sbefore was $>0.020$ a.u./s, proteoliposomes were evaluated as unstable. When Sbefore was $<0.020 \mathrm{a} . \mathrm{u} . / \mathrm{s}$, proteoliposomes were evaluated as stable. For $S_{\text {after }}<-0.040$ a.u./s, proteoliposomes were designated as unstable. For 
$S_{\text {after }}>-0.040$ a.u./s, proteoliposomes were designated as stable. Values given in Table 3.4.2-1 were averaged over three measurements.

\subsubsection{7) Peptide Insertion Test Based on Fluorescent Probes}

$100 \mu \mathrm{L}$ of proteoliposomes (Cpeptides $=25 \mu \mathrm{M}$ ), $100 \mu \mathrm{L}$ of liposomes or $100 \mu \mathrm{L}$ of the fusion buffer were mixed with $10 \mu \mathrm{L}$ of the NBD-K3 solution in fusion buffer $\left(C_{\mathrm{NBD}-\mathrm{K}}=250 \mu \mathrm{M}\right)$, respectively. All three mixtures were prepared twice and were gently agitated at $\mathrm{RT}$ for $1 \mathrm{~h}$. For the removal of unbound NBD-K3, one half of the mixtures was dialysed using the Slide-A-Lyzer MINI (Thermo Scientific, USA) dialysis chambers with a MWCO of $20 \mathrm{kDa}$. Samples were dialysed against $14 \mathrm{~mL}$ fusion buffer. After $3 \mathrm{~h}$ the buffer solution was exchanged and dialysis was resumed overnight. The other half of samples was not dialysed. The not dialysed mixtures of proteoliposomes/NBD-K3, liposomes/NBD-K3 and fusion buffer/NBD-K3 were used to determine calibration curves. For that, a serial dilution of seven samples with known concentrations (each diluted with fusion buffer by the factor of two) was prepared. The calibration for each mixture was necessary, because NBD fluorescence is highly influenced by the hydrophobicity of the medium. ${ }^{[173]}$ Moreover, the fusion buffer/NBD-K3 mixture served as control for the efficiency of dialysis concerning the fluorescent NBD-K3 peptides. The liposome/NBD-K3 mixture was used to evaluate the affinity of NBD-K3 to model membranes. The fluorescence at $535 \mathrm{~nm}$ was detected by spectrum measurements (500-600 nm) and was used to calculate the concentration of bound (not removed by dialysis) NBD-K3 with the help of the determined linear calibration curves.

\subsection{8) Peptide Insertion Test Based on Ultra-Centrifugation}

Centrifuge tubes were thoroughly cleaned with an aq. solution of $2 \%(v / v)$ Hellmanex III (Hellma, Germany). After rinsing with ultra-pure water, tubes were dried. $50 \mu \mathrm{L}$ of a solution of $80 \%$ Histodenz (Sigma-Aldrich, USA) in fusion buffer $(w / v)$ were mixed with $50 \mu \mathrm{L}$ of the proteoliposome suspensions (2.5-4.0 mM). For control experiments, both proteoliposomes with threefold amounts of peptides $(37.5 \mathrm{nmol}$, peptide/lipid ratio of 1:67 $(n / n)$ ) and liposomes were prepared (for preparation see Section 


\section{Experimental Part}

5.5.1). The liposomes were mixed with peptides dissolved in TFE (12.5 nmol). The individual mixtures were overlayed with $50 \mu \mathrm{L}$ of a $30 \%$ Histodenz solution $(w / v)$ and $30 \mu \mathrm{L}$ of fusion buffer. Thereby, it was important that layers were not mixed with each other. The samples were centrifuged at $4^{\circ} \mathrm{C}$ with $50000 \mathrm{rpm}(350000 \mathrm{~g})$ for $1.5 \mathrm{~h}$ in the Centrikon T-1065 centrifuge (Kontron Instruments, Germany) by using a TST60.4 rotor $\left(r_{\max }=125 \mathrm{~mm}\right)$. After centrifugation, samples were aliquoted $(20 \mu \mathrm{L})$ and prepared for SDS-PAGE (Section 5.6.5).

\section{8) Calculation of the Rate of Winding of $\alpha$-Helical Coiled Coils}

The distance for an entire turn of coiled-coil forming a-helices around each other is known as pitch $(P)$. $P$ depends on the radius of the coiled coil $\left(r_{0}\right)$, the number of aas per helical turn $(\alpha)$ and the axial rise per aa $(h)$ of the single $\alpha$-helices. The relationship between $P, r_{0}$ and $\alpha$ is given by:

$$
P=\frac{2 \pi}{\Delta t} \times \sqrt{h^{2}-\left(r_{0} \times \Delta t\right)^{2}}
$$

Thereby, $\Delta t$ is the twist differential and is calculated by equation 10 :

$$
\Delta t=2 \pi \times \frac{1}{\frac{1}{\alpha}-\frac{1}{3.5}}
$$

$\alpha$ is typically 3.6 and thus, $\Delta t$ is $-791.68 .^{[119]}$ Furthermore, $h$ for $\alpha$-helices is $0.15 \mathrm{~nm}$. The radii $r_{0}$ of the E3/K3 coiled coil and the Syb2/Sx1A coiled coil of the SNARE complex were determined by NMR solution and crystal structures by using the Pymol 0.99 rc6 software. ${ }^{[120,127]}$ Three distance (diameter) measurements between homogeneously distributed Ca-atoms of the peptide backbones were performed and averaged. The calculated values for the pitches of E3/K3 and Syb2/Sx1 A coiled coils were used to calculate the number of aas per pitch. Subsequently, $360^{\circ}$ were divided by these numbers of aas yielding the turning angle per aa which was used as a value for the rate of winding of the coiled coils. 


\section{9) Analytical Data}

\subsection{1) Transmembrane Domains}

Peptides without recognition motifs were synthesised as described in Section 5.4.5. Cleaved peptides were dissolved in methanol/TFE/formic acid $(10 / 10 / 1, v / v / v)$ for mass spectrometric measurements.

Syb2-TMD

H-KRKYWWKNLKMMIILGVICAIILIIIIVYFST-OH

$\left[\mathrm{C}_{190} \mathrm{H}_{308} \mathrm{~N}_{42} \mathrm{O}_{38} \mathrm{~S}_{3}, 3884.99\right]$

MS (ESI) $m / z: \quad 777.9[\mathrm{M}+5 \mathrm{H}]^{5+}, 972.1[\mathrm{M}+4 \mathrm{H}]^{4+}, 1295.8[\mathrm{M}+3 \mathrm{H}]^{3+}$, $1943.1[\mathrm{M}+2 \mathrm{H}]^{2+}$, deconvoluted $3884.3[\mathrm{M}]$.

HR-MS (ESI) $m / z$ : calcd. for $\mathrm{C}_{190} \mathrm{H}_{313} \mathrm{~N}_{42} \mathrm{O}_{38} \mathrm{~S}_{3}[\mathrm{M}+5 \mathrm{H}]^{5+} 777.8608$, found 777.8604;

calcd. for $\mathrm{C}_{190} \mathrm{H}_{312} \mathrm{~N}_{42} \mathrm{O}_{38} \mathrm{~S}_{3}[\mathrm{M}+4 \mathrm{H}]^{4+} 972.0742$, found 972.0750;

calcd. for $\mathrm{C}_{190} \mathrm{H}_{311} \mathrm{~N}_{42} \mathrm{O}_{38} \mathrm{~S}_{3}[\mathrm{M}+3 \mathrm{H}]^{3+} 1295.7631$, found 1295.7646 .

$\underline{\text { Sx1A-TMD }}$

H-KYQSKARRKKIMIIICCVILGIIIASTIGGIFG-OH

$\left[\mathrm{C}_{165} \mathrm{H}_{288} \mathrm{~N}_{44} \mathrm{O}_{39} \mathrm{~S}_{3}, 3608.57\right]$

MS (ESI) m/z: $722.6[\mathrm{M}+5 \mathrm{H}]^{5+}, 903.0[\mathrm{M}+4 \mathrm{H}]^{4+}, 1203.7[\mathrm{M}+3 \mathrm{H}]^{3+}$, deconvoluted 3608.1 [M].

HR-MS (ESI) $m / z$ : calcd. for $\mathrm{C}_{165} \mathrm{H}_{293} \mathrm{~N}_{44} \mathrm{O}_{39} \mathrm{~S}_{3}[\mathrm{M}+5 \mathrm{H}]^{5+} 722.6299$, found 722.6288;

calcd. for $\mathrm{C}_{165} \mathrm{H}_{292} \mathrm{~N}_{44} \mathrm{O}_{39} \mathrm{~S}_{3}[\mathrm{M}+4 \mathrm{H}]^{4+} 903.0355$, 


\section{Experimental Part}

found 903.0351;

calcd. for $\mathrm{C}_{165} \mathrm{H}_{291} \mathrm{~N}_{44} \mathrm{O}_{39} \mathrm{~S}_{3}[\mathrm{M}+3 \mathrm{H}]^{3+} 1203.7116$,

found 1203.7115 .

\subsection{2) Acetylated iE3 and K3 Peptides}

Peptides were synthesised according to the protocols described in Sections 5.4.5 and 5.4.6. Cleaved peptides were dissolved in methanol/formic acid $(10: 1, v / v)$ for mass spectrometric measurements.

$\underline{\text { Ac-iE3 }}$

\section{Ac-KELAAIEKELAAIEKELAAIE- $\mathrm{NH}_{2}$}

[ $\left.\mathrm{C}_{104} \mathrm{H}_{179} \mathrm{~N}_{25} \mathrm{O}_{34}, 2323.72\right]$

HPLC (Nucleodur column, RP-C18, $250 \mathrm{~mm} \times 10 \mathrm{~mm}, 5 \mu \mathrm{m}, 100 \AA$, gradient: $60-100 \%$ of solvent $B 2$ for $40 \mathrm{~min}$ ): $t_{\mathrm{R}}=18.2 \mathrm{~min}$.

MS (ESI) $\mathrm{m} / \mathrm{z}: \quad 581.8[\mathrm{M}+4 \mathrm{H}]^{4+}, 775.4[\mathrm{M}+3 \mathrm{H}]^{3+}, 1162.7[\mathrm{M}+2 \mathrm{H}]^{2+}$, deconvoluted $2323.3[\mathrm{M}]$.

HR-MS (ESI) $m / z$ : calcd. for $\mathrm{C}_{104} \mathrm{H}_{183} \mathrm{~N}_{25} \mathrm{O}_{34}[\mathrm{M}+4 \mathrm{H}]^{4+} 581.8342$, found 581.8329;

calcd. for $\mathrm{C}_{104} \mathrm{H}_{182} \mathrm{~N}_{25} \mathrm{O}_{34}[\mathrm{M}+3 \mathrm{H}]^{3+} 775.4431$, found 775.4441 ;

calcd. for $\mathrm{C}_{104} \mathrm{H}_{181} \mathrm{~N}_{25} \mathrm{O}_{34}[\mathrm{M}+2 \mathrm{H}]^{2+} 1162.6611$, found 1162.6603 . 


\section{Experimental Part}

$\underline{\mathrm{Ac}-\mathrm{K} 3}$

\section{Ac-KIAALKEKIAALKEKIAALKE-NH ${ }_{2}$}

$\left[\mathrm{C}_{107} \mathrm{H}_{194} \mathrm{~N}_{28} \mathrm{O}_{28}, 2320.90\right]$

HPLC (Nucleodur column, RP-C18, 250 mm x 10 mm, 5 m, $100 \AA$, gradient: 40-80\% of solvent B2 for $40 \mathrm{~min}$ ): $t_{R}=23.8 \mathrm{~min}$.

MS (ESI) $m / z: \quad 465.1[\mathrm{M}+5 \mathrm{H}]^{5+}, 581.1[\mathrm{M}+4 \mathrm{H}]^{4+}, 774.5[\mathrm{M}+3 \mathrm{H}]^{3+}$, $1161.2[\mathrm{M}+2 \mathrm{H}]^{2+}$, deconvoluted $2320.5[\mathrm{M}]$.

HR-MS (ESI) m/z: calcd. for $\mathrm{C}_{107} \mathrm{H}_{199} \mathrm{~N}_{28} \mathrm{O}_{28}[\mathrm{M}+5 \mathrm{H}]^{5+} 465.1002$, found 465.1000;

calcd. for $\mathrm{C}_{107} \mathrm{H}_{198} \mathrm{~N}_{28} \mathrm{O}_{28}[\mathrm{M}+4 \mathrm{H}]^{4+} 581.1234$, found 581.1236;

calcd. for $\mathrm{C}_{107} \mathrm{H}_{197} \mathrm{~N}_{28} \mathrm{O}_{28}[\mathrm{M}+3 \mathrm{H}]^{3+} 774.4955$, found 774.4958;

calcd. for $\mathrm{C}_{107} \mathrm{H}_{196} \mathrm{~N}_{28} \mathrm{O}_{28}[\mathrm{M}+2 \mathrm{H}]^{2+} 1161.2396$, found 1161.2390 .

\subsection{3) NBD Labelled K3 Peptide}

The peptide was synthesised as described in Sections 5.4.5 and 5.4.7. The cleaved peptide was dissolved in methanol/formic acid $(10: 1, v / v)$ for mass spectrometric measurements.

\section{$\underline{\text { NBD-K3 }}$}

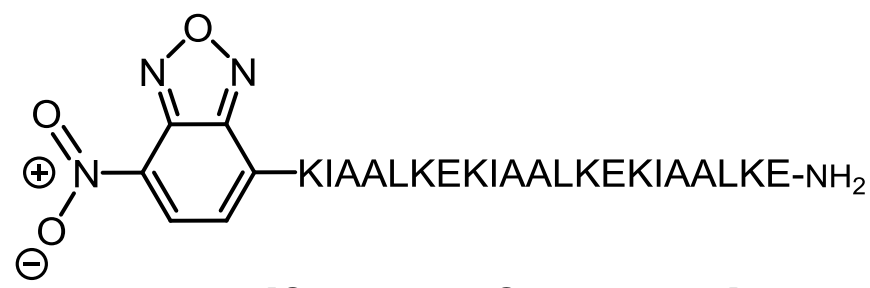

$\left[\mathrm{C}_{111} \mathrm{H}_{193} \mathrm{~N}_{31} \mathrm{O}_{30}, 2441.95\right]$

HPLC (Nucleodur column, RP-C18, 250 mm x 10 mm, 5 m, $100 \AA$, gradient: 40-80\% of solvent B2 for $40 \mathrm{~min}): t_{R}=25.0 \mathrm{~min}$. 
MS (ESI) $m / z: \quad 489.3[\mathrm{M}+5 \mathrm{H}]^{5+}, 611.4[\mathrm{M}+4 \mathrm{H}]^{4+}, 814.9[\mathrm{M}+3 \mathrm{H}]^{3+}$, $1221.8[\mathrm{M}+2 \mathrm{H}]^{2+}$, deconvoluted $2441.5[\mathrm{M}]$.

HR-MS (ESI) $m / z$ : calcd. for $\mathrm{C}_{111} \mathrm{H}_{198} \mathrm{~N}_{31} \mathrm{O}_{30}[\mathrm{M}+5 \mathrm{H}]^{5+} 489.2984$, found 489.2980;

calcd. for $\mathrm{C}_{111} \mathrm{H}_{197} \mathrm{~N}_{31} \mathrm{O}_{30}[\mathrm{M}+4 \mathrm{H}]^{4+} 611.3712$, found 611.3722;

calcd. for $\mathrm{C}_{111} \mathrm{H}_{196} \mathrm{~N}_{31} \mathrm{O}_{30}[\mathrm{M}+3 \mathrm{H}]^{3+} 814.8259$, found 814.8262;

calcd. for $\mathrm{C}_{111} \mathrm{H}_{195} \mathrm{~N}_{31} \mathrm{O}_{30}[\mathrm{M}+2 \mathrm{H}]^{2+} 1221.7352$, found 1221.7351.

\subsection{4) PNA-LR/TMD Peptide Hybrids}

Peptides were synthesised according to the protocol described in Section 5.4.4. Cleaved peptides were dissolved in methanol/TFE/formic acid $(4: 1: 1, v / v / v)$ for mass spectrometric analysis.

\subsubsection{1) PNA1/SybX(a) Peptides}

\section{PNA1/SybT}

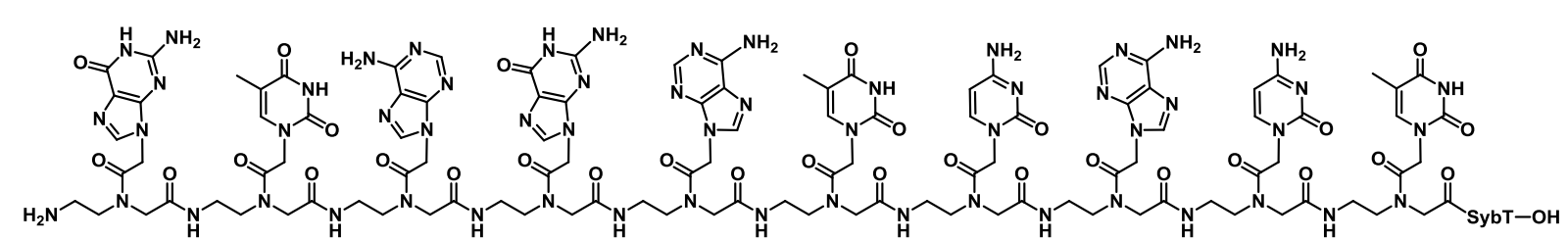

$\left[\mathrm{C}_{298} \mathrm{H}_{441} \mathrm{~N}_{99} \mathrm{O}_{68} \mathrm{~S}_{3}, 6594.61\right]$

UPLC (ACE Excel 2 column, RP-C18, $100 \mathrm{~mm} \times 2.1 \mathrm{~mm}, 2 \mu \mathrm{m}, 100 \AA$, gradient: $60-90 \%$ of solvent $\mathrm{B} 1$ for $15 \mathrm{~min}): t_{R}=11.0 \mathrm{~min}$.

MS (ESI) m/z: $\quad 943.1[\mathrm{M}+7 \mathrm{H}]^{7+}, 1099.9[\mathrm{M}+6 \mathrm{H}]^{6+}, 1319.9[\mathrm{M}+5 \mathrm{H}]^{5+}$, deconvoluted $6594.3[\mathrm{M}]$. 


\section{Experimental Part}

HR-MS (ESI) $m / z$ : calcd. for $\mathrm{C}_{298} \mathrm{H}_{447} \mathrm{~N}_{99} \mathrm{O}_{68} \mathrm{~S}_{3}[\mathrm{M}+6 \mathrm{H}]^{6+} 1100.0632$, found 1100.0649;

calcd. for $\mathrm{C}_{298} \mathrm{H}_{446} \mathrm{~N}_{99} \mathrm{O}_{68} \mathrm{~S}_{3}[\mathrm{M}+5 \mathrm{H}]^{5+} 1319.8744$, found 1319.8776 .

\section{PNA1/SybE}

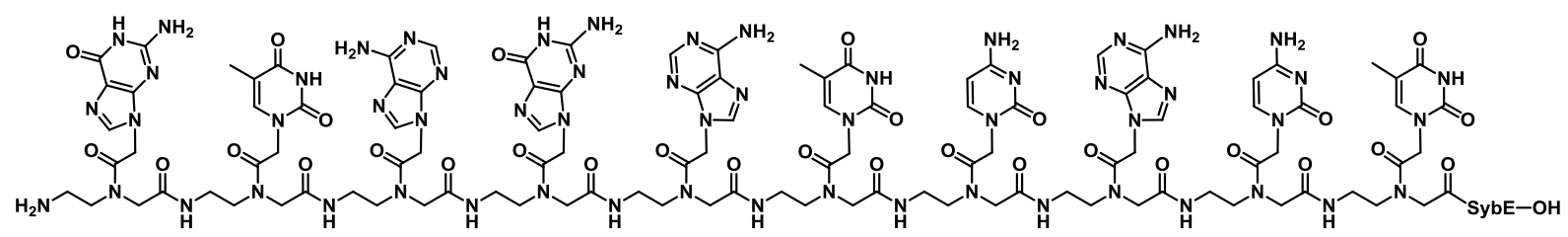

$\left[\mathrm{C}_{299} \mathrm{H}_{441} \mathrm{~N}_{99} \mathrm{O}_{69} \mathrm{~S}_{3}, 6622.62\right]$

UPLC (ACE Excel 2 column, RP-C18, $100 \mathrm{~mm} \times 2.1 \mathrm{~mm}, 2 \mu \mathrm{m}, 100 \AA$, gradient: $60-90 \%$ of solvent $\mathrm{B} 1$ for $15 \mathrm{~min}): t_{R}=9.2 \mathrm{~min}$.

MS (ESI) $\mathrm{m} / \mathrm{z}: \quad 1104.7[\mathrm{M}+6 \mathrm{H}]^{6+}, 1325.5[\mathrm{M}+5 \mathrm{H}]^{5+}, 1656.6[\mathrm{M}+4 \mathrm{H}]^{4+}$, deconvoluted 6622.3.

HR-MS (ESI) $m / z:$ calcd. for $\mathrm{C}_{299} \mathrm{H}_{448} \mathrm{~N}_{99} \mathrm{O}_{69} \mathrm{~S}_{3}[\mathrm{M}+7 \mathrm{H}]^{7+} 947.0545$, found 947.0532;

calcd. for $\mathrm{C}_{299} \mathrm{H}_{447} \mathrm{~N}_{99} \mathrm{O}_{69} \mathrm{~S}_{3}[\mathrm{M}+6 \mathrm{H}]^{6+} 1104.7290$, found 1104.7321;

calcd. for $\mathrm{C}_{299} \mathrm{H}_{446} \mathrm{~N}_{99} \mathrm{O}_{69} \mathrm{~S}_{3}[\mathrm{M}+5 \mathrm{H}]^{5+} 1325.4734$, found 1325.4758;

calcd. for $\mathrm{C}_{299} \mathrm{H}_{445} \mathrm{~N}_{99} \mathrm{O}_{69} \mathrm{~S}_{3}[\mathrm{M}+4 \mathrm{H}]^{4+} 1656.5899$, found 1656.5881 . 


\section{Experimental Part}

\section{PNA1/SybK}

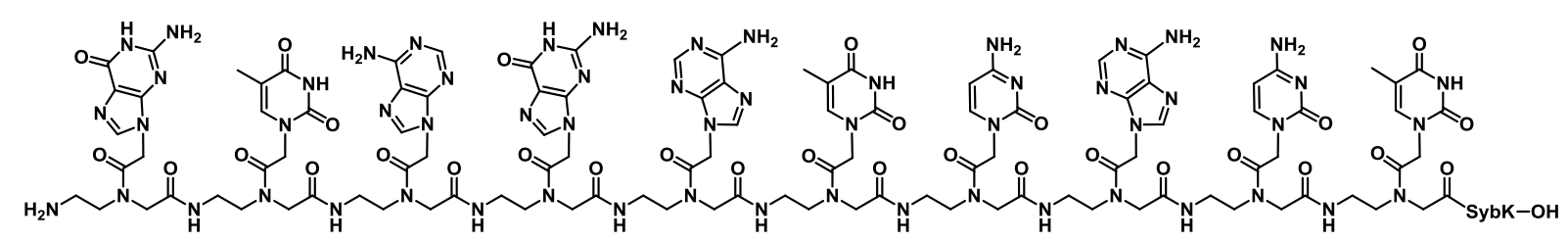

$\left[\mathrm{C}_{300} \mathrm{H}_{446} \mathrm{~N}_{100} \mathrm{O}_{67} \mathrm{~S}_{3}, 6621.68\right]$

UPLC (ACE Excel 2 column, RP-C18, $100 \mathrm{~mm} \times 2.1 \mathrm{~mm}, 2 \mu \mathrm{m}, 100 \AA$, gradient: $60-90 \%$ of solvent $B 1$ for $15 \mathrm{~min}): t_{R}=7.9 \mathrm{~min}$.

MS (ESI) $m / z: \quad 1104.6[\mathrm{M}+6 \mathrm{H}]^{6+}, 1325.3[\mathrm{M}+5 \mathrm{H}]^{5+}$, deconvoluted $6621.4[\mathrm{M}]$.

HR-MS (ESI) $m / z:$ calcd. for $\mathrm{C}_{300} \mathrm{H}_{452} \mathrm{~N}_{100} \mathrm{O}_{67} \mathrm{~S}_{3}[\mathrm{M}+6 \mathrm{H}]^{6+} 1104.5711$, found 1104.5814;

calcd. for $\mathrm{C}_{300} \mathrm{H}_{451} \mathrm{~N}_{100} \mathrm{O}_{67} \mathrm{~S}_{3}[\mathrm{M}+5 \mathrm{H}]^{5+} 1325.2838$,

found 1325.2857 .

\section{PNA1/SybTa}

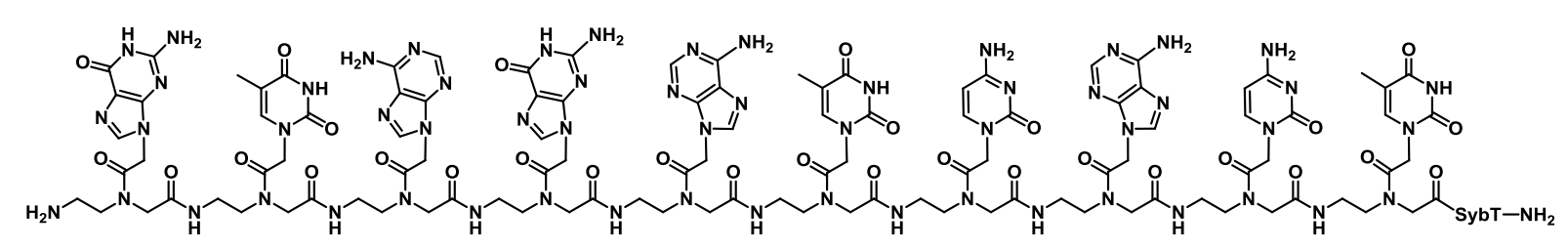

$\left[\mathrm{C}_{298} \mathrm{H}_{442} \mathrm{~N}_{100} \mathrm{O}_{67} \mathrm{~S}_{3}, 6593.63\right]$

UPLC (ACE Excel 2 column, RP-C18, $100 \mathrm{~mm} \times 2.1 \mathrm{~mm}, 2 \mu \mathrm{m}, 100 \AA$, gradient: $65-95 \%$ of solvent $B 1$ for $15 \mathrm{~min}): t_{R}=8.9 \mathrm{~min}$.

MS (ESI) m/z: $\quad 942.9[\mathrm{M}+7 \mathrm{H}]^{7+}, 1099.9[\mathrm{M}+6 \mathrm{H}]^{6+}, 1319.7[\mathrm{M}+5 \mathrm{H}]^{5+}$, $1649.3[\mathrm{M}+4 \mathrm{H}]^{4+}$, deconvoluted 6593.4 [M].

HR-MS (ESI) $m / z$ : calcd. for $\mathrm{C}_{298} \mathrm{H}_{449} \mathrm{~N}_{100} \mathrm{O}_{67} \mathrm{~S}_{3}[\mathrm{M}+7 \mathrm{H}]^{7+} 942.9146$, found 942.9113; 


\section{Experimental Part}

calcd. for $\mathrm{C}_{298} \mathrm{H}_{448} \mathrm{~N}_{100} \mathrm{O}_{67} \mathrm{~S}_{3}[\mathrm{M}+6 \mathrm{H}]^{6+} 1099.8992$, found 1099.9003;

calcd. for $\mathrm{C}_{298} \mathrm{H}_{447} \mathrm{~N}_{100} \mathrm{O}_{67} \mathrm{~S}_{3}[\mathrm{M}+5 \mathrm{H}]^{5+} 1319.6776$, found 1319.6800;

calcd. for $\mathrm{C}_{298} \mathrm{H}_{446} \mathrm{~N}_{100} \mathrm{O}_{67} \mathrm{~S}_{3}[\mathrm{M}+4 \mathrm{H}]^{4+} 1649.3451$, found 1649.3463 .

\section{PNA1/SybEa}

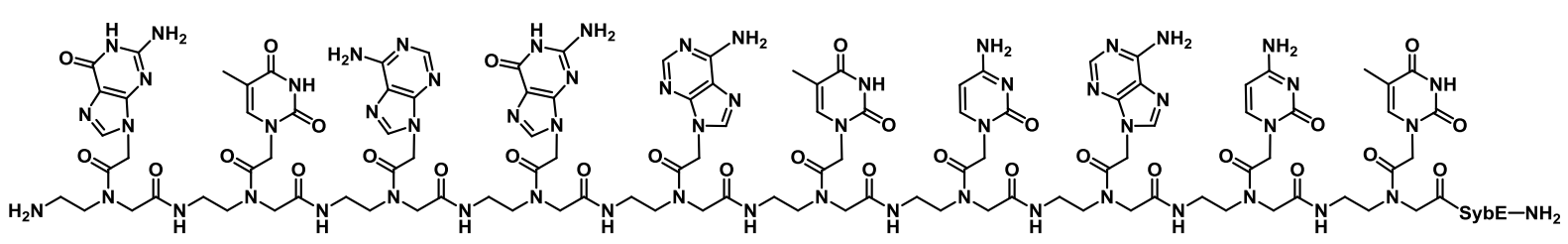

$\left[\mathrm{C}_{299} \mathrm{H}_{442} \mathrm{~N}_{100} \mathrm{O}_{68} \mathrm{~S}_{3}, 6621.64\right]$

UPLC (ACE Excel 2 column, RP-C18, $100 \mathrm{~mm} \times 2.1 \mathrm{~mm}, 2 \mu \mathrm{m}, 100 \AA$, gradient: $60-90 \%$ of solvent $B 1$ for $15 \mathrm{~min}): t_{R}=9.8 \mathrm{~min}$.

MS (ESI) m/z: $\quad 1104.6[\mathrm{M}+6 \mathrm{H}]^{6+}, 1325.3[\mathrm{M}+5 \mathrm{H}]^{5+}, 1656.1[\mathrm{M}+4 \mathrm{H}]^{4+}$, deconvoluted $6621.4[\mathrm{M}]$.

HR-MS (ESI) $m / z$ : calcd. for $\mathrm{C}_{299} \mathrm{H}_{448} \mathrm{~N}_{100} \mathrm{O}_{68} \mathrm{~S}_{3}[\mathrm{M}+6 \mathrm{H}]^{6+} 1104.5650$, found 1104.5688;

calcd. for $\mathrm{C}_{299} \mathrm{H}_{447} \mathrm{~N}_{100} \mathrm{O}_{68} \mathrm{~S}_{3}[\mathrm{M}+5 \mathrm{H}]^{5+} 1325.2766$, found 1325.2761. 


\section{PNA1/SybKa}

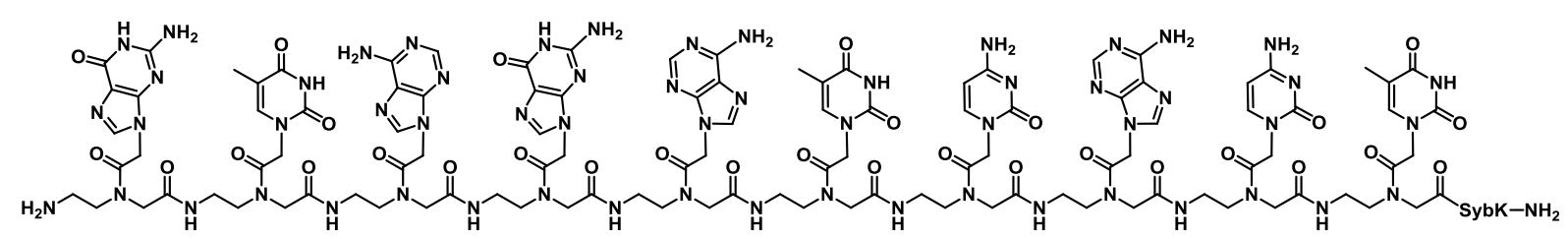

$\left[\mathrm{C}_{300} \mathrm{H}_{447} \mathrm{~N}_{101} \mathrm{O}_{66} \mathrm{~S}_{3}, 6620.70\right]$

UPLC (ACE Excel 2 column, RP-C18, $100 \mathrm{~mm} \times 2.1 \mathrm{~mm}, 2 \mu \mathrm{m}, 100 \AA$, gradient: $60-90 \%$ of solvent $B 1$ for $15 \mathrm{~min}): t_{\mathrm{R}}=8.2 \mathrm{~min}$.

MS (ESI) m/z: $\quad 1104.4[\mathrm{M}+6 \mathrm{H}]^{6+}, 1325.3[\mathrm{M}+5 \mathrm{H}]^{5+}, 1656.1[\mathrm{M}+4 \mathrm{H}]^{4+}$, deconvoluted $6620.4[\mathrm{M}]$.

HR-MS (ESI) m/z: calcd. for $\mathrm{C}_{300} \mathrm{H}_{453} \mathrm{~N}_{101} \mathrm{O}_{66} \mathrm{~S}_{3}[\mathrm{M}+6 \mathrm{H}]^{6+}$ 1104.4071, found 1104.4072;

calcd. for $\mathrm{C}_{300} \mathrm{H}_{452} \mathrm{~N}_{101} \mathrm{O}_{66} \mathrm{~S}_{3}[\mathrm{M}+5 \mathrm{H}]^{5+} 1325.0870$,

found 1325.0889;

calcd. for $\mathrm{C}_{300} \mathrm{H}_{451} \mathrm{~N}_{101} \mathrm{O}_{66} \mathrm{~S}_{3}[\mathrm{M}+4 \mathrm{H}]^{4+}$ 1656.1070, found 1656.1077 .

\subsubsection{2) PNA1/SxK Peptide}

\section{$\underline{\text { PNA1/SxK }}$}

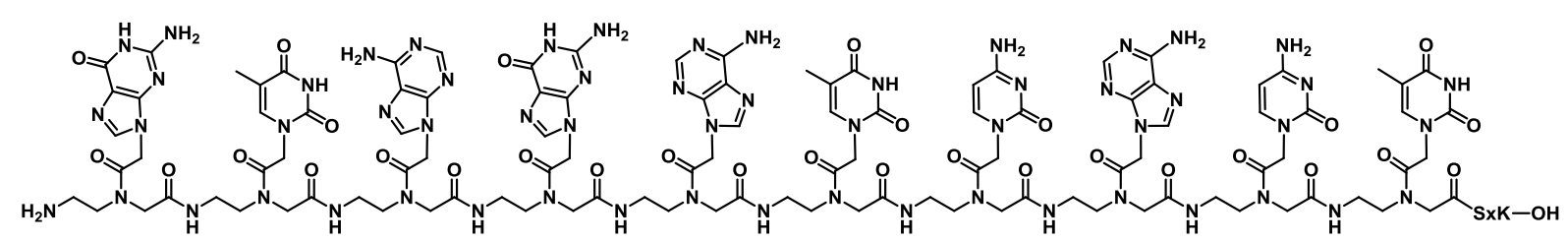

$\left[\mathrm{C}_{277} \mathrm{H}_{430} \mathrm{~N}_{102} \mathrm{O}_{69} \mathrm{~S}_{3}, 6389.31\right]$

UPLC (ACE Excel 2 column, RP-C18, $100 \mathrm{~mm} \times 2.1 \mathrm{~mm}, 2 \mu \mathrm{m}, 100 \AA$, gradient: $45-70 \%$ of solvent $B 1$ for $15 \mathrm{~min}): t_{R}=8.8 \mathrm{~min}$. 


\section{Experimental Part}

MS (ESI) m/z: $1065.9[\mathrm{M}+6 \mathrm{H}]^{6+}, 1278.9[\mathrm{M}+5 \mathrm{H}]^{5+}, 1598.3[\mathrm{M}+4 \mathrm{H}]^{4+}$, deconvoluted 6389.3 [M].

HR-MS (ESI) $m / z$ : calcd. for $\mathrm{C}_{277} \mathrm{H}_{436} \mathrm{~N}_{102} \mathrm{O}_{69} \mathrm{~S}_{3}[\mathrm{M}+6 \mathrm{H}]^{6+}$ 1065.8828,

found 1065.8835;

calcd. for $\mathrm{C}_{277} \mathrm{H}_{435} \mathrm{~N}_{102} \mathrm{O}_{69} \mathrm{~S}_{3}[\mathrm{M}+5 \mathrm{H}]^{5+} 1278.8579$,

found 1278.8592;

calcd. for $\mathrm{C}_{277} \mathrm{H}_{434} \mathrm{~N}_{102} \mathrm{O}_{69} \mathrm{~S}_{3}[\mathrm{M}+4 \mathrm{H}]^{4+} 1598.3206$,

found 1598,3185 .

\subsubsection{3) PNA2/SxY(a) Peptides}

\section{$\underline{\mathrm{PNA} 2 / \mathrm{S} \times \mathrm{G}}$}

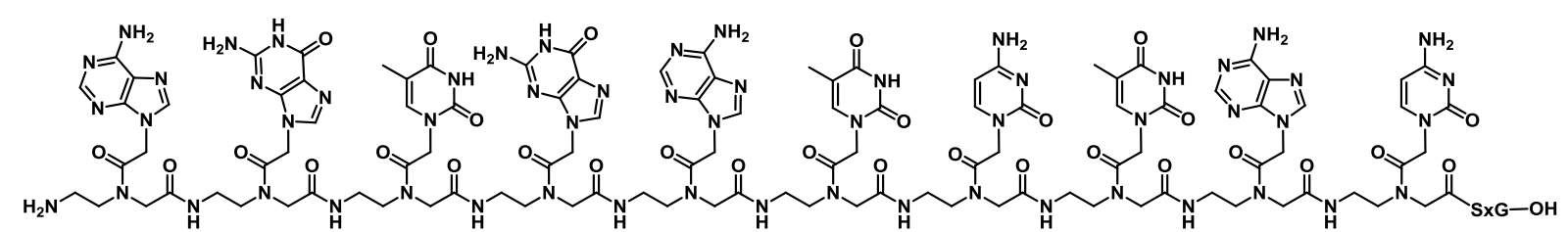

$\left[\mathrm{C}_{273} \mathrm{H}_{421} \mathrm{~N}_{101} \mathrm{O}_{69} \mathrm{~S}_{3}, 6318.19\right]$

UPLC (ACE Excel 2 column, RP-C18, $100 \mathrm{~mm} \times 2.1 \mathrm{~mm}, 2 \mu \mathrm{m}, 100 \AA$, gradient: $45-70 \%$ of solvent $B 1$ for $15 \mathrm{~min}): t_{\mathrm{R}}=9.9 \mathrm{~min}$.

MS (ESI) m/z: $1054.0[\mathrm{M}+6 \mathrm{H}]^{6+}, 1264.4[\mathrm{M}+5 \mathrm{H}]^{5+}, 1580.3[\mathrm{M}+4 \mathrm{H}]^{4+}$, deconvoluted $6318.2[\mathrm{M}]$.

HR-MS (ESI) m/z: calcd. for $\mathrm{C}_{273} \mathrm{H}_{427} \mathrm{~N}_{101} \mathrm{O}_{69} \mathrm{~S}_{3}[\mathrm{M}+6 \mathrm{H}]^{6+} 1054.0372$, found 1054.0387;

calcd. for $\mathrm{C}_{273} \mathrm{H}_{426} \mathrm{~N}_{101} \mathrm{O}_{69} \mathrm{~S}_{3}[\mathrm{M}+5 \mathrm{H}]^{5+} 1264.6432$,

found 1264.6500;

calcd. for $\mathrm{C}_{273} \mathrm{H}_{425} \mathrm{~N}_{101} \mathrm{O}_{69} \mathrm{~S}_{3}[\mathrm{M}+4 \mathrm{H}]^{4+} 1580.5522$, found 1580.5538 . 


\section{Experimental Part}

\section{PNA2/SXE}

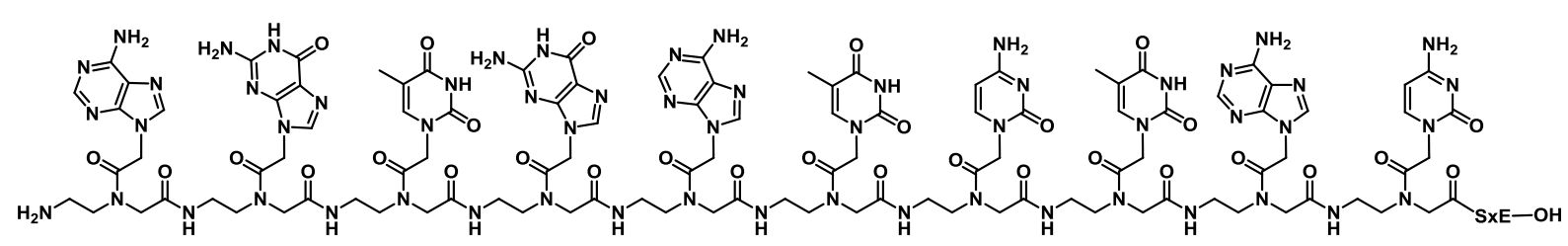

$\left[\mathrm{C}_{276} \mathrm{H}_{425} \mathrm{~N}_{101} \mathrm{O}_{71} \mathrm{~S}_{3}, 6390.25\right]$

UPLC (ACE Excel 2 column, RP-C18, $100 \mathrm{~mm} \times 2.1 \mathrm{~mm}, 2 \mu \mathrm{m}, 100 \AA$, gradient: $45-70 \%$ of solvent $B 1$ for $15 \mathrm{~min}$ ): $t_{R}=9.1 \mathrm{~min}$.

MS (ESI) m/z: $\quad 913.8[\mathrm{M}+7 \mathrm{H}]^{7+}, 1065.9[\mathrm{M}+6 \mathrm{H}]^{6+}, 1279.1[\mathrm{M}+5 \mathrm{H}]^{5+}$, $1598.6[\mathrm{M}+4 \mathrm{H}]^{4+}$, deconvoluted $6390.2[\mathrm{M}]$.

HR-MS (ESI) $m / z$ : calcd. for $\mathrm{C}_{276} \mathrm{H}_{431} \mathrm{~N}_{101} \mathrm{O}_{71} \mathrm{~S}_{3}[\mathrm{M}+6 \mathrm{H}]^{6+}$ 1066.0408, found 1066.0440;

calcd. for $\mathrm{C}_{276} \mathrm{H}_{430} \mathrm{~N}_{101} \mathrm{O}_{71} \mathrm{~S}_{3}[\mathrm{M}+5 \mathrm{H}]^{5+} 1279.0474$,

found 1279.0512 .

\section{PNA2/SxK}

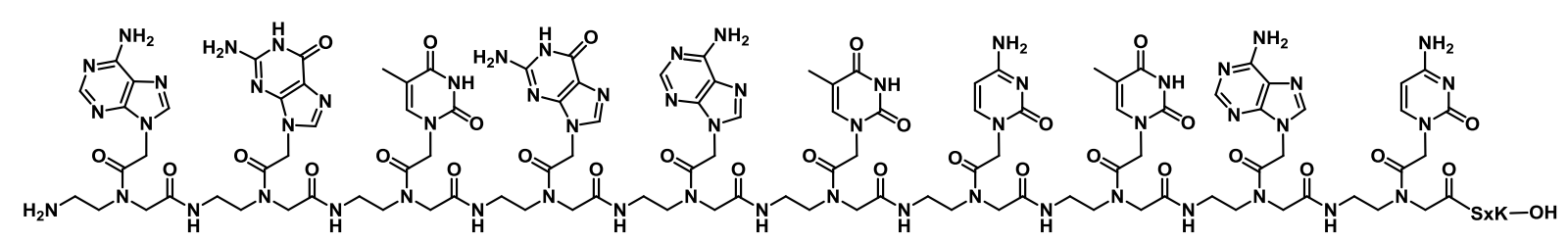

$\left[\mathrm{C}_{277} \mathrm{H}_{430} \mathrm{~N}_{102} \mathrm{O}_{69} \mathrm{~S}_{3}, 6389.31\right]$

UPLC (ACE Excel 2 column, RP-C18, $100 \mathrm{~mm} \times 2.1 \mathrm{~mm}, 2 \mu \mathrm{m}, 100 \AA$, gradient: $45-70 \%$ of solvent $B 1$ for $15 \mathrm{~min}): t_{R}=9.0 \mathrm{~min}$.

MS (ESI) $m / z: \quad 799.7[\mathrm{M}+8 \mathrm{H}]^{8+}, 913.8[\mathrm{M}+7 \mathrm{H}]^{7+}, 1065.8[\mathrm{M}+6 \mathrm{H}]^{6+}$, $1278.9[\mathrm{M}+5 \mathrm{H}]^{5+}$, deconvoluted $6389.3[\mathrm{M}]$. 


\section{Experimental Part}

HR-MS (ESI) $m / z$ : calcd. for $\mathrm{C}_{277} \mathrm{H}_{436} \mathrm{~N}_{102} \mathrm{O}_{69} \mathrm{~S}_{3}[\mathrm{M}+6 \mathrm{H}]^{6+} 1065.8828$, found 1065.8837;

calcd. for $\mathrm{C}_{277} \mathrm{H}_{435} \mathrm{~N}_{102} \mathrm{O}_{69} \mathrm{~S}_{3}[\mathrm{M}+5 \mathrm{H}]^{5+} 1278.8579$, found 1278.8564 .

\section{PNA2/SxGa}

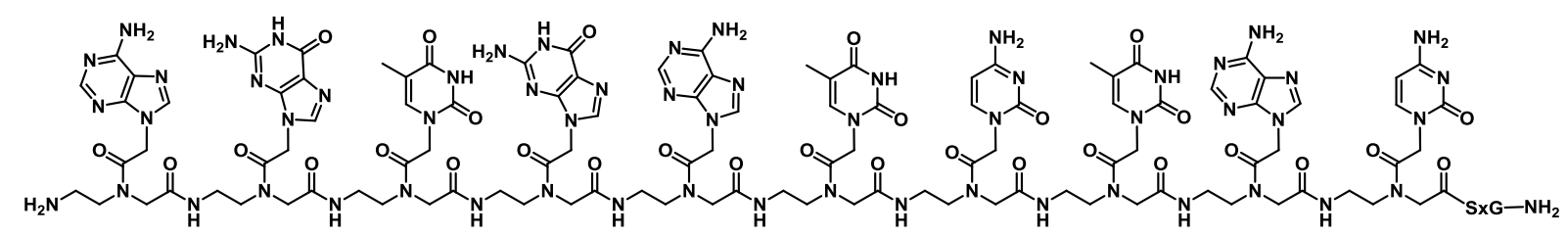

$\left[\mathrm{C}_{273} \mathrm{H}_{422} \mathrm{~N}_{102} \mathrm{O}_{68} \mathrm{~S}_{3}, 6317.21\right]$

UPLC (ACE Excel 2 column, RP-C18, $100 \mathrm{~mm} \times 2.1 \mathrm{~mm}, 2 \mu \mathrm{m}, 100 \AA$, gradient: $45-70 \%$ of solvent $B 1$ for $15 \mathrm{~min}): t_{R}=10.3 \mathrm{~min}$.

MS (ESI) m/z: $\quad 1053.9[\mathrm{M}+6 \mathrm{H}]^{6+}, 1264.4[\mathrm{M}+5 \mathrm{H}]^{5+}, 1580.3[\mathrm{M}+4 \mathrm{H}]^{4+}$, deconvoluted $6317.2[\mathrm{M}]$.

HR-MS (ESI) $m / z:$ calcd. for $\mathrm{C}_{273} \mathrm{H}_{428} \mathrm{~N}_{102} \mathrm{O}_{68} \mathrm{~S}_{3}[\mathrm{M}+6 \mathrm{H}]^{6+} 1053.8732$, found 1053.8737;

calcd. for $\mathrm{C}_{273} \mathrm{H}_{427} \mathrm{~N}_{102} \mathrm{O}_{68} \mathrm{~S}_{3}[\mathrm{M}+5 \mathrm{H}]^{5+} 1264.4464$, found 1264.4470;

calcd. for $\mathrm{C}_{273} \mathrm{H}_{426} \mathrm{~N}_{102} \mathrm{O}_{68} \mathrm{~S}_{3}[\mathrm{M}+4 \mathrm{H}]^{4+} 1580.3062$, found 1580.3079 . 


\section{PNA2/SxEa}

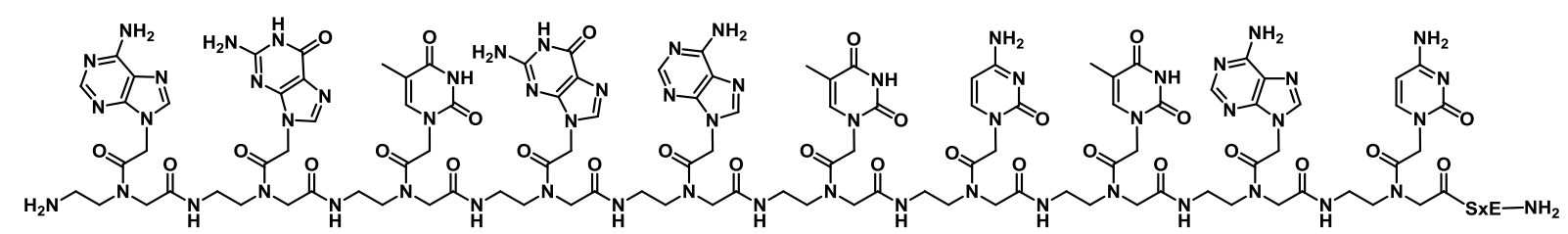

$\left[\mathrm{C}_{276} \mathrm{H}_{426} \mathrm{~N}_{102} \mathrm{O}_{70} \mathrm{~S}_{3}, 6389.27\right]$

UPLC (ACE Excel 2 column, RP-C18, $100 \mathrm{~mm} \times 2.1 \mathrm{~mm}, 2 \mu \mathrm{m}, 100 \AA$, gradient: $45-70 \%$ of solvent $B 1$ for $15 \mathrm{~min}): t_{R}=9.9 \mathrm{~min}$.

MS (ESI) $m / z: \quad 1065.9[\mathrm{M}+6 \mathrm{H}]^{6+}, 1278.6[\mathrm{M}+5 \mathrm{H}]^{5+}, 1598.3[\mathrm{M}+4 \mathrm{H}]^{4+}$, deconvoluted $6389.2[\mathrm{M}]$.

HR-MS (ESI) $m / z$ : calcd. for $\mathrm{C}_{276} \mathrm{H}_{432} \mathrm{~N}_{102} \mathrm{O}_{70} \mathrm{~S}_{3}[\mathrm{M}+6 \mathrm{H}]^{6+}$ 1065.8767, found 1065.8779;

calcd. for $\mathrm{C}_{276} \mathrm{H}_{431} \mathrm{~N}_{102} \mathrm{O}_{70} \mathrm{~S}_{3}[\mathrm{M}+5 \mathrm{H}]^{5+} 1278.8506$,

found 1278.8522;

calcd. for $\mathrm{C}_{276} \mathrm{H}_{430} \mathrm{~N}_{102} \mathrm{O}_{70} \mathrm{~S}_{3}[\mathrm{M}+4 \mathrm{H}]^{4+} 1598.3115$,

found 1598.3121 .

\section{PNA2/SXKa}

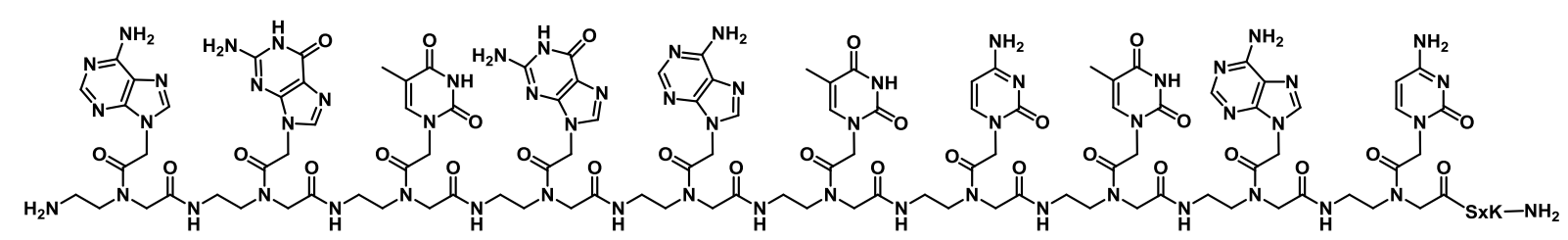

$\left[\mathrm{C}_{277} \mathrm{H}_{431} \mathrm{~N}_{103} \mathrm{O}_{68} \mathrm{~S}_{3}, 6388.33\right]$

UPLC (ACE Excel 2 column, RP-C18, $100 \mathrm{~mm} \times 2.1 \mathrm{~mm}, 2 \mu \mathrm{m}, 100 \AA$, gradient: $45-70 \%$ of solvent $B 1$ for $15 \mathrm{~min}$ ): $t_{R}=9.1 \mathrm{~min}$.

MS (ESI) $\mathrm{m} / \mathrm{z}: \quad 913.6[\mathrm{M}+7 \mathrm{H}]^{7+}, 1065.7[\mathrm{M}+6 \mathrm{H}]^{6+}, 1278.7[\mathrm{M}+5 \mathrm{H}]^{5+}$, deconvoluted $6388.3[\mathrm{M}]$. 


\section{Experimental Part}

HR-MS (ESI) $m / z$ : calcd. for $\mathrm{C}_{277} \mathrm{H}_{438} \mathrm{~N}_{103} \mathrm{O}_{68} \mathrm{~S}_{3}[\mathrm{M}+7 \mathrm{H}]^{7+} 913.6172$, found 913.6145;

calcd. for $\mathrm{C}_{277} \mathrm{H}_{437} \mathrm{~N}_{103} \mathrm{O}_{68} \mathrm{~S}_{3}[\mathrm{M}+6 \mathrm{H}]^{6+} 1065.7188$, found 1065.7213;

calcd. for $\mathrm{C}_{277} \mathrm{H}_{436} \mathrm{~N}_{103} \mathrm{O}_{68} \mathrm{~S}_{3}[\mathrm{M}+5 \mathrm{H}]^{5+} 1278.6611$, found 1278.6716 .

\subsubsection{4) PNA3/SxY(a) Peptides}

\section{PNA3/SxG}

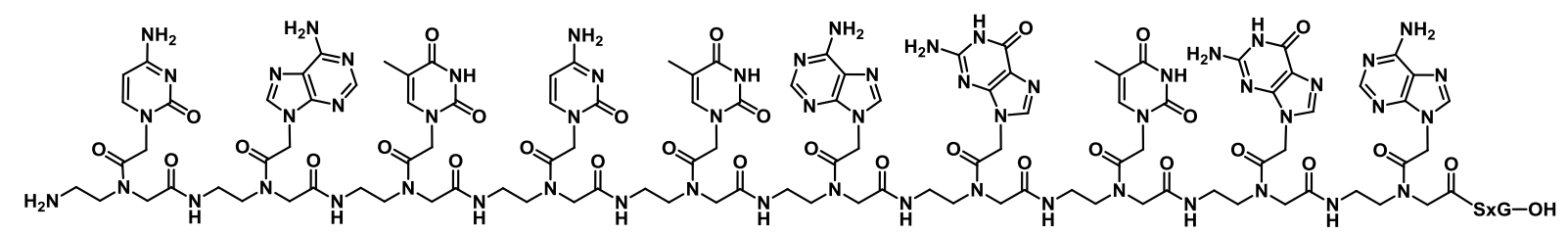

$\left[\mathrm{C}_{273} \mathrm{H}_{421} \mathrm{~N}_{101} \mathrm{O}_{69} \mathrm{~S}_{3}, 6318.19\right]$

UPLC (ACE Excel 2 column, RP-C18, $100 \mathrm{~mm} \times 2.1 \mathrm{~mm}, 2 \mu \mathrm{m}, 100 \AA$, gradient: $45-70 \%$ of solvent $B 1$ for $15 \mathrm{~min}): t_{R}=10.0 \mathrm{~min}$.

MS (ESI) m/z: $1053.9[\mathrm{M}+6 \mathrm{H}]^{6+}, 1264.7[\mathrm{M}+5 \mathrm{H}]^{5+}, 1580.6[\mathrm{M}+4 \mathrm{H}]^{4+}$, deconvoluted $6318.2[\mathrm{M}]$.

HR-MS (ESI) $m / z:$ calcd. for $\mathrm{C}_{273} \mathrm{H}_{427} \mathrm{~N}_{101} \mathrm{O}_{69} \mathrm{~S}_{3}[\mathrm{M}+6 \mathrm{H}]^{6+} 1054.0372$, found 1054.0395;

calcd. for $\mathrm{C}_{273} \mathrm{H}_{426} \mathrm{~N}_{101} \mathrm{O}_{69} \mathrm{~S}_{3}[\mathrm{M}+5 \mathrm{H}]^{5+} 1264.6432$, found 1264.6470;

calcd. for $\mathrm{C}_{273} \mathrm{H}_{425} \mathrm{~N}_{101} \mathrm{O}_{69} \mathrm{~S}_{3}[\mathrm{M}+4 \mathrm{H}]^{4+} 1580.5522$, found 1580.5537 . 


\section{Experimental Part}

\section{PNA3/SXE}

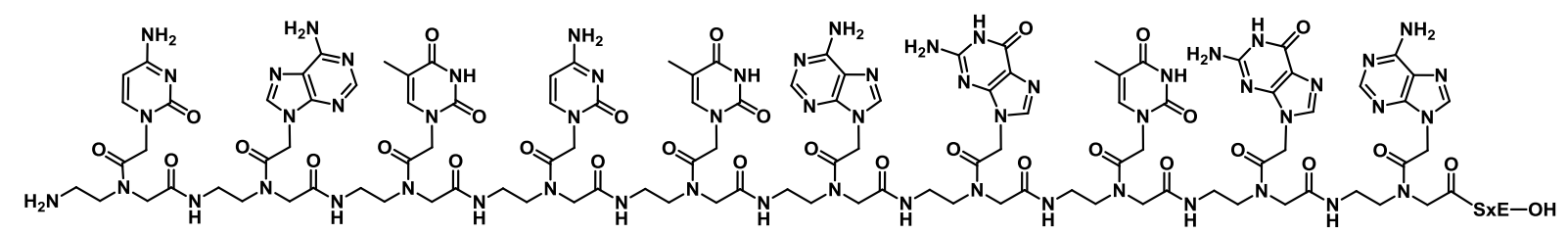

$\left[\mathrm{C}_{276} \mathrm{H}_{425} \mathrm{~N}_{101} \mathrm{O}_{71} \mathrm{~S}_{3}, 6390.25\right]$

UPLC (ACE Excel 2 column, RP-C18, $100 \mathrm{~mm} \times 2.1 \mathrm{~mm}, 2 \mu \mathrm{m}, 100 \AA$, gradient: $45-70 \%$ of solvent $B 1$ for $15 \mathrm{~min}): t_{R}=9.3 \mathrm{~min}$.

MS (ESI) $m / z: \quad 913.9[\mathrm{M}+7 \mathrm{H}]^{7+}, 1066.1[\mathrm{M}+6 \mathrm{H}]^{6+}, 1278.7[\mathrm{M}+5 \mathrm{H}]^{5+}$, deconvoluted $6390.2[\mathrm{M}]$.

HR-MS (ESI) $m / z$ : calcd. for $\mathrm{C}_{276} \mathrm{H}_{431} \mathrm{~N}_{101} \mathrm{O}_{71} \mathrm{~S}_{3}[\mathrm{M}+6 \mathrm{H}]^{6+}$ 1066.0408, found 1066.0416;

calcd. for $\mathrm{C}_{276} \mathrm{H}_{430} \mathrm{~N}_{101} \mathrm{O}_{71} \mathrm{~S}_{3}[\mathrm{M}+5 \mathrm{H}]^{5+} 1279.0474$, found 1279.0484 .

\section{PNA3/SxK}

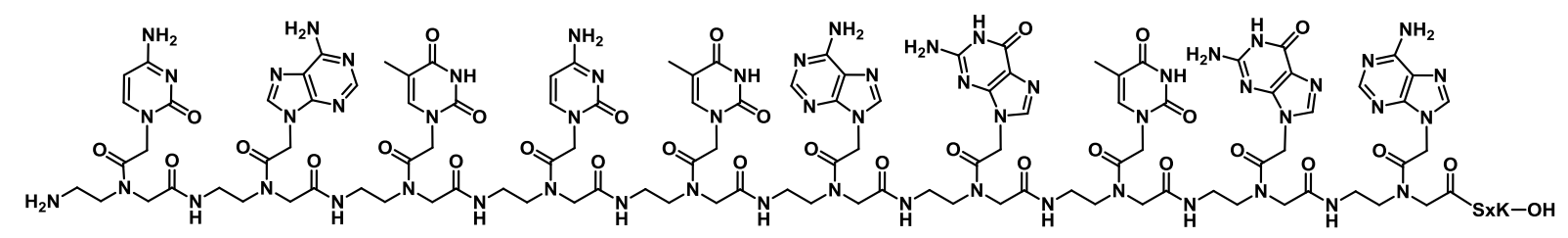

$\left[\mathrm{C}_{277} \mathrm{H}_{430} \mathrm{~N}_{102} \mathrm{O}_{69} \mathrm{~S}_{3}, 6389.31\right]$

UPLC (ACE Excel 2 column, RP-C18, $100 \mathrm{~mm} \times 2.1 \mathrm{~mm}, 2 \mu \mathrm{m}, 100 \AA$, gradient: $45-70 \%$ of solvent $B 1$ for $15 \mathrm{~min}): t_{R}=9.0 \mathrm{~min}$.

MS (ESI) $m / z: \quad 799.7[\mathrm{M}+8 \mathrm{H}]^{8+}, 913.8[\mathrm{M}+7 \mathrm{H}]^{7+}, 1065.8[\mathrm{M}+6 \mathrm{H}]^{6+}$, $1278.9[\mathrm{M}+5 \mathrm{H}]^{5+}$, deconvoluted $6389.3[\mathrm{M}]$.

HR-MS (ESI) m/z: calcd. for $\mathrm{C}_{277} \mathrm{H}_{438} \mathrm{~N}_{102} \mathrm{O}_{69} \mathrm{~S}_{3}[\mathrm{M}+8 \mathrm{H}]^{8+} 799.6639$, found 799.6655; 


\section{Experimental Part}

calcd. for $\mathrm{C}_{277} \mathrm{H}_{437} \mathrm{~N}_{102} \mathrm{O}_{69} \mathrm{~S}_{3}[\mathrm{M}+7 \mathrm{H}]^{7+} 913.7577$, found 913.7606;

calcd. for $\mathrm{C}_{277} \mathrm{H}_{436} \mathrm{~N}_{102} \mathrm{O}_{69} \mathrm{~S}_{3}[\mathrm{M}+6 \mathrm{H}]^{6+}$ 1065.8828, found 1065.8847;

calcd. for $\mathrm{C}_{277} \mathrm{H}_{435} \mathrm{~N}_{102} \mathrm{O}_{69} \mathrm{~S}_{3}[\mathrm{M}+5 \mathrm{H}]^{5+} 1278.8579$, found 1278.8611 .

\section{PNA3/SxGa}

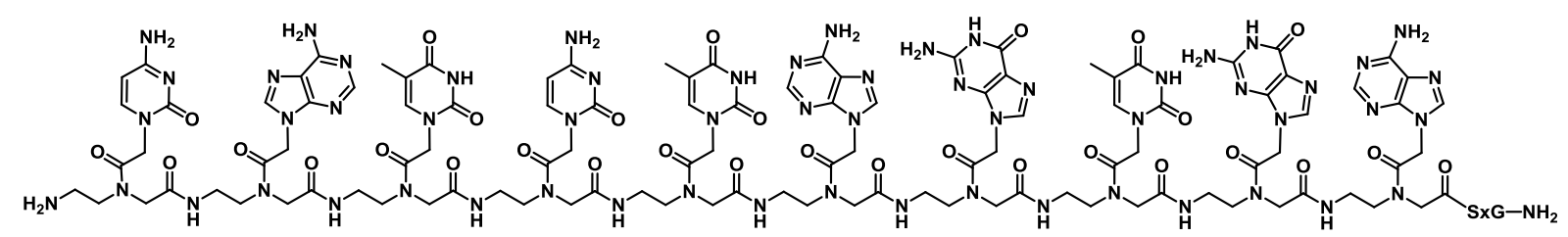

$\left[\mathrm{C}_{273} \mathrm{H}_{422} \mathrm{~N}_{102} \mathrm{O}_{68} \mathrm{~S}_{3}, 6317.21\right]$

UPLC (ACE Excel 2 column, RP-C18, $100 \mathrm{~mm} \times 2.1 \mathrm{~mm}, 2 \mu \mathrm{m}, 100 \AA$, gradient: $45-70 \%$ of solvent $B 1$ for $15 \mathrm{~min}): t_{R}=10.4 \mathrm{~min}$.

MS (ESI) m/z: $1053.9[\mathrm{M}+6 \mathrm{H}]^{6+}, 1264.4[\mathrm{M}+5 \mathrm{H}]^{5+}, 1580.3[\mathrm{M}+4 \mathrm{H}]^{4+}$, deconvoluted $6317.2[\mathrm{M}]$.

HR-MS (ESI) $m / z$ : calcd. for $\mathrm{C}_{273} \mathrm{H}_{428} \mathrm{~N}_{102} \mathrm{O}_{68} \mathrm{~S}_{3}[\mathrm{M}+6 \mathrm{H}]^{6+}$ 1053.8732, found 1053.8739;

calcd. for $\mathrm{C}_{273} \mathrm{H}_{427} \mathrm{~N}_{102} \mathrm{O}_{68} \mathrm{~S}_{3}[\mathrm{M}+5 \mathrm{H}]^{5+} 1264.4464$, found 1264.4474;

calcd. for $\mathrm{C}_{273} \mathrm{H}_{426} \mathrm{~N}_{102} \mathrm{O}_{68} \mathrm{~S}_{3}[\mathrm{M}+4 \mathrm{H}]^{4+} 1580.3062$, found 1580.3062 . 


\section{$\underline{\text { PNA3/SXEa }}$}

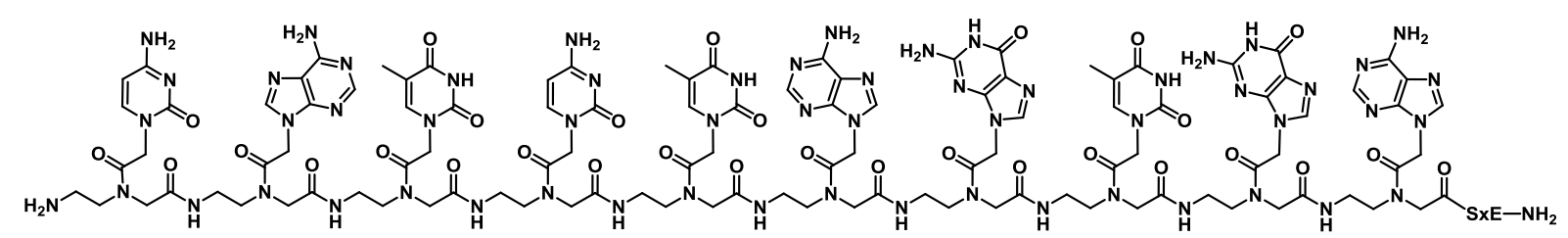

$\left[\mathrm{C}_{276} \mathrm{H}_{426} \mathrm{~N}_{102} \mathrm{O}_{70} \mathrm{~S}_{3}, 6389.27\right]$

UPLC (ACE Excel 2 column, RP-C18, $100 \mathrm{~mm} \times 2.1 \mathrm{~mm}, 2 \mu \mathrm{m}, 100 \AA$, gradient: $45-70 \%$ of solvent $B 1$ for $15 \mathrm{~min}): t_{R}=9.8 \mathrm{~min}$.

MS (ESI) $m / z: \quad 1065.9[\mathrm{M}+6 \mathrm{H}]^{6+}, 1278.6[\mathrm{M}+5 \mathrm{H}]^{5+}, 1598.3[\mathrm{M}+4 \mathrm{H}]^{4+}$, deconvoluted $6389.2[\mathrm{M}]$.

HR-MS (ESI) $m / z$ : calcd. for $\mathrm{C}_{276} \mathrm{H}_{432} \mathrm{~N}_{102} \mathrm{O}_{70} \mathrm{~S}_{3}[\mathrm{M}+6 \mathrm{H}]^{6+}$ 1065.8767, found 1065.8766;

calcd. for $\mathrm{C}_{276} \mathrm{H}_{431} \mathrm{~N}_{102} \mathrm{O}_{70} \mathrm{~S}_{3}[\mathrm{M}+5 \mathrm{H}]^{5+} 1278.8506$,

found 1278.8511;

calcd. for $\mathrm{C}_{276} \mathrm{H}_{430} \mathrm{~N}_{102} \mathrm{O}_{70} \mathrm{~S}_{3}[\mathrm{M}+4 \mathrm{H}]^{4+} 1598.3115$,

found 1598.3120 .

\section{PNA3/SXKa}

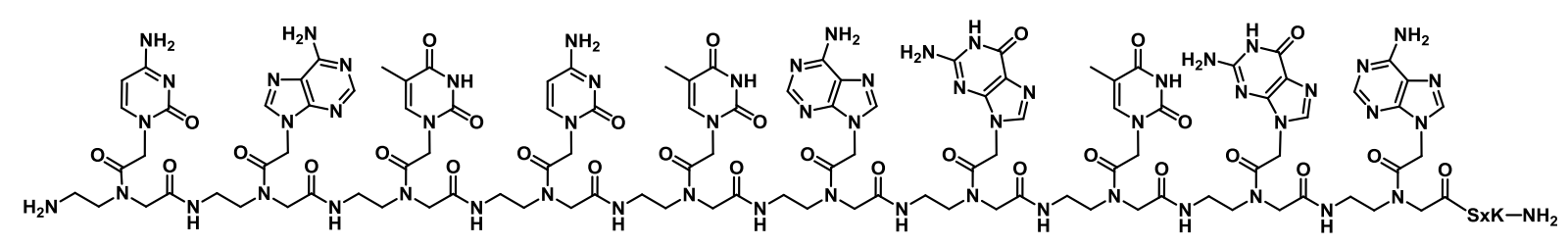

$\left[\mathrm{C}_{277} \mathrm{H}_{431} \mathrm{~N}_{103} \mathrm{O}_{68} \mathrm{~S}_{3}, 6388.33\right]$

UPLC (ACE Excel 2 column, RP-C18, $100 \mathrm{~mm} \times 2.1 \mathrm{~mm}, 2 \mu \mathrm{m}, 100 \AA$, gradient: $45-70 \%$ of solvent $B 1$ for $15 \mathrm{~min}$ ): $t_{R}=9.2 \mathrm{~min}$.

MS (ESI) m/z: $1065.7[\mathrm{M}+6 \mathrm{H}]^{6+}, 1278.6[\mathrm{M}+5 \mathrm{H}]^{5+}, 1598.1[\mathrm{M}+4 \mathrm{H}]^{4+}$, deconvoluted 6388.3 [M]. 


\section{Experimental Part}

HR-MS (ESI) $m / z$ : calcd. for $\mathrm{C}_{277} \mathrm{H}_{437} \mathrm{~N}_{103} \mathrm{O}_{68} \mathrm{~S}_{3}[\mathrm{M}+6 \mathrm{H}]^{6+}$ 1065.7188, found 1065.7191;

calcd. for $\mathrm{C}_{277} \mathrm{H}_{436} \mathrm{~N}_{103} \mathrm{O}_{68} \mathrm{~S}_{3}[\mathrm{M}+5 \mathrm{H}]^{5+} 1278.6611$, found 1278.6637;

calcd. for $\mathrm{C}_{277} \mathrm{H}_{435} \mathrm{~N}_{103} \mathrm{O}_{68} \mathrm{~S}_{3}[\mathrm{M}+4 \mathrm{H}]^{4+} 1598.0746$, found 1598.0733 .

\subsection{5) E3/K3-LR/TMD Peptide Hybrids}

Synthesis of peptides was conducted according to Section 5.4.5 and after cleavage from the resin, peptides were dissolved in methanol/TFE/formic acid $(4: 1: 1, v / v / v)$ for mass spectrometric analysis.

\subsubsection{1) (i)E3/SybX(a) Peptides and Cys-E3/SybT}

\section{E3/SybT}

$$
\text { H-G-(EIAALEK)3-RKYWWKNLKMMIILGVICAIILIIIIVYFST-OH }
$$

$$
\left[\mathrm{C}_{288} \mathrm{H}_{473} \mathrm{~N}_{65} \mathrm{O}_{71} \mathrm{~S}_{3}, 6078.52\right]
$$

UPLC (ACE Excel 2 column, RP-C18, $100 \mathrm{~mm} \times 2.1 \mathrm{~mm}, 2 \mu \mathrm{m}, 100 \AA$, gradient: $70-90 \%$ of solvent $B 1$ for $15 \mathrm{~min}): t_{R}=10.0 \mathrm{~min}$.

MS (ESI) $m / z: \quad 869.2[\mathrm{M}+7 \mathrm{H}]^{7+}, 1013.9[\mathrm{M}+6 \mathrm{H}]^{6+}, 1216.5[\mathrm{M}+5 \mathrm{H}]^{5+}$, $1520.4[\mathrm{M}+4 \mathrm{H}]^{4+}$, deconvoluted $6078.5[\mathrm{M}]$.

HR-MS (ESI) $\mathrm{m} / \mathrm{z}: \quad$ calcd. for $\mathrm{C}_{288} \mathrm{H}_{480} \mathrm{~N}_{65} \mathrm{O}_{71} \mathrm{~S}_{3}[\mathrm{M}+7 \mathrm{H}]^{7+} 869.3597$, found 869.3597 ;

calcd. for $\mathrm{C}_{288} \mathrm{H}_{479} \mathrm{~N}_{65} \mathrm{O}_{71} \mathrm{~S}_{3}[\mathrm{M}+6 \mathrm{H}]^{6+} 1014.0851$, found 1014.0848;

calcd. for $\mathrm{C}_{288} \mathrm{H}_{478} \mathrm{~N}_{65} \mathrm{O}_{71} \mathrm{~S}_{3}[\mathrm{M}+5 \mathrm{H}]^{5+}$ 1216.7006, found 1216.7006; 


\section{Experimental Part}

calcd. for $\mathrm{C}_{288} \mathrm{H}_{477} \mathrm{~N}_{65} \mathrm{O}_{71} \mathrm{~S}_{3}[\mathrm{M}+4 \mathrm{H}]^{4+} 1520.6239$, found 1520.6232 .

\section{$\underline{\text { iE3/SybT }}$}

H-G-(KELAAIE) ${ }_{3}$-RKYWWKNLKMMIILGVICAIILIIIIVYFST-OH

$\left[\mathrm{C}_{288} \mathrm{H}_{473} \mathrm{~N}_{65} \mathrm{O}_{71} \mathrm{~S}_{3}, 6078.52\right]$

UPLC ( $A C E$ Excel 2 column, RP-C18, $100 \mathrm{~mm} \times 2.1 \mathrm{~mm}, 2 \mu \mathrm{m}, 100 \AA$, two-step gradient: $70-85 \%$ and $85-95 \%$ of solvent B1 for 10 min ( $1^{\text {st }}$ step) and 5 min ( $2^{\text {nd }}$ step)): $t_{\mathrm{R}}=13.0 \mathrm{~min}$.

MS (ESI) m/z: $\quad 1014.1[\mathrm{M}+6 \mathrm{H}]^{6+}, 1216.7[\mathrm{M}+5 \mathrm{H}]^{5+}, 1520.6[\mathrm{M}+4 \mathrm{H}]^{4+}$, deconvoluted $6078.5[\mathrm{M}]$.

HR-MS (ESI) $m / z$ : calcd. for $\mathrm{C}_{288} \mathrm{H}_{479} \mathrm{~N}_{65} \mathrm{O}_{71} \mathrm{~S}_{3}[\mathrm{M}+6 \mathrm{H}]^{6+}$ 1014.0851, found 1014.0825;

calcd. for $\mathrm{C}_{288} \mathrm{H}_{478} \mathrm{~N}_{65} \mathrm{O}_{71} \mathrm{~S}_{3}[\mathrm{M}+5 \mathrm{H}]^{5+} 1216.7006$, found 1216.6974;

calcd. for $\mathrm{C}_{288} \mathrm{H}_{477} \mathrm{~N}_{65} \mathrm{O}_{71} \mathrm{~S}_{3}[\mathrm{M}+4 \mathrm{H}]^{4+} 1520.6239$, found 1520.6203 .

\section{Cys-E3/SybT}

H-CG-(EIAALEK) ${ }_{3}$-RKYWWKNLKMMIILGVICAIILIIIIVYFST-OH

$\left[\mathrm{C}_{291} \mathrm{H}_{478} \mathrm{~N}_{66} \mathrm{O}_{72} \mathrm{~S}_{4}, 6181.66\right]$

MS (ESI) $m / z$ :

$884.1[\mathrm{M}+7 \mathrm{H}]^{7+}, \quad 1031.3[\mathrm{M}+6 \mathrm{H}]^{6+}$, $1237.4[\mathrm{M}+5 \mathrm{H}]^{5+}$, $1546.5[\mathrm{M}+4 \mathrm{H}]^{4+}$, deconvoluted $6181.5[\mathrm{M}]$.

HR-MS (ESI) $m / z$ : calcd. for $\mathrm{C}_{291} \mathrm{H}_{485} \mathrm{~N}_{66} \mathrm{O}_{72} \mathrm{~S}_{4}[\mathrm{M}+7 \mathrm{H}]^{7+} 884.0752$, found 884.0753;

calcd. for $\mathrm{C}_{291} \mathrm{H}_{484} \mathrm{~N}_{66} \mathrm{O}_{72} \mathrm{~S}_{4}[\mathrm{M}+6 \mathrm{H}]^{6+} 1031.2532$, found 1031.2539; 


\section{Experimental Part}

calcd. for $\mathrm{C}_{291} \mathrm{H}_{483} \mathrm{~N}_{66} \mathrm{O}_{72} \mathrm{~S}_{4}[\mathrm{M}+5 \mathrm{H}]^{5+} 1237.3024$, found 1237.3032;

calcd. for $\mathrm{C}_{291} \mathrm{H}_{482} \mathrm{~N}_{66} \mathrm{O}_{72} \mathrm{~S}_{4}[\mathrm{M}+4 \mathrm{H}]^{4+}$ 1546.3762, found 1546.3764 .

\section{E3/SybE}

H-G-(EIAALEK)3-RKYWWKNLKMMIILGVICAIILIIIIVYFSE-OH

$\left[\mathrm{C}_{289} \mathrm{H}_{473} \mathrm{~N}_{65} \mathrm{O}_{72} \mathrm{~S}_{3}, 6106.53\right]$

UPLC (ACE Excel 2 column, RP-C18, $100 \mathrm{~mm} \times 2.1 \mathrm{~mm}, 2 \mu \mathrm{m}, 100 \AA$, gradient: $70-90 \%$ of solvent $B 1$ for $15 \mathrm{~min}): t_{R}=7.5 \mathrm{~min}$.

MS (ESI) m/z: $\quad 1018.7[\mathrm{M}+6 \mathrm{H}]^{6+}, 1222.3[\mathrm{M}+5 \mathrm{H}]^{5+}, 1527.6[\mathrm{M}+4 \mathrm{H}]^{4+}$, deconvoluted $6106.5[\mathrm{M}]$.

HR-MS (ESI) $m / z$ : calcd. for $\mathrm{C}_{289} \mathrm{H}_{479} \mathrm{~N}_{65} \mathrm{O}_{72} \mathrm{~S}_{3}[\mathrm{M}+6 \mathrm{H}]^{6+}$ 1018.7509, found 1018.7493;

calcd. for $\mathrm{C}_{289} \mathrm{H}_{478} \mathrm{~N}_{65} \mathrm{O}_{72} \mathrm{~S}_{3}[\mathrm{M}+5 \mathrm{H}]^{5+} 1222.2996$, found 1222.3000;

\section{E3/SybK}

\section{H-G-(EIAALEK)з-RKYWWKNLKMMIILGVICAIILIIIIVYFSK-OH}

$\left[\mathrm{C}_{290} \mathrm{H}_{478} \mathrm{~N}_{66} \mathrm{O}_{70} \mathrm{~S}_{3}, 6105.59\right]$

UPLC (ACE Excel 2 column, RP-C18, $100 \mathrm{~mm} \times 2.1 \mathrm{~mm}, 2 \mu \mathrm{m}, 100 \AA$, gradient: $60-90 \%$ of solvent $B 1$ for $15 \mathrm{~min}): t_{R}=10.2 \mathrm{~min}$.

MS (ESI) m/z: $\quad 873.2[\mathrm{M}+7 \mathrm{H}]^{7+}, 1018.4[\mathrm{M}+6 \mathrm{H}]^{6+}, 1221.9[\mathrm{M}+5 \mathrm{H}]^{5+}$, deconvoluted $6105.5[\mathrm{M}]$.

HR-MS (ESI) m/z: calcd. for $\mathrm{C}_{290} \mathrm{H}_{485} \mathrm{~N}_{66} \mathrm{O}_{70} \mathrm{~S}_{3}[\mathrm{M}+7 \mathrm{H}]^{7+} 873.2236$, found 873.2220; 


\section{Experimental Part}

calcd. for $\mathrm{C}_{290} \mathrm{H}_{484} \mathrm{~N}_{66} \mathrm{O}_{70} \mathrm{~S}_{3}[\mathrm{M}+6 \mathrm{H}]^{6+} 1018.5934$, found 1018.5934;

calcd. for $\mathrm{C}_{290} \mathrm{H}_{483} \mathrm{~N}_{66} \mathrm{O}_{70} \mathrm{~S}_{3}[\mathrm{M}+5 \mathrm{H}]^{5+} 1222.1101$, found 1222.1115 .

\section{E3/SybTa}

H-G-(EIAALEK) ${ }_{3}-\mathrm{RKYWWKNLKMMIILGVICAIILIIIIVYFST-NH_{2 }}$

$\left[\mathrm{C}_{288} \mathrm{H}_{474} \mathrm{~N}_{66} \mathrm{O}_{70} \mathrm{~S}_{3}, 6077.53\right]$

UPLC (ACE Excel 2 column, RP-C18, $100 \mathrm{~mm} \times 2.1 \mathrm{~mm}, 2 \mu \mathrm{m}, 100 \AA$, gradient: $70-90 \%$ of solvent $\mathrm{B} 1$ for $15 \mathrm{~min}$ ): $t_{\mathrm{R}}=11.3 \mathrm{~min}$.

MS (ESI) m/z: $\quad 869.1[\mathrm{M}+7 \mathrm{H}]^{7+}, 1013.8[\mathrm{M}+6 \mathrm{H}]^{6+}, 1216.5[\mathrm{M}+5 \mathrm{H}]^{5+}$, $1520.1[\mathrm{M}+4 \mathrm{H}]^{4+}$, deconvoluted $6077.5[\mathrm{M}]$.

HR-MS (ESI) $m / z$ : calcd. for $\mathrm{C}_{288} \mathrm{H}_{480} \mathrm{~N}_{66} \mathrm{O}_{70} \mathrm{~S}_{3}[\mathrm{M}+6 \mathrm{H}]^{6+} 1013.9211$, found 1013.9206;

calcd. for $\mathrm{C}_{288} \mathrm{H}_{479} \mathrm{~N}_{66} \mathrm{O}_{70} \mathrm{~S}_{3}[\mathrm{M}+5 \mathrm{H}]^{5+} 1216.5038$, found 1216.5037;

calcd. for $\mathrm{C}_{288} \mathrm{H}_{478} \mathrm{~N}_{66} \mathrm{O}_{70} \mathrm{~S}_{3}[\mathrm{M}+4 \mathrm{H}]^{4+} 1520.3779$, found 1520.3775 .

\section{E3/SybEa}

H-G-(EIAALEK)3-RKYWWKNLKMMIILGVICAIILIIIIVYFSE-NH

$\left[\mathrm{C}_{289} \mathrm{H}_{474} \mathrm{~N}_{66} \mathrm{O}_{71} \mathrm{~S}_{3}, 6105.54\right]$

UPLC (ACE Excel 2 column, RP-C18, $100 \mathrm{~mm} \times 2.1 \mathrm{~mm}, 2 \mu \mathrm{m}, 100 \AA$, gradient: $70-90 \%$ of solvent $B 1$ for $15 \mathrm{~min}): t_{R}=8.4 \mathrm{~min}$.

MS (ESI) m/z: $\quad 873.2[\mathrm{M}+7 \mathrm{H}]^{7+}, 1018.6[\mathrm{M}+6 \mathrm{H}]^{6+}, 1221.9[\mathrm{M}+5 \mathrm{H}]^{5+}$, $1527.4[\mathrm{M}+4 \mathrm{H}]^{4+}$, deconvoluted $6105.5[\mathrm{M}]$. 


\section{Experimental Part}

HR-MS (ESI) $m / z$ : calcd. for $\mathrm{C}_{289} \mathrm{H}_{481} \mathrm{~N}_{66} \mathrm{O}_{71} \mathrm{~S}_{3}[\mathrm{M}+7 \mathrm{H}]^{7+} 873.2184$, found 873.2159;

calcd. for $\mathrm{C}_{289} \mathrm{H}_{480} \mathrm{~N}_{66} \mathrm{O}_{71} \mathrm{~S}_{3}[\mathrm{M}+6 \mathrm{H}]^{6+}$ 1018.5869, found 1018.5859;

calcd. for $\mathrm{C}_{289} \mathrm{H}_{479} \mathrm{~N}_{66} \mathrm{O}_{71} \mathrm{~S}_{3}[\mathrm{M}+5 \mathrm{H}]^{5+} 1222.1028$, found 1222.1035;

calcd. for $\mathrm{C}_{289} \mathrm{H}_{478} \mathrm{~N}_{66} \mathrm{O}_{71} \mathrm{~S}_{3}[\mathrm{M}+4 \mathrm{H}]^{4+} 1527.3762$, found 1527.3762 .

\section{E3/SybKa}

H-G-(EIAALEK)3-RKYWWKNLKMMIILGVICAIILIIIIVYFSK-NH

$\left[\mathrm{C}_{290} \mathrm{H}_{479} \mathrm{~N}_{67} \mathrm{O}_{69} \mathrm{~S}_{3}, 6104,60\right]$

UPLC (ACE Excel 2 column, RP-C18, $100 \mathrm{~mm} \times 2.1 \mathrm{~mm}, 2 \mu \mathrm{m}, 100 \AA$ A gradient: $60-90 \%$ of solvent $B 1$ for $15 \mathrm{~min}): t_{R}=10.8 \mathrm{~min}$.

MS (ESI) $m / z: \quad 872.9[\mathrm{M}+7 \mathrm{H}]^{7+}, 1018.3[\mathrm{M}+6 \mathrm{H}]^{6+}, 1221.7[\mathrm{M}+5 \mathrm{H}]^{5+}$, $1526.9[\mathrm{M}+4 \mathrm{H}]^{4+}$, deconvoluted 6104.5 [M].

HR-MS (ESI) $m / z$ : calcd. for $\mathrm{C}_{290} \mathrm{H}_{486} \mathrm{~N}_{67} \mathrm{O}_{69} \mathrm{~S}_{3}[\mathrm{M}+7 \mathrm{H}]^{7+} 873.0830$, found 873.0813;

calcd. for $\mathrm{C}_{290} \mathrm{H}_{485} \mathrm{~N}_{67} \mathrm{O}_{69} \mathrm{~S}_{3}[\mathrm{M}+6 \mathrm{H}]^{6+} 1018.4289$, found 1018.4285;

calcd. for $\mathrm{C}_{290} \mathrm{H}_{484} \mathrm{~N}_{67} \mathrm{O}_{69} \mathrm{~S}_{3}[\mathrm{M}+5 \mathrm{H}]^{5+} 1221.9133$, found 1221.9133;

calcd. for $\mathrm{C}_{290} \mathrm{H}_{483} \mathrm{~N}_{67} \mathrm{O}_{69} \mathrm{~S}_{3}[\mathrm{M}+4 \mathrm{H}]^{4+} 1527.1398$, found 1527.1382 . 


\subsubsection{2) $\quad \mathrm{K} 3 / \mathrm{SxY}(\mathrm{a})$ Peptides}

$\underline{\mathrm{K} 3 / \mathrm{S} x \mathrm{G}}$

H-WWG-(KIAALKE) ${ }_{3}$-QSKARRKKIMIIICCVILGIIIASTIGGIFG-OH

$\left[\mathrm{C}_{279} \mathrm{H}_{479} \mathrm{~N}_{73} \mathrm{O}_{66} \mathrm{~S}_{3}, 6008.53\right]$

UPLC (ACE Excel 2 column, RP-C18, $100 \mathrm{~mm} \times 2.1 \mathrm{~mm}, 2 \mu \mathrm{m}, 100 \AA$, gradient: $45-70 \%$ of solvent $B 1$ for $15 \mathrm{~min}): t_{R}=10.9 \mathrm{~min}$.

MS (ESI) $\mathrm{m} / \mathrm{z}: \quad 859.2[\mathrm{M}+7 \mathrm{H}]^{7+}, 1002.3[\mathrm{M}+6 \mathrm{H}]^{6+}, 1202.5[\mathrm{M}+5 \mathrm{H}]^{5+}$, $1502.9[\mathrm{M}+4 \mathrm{H}]^{4+}$, deconvoluted $6008.5[\mathrm{M}]$.

HR-MS (ESI) $m / z$ : calcd. for $\mathrm{C}_{279} \mathrm{H}_{486} \mathrm{~N}_{73} \mathrm{O}_{66} \mathrm{~S}_{3}[\mathrm{M}+7 \mathrm{H}]^{7+} 859.3735$, found 859.3713;

calcd. for $\mathrm{C}_{279} \mathrm{H}_{485} \mathrm{~N}_{73} \mathrm{O}_{66} \mathrm{~S}_{3}[\mathrm{M}+6 \mathrm{H}]^{6+} 1002.4345$, found 1002.4339;

calcd. for $\mathrm{C}_{279} \mathrm{H}_{484} \mathrm{~N}_{73} \mathrm{O}_{66} \mathrm{~S}_{3}[\mathrm{M}+5 \mathrm{H}]^{5+} 1202.7200$, found 1202.7192;

calcd. for $\mathrm{C}_{279} \mathrm{H}_{483} \mathrm{~N}_{73} \mathrm{O}_{66} \mathrm{~S}_{3}[\mathrm{M}+4 \mathrm{H}]^{4+}$ 1503.1481, found 1503.1473 .

$\underline{\mathrm{K} 3 / \mathrm{S} x \mathrm{E}}$

H-WWG-(KIAALKE) ${ }_{3}$-QSKARRKKIMIIICCVILGIIIASTIGGIFE-OH

$\left[\mathrm{C}_{282} \mathrm{H}_{483} \mathrm{~N}_{73} \mathrm{O}_{68} \mathrm{~S}_{3}, 6080.59\right]$

UPLC (ACE Excel 2 column, RP-C18, $100 \mathrm{~mm} \times 2.1 \mathrm{~mm}, 2 \mu \mathrm{m}, 100 \AA$, gradient: $45-70 \%$ of solvent $B 1$ for $15 \mathrm{~min}$ ): $t_{R}=10.2 \mathrm{~min}$.

MS (ESI) $m / z: \quad 761.1[\mathrm{M}+8 \mathrm{H}]^{8+}, 869.5[\mathrm{M}+7 \mathrm{H}]^{7+}, 1014.3[\mathrm{M}+6 \mathrm{H}]^{6+}$, $1217.1[\mathrm{M}+5 \mathrm{H}]^{5+}, 1521.2[\mathrm{M}+4 \mathrm{H}]^{4+}$, deconvoluted $6080.6[\mathrm{M}]$.

HR-MS (ESI) $m / z$ : calcd. for $\mathrm{C}_{282} \mathrm{H}_{491} \mathrm{~N}_{73} \mathrm{O}_{68} \mathrm{~S}_{3}[\mathrm{M}+8 \mathrm{H}]^{8+} 761.0786$, found 761.0786;

calcd. for $\mathrm{C}_{282} \mathrm{H}_{490} \mathrm{~N}_{73} \mathrm{O}_{68} \mathrm{~S}_{3}[\mathrm{M}+7 \mathrm{H}]^{7+} 869.6622$, 


\section{Experimental Part}

found 869.6622 ;

calcd. for $\mathrm{C}_{282} \mathrm{H}_{489} \mathrm{~N}_{73} \mathrm{O}_{68} \mathrm{~S}_{3}[\mathrm{M}+6 \mathrm{H}]^{6+} 1014.4380$,

found 1014.4381;

calcd. for $\mathrm{C}_{282} \mathrm{H}_{488} \mathrm{~N}_{73} \mathrm{O}_{68} \mathrm{~S}_{3}[\mathrm{M}+5 \mathrm{H}]^{5+} 1217.1242$, found 1271.1241.

$\underline{K 3 / S x K}$

H-WWG-(KIAALKE) ${ }_{3}$-QSKARRKKIMIIICCVILGIIIASTIGGIFK-OH

$\left[\mathrm{C}_{283} \mathrm{H}_{488} \mathrm{~N}_{74} \mathrm{O}_{66} \mathrm{~S}_{3}, 6079.65\right]$

UPLC (ACE Excel 2 column, RP-C18, $100 \mathrm{~mm} \times 2.1 \mathrm{~mm}, 2 \mu \mathrm{m}, 100 \AA$, gradient: $45-70 \%$ of solvent $B 1$ for $15 \mathrm{~min}): t_{R}=9.3 \mathrm{~min}$.

MS (ESI) $m / z: \quad 869.5[\mathrm{M}+7 \mathrm{H}]^{7+}, 1014.3[\mathrm{M}+6 \mathrm{H}]^{6+}, 1216.7[\mathrm{M}+5 \mathrm{H}]^{5+}$, $1520.9[\mathrm{M}+4 \mathrm{H}]^{4+}$, deconvoluted $6079.6[\mathrm{M}]$.

HR-MS (ESI) $m / z$ : calcd. for $\mathrm{C}_{283} \mathrm{H}_{495} \mathrm{~N}_{74} \mathrm{O}_{66} \mathrm{~S}_{3}[\mathrm{M}+7 \mathrm{H}]^{7+} 869.5268$, found 869.5245 ;

calcd. for $\mathrm{C}_{283} \mathrm{H}_{494} \mathrm{~N}_{74} \mathrm{O}_{66} \mathrm{~S}_{3}[\mathrm{M}+6 \mathrm{H}]^{6+}$ 1014.2801, found 1014.2791;

calcd. for $\mathrm{C}_{283} \mathrm{H}_{493} \mathrm{~N}_{74} \mathrm{O}_{66} \mathrm{~S}_{3}[\mathrm{M}+5 \mathrm{H}]^{5+} 1216.9347$, found 1216.9345;

calcd. for $\mathrm{C}_{283} \mathrm{H}_{492} \mathrm{~N}_{74} \mathrm{O}_{66} \mathrm{~S}_{3}[\mathrm{M}+4 \mathrm{H}]^{4+} 1520.9165$, found 1520.9131.

$\underline{\mathrm{K} 3 / \mathrm{SxGa}}$

H-WWG-(KIAALKE)3-QSKARRKKIMIIICCVILGIIIASTIGGIFG-NH ${ }_{2}$

$\left[\mathrm{C}_{279} \mathrm{H}_{480} \mathrm{~N}_{74} \mathrm{O}_{65} \mathrm{~S}_{3}, 6007.54\right]$

UPLC (ACE Excel 2 column, RP-C18, $100 \mathrm{~mm} \times 2.1 \mathrm{~mm}, 2 \mu \mathrm{m}, 100 \AA$, gradient: $45-70 \%$ of solvent $B 1$ for $15 \mathrm{~min}): t_{R}=11.4 \mathrm{~min}$. 
MS (ESI) $m / z: \quad 859.2[\mathrm{M}+7 \mathrm{H}]^{7+}, 1002.1[\mathrm{M}+6 \mathrm{H}]^{6+}, 1202.5[\mathrm{M}+5 \mathrm{H}]^{5+}$, $1502.9[\mathrm{M}+4 \mathrm{H}]]^{4+}$, deconvoluted $6007.6[\mathrm{M}]$.

HR-MS (ESI) $\mathrm{m} / z$ : calcd. for $\mathrm{C}_{279} \mathrm{H}_{487} \mathrm{~N}_{74} \mathrm{O}_{65} \mathrm{~S}_{3}[\mathrm{M}+7 \mathrm{H}]^{7+}$ 859.2329, found 859.2314;

calcd. for $\mathrm{C}_{279} \mathrm{H}_{486} \mathrm{~N}_{74} \mathrm{O}_{65} \mathrm{~S}_{3}[\mathrm{M}+6 \mathrm{H}]^{6+}$ 1002.2705, found 1002.2695;

calcd. for $\mathrm{C}_{279} \mathrm{H}_{485} \mathrm{~N}_{74} \mathrm{O}_{65} \mathrm{~S}_{3}[\mathrm{M}+5 \mathrm{H}]^{5+} 1202.5231$, found 1202.5229;

calcd. for $\mathrm{C}_{279} \mathrm{H}_{484} \mathrm{~N}_{74} \mathrm{O}_{65} \mathrm{~S}_{3}[\mathrm{M}+4 \mathrm{H}]^{4+} 1502.9021$, found 1502.8964 .

\section{$\underline{\mathrm{K} 3 / \mathrm{SxEa}}$}

H-WWG-(KIAALKE) 3 -QSKARRKKIMIIICCVILGIIIASTIGGIFE-NH ${ }_{2}$

$\left[\mathrm{C}_{282} \mathrm{H}_{484} \mathrm{~N}_{74} \mathrm{O}_{67} \mathrm{~S}_{3}, 6079.61\right]$

UPLC (ACE Excel 2 column, RP-C18, $100 \mathrm{~mm} \times 2.1 \mathrm{~mm}, 2 \mu \mathrm{m}, 100 \AA$, gradient: $45-70 \%$ of solvent $B 1$ for $15 \mathrm{~min}$ ): $t_{R}=10.6 \mathrm{~min}$.

MS (ESI) m/z: $\quad 761.0[\mathrm{M}+8 \mathrm{H}]^{8+}, 869.4[\mathrm{M}+7 \mathrm{H}]^{7+}, 1014.1[\mathrm{M}+6 \mathrm{H}]^{6+}$, $1216.9[\mathrm{M}+5 \mathrm{H}]^{5+}$, deconvoluted 6079.6 [M].

HR-MS (ESI) $m / z$ : calcd. for $\mathrm{C}_{282} \mathrm{H}_{492} \mathrm{~N}_{74} \mathrm{O}_{67} \mathrm{~S}_{3}[\mathrm{M}+8 \mathrm{H}]^{8+} 760.9573$, found 760.9540 ;

calcd. for $\mathrm{C}_{282} \mathrm{H}_{491} \mathrm{~N}_{74} \mathrm{O}_{67} \mathrm{~S}_{3}[\mathrm{M}+7 \mathrm{H}]^{7+}$ 869.5216, found 869.5200;

calcd. for $\mathrm{C}_{282} \mathrm{H}_{490} \mathrm{~N}_{74} \mathrm{O}_{67} \mathrm{~S}_{3}[\mathrm{M}+6 \mathrm{H}]^{6+} 1014.2740$, found 1014.2732;

calcd. for $\mathrm{C}_{282} \mathrm{H}_{489} \mathrm{~N}_{74} \mathrm{O}_{67} \mathrm{~S}_{3}[\mathrm{M}+5 \mathrm{H}]^{5+} 1216.9269$, found 1216.9269 . 


\section{Experimental Part}

$\underline{\mathrm{K} 3 / \mathrm{SxKa}}$

$$
\begin{aligned}
& \text { H-WWG-(KIAALKE) }{ }_{3} \text {-QSKARRKKIMIIICCVILGIIIASTIGGIFK- } \mathrm{NH}_{2} \\
& {\left[\mathrm{C}_{283} \mathrm{H}_{489} \mathrm{~N}_{75} \mathrm{O}_{65} \mathrm{~S}_{3}, 6078.67\right]}
\end{aligned}
$$

UPLC (ACE Excel 2 column, RP-C18, $100 \mathrm{~mm} \times 2.1 \mathrm{~mm}, 2 \mu \mathrm{m}, 100 \AA$, gradient: $45-70 \%$ of solvent $B 1$ for $15 \mathrm{~min}$ ): $t_{R}=10.1 \mathrm{~min}$.

MS (ESI) $m / z: \quad 676.4[\mathrm{M}+9 \mathrm{H}]^{9+}, 760.7[\mathrm{M}+8 \mathrm{H}]^{8+}, 869.4[\mathrm{M}+7 \mathrm{H}]^{7+}$, $1013.9[\mathrm{M}+6 \mathrm{H}]^{6+}, 1216.7[\mathrm{M}+5 \mathrm{H}]^{5+}, 1520.7[\mathrm{M}+4 \mathrm{H}]^{4+}$, deconvoluted 6078.7 [M].

HR-MS (ESI) $\mathrm{m} / z$ : calcd. for $\mathrm{C}_{283} \mathrm{H}_{497} \mathrm{~N}_{75} \mathrm{O}_{65} \mathrm{~S}_{3}[\mathrm{M}+8 \mathrm{H}]^{8+} 760.8389$, found 760.8400 ;

calcd. for $\mathrm{C}_{283} \mathrm{H}_{496} \mathrm{~N}_{75} \mathrm{O}_{65} \mathrm{~S}_{3}[\mathrm{M}+7 \mathrm{H}]^{7+} 869.3863$, found 869.3864;

calcd. for $\mathrm{C}_{283} \mathrm{H}_{495} \mathrm{~N}_{75} \mathrm{O}_{65} \mathrm{~S}_{3}[\mathrm{M}+6 \mathrm{H}]^{6+}$ 1014.1161, found 1014.1162;

calcd. for $\mathrm{C}_{283} \mathrm{H}_{494} \mathrm{~N}_{75} \mathrm{O}_{65} \mathrm{~S}_{3}[\mathrm{M}+5 \mathrm{H}]^{5+} 1216.7379$, found 1216.7369 . 


\section{$\underline{\text { Appendix }}$}

\section{6) Appendix}

Solid Phase Peptide Synthesis: Fmoc-Deprotection

. i

Solid Phase Peptide Synthesis: Active Ester Formation...........................i

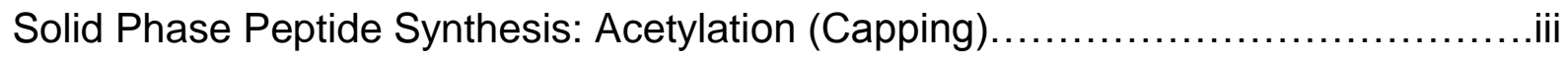

Solid Phase Peptide Synthesis: Cleavage of the Peptide from the Resin..............iv

Solid Phase Peptide Synthesis: Scavenger Reactions..........................

Solid Phase Peptide Synthesis: Aspartimide Formation..........................vi

Solid Phase Peptide Synthesis: Racemisation Reactions...........................vii

Comparison of Native and Artificial Sequences.................................ii

Chromatography Results of PNA-LR/TMD Peptide Hybrids ...........................

Chromatography Results of E3/K3-LR/TMD Peptide Hybrids.....................

Loss of Lipid Material During Proteoliposome Preparation and Size Exclusion

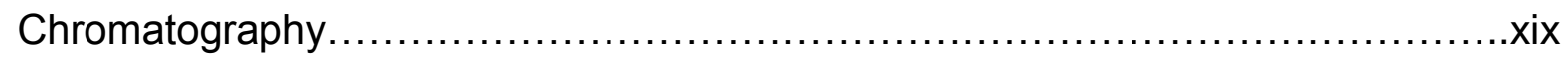

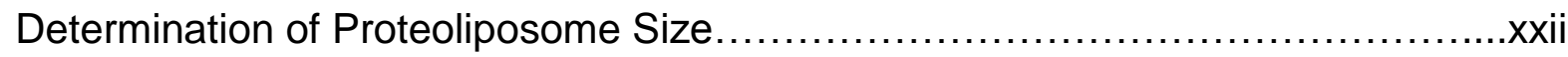




\section{$\underline{\text { Appendix }}$}

\section{Solid Phase Peptide Synthesis: Fmoc-Deprotection}
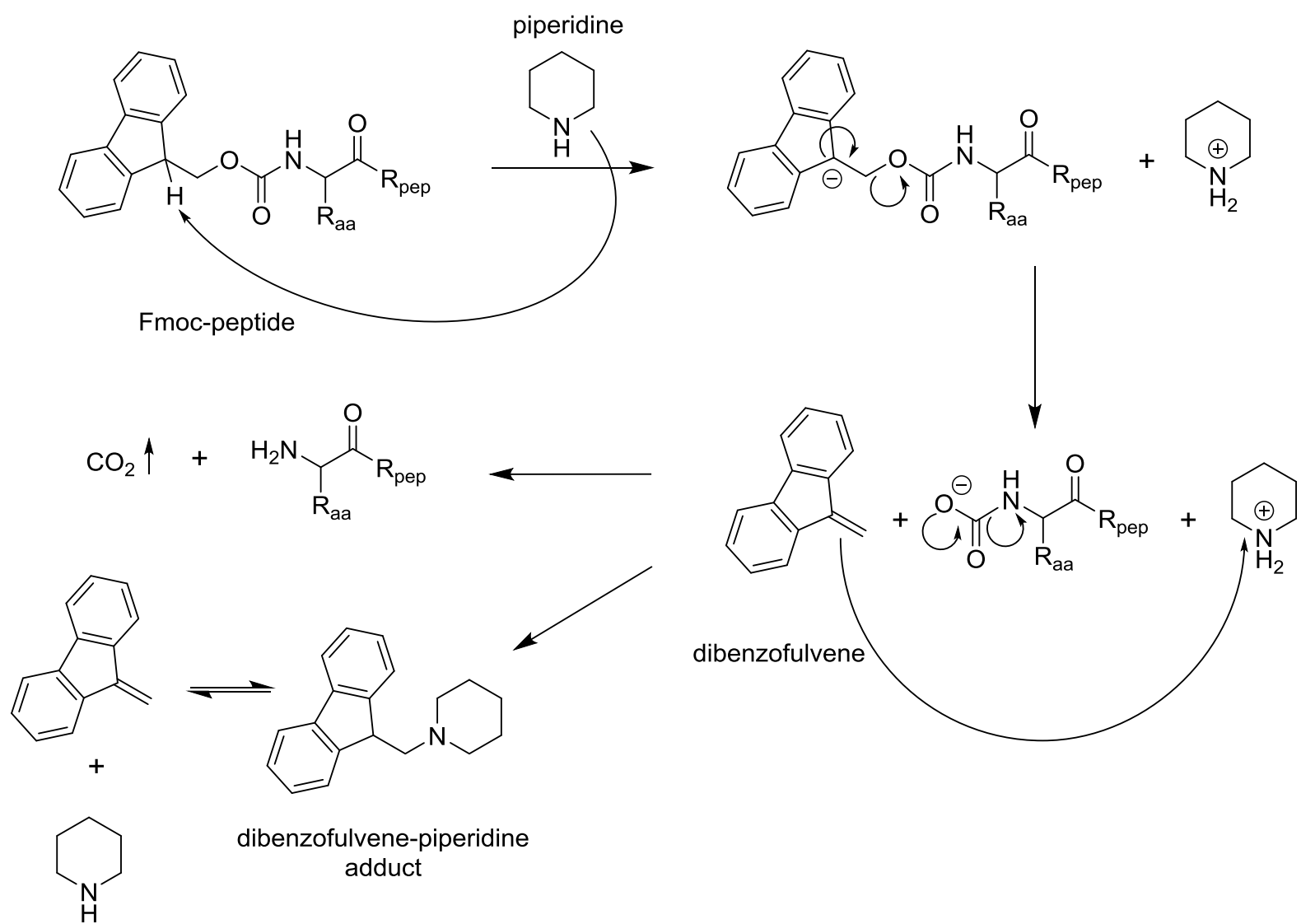

Figure A-1 Fmoc-deprotection through piperidine. The deprotection product can be monitored by means of UV-spectroscopy. Thus, it is possible to detect the effectiveness of the deprotection reaction (based on ref. 159). 


\section{Solid Phase Peptide Synthesis: Active Ester Formation}

A

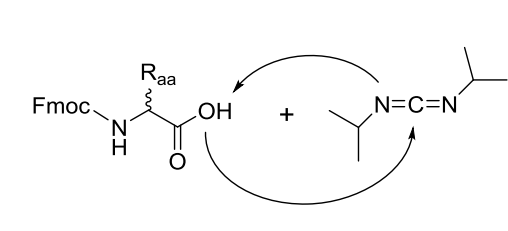

Fmoc-aa

DIC

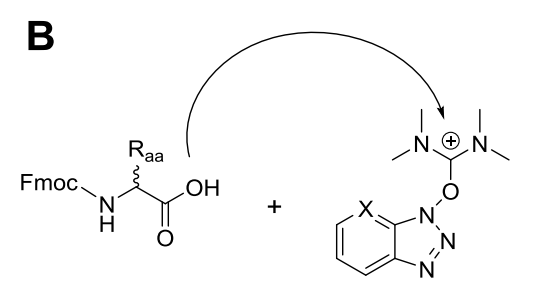

Fmoc-aa

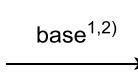

1) DIPEA 1) DIPEA
2) DIPEA/lutidine

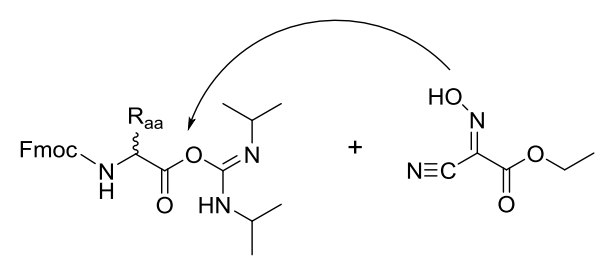

O-acylurea intermadiate oxyma

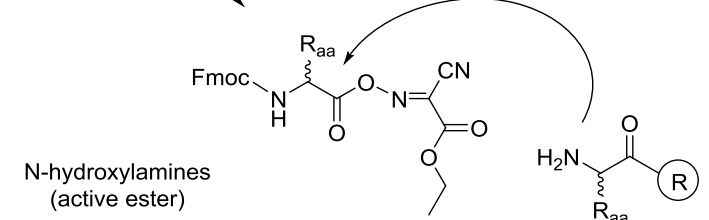

(active ester)
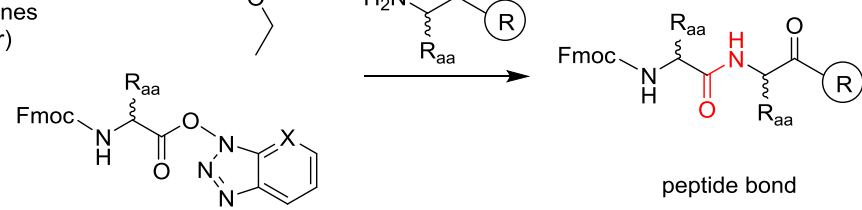

peptide bond

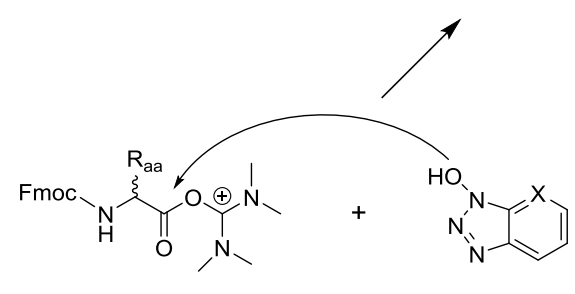

O-acylurea intermadiate $\quad \begin{aligned} & X=\mathrm{CH}, \mathrm{HOBt} \\ & \mathrm{X}=\mathrm{N}, \mathrm{HOAt}\end{aligned}$

Figure A-2 Reaction scheme of aa activation through the formation of N-hydroxylamine active esters and peptide bond formation (highlighted in red): A) Activation via DIC/Oxyma. B) Activation via benzotriazoles/base(s) (based on ref. 196 and 197). 
Solid Phase Peptide Synthesis: Acetylation (Capping)<smiles>[R17]C(N)C(=O)O</smiles>

deprotected peptide

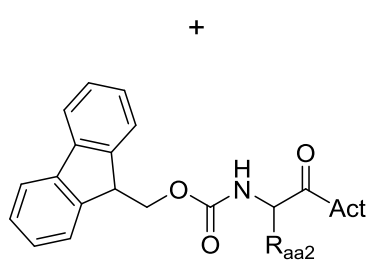

activated amino acid with Fmoc

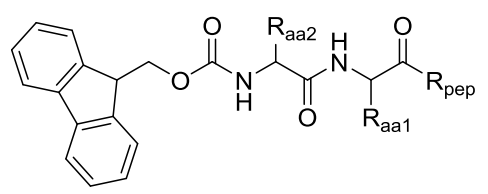

successful peptide bond formation
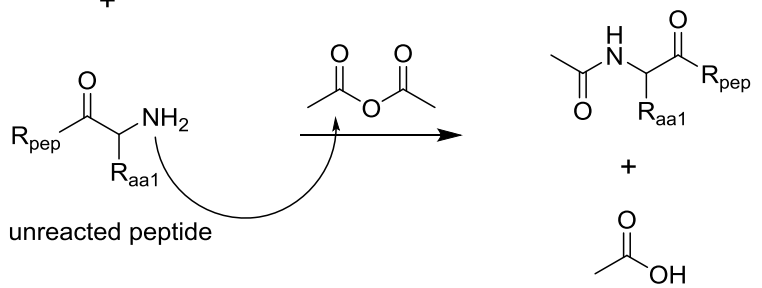

Figure A-3 Acetylation (capping) of resin bound peptides ( $\mathrm{Rpep}_{\mathrm{pe}}$ ), which have not reacted with the incoming aa building block during the coupling reaction. Acetylated peptides are excluded from further SPPS cycles ( $R_{\text {aa1 }}, R_{\text {aa2: }}$ aa residues), (based on ref. 159). 
Solid Phase Peptide Synthesis: Cleavage of the Peptide from the Resin

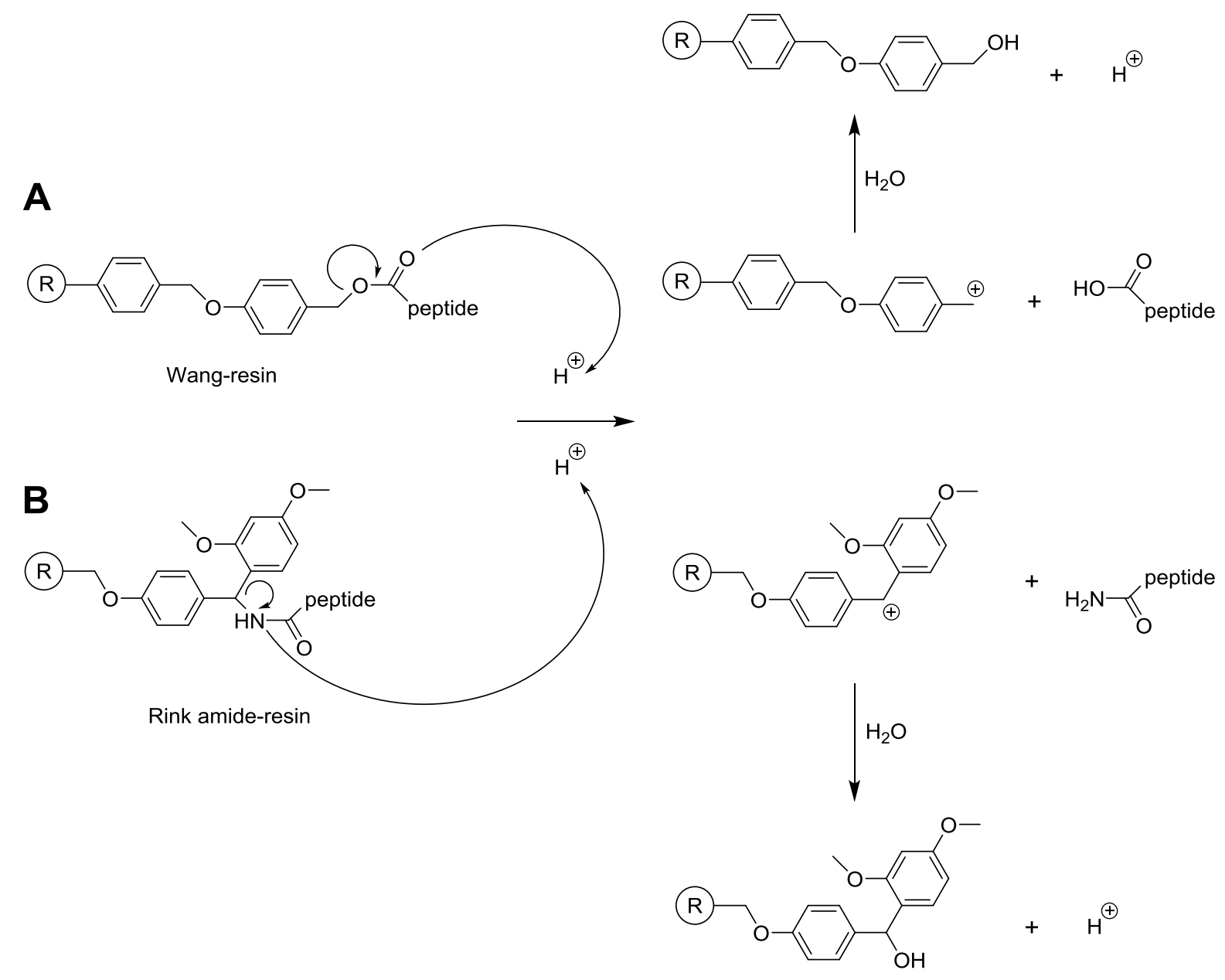

Figure A-4 After finished SPPS, the peptide must be cleaved from the solid support (resin). Depending on the applied resin, it is possible to introduce $C$-terminal $\mathbf{A}$ ) carboxylic acid groups or $\mathbf{B}$ ) amide groups. 
Solid Phase Peptide Synthesis: Scavenger Reactions

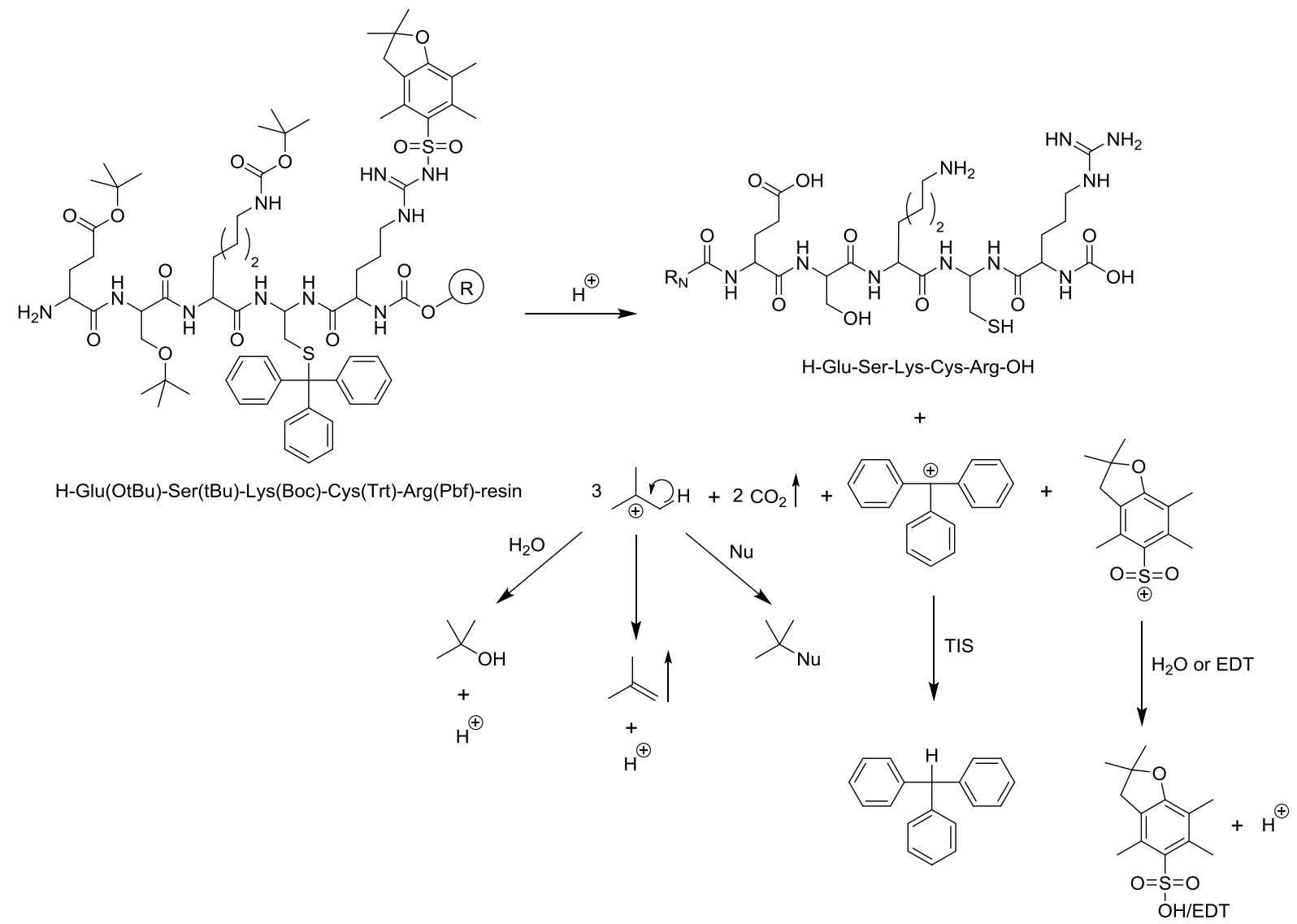

Figure A-5 Cleavage of an exemplary pentameric peptide with typical residual protecting groups ('Bu, Boc, Trt, Pbf) from the resin (R). During cleavage reactions under acidic conditions, cations emerge from the protecting groups. Thus, scavenger reagents (e.g. water, TIS and EDT) are added to TFA to transfer cations into unreactive substances. Water acts as a nucleophile, TIS hydrogenates trityl cations and EDT has reducing properties to avoid oxidation of methionine residues (not shown). Furthermore, EDT can react as a nucleophile ( $\mathrm{Nu}$ ) with carbocations (based on ref. 159, 198 and 199). 
Solid Phase Peptide Synthesis: Aspartimide Formation

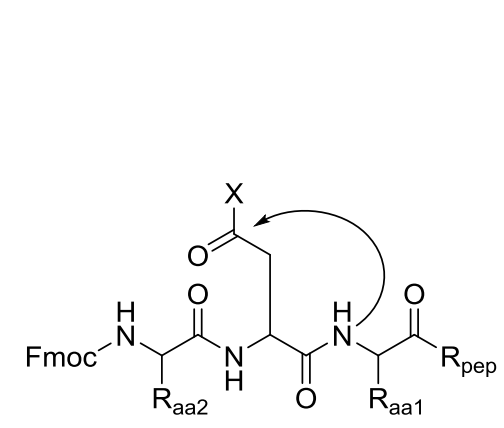<smiles>[R20]C(=O)C([R17])NC(=O)C1CC(=O)NC([R20])C1=O</smiles><smiles>[R20]C(=O)C([R17])NC(=O)C[C@@H]1NC(=O)[C@@H]([R12])NC1=O</smiles>
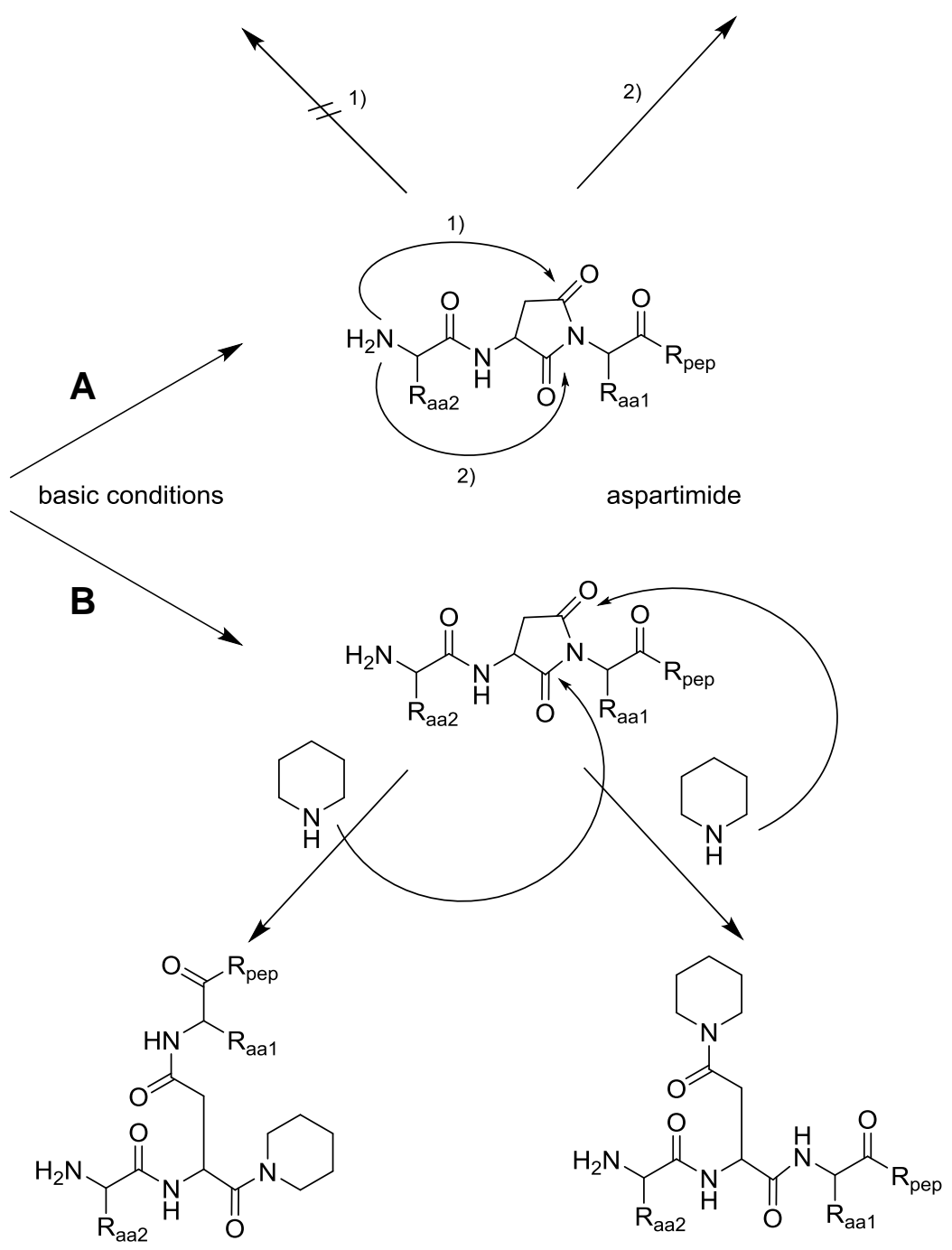

Figure A-6 Aspartimide formation under basic conditions during Fmoc-deprotection (piperidine acts as catalyst) and subsequent reactions. Aspartimide formation is facilitated when Raa1 = glycine: A) Nucleophilic attack of the $\mathrm{N}$-terminal amino group on carbonyl carbons of the aspartimide ring (sequence termination). Formation of diketopiperazine (2) is preferred over the formation of a seven-membered ring (1). B) Nucleophilic attack of the secondary amino group of piperidine on carbonyl carbons yielding piperidine-peptide adducts $\left(\mathrm{X}=\mathrm{OtBu},-\mathrm{NH}_{2} ; \mathrm{R}_{\text {pep }}=C\right.$-terminal peptide linked to solid support; $\mathrm{R}_{\mathrm{aa} 1}$, $\mathrm{R}_{\mathrm{aa} 2}=$ aa residues), (based on ref. 159, 160 and 200). 


\section{Solid Phase Peptide Synthesis: Racemisation Reactions}

A

(c)

HO-Act

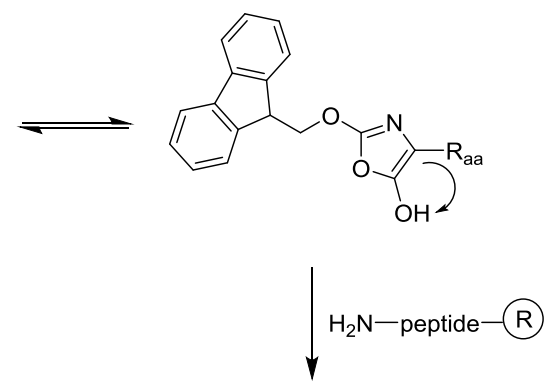

(l)

B<smiles>CCCCC(C)C(C)N(CC)C(C)C(C)C</smiles>

Figure A-7 Possible racemisation reactions during the coupling reaction: A) Racemisation via the formation of oxazolone derivatives of $C$-terminal activated aa. However, oxazolone formation is unlikely concerning a single aa. It is more probable, if peptide fragments are $C$-terminal activated. B) Racemisation of a cysteine building blocks during the activation reaction, if bases (e.g. DIPEA) are used which deprotonate the $\alpha$-carbon. A similar reaction is conceivable during the deprotection with piperidine. Elevated temperatures facilitate deprotonation as well (based on ref. 159 and 201). 


\section{Comparison of Native and Artificial Sequences}

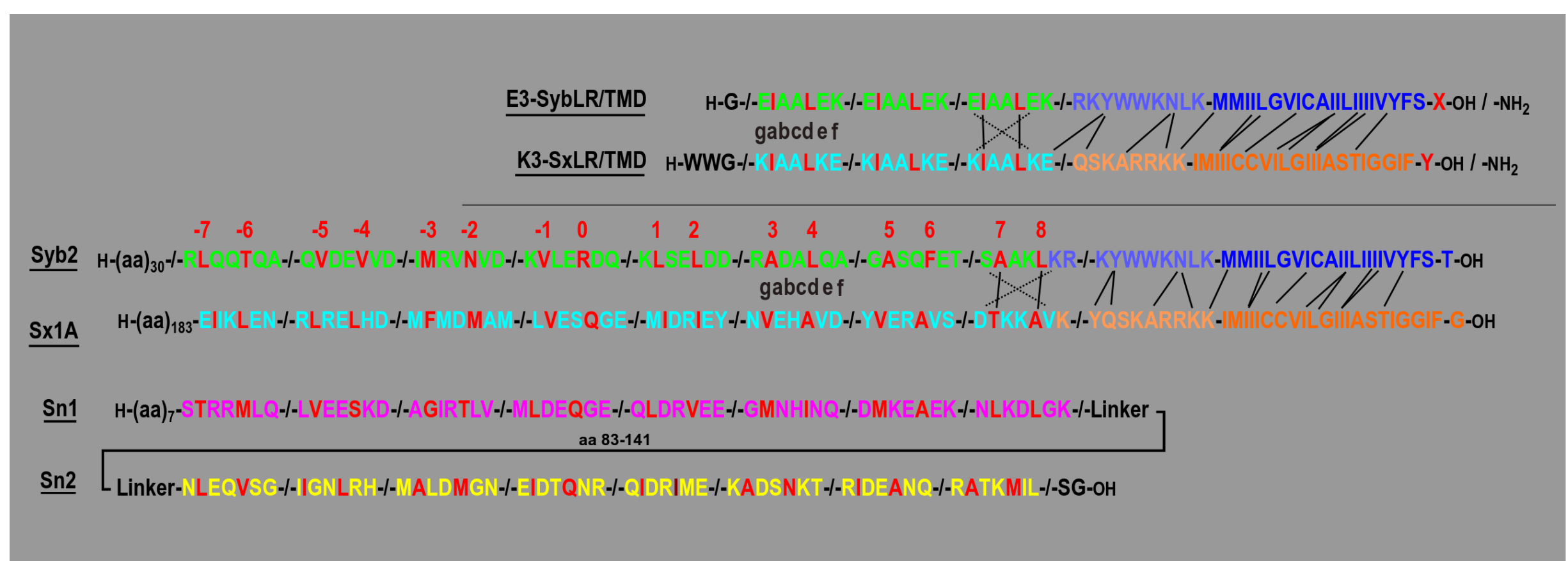

Figure A-8 Comparison of sequences of the SNARE core complex components (Syb2, Sx1A, SNAP 25 (Sn1 and Sn2)) with sequences of the SNARE mimetics (E3-SybLR/TMD and K3-SxLR/TMD): The 16 in plane interacting aas $(-7-8)$ of the four SNARE core complex components are given in red, respectively. The corresponding aas of the E3 and K3 recognition units are also highlighted in red. The hydrophobic and hydrophilic interactions, which are typical for coiled coils, are highlighted by solid and dashed lines, respectively. Possible interactions due to spatial proximity within the LRs/TMDs are also given as solid lines (based on ref. 10, 16 and 127). 


\section{Chromatography Results of PNA-LR/TMD Peptide Hybrids}

Table A-1 Overview of analytical UPLC runs: Purities of PNA-LR/TMD peptide hybrids were determined by the integration of chromatograms.

\begin{tabular}{|l|c|c|c|c|c|}
\hline \multicolumn{1}{|c|}{ sample } & $\begin{array}{c}\text { purity } \\
{[\%]}\end{array}$ & $\begin{array}{c}\text { area } \\
\text { [a.u.] }\end{array}$ & $\begin{array}{c}\text { time range } \\
\text { [min] }\end{array}$ & $\begin{array}{c}\text { areal } \\
\text { [a.u.] }\end{array}$ & $\begin{array}{c}\text { time range } \\
\text { [min] }\end{array}$ \\
\hline PNA1/SybT & 24.1 & 114.1 & $1.5-14.1$ & 27.5 & $10.7-11.3$ \\
\hline PNA2/SxG & 14.6 & 1061.0 & $1.5-18.6$ & 154.6 & $9.6-10.3$ \\
\hline PNA3/SxG & 24.8 & 93.6 & $1.5-12.8$ & 23.2 & $9.8-10.4$ \\
\hline PNA1/SybE & 16.8 & 411.2 & $1.5-14.7$ & 69.2 & $8.9-9.5$ \\
\hline PNA2/SxE & 21.9 & 1322.0 & $1.5-18.5$ & 288.9 & $8.9-9.5$ \\
\hline PNA3/SxE & 34.4 & 329.4 & $1.5-17.0$ & 113.4 & $9.1-9.7$ \\
\hline PNA1/SybK & 21.2 & 293.0 & $1.5-14.8$ & 62.0 & $7.8-8.2$ \\
\hline PNA1/SxK & 14.0 & 1429.0 & $1.5-19.0$ & 200.2 & $8.6-9.2$ \\
\hline PNA2/SxK & 14.3 & 605.7 & $1.5-18.4$ & 86.8 & $8.7-9.2$ \\
\hline PNA3/SxK & 16.7 & 119.5 & $1.5-16.0$ & 20.0 & $8.8-9.2$ \\
\hline PNA1/SybTa & 32.3 & 510.4 & $1.5-13.4$ & 164.7 & $8.7-9.3$ \\
\hline PNA2/SxGa & 28.5 & 302.3 & $1.5-17.6$ & 86.3 & $10.0-10.7$ \\
\hline PNA3/SxGa & 14.8 & 986.9 & $1.5-18.4$ & 145.9 & $10.3-11.0$ \\
\hline PNA1/SybEa & 38.0 & 71.2 & $1.5-13.2$ & 27.0 & $9.6-10.0$ \\
\hline PNA2/SxEa & 24.7 & 256.3 & $1.5-17.3$ & 63.4 & $9.6-10.3$ \\
\hline PNA3/SxEa & 29.2 & 205.8 & $1.5-16.4$ & 60.0 & $9.5-10.2$ \\
\hline PNA1/SybKa & 26.1 & 598.0 & $1.5-15.0$ & 156.2 & $7.8-8.6$ \\
PNA2/SxKa & 22.4 & 375.7 & $1.5-18.3$ & 84.1 & $8.9-9.5$ \\
\hline PNA3/SxKa & 33.0 & 64.8 & $1.5-15.1$ & 21.4 & $8.9-9.5$ \\
\hline Remars:mp: & & & & \\
\hline
\end{tabular}

Remarks: mp: main peak. 


\section{$\underline{\text { Appendix }}$}

PNA1/SybT

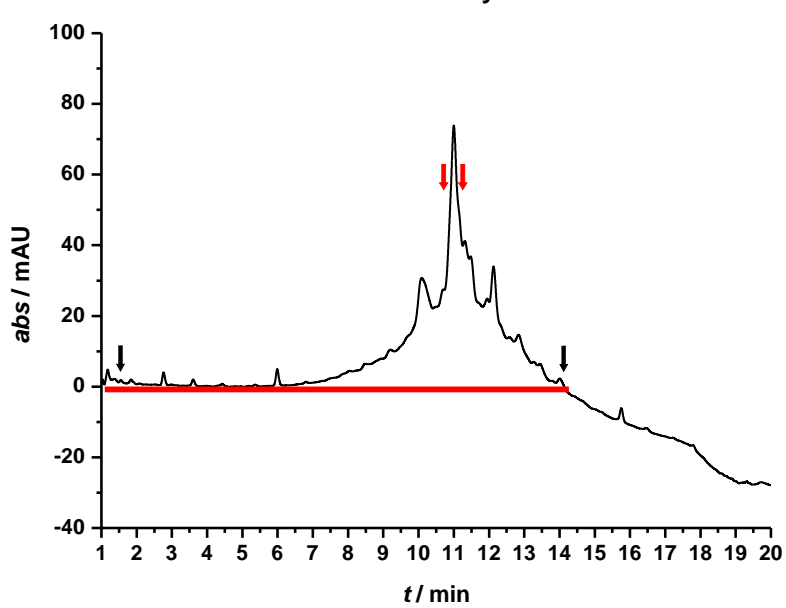

PNA1/SybK

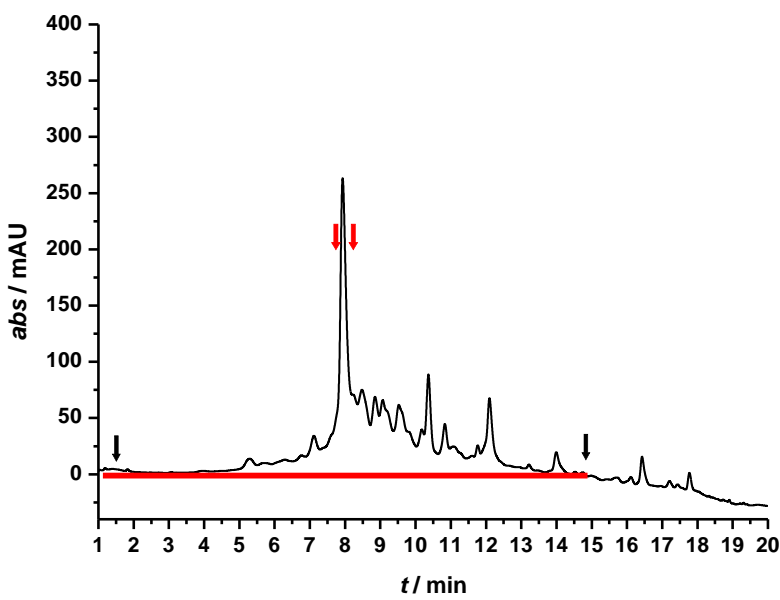

PNA1/SybEa

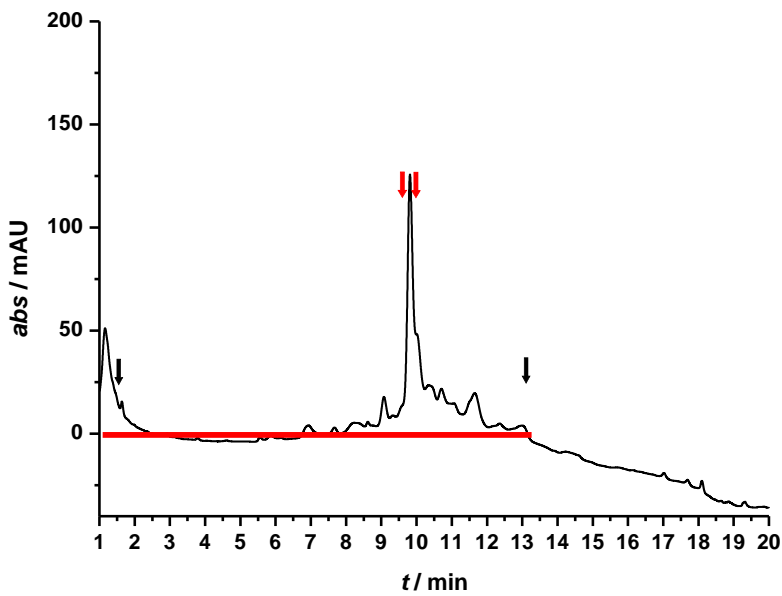

PNA1/SybE

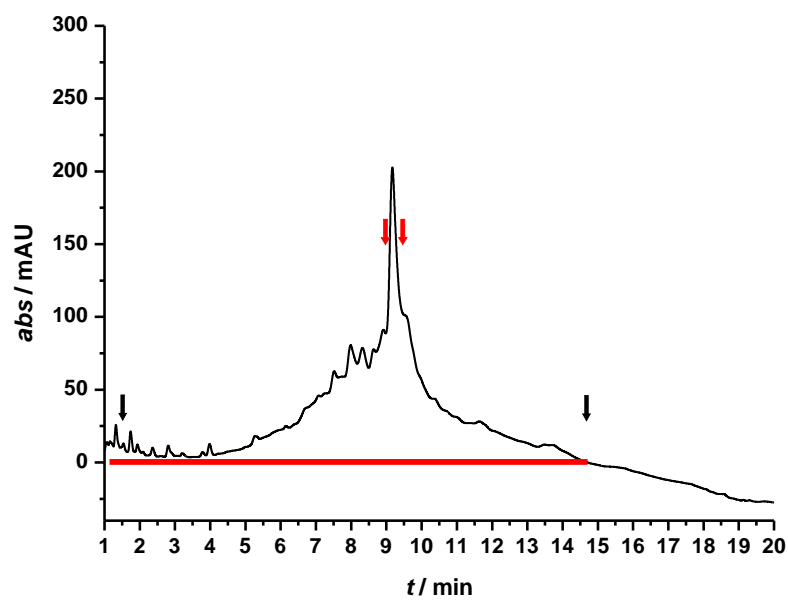

PNA1/SybTa

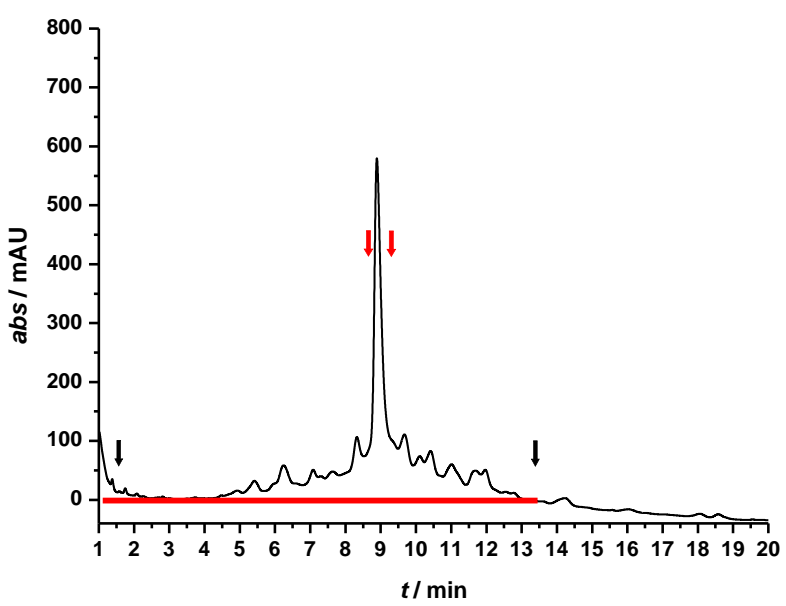

PNA1/SybKa

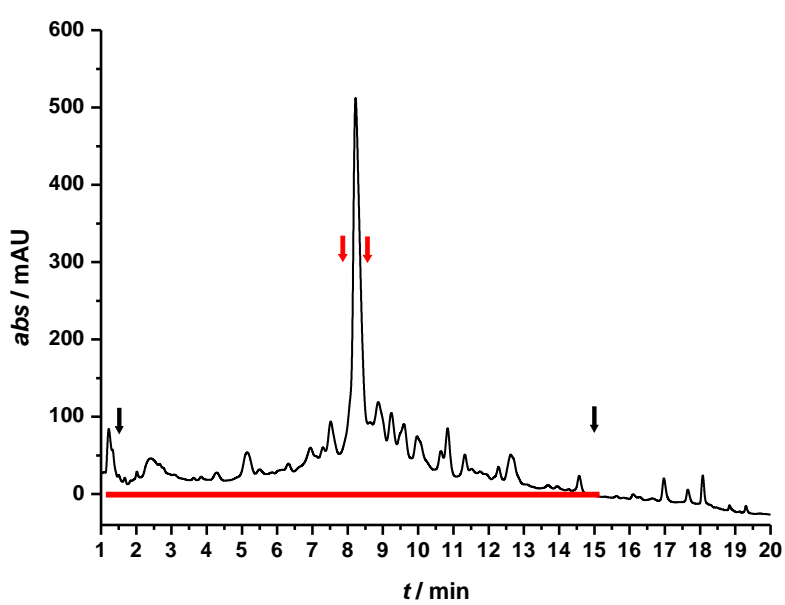

Figure A-9 Chromatograms of PNA1/SybX(a) peptides measured by analytical UPLC. The detection wavelength was $215 \mathrm{~nm}$. Black arrows indicate the total integrated area and red arrows the integrated area of the main peak (raw data were edited by using the OriginPro 8.5G software). 


\section{$\underline{\text { Appendix }}$}

PNA2/SxG

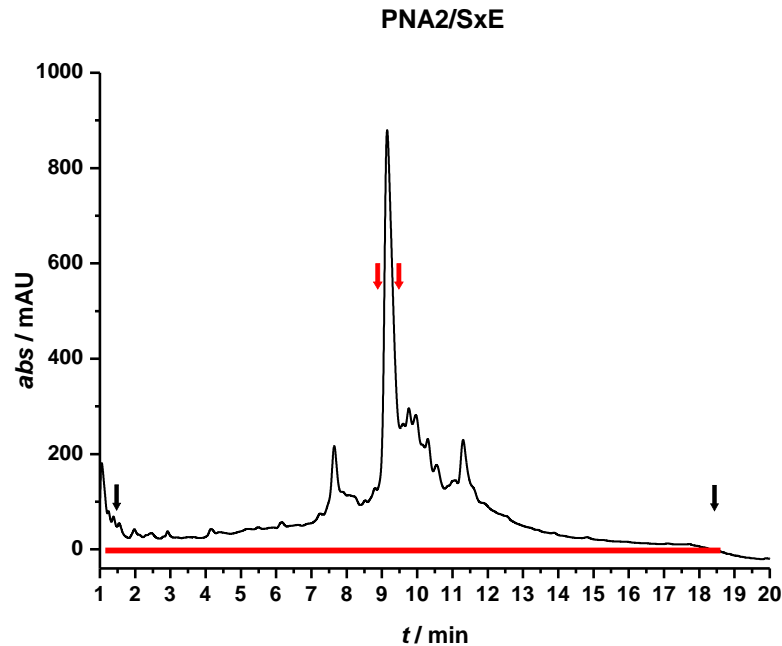

PNA2/SxK
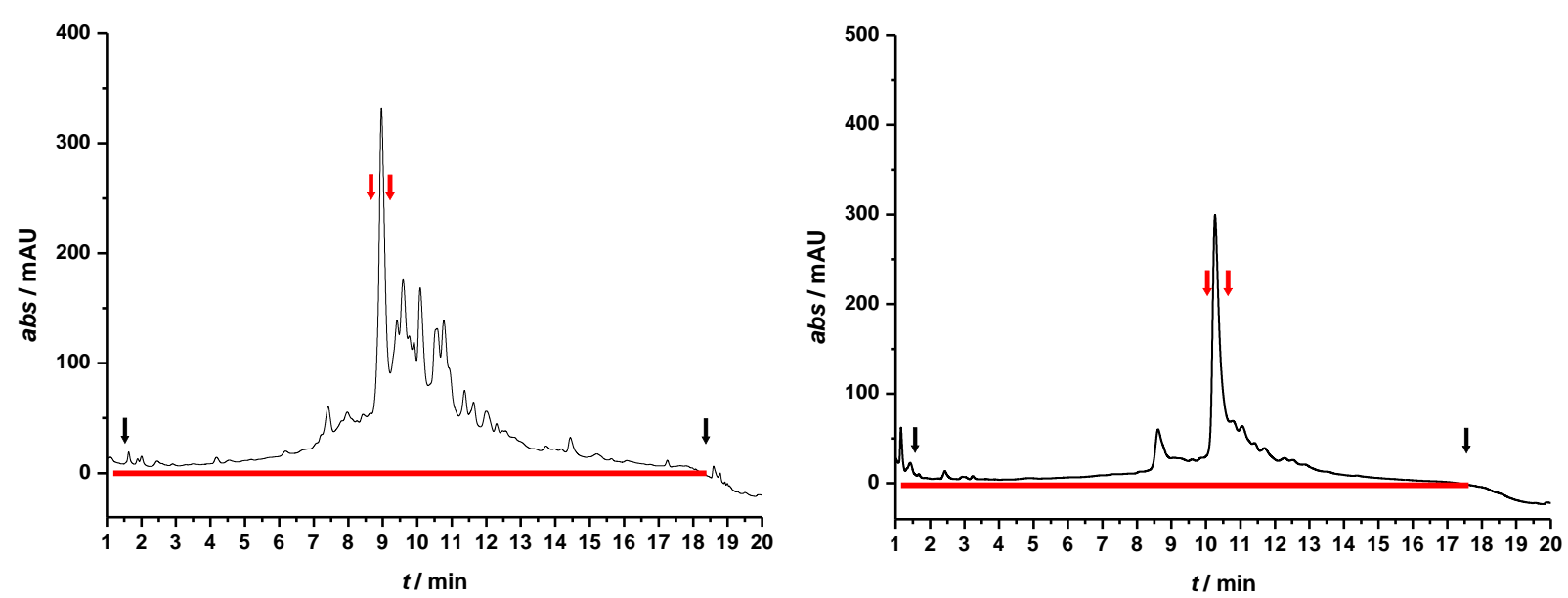

PNA2/SxEa

PNA2/SxKa
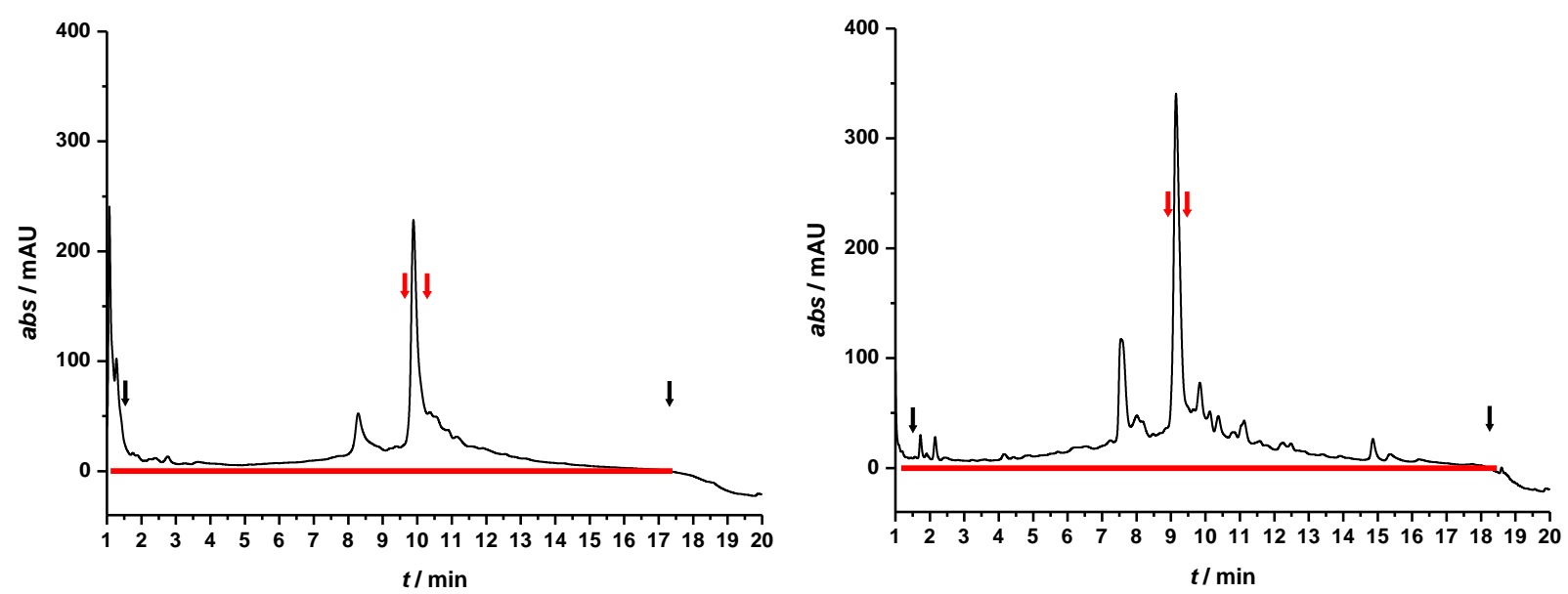

Figure A-10 Chromatograms of PNA2/SxY(a) peptides measured by analytical UPLC. The detection wavelength was $215 \mathrm{~nm}$. Black arrows indicate the total integrated area and red arrows the integrated area of the main peak (raw data were edited by using the OriginPro 8.5G software). 


\section{$\underline{\text { Appendix }}$}

PNA3/SxG

PNA3/SXE
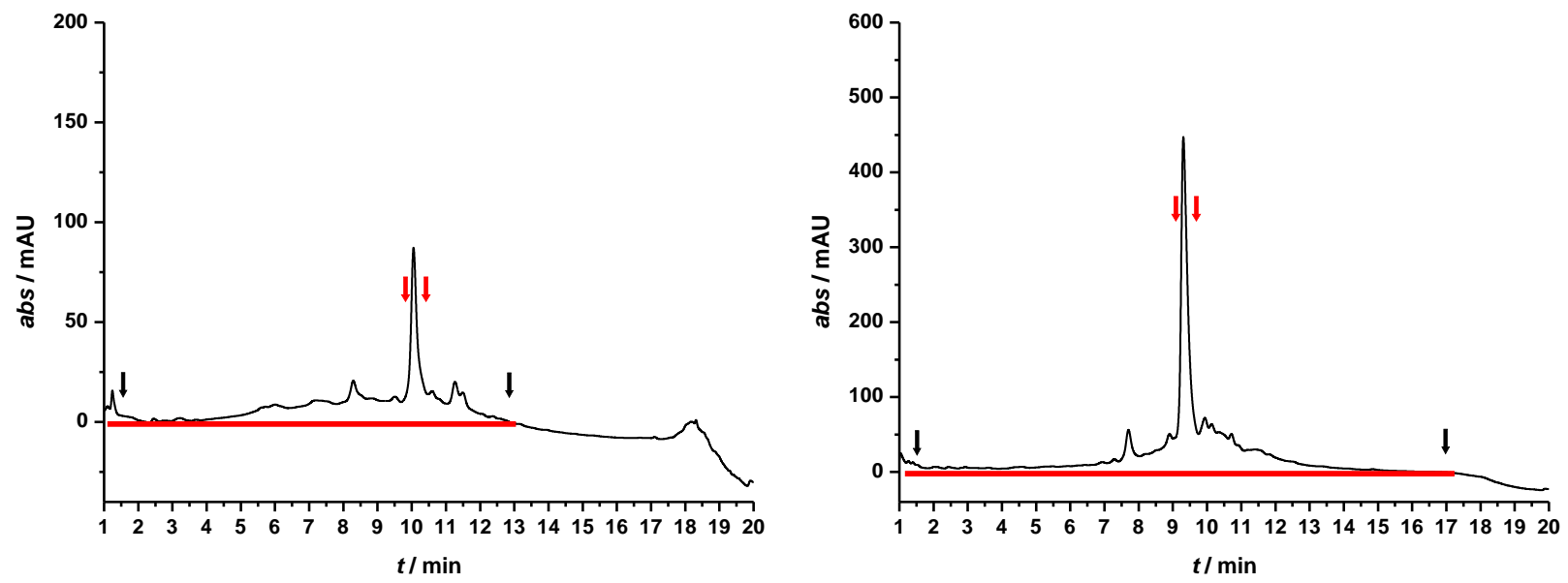

PNA3/SXK
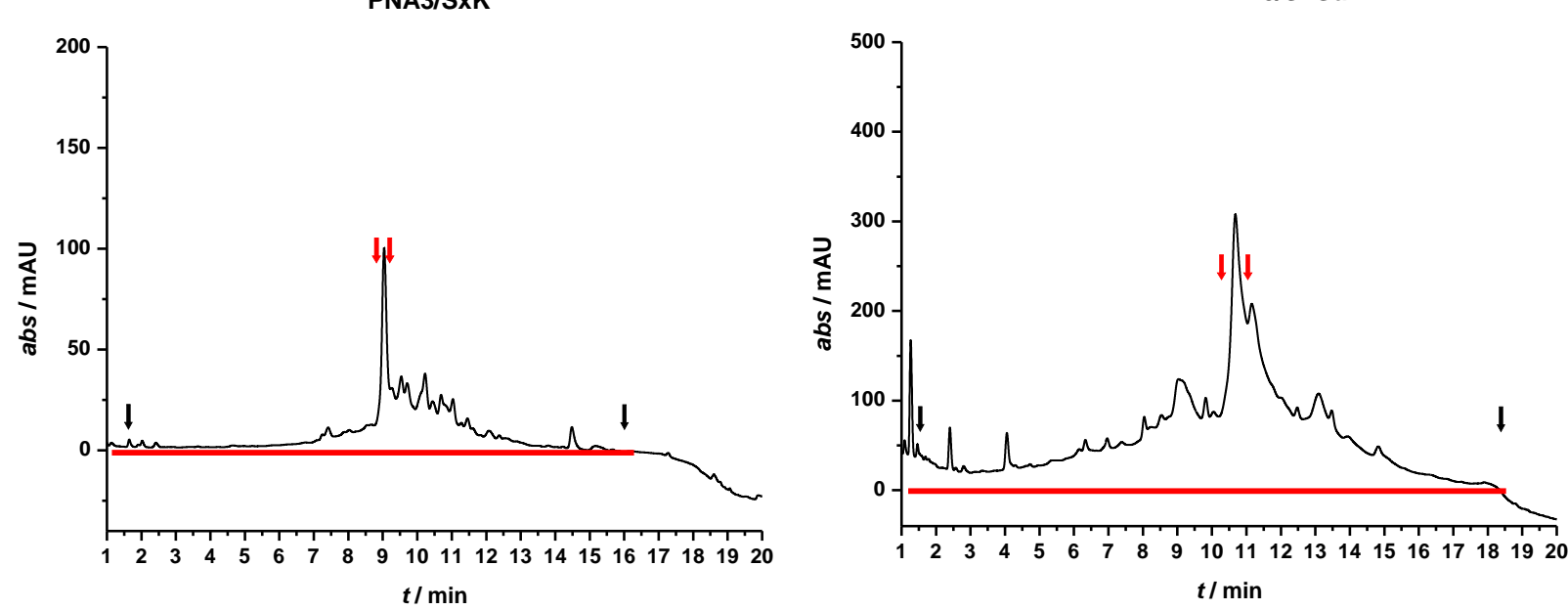

PNA3/SxEa

PNA3/SxKa
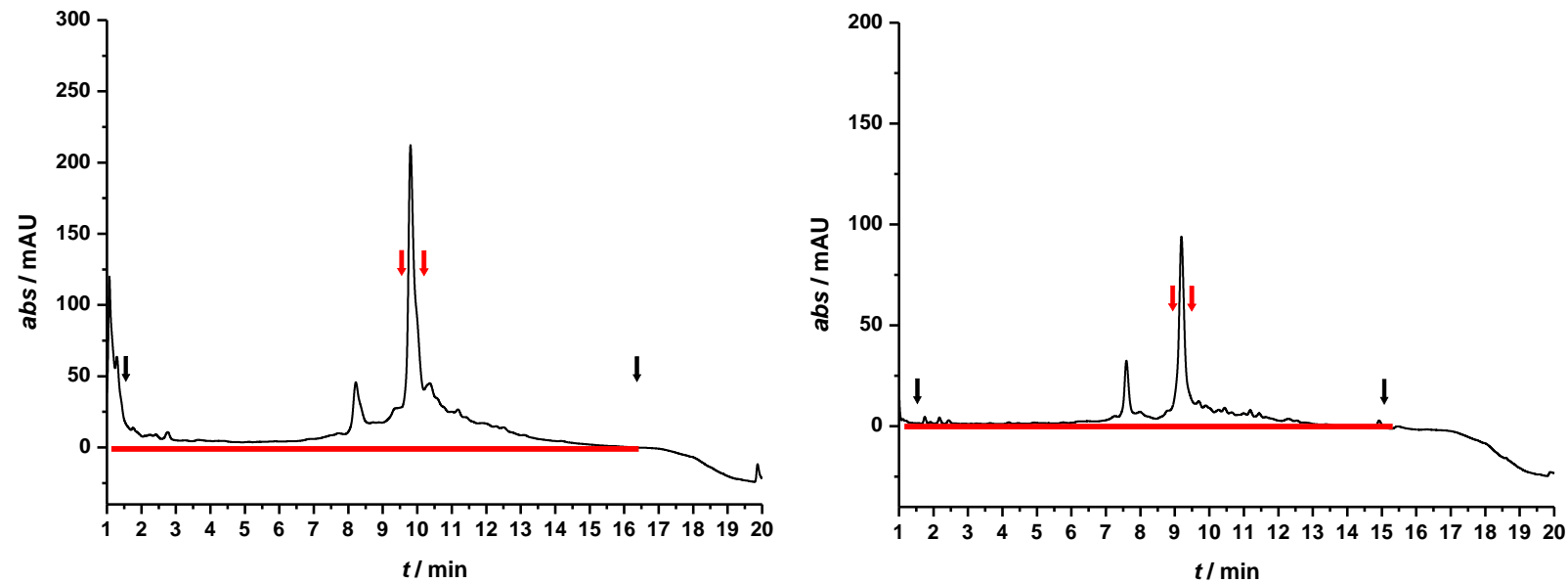

Figure A-11 Chromatograms of PNA3/SxY(a) peptides measured by analytical UPLC. The detection wavelength was $215 \mathrm{~nm}$. Black arrows indicate the total integrated area and red arrows the integrated area of the main peak (raw data were edited by using the OriginPro $8.5 \mathrm{G}$ software). 


\section{$\underline{\text { Appendix }}$}

PNA1/SxK

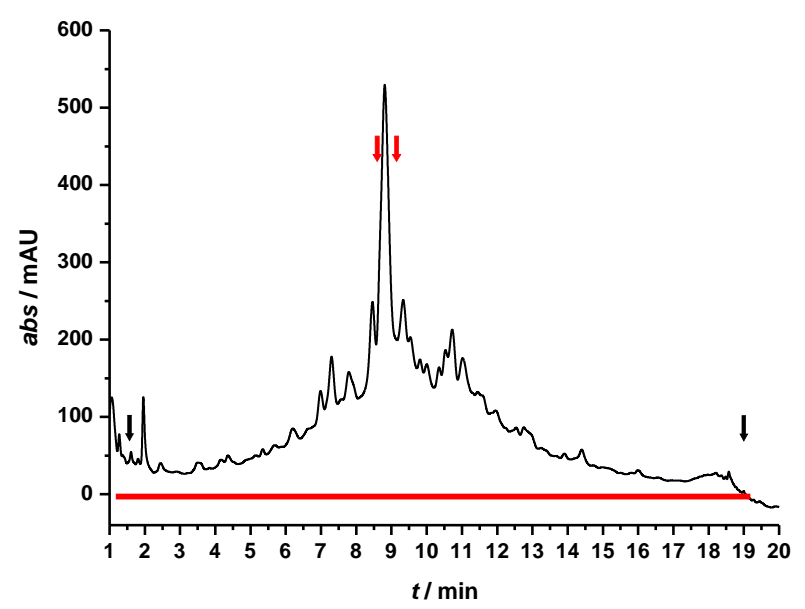

Figure A-12 Chromatogram of the PNA1/SxK peptide measured by analytical UPLC. The detection wavelength was $215 \mathrm{~nm}$. Black arrows indicate the total integrated area and red arrows the integrated area of the main peak (raw data were edited by using the OriginPro $8.5 \mathrm{G}$ software). 


\section{$\underline{\text { Appendix }}$}

\section{Chromatography Results of E3/K3-LR/TMD Peptide Hybrids}

Table A-2 Overview of analytical UPLC runs: Purities of (i)E3/K3-LR/TMD peptide hybrids were determined by integration of the chromatograms. E3/SybK $\mathrm{H}_{\text {PLC }}$ and $\mathrm{K} 3 / \mathrm{S} \times \mathrm{E}_{\text {HPLC }}$ samples were purified by semi-preparative HPLC.

\begin{tabular}{|l|c|c|c|c|c|}
\hline \multicolumn{1}{|c|}{ sample } & $\begin{array}{c}\text { purity } \\
\text { [\%] }\end{array}$ & $\begin{array}{c}\text { area }_{\text {total }} \\
\text { [a.u.] }\end{array}$ & $\begin{array}{c}\text { time range } \\
\text { [min] }\end{array}$ & $\begin{array}{c}\text { area }_{\text {mp }} \\
\text { [a.u.] }\end{array}$ & $\begin{array}{c}\text { time range } \\
\text { [min] }\end{array}$ \\
\hline E3/SybT & 40.5 & 402.0 & $1.5-15.1$ & 163.0 & $9.5-10.4$ \\
\hline iE3/SybT & 18.8 & 1289.0 & $1.5-19.7$ & 242.3 & $12.3-13.7$ \\
\hline K3/SxG & 28.0 & 764.7 & $1.5-17.2$ & 213.8 & $10.4-11.4$ \\
\hline E3/SybE & 42.9 & 507.8 & $1.5-13.7$ & 217.9 & $7.0-8.0$ \\
\hline K3/SxE & 30.9 & 1327.0 & $1.5-18.9$ & 410.3 & $9.7-10.6$ \\
K3/SxE & 44.8 & 949.8 & $1.5-19.1$ & 425.1 & $9.6-10.5$ \\
\hline E3/SybK & 18.3 & 1209.0 & $1.5-18.7$ & 221.0 & $9.9-10.5$ \\
\hline E3/SybKHPLC & 61.2 & 126.9 & $1.5-15.1$ & 77.6 & $12.2-12.7$ \\
\hline K3/SxK & 22.1 & 2429.0 & $1.5-19.5$ & 535.6 & $8.8-9.7$ \\
\hline E3/SybTa & 27.9 & 204.0 & $1.5-17.2$ & 57.0 & $10,8-11.7$ \\
\hline K3/SxGa & 25.6 & 294.7 & $1.5-18.6$ & 59.8 & $11.1-11.7$ \\
\hline E3/SybEa & 29.8 & 568.4 & $1.5-14.6$ & 169.6 & $7.9-8.9$ \\
\hline K3/SxEa & 25.6 & 810.9 & $1.5-18.6$ & 207.7 & $10.2-11.0$ \\
\hline E3/SybKa & 32.0 & 354.8 & $1.5-14.2$ & 113.4 & $10.5-11.1$ \\
\hline K3/SxKa & 20.9 & 229.2 & $1.5-19.0$ & 47.8 & $9.8-10.3$ \\
\hline RemaKS: & & & & \\
\hline
\end{tabular}

Remarks: mp: main peak. 


\section{Appendix}

E3/SybT

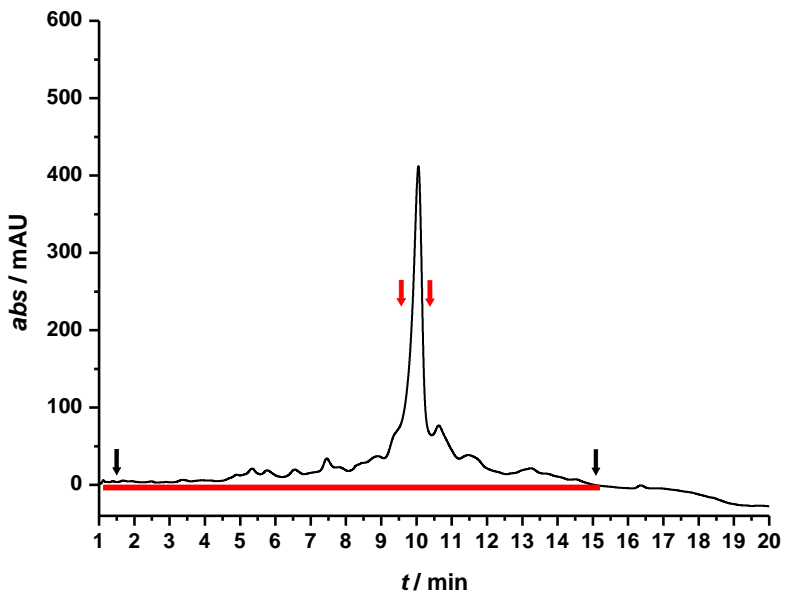

E3/SybK

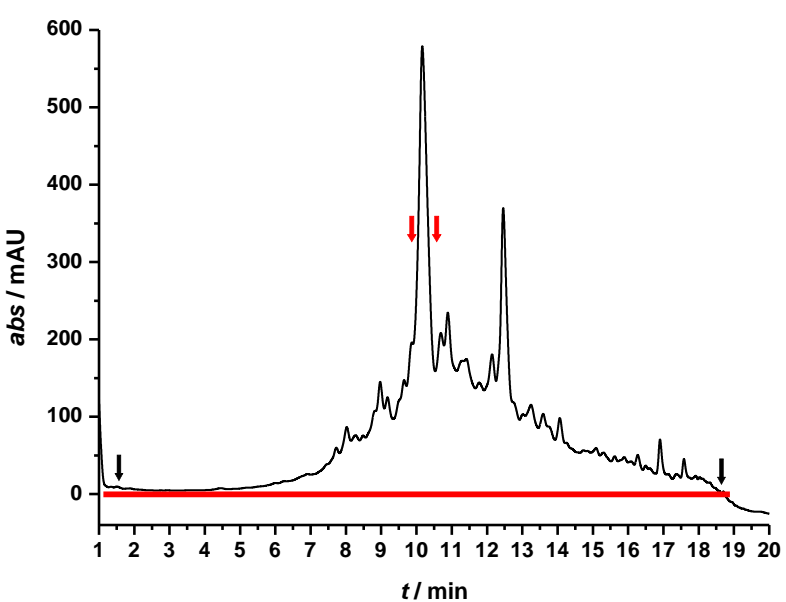

E3/SybE

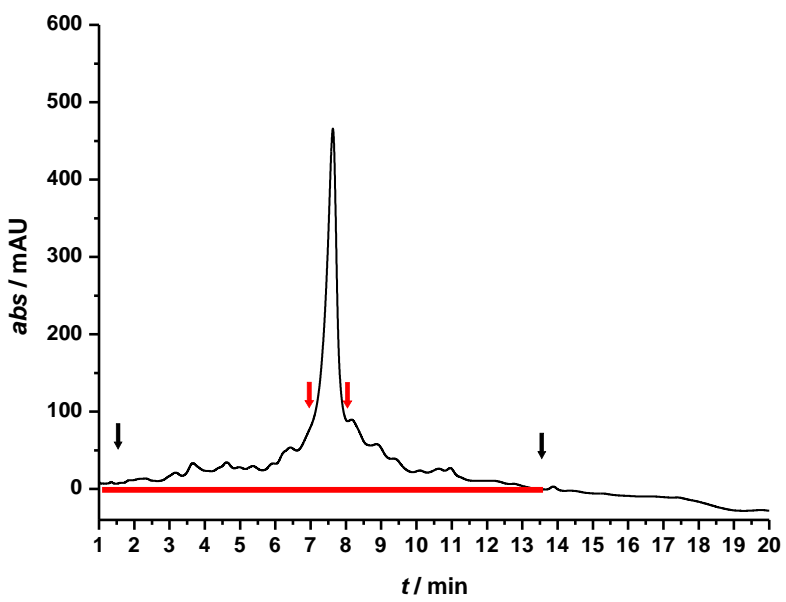

iE3/SybT

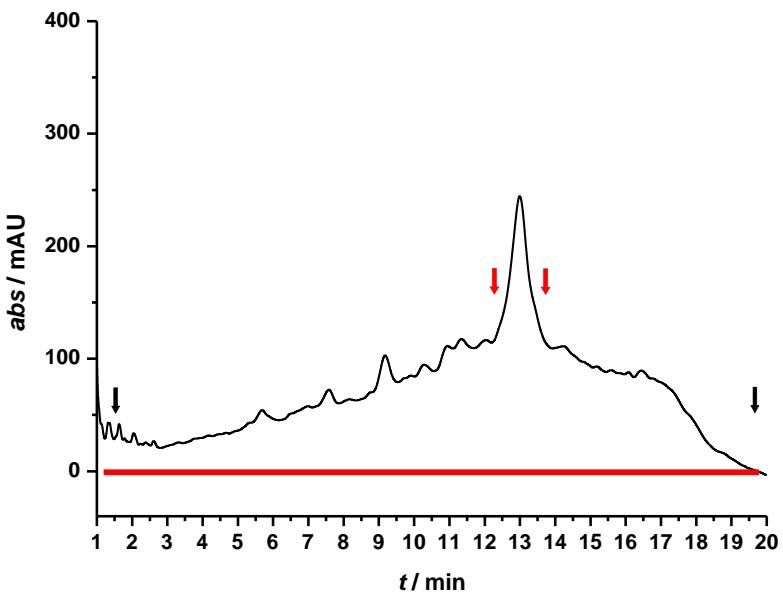

E3/SybK $K_{\text {HPLC }}$

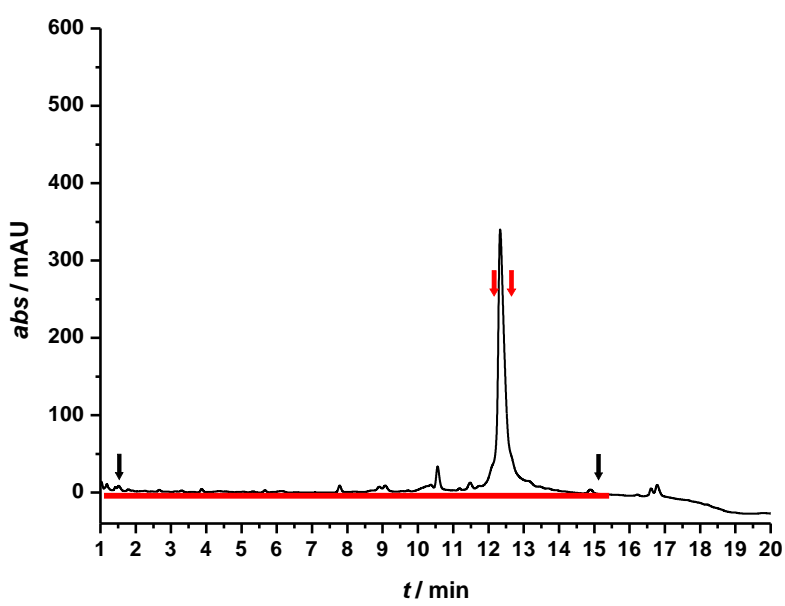

E3/SybTa

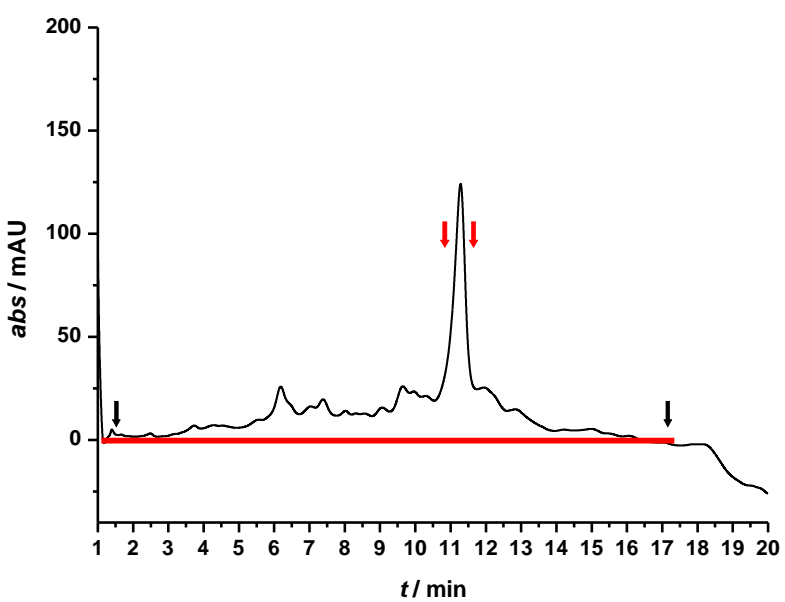




\section{$\underline{\text { Appendix }}$}

E3/SybEa

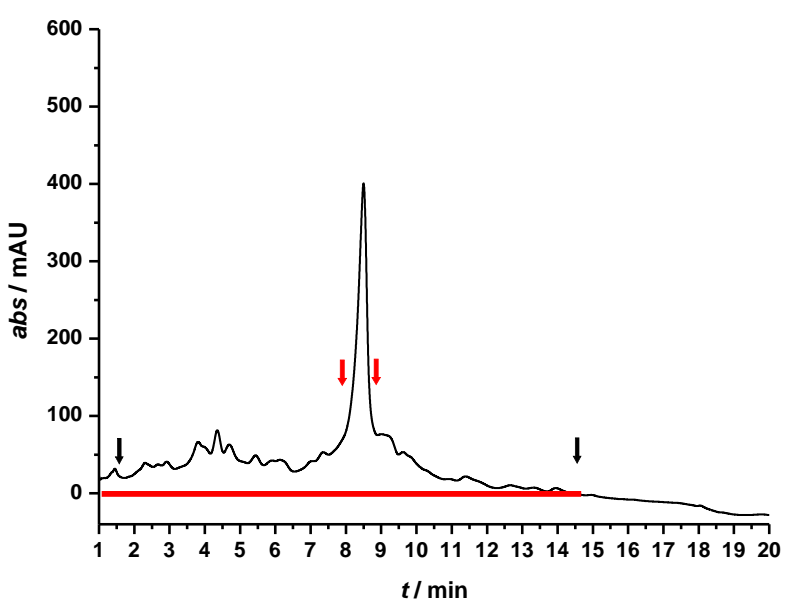

E3/SybKa

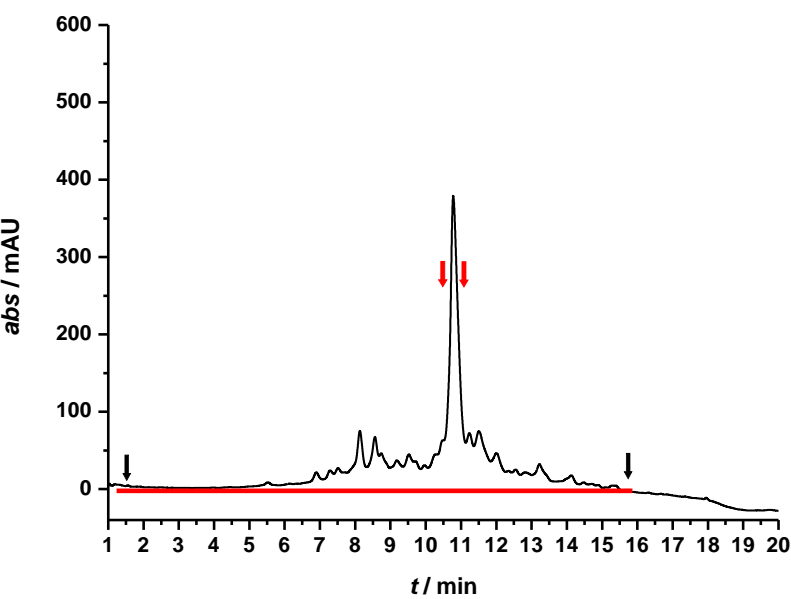

Figure A-13 Chromatograms of (i)E3/SybX(a) peptides measured by analytical UPLC. The detection wavelength was $215 \mathrm{~nm}$. Black arrows indicate the total integrated area and red arrows the integrated area of the main peak (raw data were edited by using the OriginPro $8.5 \mathrm{G}$ software). 


\section{Appendix}

K3/SxG

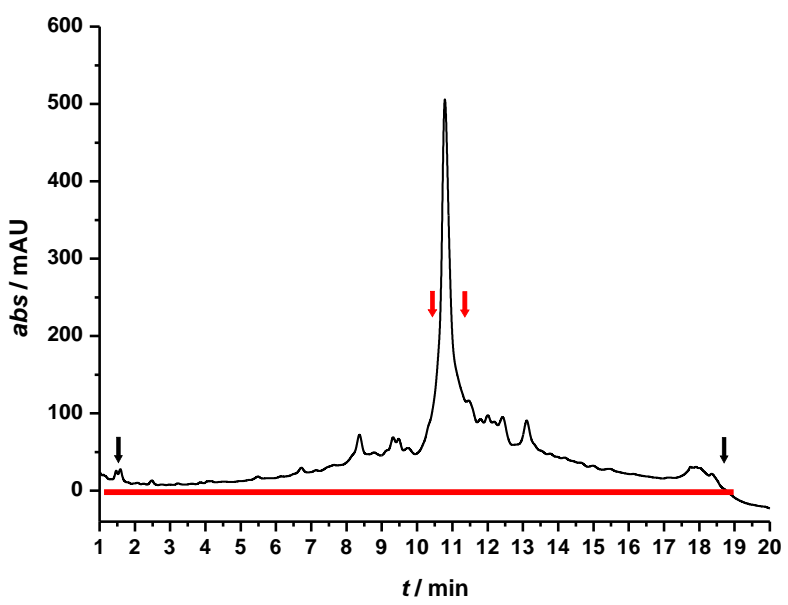

$\mathrm{K} 3 / \mathrm{SxE}_{\mathrm{HPLC}}$

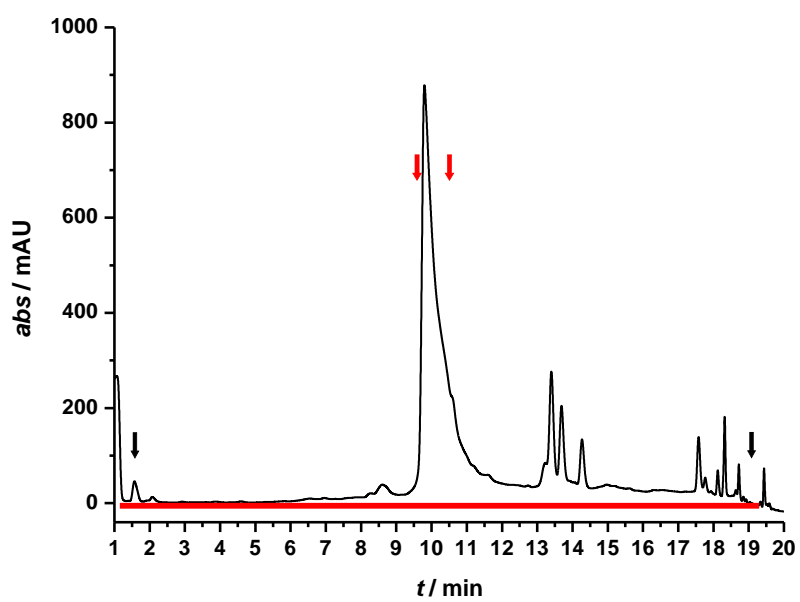

K3/SxGa

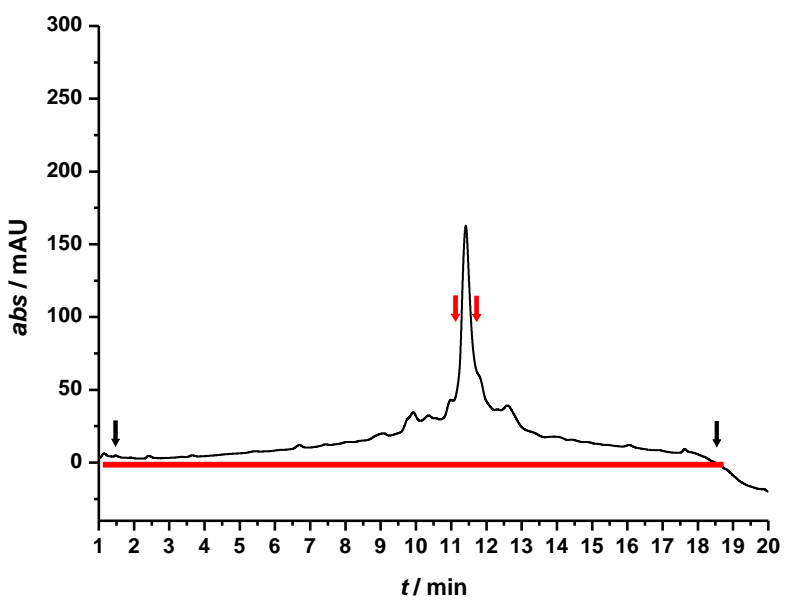

K3/SxE

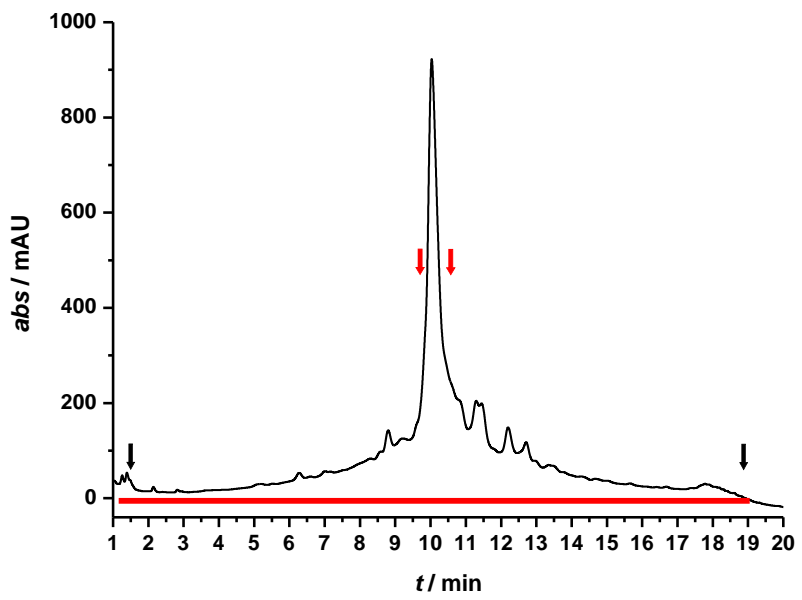

K3/SxK

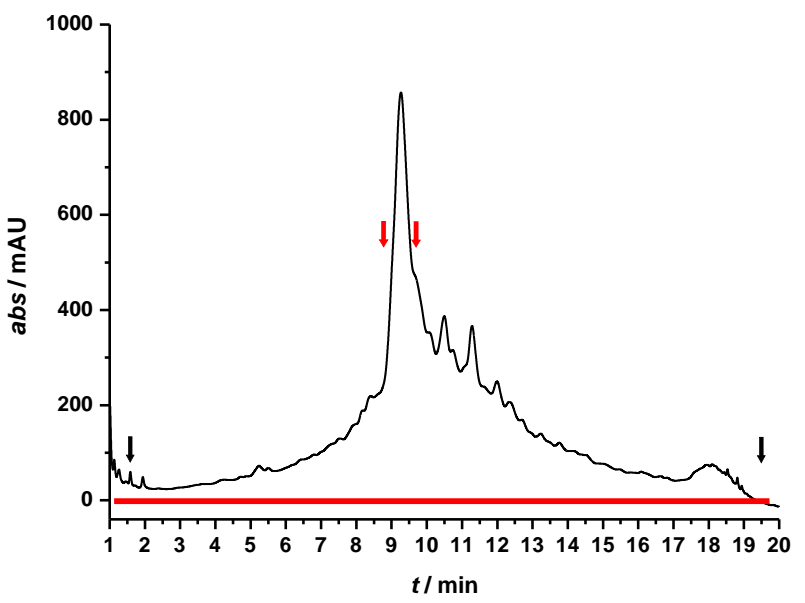

K3/SxEa

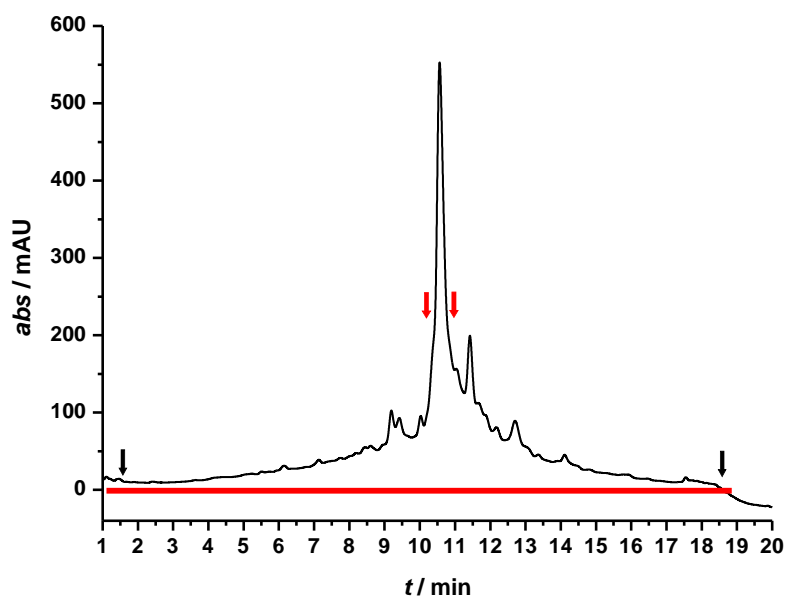




\section{$\underline{\text { Appendix }}$}

K3/SxKa

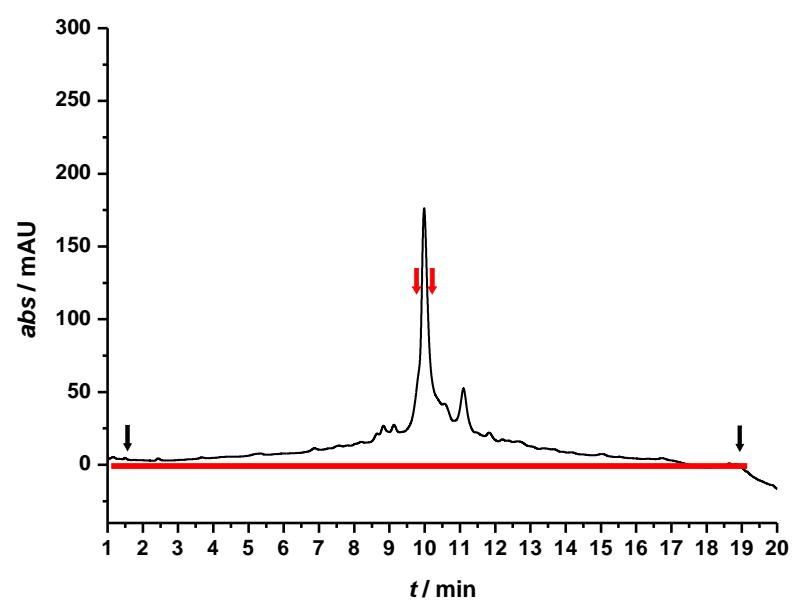

Figure A-14 Chromatograms of K3/SxY(a) peptides measured by analytical UPLC. The detection wavelength was $215 \mathrm{~nm}$. Black arrows indicate the total integrated area and red arrows the integrated area of the main peak (raw data were edited by using the OriginPro $8.5 \mathrm{G}$ software). 


\section{Loss of Lipid Material During Proteoliposome Preparation and Size Exclusion Chromatography}

Table A-3 Comparison of the lipid loss of liposomes decorated with PNA-LR/TMD peptide hybrids. Losses were determined by means of phosphate tests.

\begin{tabular}{|c|c|c|c|c|}
\hline sample & $\begin{array}{c}m(P) \text { after extr. } \\
{[\mu \mathrm{g}]}\end{array}$ & $\begin{array}{c}\text { Ioss (extr.) } \\
{[\%]^{\mathrm{a}}}\end{array}$ & $\begin{array}{c}\text { loss (size ex.) } \\
{[\%]^{b}}\end{array}$ & lipid ratioc \\
\hline PNA1/SybT & 0.78 & 33 & 78 & - \\
\hline PNA2/SxG & 0.79 & 32 & - & 1.0 to 4.0 \\
\hline PNA3/SxG & 0.84 & 28 & - & 1.0 to 4.3 \\
\hline PNA1/SybE & 0.77 & 34 & 55 & - \\
\hline PNA2/SxE & 0.84 & 28 & - & 1.0 to 4.4 \\
\hline PNA3/SxE & 0.83 & 28 & - & 1.0 to 4.3 \\
\hline PNA1/SybK & 0.93 & 20 & 61 & - \\
\hline PNA1/SxK & 0.79 & 32 & - & 1.0 to 3.4 \\
\hline PNA2/SxK & 0.98 & 16 & - & 1.0 to 4.2 \\
\hline PNA3/SxK & 0.74 & 36 & - & 1.0 to 3.2 \\
\hline PNA1/SybTa & 0.74 & $37^{d}$ & 74 & - \\
\hline PNA2/SxGa & 0.75 & 36 & - & 1.0 to 4.1 \\
\hline PNA3/SxGa & 0.79 & 32 & - & 1.0 to 4.3 \\
\hline PNA1/SybEa & 0.67 & 42 & 43 & - \\
\hline PNA2/SxEa & 0.76 & 35 & - & 1.0 to 3.6 \\
\hline PNA3/SxEa & 0.89 & 23 & - & 1.0 to 3.0 \\
\hline PNA1/SybKa & 0.79 & $32^{\mathrm{e}}$ & 85 & - \\
\hline PNA2/SxKa & 0.67 & 42 & - & 1.0 to 3.4 \\
\hline PNA3/SxKa & 0.84 & 28 & - & 1.0 to 4.3 \\
\hline
\end{tabular}

Remarks: a-b) Loss of lipids: a) calculated by using the supposed mass of phosphorus before extrusion $(1.16 \mu \mathrm{g})$ and measured masses of phosphorus after extrusion ( $2^{\text {nd }}$ column). b) calculated by using measured masses of phosphorus before and after size exclusion chromatography (values are not shown). c) Actual lipid ratios between labelled (PNA1/SybX(a)) and unlabelled (PNA/SxY(a)) proteoliposomes during leaflet mixing assays deviates from the supposed ratio of 1 to 4 . d-e) Loss of lipids calculated by using measured masses of phosphorus before extrusion (value is not shown) and after extrusion ( $2^{\text {nd }}$ column): d) $31 \%$ and e) $41 \%$. Abbreviations: extr.: extrusion, size ex.: size exclusion chromatography. 


\section{$\underline{\text { Appendix }}$}

Table A-4 Loss of lipids during proteoliposome preparation determined by means of phosphate tests and Rh emission.

\begin{tabular}{|l|c|c|}
\hline \multicolumn{1}{|c|}{ sample } & $\begin{array}{c}\text { loss determined by } \mathrm{PO}_{4} \text { test } \\
{[\%]^{\mathrm{a}}}\end{array}$ & $\begin{array}{c}\text { loss determined by Rh em. } \\
{[\%]^{\mathrm{c}}}\end{array}$ \\
\hline PNA1/SybK & 41 & 31 \\
\hline PNA1/Syba & 31 & 42 \\
\hline PNA1/SybKa & 32 & 46 \\
\hline
\end{tabular}

Remarks: a-b) Loss of lipids: a) calculated by using the supposed mass of phosphorus before extrusion $(1.16 \mu \mathrm{g})$ and masses of phosphorus after extrusion. b) calculated by using the measured masses of phosphorus before and after extrusion. c) Loss of lipids was calculated by the decrease of emission intensity at $585 \mathrm{~nm}$ of Rh-DOPE before and after extrusion. 


\section{$\underline{\text { Appendix }}$}

Table A-5 Comparison of lipid loss of liposomes decorated with E3/K3-LR/TMD peptide hybrids determined by means of phosphate tests.

\begin{tabular}{|c|c|c|c|c|}
\hline sample & $\begin{array}{c}m(P) \text { after extr. } \\
{[\mu \mathrm{g}]}\end{array}$ & $\begin{array}{c}\text { loss (extr.) } \\
{[\%]^{\mathrm{a}}}\end{array}$ & $\begin{array}{c}\text { loss (size ex.) } \\
{[\%]^{\mathrm{b}}}\end{array}$ & lipid ratioc \\
\hline E3/SybT & 0.83 & 28 & 48 & - \\
\hline K3/SxG & 0.58 & 50 & - & 1.0 to 2.8 \\
\hline Liposomes & 1.10 & 6 & - & 1.0 to 5.3 \\
\hline iE3/SybT & 0.81 & 30 & 58 & - \\
\hline K3/SxG & 0.59 & 49 & - & 1.0 to 2.9 \\
\hline Liposomes & (1.23) & $(-6)$ & - & (1.0 to 6.0 ) \\
\hline iE3/SybT & 0.99 & 15 & 44 & - \\
\hline K3/SxG & 0.77 & 33 & - & 1.0 to 3.1 \\
\hline Liposomes & (1.37) & $(-18)$ & - & (1.0 to 5.5$)$ \\
\hline E3/SybE & 0.85 & 27 & 59 & - \\
\hline K3/SxE & 0.65 & 44 & - & 1.0 to 3.0 \\
\hline Liposomes & 0.92 & 21 & - & 1.0 to 4.3 \\
\hline E3/SybK & 0.91 & 22 & 47 & - \\
\hline K3/SxK & 0.83 & 28 & - & 1.0 to 3.7 \\
\hline Liposomes & (1.33) & $(-14)$ & - & (1.0 to 5.8 ) \\
\hline E3/SybK & 1.14 & 1 & 58 & - \\
\hline K3/SxK & 0.82 & 29 & - & 1.0 to 2.9 \\
\hline Liposomes & (1.41) & $(-22)$ & - & (1.0 to 4.9 ) \\
\hline E3/SybTa & 0.82 & 29 & 65 & - \\
\hline K3/SxGa & 0.78 & 33 & - & 1.0 to 3.8 \\
\hline Liposomes & 0.98 & 15 & - & 1.0 to 4.8 \\
\hline
\end{tabular}

Remarks: a-b) Loss of lipids: a) calculated by using the supposed mass of phosphorus before extrusion $(1.16 \mu \mathrm{g})$ and measured masses of phosphorus after extrusion ( $2^{\text {nd }}$ column). b) calculated by using the measured masses of phosphorus before and after size exclusion chromatography (values are not shown). c) Actual lipid ratios between labelled ((i)E3/SybX(a)) and unlabelled (K3/SxY(a)) (proteo-)liposomes during leaflet mixing assays deviate from the supposed ratio of 1 to 4 . Abbreviations: extr.: extrusion, size ex.: size exclusion chromatography. 


\section{Determination of Proteoliposome Size}

Table A-6 Collection of sizes given as diameters of liposomes decorated with PNA-LR/TMD peptide hybrids.

\begin{tabular}{|c|c|c|c|c|c|c|}
\hline sample & size $[\mathrm{nm}]$ & average $^{a}$ & age $^{b}$ & Pdl & n(extrusions) & device $^{c}$ \\
\hline PNA1/SybT & $190 / 210$ & main size / weighted & 3 months & 0.183 & 21 & Malvern Zetasizer Nano S \\
\hline PNA1/SybE & 77 & not weighted & $3 d$ & - & 31 & DynaPro Titan \\
\hline PNA1/SybE & 87 & not weighted & $3 d$ & - & 31 & DynaPro Titan \\
\hline PNA1/SybE & $190 / 203$ & main size / weighted & 3 months & 0.172 & 21 & Malvern Zetasizer Nano S \\
\hline PNA1/SybK & 126 & not weighted & few hours & - & 31 & DynaPro Titan \\
\hline PNA1/SybEa & $164 / 180$ & main size / weighted & $20 d$ & 0.205 & 21 & Malvern Zetasizer Nano S \\
\hline PNA2/SxG & 150 & not weighted & $3 d$ & - & 31 & DynaPro Titan \\
\hline PNA3/SxG & 141 & not weighted & $3 d$ & - & 31 & DynaPro Titan \\
\hline PNA3/SxG & $190 / 209$ & main size / weighted & 3 months & 0.177 & 21 & Malvern Zetasizer Nano S \\
\hline PNA3/SxE & $164 / 169$ & main size / weighted & 4 months & 0.168 & 21 & Malvern Zetasizer Nano S \\
\hline PNA3/SxEa & 142 / 317 & main size / weighted & 2 months & 0.287 & 21 & Malvern Zetasizer Nano S \\
\hline
\end{tabular}

Remarks: By DLS measurements ascertained hydrodynamic diameters of liposomes carrying PNA-LR/TMD peptide hybrids. Liposomes with PNA1/SybX(a) peptides were labelled, those with PNA2/SxY and PNA3/SxY(a) peptides were unlabelled. Only stable proteoliposomes, i.e. proteoliposome suspensions without visible turbidity within $60 \mathrm{~min}$ after preparation, were measured: a) Three different values for the diameter are presented: "Main size" states the size of the main fraction (biggest bin) of the calculated size distribution histogram. "Weighted" and "not weighted" states the weighted and not weighted average of the calculated size distribution histogram. b) "Age" indicates the time ranges between proteoliposome preparation and DLS measurements. c) Used devices for size determination. Red lines represent the results of Section 3.4.6. 
Table A-7 Collection of sizes given as diameters of liposomes decorated with E3/K3-LR/TMD peptide hybrids.

\begin{tabular}{|c|c|c|c|c|c|c|}
\hline sample & size $[\mathrm{nm}]$ & average $^{a}$ & $\operatorname{age}^{b}$ & Pdl & aggregation $^{c}$ & method/device ${ }^{d}$ \\
\hline E3/SybT & $164 / 183$ & main size / weighted & 5 months & 0.143 & no & DLS / Malvern Zetasizer Nano S \\
\hline E3/SybT & $107 \pm 56 / 158$ & main size / not weighted & $2 d$ & & no & NTA / NanoSight \\
\hline E3/SybE & 164 / 190 & main size / weighted & 4 months & 0.147 & no & DLS / Malvern Zetasizer Nano S \\
\hline E3/SybK & 100 & not weighted & $1 d$ & & no & DLS / DynaPro Titan \\
\hline E3/SybK & $122 / 137$ & main size / weighted & $17 d$ & 0.089 & no & DLS / Malvern Zetasizer Nano S \\
\hline E3/SybK HPLC & $122 / 131$ & main size / weighted & $17 \mathrm{~d}$ & 0.133 & no & DLS / Malvern Zetasizer Nano S \\
\hline E3/SybTa & $93 \pm 64 / 145$ & main size / not weighted & few hours & & yes & NTA / NanoSight \\
\hline E3/SybKa & 126 & not weighted & few hours & & yes & DLS / DynaPro Titan \\
\hline $\mathrm{K} 3 / \mathrm{S} \times \mathrm{G}$ & $111 \pm 48 / 128$ & main size / not weighted & few hours & & yes & NTA / NanoSight \\
\hline $\mathrm{K} 3 / \mathrm{S} x \mathrm{E}$ & $142 / 158$ & main size / weighted & $17 d$ & 0.141 & no & DLS / Malvern Zetasizer Nano S \\
\hline $\mathrm{K} 3 / \mathrm{SxK}$ & $102 \pm 31 / 114$ & main size / not weighted & few hours & & yes & NTA / NanoSight \\
\hline $\mathrm{K} 3 / \mathrm{SxEa}$ & 430 & not weighted & few hours & & yes & DLS / DynaPro Titan \\
\hline $\mathrm{K} 3 / \mathrm{SxEa}$ & 1320 & not weigthed & $1 d$ & & yes & DLS / DynaPro Titan \\
\hline liposomes & 98 & not weighted & few hours & & no & DLS / DynaPro Titan \\
\hline liposomes & 109 & not weighted & $1 \mathrm{~d}$ & & no & DLS / DynaPro Titan \\
\hline liposomes & $109 \pm 50 / 167$ & main size / not weighted & $2 d$ & & no & NTA / NanoSight \\
\hline liposomes & $164 / 166$ & main size / weighted & 4 months & 0.077 & no & DLS / Malvern Zetasizer Nano S \\
\hline lab. liposomes & $122 / 141$ & main size / weighted & 4 months & 0.118 & no & DLS / Malvern Zetasizer Nano S \\
\hline
\end{tabular}

Remarks: By DLS and NTA measurements ascertained hydrodynamic diameters of liposomes carrying E3/K3-LR/TMD peptide hybrids. Liposomes with $\mathrm{E} 3 / \mathrm{SybX}(\mathrm{a})$ peptides were labelled, those with $\mathrm{K} 3 / \mathrm{S} \times \mathrm{Y}(\mathrm{a})$ were unlabelled: $\mathbf{a}-\mathbf{b}$ ) Like in table A-6. c) Aggregation was determined by visible turbidity 60 min after (proteo-)liposome preparation. d) Used devices for size determination. The E3/SybK HPLC was prepared by HPLC purified peptides. Red lines represent the results of Section 3.4.6. 


\section{7) Abbreviations}

aa

$\mathrm{Ac}_{2} \mathrm{O}$

aeg-PNA

AFM

a.u. / AU

APS

aq.

Bhoc

Boc

BLM

bp

CCD

CGMD

CMC

CMOS

CPM

Cpx

cryoTEM

DBU

DHPE

DIC

DIPEA
Amino acid

Acetic acid anhydride

Aminoethylglycine-peptide nucleic acid

Atomic force microscopy

Arbitrary unit

Ammonium persulfate

Aqueous

Benzhydryloxycarbonyl

tert-Butoxycarbonyl

Black lipid membranes

Base pair

Charge-coupled device

Coarse-grained molecular dynamics

Critcal micelle concentration

Complementary metal-oxide-semiconductor

Colloidal probe microscopy

Complexin

Cryogenic transmission electron microscopy

1,8-diazabicyclo[5.4.0]undec-7-ene

1,2-dihexadecanoyl-sn-glycero-3-phosphoethanolamine

Diisopropylcarbodiimide

Diisopropylethylamine 


\section{Abbreviations}

DLS Dynamic light scattering

DMF $\quad N, N$-Dimethylformamide

DNA Desoxyribonucleic acid

DOPC 1,2-dioleoyl-sn-glycero-3-phosphocholine

DOPE 1,2-dioleoyl-sn-glycero-3-phosphoethanolamine

DPPE 1,2-dipalmitoyl-sn-glycero-3-phosphoethanolamine

DPA Dipicolinic acid

DSC Differential scanning microscopy

DT Sodium dithionite

DTT Dithiothreitol

EDT

1,2-ethanedithiol

EDTA Ethylendiaminetetraacetate

EPR Electron paramagnetic resonance

eq.

Equivalents

ER Endoplasmic reticulum

FCS Fluorescence correlation spectroscopy

FCCS Fluorescence cross correlation spectroscopy

FFEM Freeze fracture electron microscopy

FLIC Fluorescence interference contrast

Fmoc Fluorenylmethoxycarbonyl

FP

Fusion peptide

FTIR

Fourier-transform infrared spectroscopy

FRAP

Fluorescence recovery after photobleaching

FRET

Förster resonance energy transfer 


\section{Abbreviations}

$\begin{array}{ll}\text { GUV } & \text { Giant unilamellar vesicle } \\ \text { HA } & \text { Hemagglutinin } \\ \text { HATU } & \begin{array}{l}\text { 2-(1H-9-azabenzotriazole-1-yl)-1,1,3,3-tetramethylaminium hex- } \\ \text { afluorophosphate }\end{array} \\ \text { HBTU } & \begin{array}{l}\text { 2-(1H-benzotriazole-1-yl)-1,1,3,3-tetramethylaminium hexafluoro- } \\ \text { phosphate }\end{array} \\ \text { HEPES } & \text { 4-(2-hydroxyethyl)-1-piperazineethanesulfonic acid } \\ \text { HOAt } & \text { 1-hydroxy-7-aza-1H-benzotriazole } \\ \text { HOBt } & \text { 1-hydroxybenzotriazol } \\ \text { HPLC } & \text { High performance liquid chromatography } \\ \text { ILM } & \text { Inner leaflet mixing } \\ \text { KIH } & \text { Knob-into-hole } \\ \text { LD } & \text { Loading density } \\ \text { LPC } & \text { Lysophosphatidylcholine } \\ \text { LR } & \text { Linker region } \\ \text { LSCM } & \text { Laser scanning confocal microscopy } \\ \text { LUV } & \text { Large unilamellar vesicles } \\ \text { MF } & \text { Microfluorimetry } \\ \text { MLV } & \text { Multilamellar vesicles } \\ \text { MWCO } & \text { Nolecular weight cut-off } \\ \text { NMP } & \text { Nanothyl-2,6,7-pentamethyl-dihydrobenzofurane-5-sulfonyl } \\ \text { NSF } & \text { NTA }\end{array}$




\section{Abbreviations}

PC

Phosphatidylcholine

Pdl

Polydispersity index

$\mathrm{PIP}_{2 / 3}$

Phosphatidylinositol-bis/tris-phosphate

POPC

1-palmitoyl-2-oleoyl-sn-glycero-3-phosphocholine

POPE

1-palmitoyl-2-oleoyl-sn-glycero-3-phosphoethanolamine

POPS

1-palmitoyl-2-oleoyl-sn-glycero-3-phosphocserine

PS

Phosphatidylserine

PSM

Pore spanning membrane

$\mathrm{Rh}$

Lissamine rhodamine $\mathrm{B}$

RM

Recognition motif

RNA

Ribonucleic acid

RT

Room temperature

SDS-PAGE

Sodium dodecyl sulfate-polyacrylamide gel electrophoresis

SF

Spectrofluorimetry

SNARE

Soluble N-ethylmaleimide-sensitive factor (NSF) attachment protein receptor

SM protein $\quad$ Sec and Munc protein

SPT

Single particle tracking

SUV

Small unilamellar vesicles

SNAP 25

25 kDa synaptosome-associated protein

SRB

Sulforhodamine B

SV

Synaptic vesicle

Syb2

Synaptobrevin 2

SybLR/TMD Linker region and transmembrane domain of synaptobrevin 2 


\section{Abbreviations}

Syt

Synaptotagmin

Sx1A

Syntaxin $1 \mathrm{~A}$

SxLR/TMD Linker region and transmembrane domain of syntaxin $1 \mathrm{~A}$

${ }^{t} \mathrm{Bu}$

tert-butyl

TCEP

Tris-carboxyethylphosphine

TEMED

Tetramethylethylendiamine

TFA

Trifluoroacetic acid

TFE

2,2,2-trifluoroethanol

TIRF

Total internal reflection fluorescence

TIS

Triisopropylsilane

TLM

Total leaflet mixing

TMD

Transmembrane domain

TOF

Time of flight

Tris

Tris(hydroxymethyl)aminomethane

Trt

Trityl

TxR

Texas Red

UPLC

Ultra performance liquid chromatography 


\section{8) References}

[1] E. Bianchi, B. Doe, D. Goulding, G. J. Wright, Nature 2014, 508, 483-487.

[2] J. Pérez-Vargas, T. Krey, C. Valansi, O. Avinoam, A. Haouz, M. Jamin, H. Raveh-Barak, B. Podbilewicz, F. A. Rey, Cell 2014, 157, 407-419.

[3] R. Jahn, R. H. Scheller, Nat. Rev. Mol. Cell Biol. 2006, 7, 631-643.

[4] P. Chlanda, E. Mekhedov, H. Waters, C. L. Schwartz, E. R. Fischer, R. J. Ryham, F. S. Cohen, P. S. Blank, J. Zimmerberg, Nat. Microbiol. 2016, 1, 1-8.

[5] N. A. Campbell, J. B. Reece, Biologie, Pearson Studium, München, 2006.

[6] W. Antonin, D. Fasshauer, S. Becker, R. Jahn, T. R. Schneider, Nat. Struct. Biol. 2002, 9, 107-111.

[7] K. Weninger, M. E. Bowen, S. Chu, A. T. Brunger, Proc. Natl. Acad. Sci. USA 2003, 100, 14800-14805.

[8] L. V. Chernomordik, M. M. Kozlov, Nat. Struct. Mol. Biol. 2008, 15, 675-683.

[9] A. V. Pobbati, A. Stein, D. Fasshauer Science 2006, 313, 673-676.

[10] A. Stein, G. Weber, M. C. Wahl, R. Jahn, Nature 2009, 460, 525-528.

[11] D. Langosch, M. Hofmann, C. Ungermann, Cell. Mol. Life Sci. 2007, 64, 850864.

[12] H. J. Risselada, G. Bubnis, H. Grubmuller, Proc. Natl. Acad. Sci. USA 2014, 111, 11043-11048.

[13] H. J. Risselada, H. Grubmüller, Curr. Opin. Struct. Biol. 2012, 22, 187-196.

[14] Q. Fang, M. Lindau, Physiology 2014, 29, 278-285.

[15] A. S. Lygina, K. Meyenberg, R. Jahn, U. Diederichsen, Angew. Chem. Int. Ed. 2011, 50, 8597-8601.

[16] K. Meyenberg, A. S. Lygina, G. van den Bogaart, R. Jahn, U. Diederichsen, Chem. Commun. 2011, 47, 9405.

[17] A. N. Ngatchou, K. Kislera, Q. Fang, A. M. Walter, Y. Zhao, D. Bruns, J. B. Sørensen, M. Lindau, Proc. Natl. Acad. Sci. USA. 2010, 107, 18463-18468.

[18] J.-D. Wehland, A. S. Lygina, P. Kumar, S. Guha, B. E. Hubrich, R. Jahn, U. Diederichsen, Mol. Biosyst. 2016, 12, 2770-2776.

[19] T. Weber, B. V. Zemelman, J. A. McNew, B. Westermann, M. Gmachl, F. Parlati, T. H. Söllner, J. E. Rothman, Cell 1998, 92, 759-772.

[20] J. F. Danielli, H. Davson, J. Cell. Physiol. 1935, 5, 495-508. 
[21] L. Bagatolli, P. B. Sunil Kumar, Soft Matter 2009, 5, 3234.

[22] G. L. Nicolson, Biochim. Biophys. Acta BBA - Biomembranes 2014, 1838, 14511466.

[23] J. Storch, A. M. Kleinfeld, Trends Biochem. Sci. 1985, 418-420.

[24] S. J. Singer, G. L. Nicolson, Science 1972, 175, 720-731.

[25] K. Simons, E. Ikonen, Nature 1997, 387, 569.

[26] P. R. Cullis, M. J. Hope, C. P. Tilcock, Chem. Phys. Lipids 1986, 40, 127-144.

[27] C. Tanford, Science 1978, 200, 1012-1018.

[28] R. Winter, Chem. Unserer Zeit 1990, 24, 71-81.

[29] K. M. W. Keough, P. J. Davis, Biochemistry Mosc. 1979, 18, 1453-1459.

[30] P. Y. Bruice, Organische Chemie, Pearson Studium, München, 2007.

[31] S. Takamori, M. Holt, K. Stenius, E. A. Lemke, M. Grønborg, D. Riedel, H. Urlaub, S. Schenck, B. Brügger, P. Ringler, et al., Cell 2006, 127, 831-846.

[32] F. Szoka Jr, D. Papahadjopoulos, Annu. Rev. Biophys. Bioeng. 1980, 9, 467508.

[33] H. Eibl, Angew. Chem. 1984, 96, 247-262.

[34] J. N. Israelachvili, S. Marcelja, R. G. Horn, Q. Rev. Biophys. 1980, 13, 121.

[35] L. J. Foster, C. L. de Hoog, M. Mann, Proc. Natl. Acad. Sci. USA 2003, 100, 5813-5818.

[36] C. Dietrich, L. A. Bagatolli, Zn. Volovyk, N. L. Thompson, M. Levi, K. Jacobson, E. Gratton, Biophys. J. 2001, 80, 1417-1428.

[37] D. Milovanovic, R. Jahn, Front. Physiol. 2015, 6, 1-11, DOI 10.3389/fphys.2015.00089.

[38] N. Kucerka, M. Nieh, J. Pencer, J. Sachs, J. Katsaras, Gen. Physiol. Biophys. 2009, 28, 117-125.

[39] R. Hine, The Facts on File Dictionary of Biology., Checkmark Books, New York, 1999, p. 198.

[40] L. V. Chernomordik, M. M. Kozlov, Annu. Rev. Biochem. 2003, 72, 175-207.

[41] G. E. Palade, R. R. Bruns, J. Cell Biol. 1968, 37, 633-649.

[42] M. M. Kozlov, V. S. Markin, Biofizika 1983, 28, 242-247.

[43] L. Yang, Science 2002, 297, 1877-1879.

[44] Y. Kozlovsky, M. M. Kozlov, Biophys. J. 2002, 82, 882-895. 
[45] S. Aeffner, T. Reusch, B. Weinhausen, T. Salditt, Proc. Natl. Acad. Sci. USA 2012, 109, E1609-E1618.

[46] R. P. Rand, V. A. Parsegian, BBA - Rev. Biomembranes 1989, 988, 351-376.

[47] Y. G. Smirnova, S.-J. Marrink, R. Lipowsky, V. Knecht, J. Am. Chem. Soc. 2010, 132, 6710-6718.

[48] A. Efrat, L. V. Chernomordik, M. M. Kozlov, Biophys. J. 2007, 92, L61-L63.

[49] P. I. Kuzmin, J. Zimmerberg, Y. A. Chizmadzhev, F. S. Cohen, Proc. Natl. Acad. Sci. USA 2001, 98, 7235-7240.

[50] S. A. Akimov, R. J. Molotkovsky, T. R. Galimzyanov, A. V. Radaev, L. A. Shilova, P. I. Kuzmin, O. V. Batishchev, G. F. Voronina, Y. A. Chizmadzhev, Biochem. (Mosc.) Suppl. Ser. A Membr. Cell Biol. 2014, 8, 153-161.

[51] A. Chanturiya, L. V. Chernomordik, J. Zimmerberg, Proc. Natl. Acad. Sci. USA 1997, 94, 14423-14428.

[52] B. S. Stratton, J. M. Warner, Z. Wu, J. Nikolaus, G. Wei, E. Wagnon, D. Baddeley, E. Karatekin, B. O'Shaughnessy, Biophys. J. 2016, 110, 1538-1550.

[53] S. Günther-Ausborn, T. Stegmann, Virology 1997, 235, 201-208.

[54] L. Chernomordik, A. Chanturiya, J. Green, J. Zimmerberg, Biophys. J. 1995, 69, 922-929.

[55] Z. Chen, R. P. Rand, Biophys. J. 1997, 73, 267-276.

[56] H. R. Marsden, I. Tomatsu, A. Kros, Chem Soc Rev 2011, 40, 1572-1585.

[57] X. Chen, D. Araç, T.-M. Wang, C. J. Gilpin, J. Zimmerberg, J. Rizo, Biophys. J. 2006, 90, 2062-2074.

[58] D. Milovanovic, M. Platen, M. Junius, U. Diederichsen, I. A. T. Schaap, A. Honigmann, R. Jahn, G. van den Bogaart, J. Biol. Chem. 2016, 291, 7868-7876.

[59] T. Südhof, Neuron 2013, 80, 675-690.

[60] J. A. McNew, T. Weber, F. Parlati, R. J. Johnston, T. J. Melia, T. H. Söllner, J. E. Rothman, J. Cell Biol. 2000, 150, 105-118.

[61] C.-W. Chang, M. Jackson, Biophys. J. 2015, 109, 76-84.

[62] A. Anantharam, M. A. Bittner, R. L. Aikman, E. L. Stuenkel, S. L. Schmid, D. Axelrod, R. W. Holz, Mol. Biol. Cell 2011, 22, 1907-1918.

[63] M. M. Kozlov, L. V. Chernomordik, Curr. Opin. Struct. Biol. 2015, 33, 61-67.

[64] A. L. Lai, H. Park, J. M. White, L. K. Tamm, J. Biol. Chem. 2006, 281, 57605770 . 
[65] T. Stegmann, Traffic 2000, 1, 598-604.

[66] B. Podbilewicz, Annu. Rev. Cell Dev. Biol. 2014, 30, 111-139.

[67] H. J. Risselada, C. Kutzner, H. Grubmüller, ChemBioChem 2011, 12, 10491055.

[68] X. Peng, J. 'an Pan, R. Gong, Y. Liu, S. Kang, H. Feng, G. Qiu, D. Guo, P. Tien, G. Xiao, J. Biol. Chem. 2007, 282, 381-389.

[69] G. Orso, D. Pendin, S. Liu, J. Tosetto, T. J. Moss, J. E. Faust, M. Micaroni, A. Egorova, A. Martinuzzi, J. A. McNew, et al., Nature 2009, 460, 978-983.

[70] J. Hu, T. A. Rapoport, Semin. Cell Dev. Biol. 2016, 60, 105-111.

[71] S. Hoppins, J. Nunnari, BBA - Mol. Cell Res. 2009, 1793, 20-26.

[72] A. M. van der Bliek, Q. Shen, S. Kawajiri, Cold Spring Harb. Perspect. Biol. 2013, 5, 1-16.

[73] T. Koshiba, Science 2004, 305, 858-862.

[74] Y.-L. Cao, S. Meng, Y. Chen, J.-X. Feng, D.-D. Gu, B. Yu, Y.-J. Li, J.-Y. Yang, S. Liao, D. C. Chan, et al., Nature 2017, 542, 372-376.

[75] F. Li, D. Kümmel, J. Coleman, K. M. Reinisch, J. E. Rothman, F. Pincet, J. Am. Chem. Soc. 2014, 136, 3456-3464.

[76] Y. Gao, S. Zorman, G. Gundersen, Z. Xi, L. Ma, G. Sirinakis, J. E. Rothman, Y. Zhang, Science 2012, 337, 1340-1343.

[77] V. Kiessling, B. Liang, A. J. B. Kreutzberger, L. K. Tamm, Front. Mol. Neurosci. 2017, 10, 1-8, DOI 10.3389/fnmol.2017.00072.

[78] N. R. Gandasi, S. Barg, Nat. Commun. 2014, 5, 1-14 DOI 10.1038/ncomms4914.

[79] C. Ma, L. Su, A. B. Seven, Y. Xu, J. Rizo, Science 2013, 339, 421-425.

[80] K. Weninger, M. E. Bowen, U. B. Choi, S. Chu, A. T. Brunger, Structure 2008, 16, 308-320.

[81] M. Xue, K. Reim, X. Chen, H.-T. Chao, H. Deng, J. Rizo, N. Brose, C. Rosenmund, Nat. Struct. Mol. Biol. 2007, 14, 949-958.

[82] E. Hui, C. P. Johnson, J. Yao, F. M. Dunning, E. R. Chapman, Cell 2009, 138, 709-721.

[83] A. Honigmann, G. van den Bogaart, E. Iraheta, H. J. Risselada, D. Milovanovic, V. Mueller, S. Müllar, U. Diederichsen, D. Fasshauer, H. Grubmüller, et al., Nat. Struct. Mol. Biol. 2013, 20, 679-686. 
[84] T. A. M. Bharat, J. Malsam, W. J. H. Hagen, A. Scheutzow, T. H. Söllner, J. A. G. Briggs, EMBO Rep. 2014, 15, 308-314.

[85] S. Sharma, M. Lindau, Nat. Struct. Mol. Biol. 2016, 23, 5-6.

[86] Y.-H. M. Chan, S. G. Boxer, Curr. Opin. Chem. Biol. 2007, 11, 581-587.

[87] D. Papahadjopoulos, S. Hui, W. Vail, G. Poste, BBA - Biomembranes 1976, 448, 245-264.

[88] L. L. G. Schwenen, R. Hubrich, D. Milovanovic, B. Geil, J. Yang, A. Kros, R. Jahn, C. Steinem, Sci. Rep. 2015, 5, 1-15, DOI 10.1038/srep12006.

[89] D. Papahadjopoulos, A. D. Bangham, Biochim. Biophys. Acta 1966, 126, 185188.

[90] V. Torchilin, V. Weissig, Liposomes - A Practical Approach, Oxford University Press, Oxford, 2003.

[91] Y. Barenholzt, S. Amselem, D. Lichtenberg, FEBS Lett. 1979, 99, 210-214.

[92] M. J. Hope, M. B. Bally, G. Webb, P. R. Cullis, BBA - Biomembranes 1985, 812, 55-65.

[93] R. C. MacDonald, R. I. MacDonald, B. P. M. Menco, K. Takeshita, N. K. Subbarao, L. Hu, BBA - Biomembranes 1991, 1061, 297-303.

[94] M. I. Angelova, S. Soléau, P. Méléard, F. Faucon, P. Bothorel, Progr. Colloid Polym. Sci. 1992, 89, 127-131.

[95] C. Ropert, Braz. J. Med. Biol. Res. 1999, 32, 163-169.

[96] P. Mueller, D. O. Rudin, H. T. Tien, W. C. Wescott, J. Phys. Chem. 1963, 67, 534-535.

[97] M. Winterhalter, Curr. Opin. Colloid Interface Sci. 2000, 5, 250-255.

[98] E. Sackmann, Science. 1996, 271, 43-48.

[99] E. Kalb, S. Frey, L. K. Tamm, BBA - Biomembranes 1992, 1103, 307-316.

[100] J. W. Kuhlmann, M. Junius, U. Diederichsen, C. Steinem, Biophys. J. 2017, 112, 2348-2356.

[101] D. Papahadjopoulos, W. J. Vail, C. Newton, S. Nir, K. Jacobson, G. Poste, R. Lazo, BBA - Biomembranes 1977, 465, 579-598.

[102] A. Portis, C. Newton, W. Pangborn, D. Papahadjopoulos, Biochemistry Mosc. 1979, 18, 780-790.

[103] D. Papahadjopoulos, S. Nir, N. Düzgünes, J. Bioenerg. Biomembr. 1990, 22, 157-179. 
[104] Y.-H. M. Chan, B. van Lengerich, S. G. Boxer, Biointerphases 2008, 3, FA17FA21.

[105] Y.-H. M. Chan, B. van Lengerich, S. G. Boxer, Proc. Natl. Acad. Sci. USA 2009, 106, 979-984.

[106] G. Stengel, R. Zahn, F. Höök, J. Am. Chem. Soc. 2007, 129, 9584-9585.

[107] G. Stengel, L. Simonsson, R. A. Campbell, F. Höök, J. Phys. Chem. B 2008, 112, 8264-8274.

[108] H. Robson Marsden, N. A. Elbers, P. H. H. Bomans, N. A. J. M. Sommerdijk, A. Kros, Angew. Chem. 2009, 121, 2366-2369.

[109] M. Ma, Y. Gong, D. Bong, J. Am. Chem. Soc. 2009, 131, 16919-16926.

[110] G. N. Tew, D. Liu, B. Chen, R. J. Doerksen, J. Kaplan, P. J. Carroll, M. L. Klein, W. F. DeGrado, Proc. Natl. Acad. Sci. USA 2002, 99, 5110-5114.

[111] C.-W. Chang, M. B. Jackson, Biophys. J. 2015, 109, 76-84.

[112] P. E. Nielsen, M. Egholm, R. H. Berg, O. Buchardt, Science 1991, 1497-1500.

[113] H. Rasmussen, J. Sandholm Kastrup, J. Nederby Nielsen, J. M. Nielsen, P. E. Nielsen, Nat. Struct. Biol. 1997, 4, 98-101.

[114] P. Wittung, P. E. Nielsen, O. Buchardt, M. Egholm, B. Norden, Nature 1994, 368, 561-563.

[115] S. Tomac, M. Sarkar, T. Ratilainen, P. Wittung, P. E. Nielsen, B. Nordén, A. Gräslund, J. Am. Chem. Soc. 1996, 118, 5544-5552.

[116] B. Hyrup, P. E. Nielsen, Bioorg. Med. Chem. 1996, 4, 5-23.

[117] M. E. Bowen, K. Weninger, A. T. Brunger, S. Chu, Biophys. J. 2004, 87, 35693584.

[118] R. B. Sutton, D. Fasshauer, R. Jahn, A. T. Brunger, Nature 1998, 395, 347-353.

[119] G. N. Phillips, Proteins - Structure Function and Genetics. 1992, 14, 425-429.

[120] D. A. Lindhout, J. R. Litowski, P. Mercier, R. S. Hodges, B. D. Sykes, Biopolymers 2004, 75, 367-375.

[121] J. Walshaw, D. N. Woolfson, J. Mol. Biol. 2001, 307, 1427-1450.

[122] D. N. Woolfson, T. Alber, Protein Sci. 1995, 4, 1596-1607.

[123] P. B. Harbury, T. Zhang, P. S. Kim, T. Alber, Science 1993, 262, 1401-1401.

[124] D. N. Woolfson, Adv. Protein Chem. 2005, 70, 79-112.

[125] D. N. Woolfson, Fibrous Proteins: Structures and Mechanisms, Springer International Publishing, Cham, 2017, pp. 35-61. 
[126] J. R. Litowski, R. S. Hodges, J. Biol. Chem. 2002, 277, 37272-37279.

[127] J. A. Ernst, A. T. Brunger, J. Biol. Chem. 2003, 278, 8630-8636.

[128] E. J. Shimshick, H. M. McConnell, Biochemistry Mosc. 1973, 12, 2351-2360.

[129] K. Halbmair, J. Wegner, U. Diederichsen, M. Bennati, Biophys. J. 2016, 111, 2345-2348.

[130] M. Fröhlich, V. Brecht, R. Peschka-Süss, Chem. Phys. Lipids 2001, 109, 103112.

[131] M. G. Taylor, T. Akiyama, I. C. Smith, Chem. Phys. Lipids 1981, 29, 327-339.

[132] M. Caffrey, J. Wang, Annu. Rev. Biophys. Biomol. Struct. 1995, 24, 351-377.

[133] M. C. Phillips, B. D. Ladbrooke, D. Chapman, Biochim. Biophys. Acta 1970, 196, 35-44.

[134] R. N. A. H. Lewis, R. N. McElhaney, BBA - Biomembranes 2013, 1828, 23472358.

[135] N. J. Severs, Nat. Protoc. 2007, 2, 547-576.

[136] L. Issman, B. Brenner, Y. Talmon, A. Aharon, PLoS ONE 2013, 8, 1-9.

[137] S. W. Paddock, Confocal Microscopy - Methods and Protocols, Humana Press, Totowa, 1999, pp. v-vi.

[138] D. Axelrod, Methods in Cell Biology 2008, 89, 169-221.

[139] J. Yguerabide, J. A. Schmidt, E. E. Yguerabide, Biophys. J. 1982, 39, 69-75.

[140] E. Thews, M. Gerken, R. Eckert, J. Zäpfel, C. Tietz, J. Wrachtrup, Biophys. J. 2005, 89, 2069-2076.

[141] J. Sankaran, M. Manna, L. Guo, R. Kraut, T. Wohland, Biophys. J. 2009, 97, 2630-2639.

[142] A. Cypionka, A. Stein, J. M. Hernandez, H. Hippchen, R. Jahn, P. J. Walla, Proc. Natl. Acad. Sci. USA 2009, 106, 18575-18580.

[143] G. P. Gorbenko, J. Fluoresc. 2011, 21, 945-951.

[144] S. Deshayes, G. Divita, in Prog. Mol. Biol. Transl. Sci. 2013, 113, 109-143.

[145] T. Förster, Ann. Phys. 1948, 437, 55-75.

[146] S. A. Hussain, D. Dey, S. Chakraborty, J. Saha, A. D. Roy, S. Chakraborty, P. Debnath, D. Bhattacharjee, Fluorescence Resonance Energy Transfer (FRET) Sensor, Cornell University Library, New York, 2014.

[147] L. Loura, Front. Physiol. 2011, 2, 1-11, DOI 10.3389/fphys.2011.00082. 
[148] D. K. Struck, D. Hoekstra, R. E. Pagano, Biochemistry Mosc. 1981, 20, 40934099.

[149] J. C. McIntyre, R. G. Sleight, Biochemistry Mosc. 1991, 30, 11819-11827.

[150] P. Meers, S. Ali, R. Erukulla, A. S. Janhoff, Biochim. Biophys. Acta 2000, 1467, 227-243.

[151] G. van den Bogaart, M. G. Holt, G. Bunt, D. Riedel, F. S. Wouters, R. Jahn, Nat. Struct. Mol. Biol. 2010, 17, 358-364.

[152] M. Kyoung, A. Srivastava, Y. Zhang, J. Diao, M. Vrljic, P. Grob, E. Nogales, S. Chu, A. T. Brunger, Proc. Natl. Acad. Sci. USA 2011, 108, E304-E313.

[153] T. Wang, E. A. Smith, E. R. Chapman, J. C. Weisshaar, Biophys. J. 2009, 96, 4122-4131.

[154] J. Wilschut, N. Duzgunes, R. Fraley, D. Papahadjopoulos, Biochemistry Mosc. 1980, 19, 6011-6021.

[155] J. Otterstrom, A. M. van Oijen, Biochemistry Mosc. 2013, 52, 1654-1668.

[156] B. Lorenz, R. Keller, E. Sunnick, B. Geil, A. Janshoff, Biophys. Chem. 2010, 150, 54-63.

[157] R. B. Merrifield, J. Am. Chem. Soc. 1963, 85, 2149-2154.

[158] E. Atherton, H. Fox, D. Harkiss, C. J. Logan, R. C. Sheppard, B. J. Williams, J. Chem. Soc. Chem. Commun. 1978, 537-539.

[159] W. C. Chan, P. D. White, Fmoc Solid Phase Peptide Synthesis: A Practical Approach, Oxford University Press, New York, 2000.

[160] Bachem AG, Solid Phase Peptide Synthesis Bachem - Pioneering Partner for Peptides, Global Marketing Bachem Group, 2016.

[161] G. Pähler, C. Panse, U. Diederichsen, A. Janshoff, Biophys. J. 2012, 103, $2295-$ 2303.

[162] G. R. Bartlett, J. Biol. Chem. 1959, 234, 466-468.

[163] W. D. Harris, P. Popat, J. Am. Oil Chem. Soc. 1954, 31, 124-127.

[164] T. Zheng, M. Bulacu, G. Daudey, F. Versluis, J. Voskuhl, G. Martelli, J. Raap, G. J. A. Sevink, A. Kros, A. L. Boyle, RSC Adv 2016, 6, 7990-7998.

[165] M. Sadek, D. Berndt, D. Milovanovic, R. Jahn, U. Diederichsen, ChemBioChem 2016, 17, 479-485.

[166] S. Y. Woo, H. Lee, Sci. Rep. 2016, 6, 1-13, DOI 10.1038/srep22299. 
[167] M. Rabe, C. Aisenbrey, K. Pluhackova, V. de Wert, A. L. Boyle, D. F. Bruggeman, S. A. Kirsch, R. A. Böckmann, A. Kros, J. Raap, et al., Biophys. J. 2016, 111, 2162-2175.

[168] D. P. Tieleman, S.-J. Marrink, J. Am. Chem. Soc. 2006, 128, 12462-12467.

[169] M. Langer, D. Langosch, FEBS Lett. 2011, 585, 1021-1024.

[170] A. Lygina, Design, Synthesis and Fusion Activity of PNA/Peptide Hybrids as SNARE Protein Models, Doctoral thesis, Georg-August-University Göttingen, 2011.

[171] S. Özdirekcan, T. K. M. Nyholm, M. Raja, D. T. S. Rijkers, R. M. J. Liskamp, J. A. Killian, Biophys. J. 2008, 94, 1315-1325.

[172] Y. Fujiki, A. L. Hubbard, S. Fowler, P. B. Lazarow, J. Cell Biol. 1982, 93, 97102.

[173] S. Fery-Forgues, J.-P. Fayet, A. Lopez, J. Photochem. Photobiol. A Chem. 1993, 70, 229-243.

[174] N. Schmidt, Coiled Coil-bildende Peptide als Sensor zur Quantifizierung von T ransmembran-Peptid-Insertion und als auslösendes Element von Membranfusion, Bachelor thesis, Georg-August-University Göttingen, 2016.

[175] C. S. Chong, K. Colbow, BBA - Biomembranes 1976, 436, 260-282.

[176] Malvern Instruments Ltd., ZetasizerNano Series - User Manual 2013.

[177] Wyatt Technology Corporation, DynaPro NanoStar - User's Guide 2009.

[178] K. Meyenberg, Synthese und Untersuchungen von peptidischen Modellsystemen für SNARE-induzierte Membranfusion, Doctoral thesis, Georg-August-University Göttingen, 2011.

[179] NanoSight Ltd., NanoSight - NTA 2.3 Analytical Software 2012.

[180] M. Oelkers, H. Witt, P. Halder, R. Jahn, A. Janshoff, Proc. Natl. Acad. Sci. USA 2016, 113, 13051-13056.

[181] H. Witt, F. Savić, M. Oelkers, S. I. Awan, D. B. Werz, B. Geil, A. Janshoff, Biophys. J. 2016, 110, 1582-1592.

[182] W. C. Wimley, S. H. White, Nat. Struct. Biol. 1996, 3, 842-848.

[183] F. W. S. Stetter, L. Cwiklik, P. Jungwirth, T. Hugel, Biophys. J. 2014, 107, 11671175.

[184] D. N. Ganchev, D. T. S. Rijkers, M. M. E. Snel, J. A. Killian, B. de Kruijff, Biochemistry Mosc. 2004, 43, 14987-14993. 
[185] H. Häkkinen, Nat. Chem. 2012, 4, 443-455.

[186] G. Bell, Science 1978, 200, 618-627.

[187] E. Evans, K. Ritchie, Biophys. J. 1997, 72, 1541-1555.

[188] M. Rabe, A. Boyle, H. R. Zope, F. Versluis, A. Kros, Biopolymers 2015, 104, 65-72.

[189] M. Gude, J. Ryf, P. D. White, Lett. Pept. Sci. 2002, 9, 203-206.

[190] P. Liu, B. W. O'Mara, B. M. Warrack, W. Wu, Y. Huang, Y. Zhang, R. Zhao, M. Lin, M. S. Ackerman, P. K. Hocknell, et al., J. Am. Soc. Mass Spectrom. 2010, 21, 837-844.

[191] T. H. Fischer, D. D. Lasic, Mol. Cryst. Liq. Cryst. 1984, 102, 141-153.

[192] T. M. Allen, A. Y. Romans, H. Kercret, J. P. Segrest, Biochim. Biophys. Acta BBA - Biomembranes 1980, 601, 328-342.

[193] C. Steinem, Praktikum Biomolekulare Chemie im Masterstudiengang Chemie, Georg-August-University Göttingen, 2013.

[194] P. E. Nielsen, Peptide Nucleic Acids - Protocols and Applications, Horizon Bioscience, Norfolk, 2004.

[195] H. Edelhoch, Biochemistry Mosc. 1967, 6, 1948-1954.

[196] R. Subirós-Funosas, S. N. Khattab, L. Nieto-Rodriguez, A. El-Faham, F. Albericio, Aldrichimica Acta 2013, 46, 21-40.

[197] E. Valeur, M. Bradley, Chem Soc Rev 2009, 38, 606-631.

[198] D. A. Pearson, M. Blanchette, M. L. Baker, C. A. Guindon, Tetrahedron Lett. 1989, 30, 2739-2742.

[199] B. F. Lundt, N. L. Johansen, A. Vølund, J. Markussen, Chem. Biol. Drug Des. 1978, 12, 258-268.

[200] J. L. Lauer, C. G. Fields, G. B. Fields, Lett. Pept. Sci. 1995, 1, 197-205.

[201] Y. Han, F. Albericio, G. Barany, J. Org. Chem. 1997, 62, 4307-4312. 


\section{Acknowledgements}

First of all, I want to thank Prof. Dr. Ulf Diederichsen to give me the chance to work in his group for my doctoral thesis. As a "Lehramtsstudent" it was not natural for me to have this chance. Despite a difficult start of the dissertation, Mr. Diederichsen grant me time to develop personally and scientifically. It was a great time and I gain much experience for arising tasks.

I thank Prof. Dr. Reinhard Jahn to take over the position of the second reviewer and to give me the possibility to learn new methods concerning membranes in his laboratory.

I am grateful for the support of Antonina Lygina and Karsten Meyenberg who share their experience in the field of membrane peptides with me.

I thank all former and present members of the "AK Diederichsen" for the support during my daily work. I had much helpful discussions and without them, it was not possible to finish my thesis. Especially the members of lab 102 I give thanks for the nice concentrated atmosphere, help in practical issues and nice discussions: Brigitte Worbs with her great experience and Cornelia Panse who help me to settle in lab 102. Moreover, Julia Graf who starts her doctoral thesis at the same time as me and Dina Zanbot who works in the same lab for many years. Last but not least, I thank my "desk neighbour" Barbara Hubrich because I cannot imagine a colleague who is more friendly and helpful.

Sven Bölke, Pirajeev Selvachandran and Nina Schmidt support my work during their diploma and bachelor theses. I am very thankful for their motivated effort in the lab.

I thank Samit Guha, Pawan Kumar and Matthias Körling for their support during their postdoc time in our group. Special thanks is to Matthias who help much to achieve progress concerning the purity of our peptides.

I am grateful for Aoife Neville and Angela Heinemann who always help me concerning issues of organisation and administration.

Furthermore, I thanks the AK Thomas for their support and helpful discussions. 


\section{$\underline{\text { Acknowledgements }}$}

Hannes Witt and Marian Vache of the AK Janshoff as well as Raphael Hubrich of the AK Steinem I thank for the performance of the atomic force microscopy and the pore spanning membrane measurements.

My family I want to thank for the time of relaxing in my home village Barnstedt. All my friends I thank for the great times during vacation in the "Bulli" or during short trips to amazing cities all over Europe. Special thanks is to Angela Hinkel who share her life with me despite my long days in the lab and who always motivated me to proceed. 


\section{Curriculum Vitae}

\section{Curriculum Vitae}

Surname, Name

Date and place of birth

Citizenship

\section{Education}

08/2012-11/2017

05/2011-05/2012

$10 / 2009-05 / 2012$

04/2009-07/2009

$10 / 2006-07 / 2009$

1999-2006
Wehland, Jan-Dirk

$31^{\text {st }}$ July 1986 , in Verden/Aller

Federal Republic of Germany
Doctoral thesis in the group of Prof. Dr. Ulf Diederichsen, Institute of Organic and Biomolecular Chemistry, Georg-August University Göttingen

Title: "Hybrids of SNARE Transmembrane Domains and Aritificial Recognition Motifs as Membrane Fusion Inducing Model Peptides"

Master thesis in the group of Prof. Dr. Ulf Diederichsen

Title: "Transmembranpeptide mit Peptidnucleinsaure-Erkennungseinheit: Synthese und Untersuchung im Hinblick auf die Induktion von Membranfusion“

Master of Education at the Georg-August University Göttingen (subjects: chemistry and biology)

Bachelor thesis in the group of Prof. Dr. Ulf Diederichsen Title: "Synthese deuterierter Fettsäuren für mechanistische Studien am Enzym Psi-factor-producing-oxygenase $(P p o A)^{\star \prime}$

Bachelor of Arts at the Georg-August University of Göttingen (subjects: chemistry and biology)

Allgemeine Hochschulreife at the Domgymnasium Verden/Aller 


\section{Teaching Experience}

04/2013-07/2013

04/2014-07/2014

06/2014-07/2014

$10 / 2015$

07/2015

07/2016
Teaching assistant in the course "Praktikum Experimentalchemie II" for biochemists (B.Sc.)

Teaching assistant in the course "Tutorium Experimentalchemie II" for chemists (B.Sc.)

Teaching assistant in the course "Chemisches Praktikum für Studierende der Human- und Zahnmedizin" Teaching assistant in the course "Praktikum Biologische Chemie" for biochemists (B.Sc.)

Teaching assistant in the course "Biological Chemistry - Practical Course" for biochemists (M.Sc.)

Teaching assistant in the course "Biological Chemistry - Practical Course" for biochemists (M.Sc.)

\section{Publications}

J.-D. Wehland, A. S. Lygina, P. Kumar, S. Guha, B. E. Hubrich, R. Jahn, U. Diederichsen, Mol. Biosyst. 2016, 12, 2770-2776.

A. Nadler, C. Koch, F. Brodhun, J.-D. Wehland, K. Tittmann, I. Feussner, U. Diederichsen, ChemBioChem 2011, 12, 728-737. 